\title{
Small extracellular vesicles convey the stress response of tumour cells
}

\author{
PhD Thesis
}

Mária Harmati

\section{Supervisor:}

Krisztina Buzás, $\mathrm{PhD}$

Faculty of Dentistry, University of Szeged

Biological Research Centre
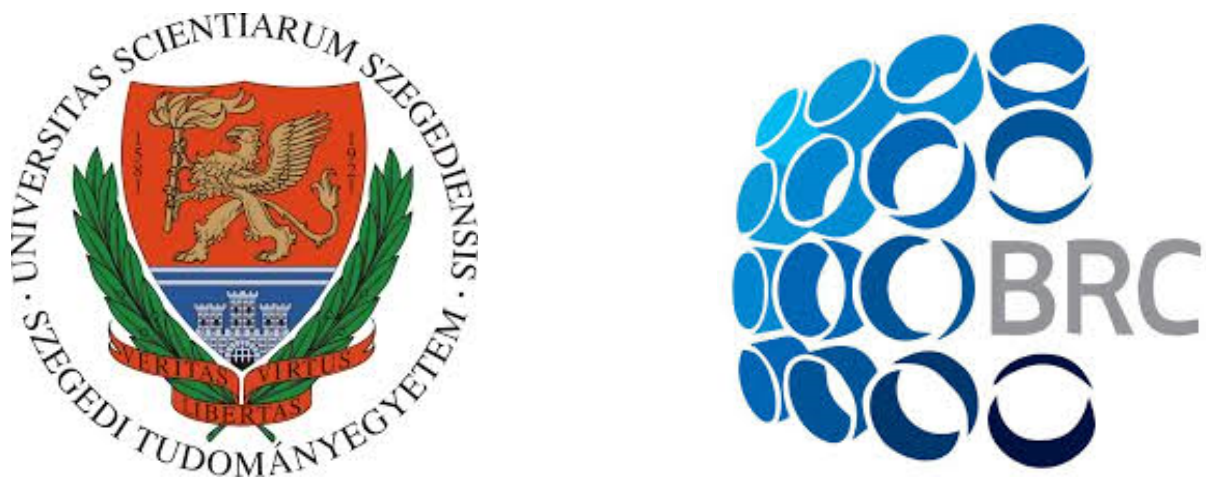

Doctoral School of Interdisciplinary Medicine,

Faculty of Medicine, University of Szeged

Szeged 
The dissertation is based on the following publications:

I. Harmati M, Tarnai Z, Decsi G, Kormondi S, Szegletes Z, Janovak L, Dekany I, Saydam O, Gyukity-Sebestyen E, Dobra G, Nagy I, Nagy K, Buzas K. Stressors alter intercellular communication and exosome profile of nasopharyngeal carcinoma cells. $J$ Oral Pathol Med. 46, 259-266, DOI: https://doi.org/10.1111/jop.12486 (2017).

IF: 2.237; Q2

II. Harmati M, Gyukity-Sebestyen E, Dobra G, Janovak L, Dekany I, Saydam O, Hunyadi-Gulyas E, Nagy I, Farkas A, Pankotai T, Ujfaludi Zs, Horvath P, Piccinini F, Kovacs M, Biro T, Buzas K. Small extracellular vesicles convey the stress-induced adaptive responses of melanoma cells. Sci Rep. 9, 15329; DOI: https://doi.org/10.1038/s41598-019-51778-6 (2019).

Estimated IF: 4.011; Q1

Other publications related to the subject of the thesis:

III. Gyukity-Sebestyen E, Harmati M, Dobra G, Nemeth I B, Mihaly J, Zvara Á, HunyadiGulyas E, Katona R, Nagy I, Horvath P, Balind A, Szkalisity A, Kovacs M, Pankotai T, Borsos B, Erdelyi M, Szegletes Zs, Vereb Z, Buzas E I, Kemeny L, Biro T, Buzas K. Melanoma-Derived Exosomes Induce PD-1 Overexpression and Tumor Progression via Mesenchymal Stem Cell Oncogenic Reprogramming. Front. Immunol. 10, 2459; DOI: https://doi.org/10.3389/fimmu.2019.02459 (2019).

Estimated IF: 4.716; Q1

Other full papers published during the $\mathrm{PhD}$ fellowship:

IV. Buzas K, Marton A, Vizler C, Gyukity-Sebestyen E, Harmati M, Nagy K, Zvara A, Katona RL, Tubak V, Endresz V, Nemeth IB, Olah J, Vigh L, Biro T, Kemeny L. Bacterial sepsis increases survival in metastatic melanoma: Chlamydophila pneumoniae induces macrophage polarization and tumor regression. J. Invest. Dermatol. 136, 862-865. DOI: https://doi.org/10.1016/j.jid.2015.12.032 (2016) 
V. Harmati M, Gyukity-Sebestyen E, Dobra G, Terhes G, Urban E, Decsi G, MimicaDukic N, Lesjak M, Simin N, Pap B, Nemeth IB, Buzas K. Binary mixture of Satureja hortensis and Origanum vulgare subsp. hirtum essential oils: in vivo therapeutic efficiency against Helicobacter pylori infection. Helicobacter. 22, e12350; DOI: https://doi.org/10.1111/hel.12350 (2017).

IF 4.123; Q1

VI. Zsedenyi A, Farkas B, Abdelrasoul GN, Romano I, Gyukity-Sebestyen E, Nagy K, Harmati M, Dobra G, Kormondi S, Decsi G, Nemeth IB, Diaspro A, Brandi F, Beke S, Buzas K. Gold nanoparticle-filled biodegradable photopolymer scaffolds induced muscle remodeling: in vitro and in vivo findings. Mater Sci Eng C Mater Biol Appl. 72, 625-630. DOI: https://doi.org/10.1016/j.msec.2016.11.124 (2017)

IF 5.080; Q1

VII. Decsi G, Soki J, Pap B, Dobra G, Harmati M, Kormondi S, Pankotai T, Braunitzer G, Minarovits J, Sonkodi I, Urban E, Nemeth IB, Nagy K, Buzas K. Chicken or the Egg: Microbial Alterations in Biopsy Samples of Patients with Oral Potentially Malignant Disorders. Pathol Oncol Res. 25, 1023-1033. DOI: https://doi.org/10.1007/s12253018-0457-x (2019).

Estimated IF 2.433; Q2

VIII. Körmöndi S, Terhes G, Pal Z, Varga E, Harmati M, Buzas K, Urban E. Human Pasteurellosis Health Risk for Elderly Persons Living with Companion Animals. Emerg Infect Dis. 25, 229-235. DOI: https://doi.org/10.3201/eid2502.180641 (2019).

Estimated IF 7.185; Q1

Cumulative IF 36.072 


\section{Table of contents}

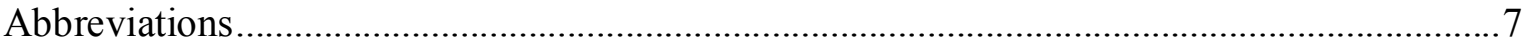

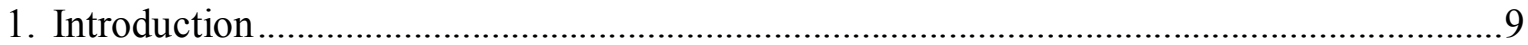

1.1 Exosomes, the endosome-derived small extracellular vesicles ...............................

1.2 Exosomal communication in the tumour microenvironment ................................10

$1.3 \quad$ Role of exosomes in nasopharyngeal carcinoma..................................................12

1.4 Role of exosomes in malignant melanoma ............................................................13

1.5 Role of intrinsic microenvironmental factors in the exosome-mediated

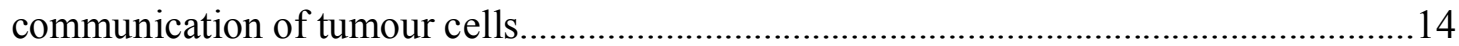

1.6 Effects of different therapeutic conditions on the exosome-mediated

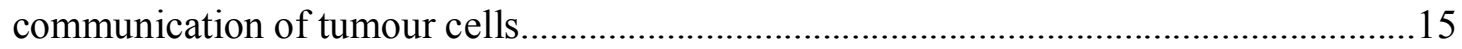

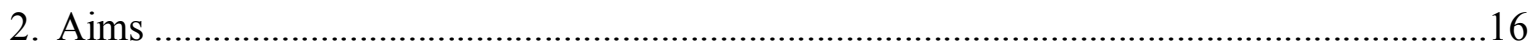

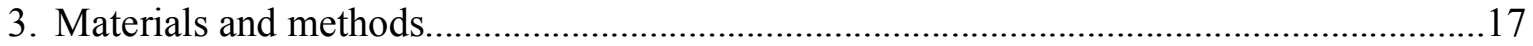

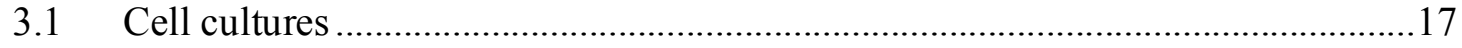

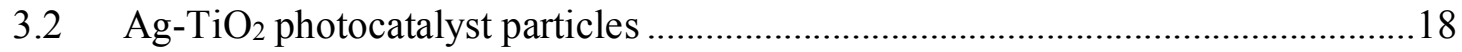

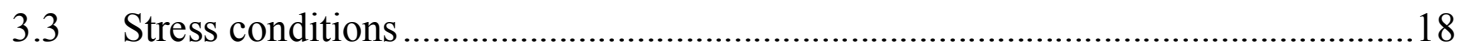

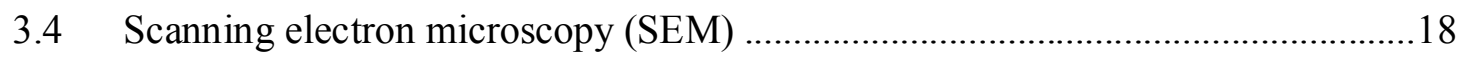

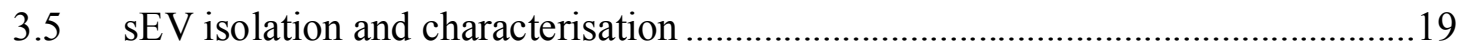

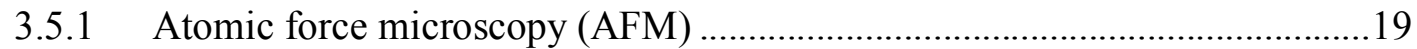

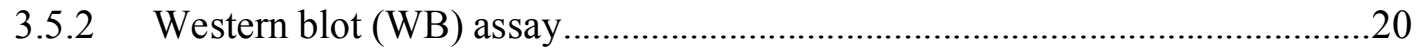

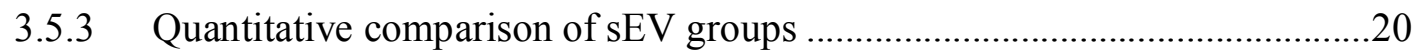

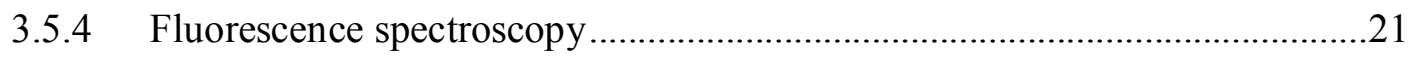

3.5.5 Dynamic light scattering (DLS) measurements of $\mathrm{Ag}-\mathrm{TiO}_{2}$ particles............21

3.5.6 Chemiluminescence (CL) detection of $\mathrm{Ag}-\mathrm{TiO}_{2}$ nanoparticles ......................21

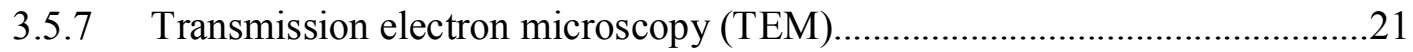

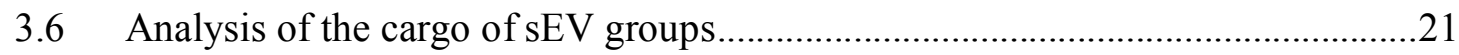




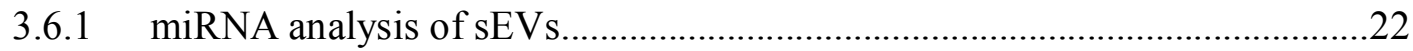

3.6.2 Protein analysis of B16F1 -derived sEVs......................................................23

3.7 Functional comparison of the sEV groups ...........................................................23

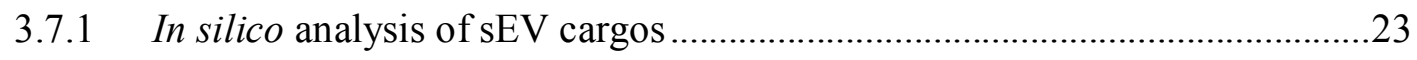

3.7.2 In vitro assays for functional comparison of the B16F1 sEV groups.............24

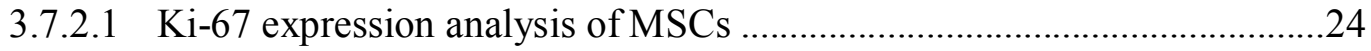

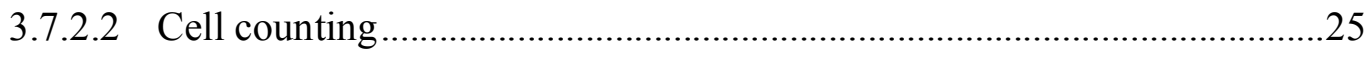

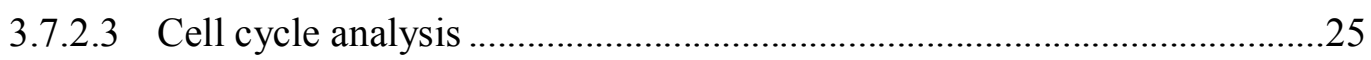

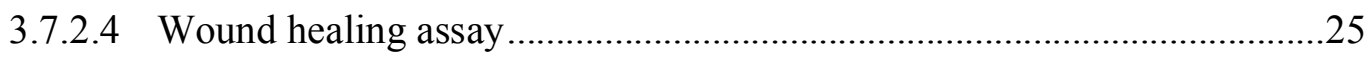

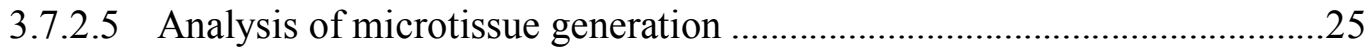

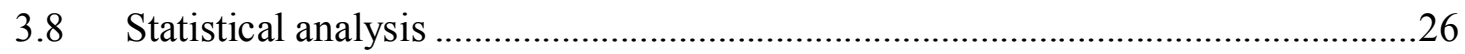

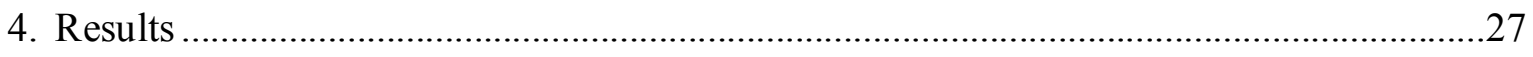

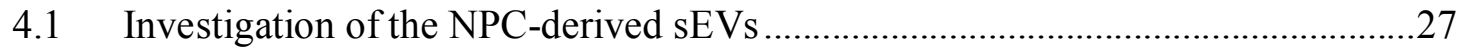

4.1.1 NPC vesicle isolates contained exosome-sized sEVs .................................28

4.1.2 Descriptive statistics of the NPC-derived SEVs released under different

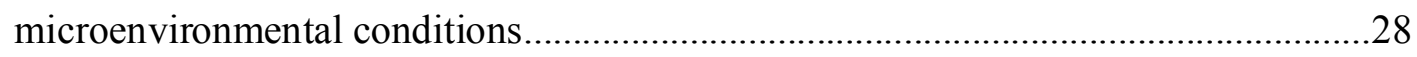

4.1.2.1 $\mathrm{sEV}$ release is elevated under stress conditions.......................................28

4.1.2.2 miRNome of $\mathrm{sEVs}$ is altered under stress conditions ..............................28

4.1.3 In silico analysis of functional differences between $\mathrm{sEV}$ groups ...................29

4.1.3.1 NPC-derived sEVs have cellular and systemic functions as well ............29

4.1.3.2 5-8F sEVs lose their tumour promoting effects, if they are produced in an

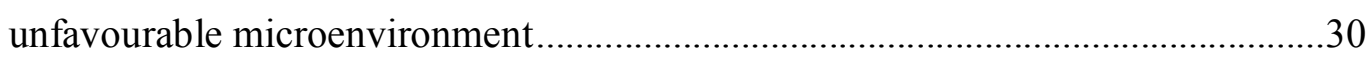

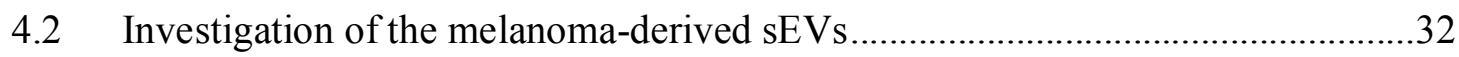

4.2.1 Isolated melanoma vesicles fulfill the minimal experimental requirements for

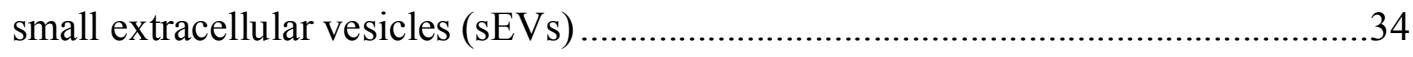


4.2.2 Descriptive statistics of melanoma sEVs released under different microenvironmental conditions .35

4.2.2.1 Vesicle production of melanoma cells is elevated under suboptimal conditions .35

4.2.2.2 Concentration of the encapsulated doxorubicin under cytostatic stress is less than $10 \%$ of median lethal dose 36

4.2.2.3 Encapsulation of $\mathrm{Ag}-\mathrm{TiO}_{2}$ nanoparticles into $\mathrm{sEV}$ s cannot be proven ......36 4.2.2.4 miRNome and proteome of the melanoma sEVs strongly depend on the microenvironmental conditions of the donor cells .37

4.2.3 Comprehensive in silico analysis of functional differences between melanoma sEV groups

4.2.3.1 Melanoma-derived sEVs may affect intracellular, cellular, systemic and immune 'Biofunctions' as well.....

4.2.4 In silico predictions-based in vitro analyses of the melanoma $\mathrm{sEV-induced}$ cellular responses of tumour matrix cells .40

4.2.4.1 Ag- $\mathrm{TiO}_{2} \mathrm{sEVs}$ facilitate proliferation of MSCs. 40

4.2.4.2 Doxo and Ctrl sEVs affect the cell cycle of tumour cells .42

4.2.4.3 Migration capacity of melanoma cells is differently altered by the sEVs 44

4.2.4.4 Each sEV group enhances the migration of endothelial cells..... .45

4.2.4.5 Microtissue generation is facilitated independently of the sEV groups....45

5. Discussion 48

6. New findings .53

7. Conclusions .53

8. References .55

Acknowledgements .68

Supplementary Figures .69

Supplementary Methods. .76 
Abbreviations

AFM

Alix

ANOVA

BMDCs

CL

DAPI

DLS

DMEM

DPBS

EBV

ECM

EMT

EV

FBS

FDR

G1 phase

G2 phase

$\mathrm{H}_{2} \mathrm{O}_{2}$

HNSCC

HSD

Hsp70

IPA

LC-MS/MS

M phase

MAP

MEFs

MISEV2018

MLANA

MMP-(n)

MSC atomic force microscopy

ALG (alpha-1,3-mannosyltransferase) -2 interacting protein $\mathrm{X}$ analysis of variance

bone marrow-derived cells

chemiluminescence

4',6-diamidino-2-phenylindole

dynamic light scattering

Dulbecco's Modified Eagle Medium

Dulbecco's phosphate-buffered saline

Epstein-Barr virus

extracellular matrix

epithelial-mesenchymal transition

extracellular vesicle

fetal bovine serum

false discovery rate

gap1 phase

gap2 phase

hydrogen peroxide

head and neck squamous cell carcinoma

honestly significant difference

70-kDa heat shock protein

Ingenuity Pathway Analysis

liquid chromatography-tandem mass spectrometry

mitosis phase

Molecule Activity Predictor

mouse embryonic fibroblasts

Minimal information for studies of extracellular vesicles 2018

melan-A

matrix metalloproteinase

mesenchymal stem cell 


$\begin{array}{ll}\text { MVB } & \text { multivesicular body } \\ \text { NPC } & \text { nasopharyngeal carcinoma } \\ \text { NTA } & \text { nanoparticle tracking analysis } \\ \text { P/S/A } & \text { Penicillin-Streptomycin-Amphotericin B mixture } \\ \text { PCR } & \text { polymerase chain reaction } \\ \text { qPCR } & \text { quantitative PCR } \\ \text { RT } & \text { room temperature } \\ \text { S phase } & \text { synthesis phase } \\ \text { SD } & \text { standard deviation } \\ \text { SEM } & \text { scanning electron microscopy } \\ \text { SEV } & \text { small extracellular vesicles } \\ \text { SOLiD } & \text { sequencing by oligonucleotide ligation and detection } \\ \text { TEM } & \text { transmission electron microscopy } \\ \text { TME } & \text { tumour microenvironment } \\ \text { Tsg101 } & \text { tumour susceptibility gene 101 }\end{array}$




\section{Introduction}

\subsection{Exosomes, the endosome-derived small extracellular vesicles}

Extracellular vesicles (EVs) are lipid bound vesicles secreted by cells into the extracellular space. They transport cargo, including DNA, RNA, and proteins, between cells ${ }^{1-3}$. The three main types of EVs are apoptotic bodies (ABs), microvesicles (MVs) and exosomes which are differentiated based on their biogenesis, release pathway, size, content and function (Fig. 1) ${ }^{1-4}$. ABs $(1-5 \mu \mathrm{m})$ are released from dying cells, and contain fragmented nuclei and intracellular organelles; MVs (100 nm-1 $\mu \mathrm{m})$ bud directly from the plasma membrane and contain cytoplasmic cargo ${ }^{1-3}$. Exosomes $(30-150 \mathrm{~nm})$ are formed within the endosomal network ${ }^{1-4}$ and three main cellular steps need to be completed to their release; formation of intraluminal vesicles in multivesicular bodies (MVBs); transport of MVBs to the plasma membrane and fusion of MVBs with the plasma membrane. In this process, several molecules, such as endosomal sorting complex required for transport components (ESCRTs), alpha-1,3mannosyltransferase-2 interacting protein $\mathrm{X}$ (Alix), tetraspanins, Ras-associated binding proteins (Rabs) or soluble NSF attachment protein receptors (SNAREs) are implicated ${ }^{5}$.

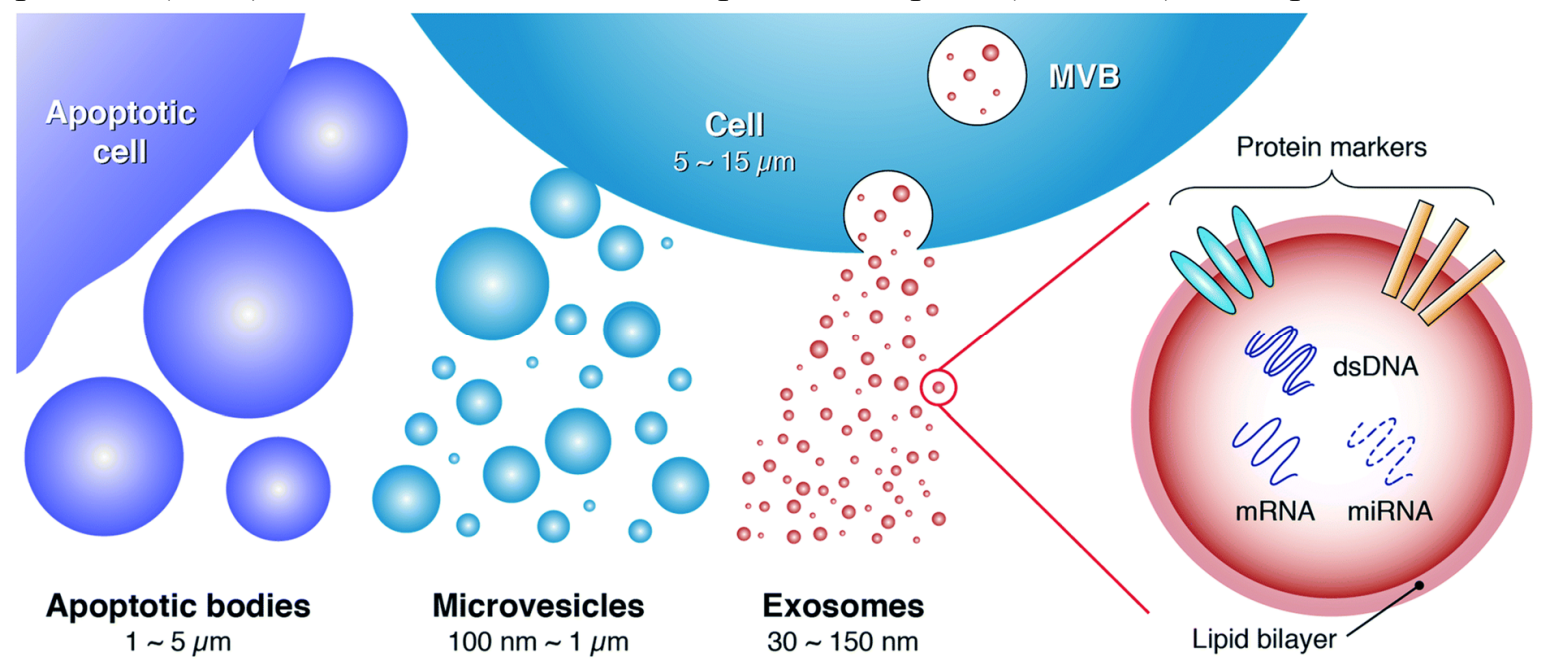

Figure 1. Classification of extracellular vesicles based on size. Exosomes represent a subset of extracellular vesicles with characteristic size in the $30-150 \mathrm{~nm}$ range. Their surface protein markers and nucleic acid cargo play key roles in mediating intercellular communication ${ }^{4}$.

In practice, the isolated material usually contains a mixture of EVs and consensus has not yet emerged on specific markers of EV subtypes. Therefore, members of the International Society for Extracellular Vesicles (ISEV) published a guideline, called Minimal information for studies of extracellular vesicles 2018 (MISEV2018) ${ }^{6}$ to standardise the nomenclature, as well as isolation and analysis protocols of EVs. MISEV2018 suggests the use of operational terms for 
EV subtypes that refer to a physical characteristics, such as size or density; biochemical composition; or releasing conditions. As we isolated EVs based on their size $(<220 \mathrm{~nm})$, I refer to the isolated vesicles here by the term 'small extracellular vesicles' (sEVs), even though their exosomal characteristics, including exosomal shape, size and markers are demonstrated.

Initially, exosomes were proposed to eliminate cellular waste, when in the 1980s Stahl's ${ }^{7}$ and Johnstone's ${ }^{8}$ pioneering work showed that $50 \mathrm{~nm}$ vesicles are released from maturing reticulocytes carrying and externalising transferrin receptor. Over the past three decades, exosomes have surged to the forefront of cell biology research and recently an increasing body of evidence indicates that they play a critical role in the intercellular communication between adjacent as well as distal cells. However, it took decades to replace the image of exosomes as "cellular garbage collectors" with one of exosomes as information conveying vesicles ${ }^{9}$. Today, exosomes considered to be excellently equipped vehicles for information transfer between cells through the horizontal transfer of lipids, proteins and a wide variety of genetic materials, such as DNA, mRNA, and non-coding RNAs (Fig. 1) ${ }^{4}$. Inside the exosomal lipid bilayer, cytokines, miRNAs and other signalling molecules are protected from extracellular enzymes, such as proteases and nucleases. Therefore, the exosomal cargo is delivered to the target cell in a functionally active form ${ }^{10}$.

Exosomes mediate cell-to-cell communication though different mechanisms. They may dock at the plasma membrane of the target cell and activate intracellular signalling by ligandreceptor interaction. Exosomes may be endocytosed by phagocytosis, micropinocytosis or receptor-/raft-mediated endocytosis, and fuse with an endocytic compartment. Exosomes may also be taken up directly by membrane fusion, releasing their content into the cytoplasm ${ }^{11}$.

Clinical relevance of exosomes is also considerable, since they are associated with numerous physiological and pathological conditions, including cancer diseases ${ }^{3}$. Therefore, the exosomemediated molecules and signalling pathways are of intense interest as important new diagnostic and therapeutic targets for personalised medicine ${ }^{4}$.

\subsection{Exosomal communication in the tumour microenvironment}

Tumours are more than insular masses of proliferating cancer cells; they are complex tissues composed of cellular components such as mesenchymal stem cells (MSCs), cancer-associated fibroblasts (CAFs), endothelial cells, immune cells as well as extracellular matrix (ECM) 
components which establish the so called tumour microenvironment (TME) surrounding the tumour cells. The TME does not only surround the tumour cells, it also actively contributes to tumour progression, which requires a continuous paracrine communication ${ }^{12,13}$. One of the possible candidates for intercellular communication might be exosomes, since several recent papers have emphasized the mediating role of exosomes in tumorigenesis, tumour growth, metastasis and immune modulation in the tumour macro- and microenvironment ${ }^{13-15}$. Upon contact with recipient cells, tumour-derived exosomes alter their phenotypic and functional properties conveying molecular and genetic messages ${ }^{9}$.

Exosomes are actively involved in numerous tumorigenic processes at both local and distant

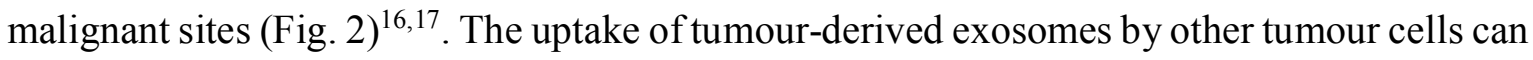
lead to anchorage-independent growth, proliferation and chemoresistance ${ }^{18-20}$. Tumourderived exosomes have also been shown to promote angiogenesis ${ }^{18}$. A number of independent studies have demonstrated that cancer-derived exosomes can be taken up by the endothelial cells resulting their morphological changes, migration, proliferation, tube formation and neovascularization ${ }^{18,21,22}$. Exosomes play a crucial role in the reprogramming of fibroblast cells

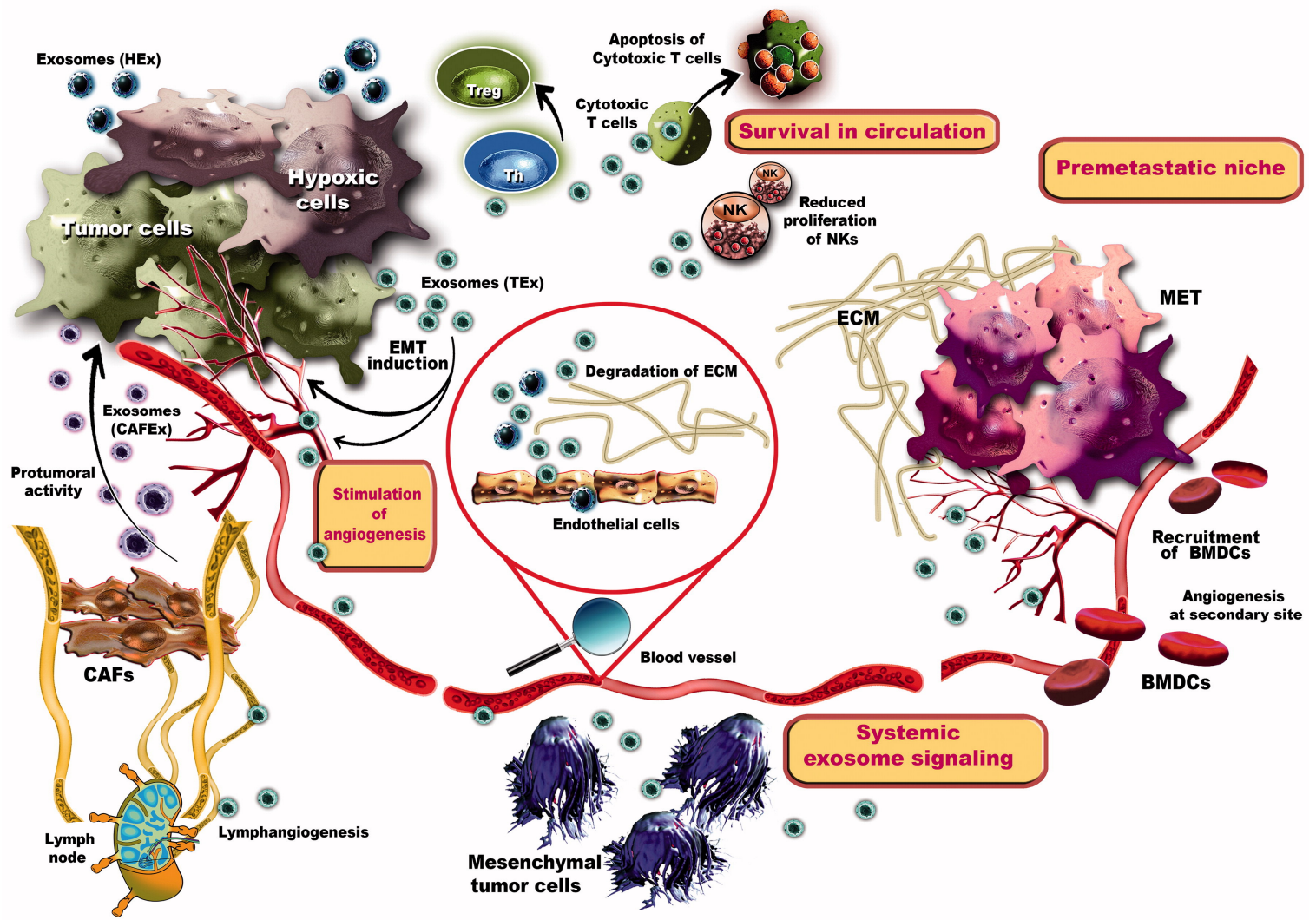

Figure 2. Tumour-derived exosomes and their local and systemic effects. Exosomes alter the TME and prepare distant tissue sites for metastasis ${ }^{16}$. 
and MSCs to promote tumour progression by secreting soluble growth factors, cytokines and chemokines ${ }^{18}$.

TME-produced exosomes may stimulate functions of immune cells or suppress their antitumor activities. For instance, tumour-derived exosomes could inhibit T cell and natural killer (NK) cell activation and promote regulatory $\mathrm{T}$ cell function. They inhibit the activity of dendritic cells and increase the expansion of myeloid-derived suppressor cells (MDSCs). They also stimulate the polarization of macrophages towards the tumour-promoting M2 phase $\mathrm{p}^{9,23}$.

Exosomes are also known to promote metastatic cascade, a series of steps involving tumour cell epithelial-mesenchymal transition (EMT), local invasion, survival and evasion of immune responses, intravasation into the circulation, extravasation at secondary organs and colonisation the metastatic sites ${ }^{24}$. EMT allows tumour cells to acquire mesenchymal properties and ability to migrate out of the primary tumour, invading basement membrane and entering the vasculature. Tumour-derived exosomes seem to regulate cell movement and cell invasion by promoting ECM degradation as well ${ }^{25}$. They facilitate endothelial cell barrier destruction through down-regulation of the tight junction zonula occludens 1 (ZO-1) protein increasing vascular permeability. This perturbation of vascular cell barrier allows tumour cells to intravasate into the circulation and extravasate at secondary sites to form metastatic lesions ${ }^{24}$.

A number of preclinical studies suggest that cancer-derived EVs have a systemic effect on conditioning of secondary tumour sites ${ }^{17}$. Tumour-derived exosomes prepare a pre-metastatic niche, via polarization of tissue macrophages, MSCs and fibroblast cells to an antiinflammatory profile; induction of ECM remodelling; recruitment of bone marrow-derived cells (BMDCs); suppression of immune response; induction of angiogenesis and increase in vascular permeability. After formation of a hospitable microenvironment, tumour cells are able to spawn a secondary tumour growth ${ }^{26}$.

\subsection{Role of exosomes in nasopharyngeal carcinoma}

Nasopharyngeal carcinoma (NPC) is a tumour arising from the epithelial cells that cover the surface and line the nasopharynx. The etiology of NPC seems to follow a multi-step process, in which Epstein-Barr virus (EBV), ethnic background, and environmental carcinogens all seem to play an important role ${ }^{27}$. It is a heterogenous group of carcinomas, the World Health 
Organization (WHO) distinguishes three histological patterns, (i) type I, keratinizing squamous-cell carcinoma; (ii) type II, differentiated non-keratinizing carcinoma; and (iii) type III, undifferentiated carcinoma. NPC is the commonest epithelial cancer in adults, its local recurrence and metastasis to cervical lymph nodes are the main cause of mortality, but understanding the pathogenesis of NPC is still in the infancy ${ }^{28}$.

Recently, it was shown, that NPC-related exosomes promote tumour growth and progression through complex mechanisms. Multiple exosomal components are involved in cell proliferation, apoptosis, adhesion and invasion ${ }^{29}$. Exosomes may act as an important mediator in the EBV-tumorigenic progress as wel1 ${ }^{29-32}$. Exosomes released by EBV-infected NPC cells contain viral product, the latent membrane protein 1 (LMP1), which can induce broad range of anergic effects on immune cells such as the inhibition of $\mathrm{T}$ cell proliferation, NK cytotoxicity, and interferon- $\gamma\left(\right.$ IFN- $\gamma$ ) release ${ }^{33}$. LMP1 was also shown to increase exosomal hypoxiainducible factor- $1 \alpha$ (HIF-1 $\alpha)$, which can trigger an EMT program in neighbouring tumour cells $^{34}$. NPC-derived exosomes have also been shown to contain matrix metalloproteinase-13 (MMP-13), which are known to digest the ECM and facilitate the protrusion of invasive cancer cells ${ }^{35}$. In addition, they carry the oncogenic HS1-associated protein X-1 (HAX-1), which can accelerate tumour growth and angiogenesis ${ }^{36}$.

\subsection{Role of exosomes in malignant melanoma}

Melanoma is the malignant transformation of melanocytes, the melanin-producing neural crest-derived cells of the epidermis. Its risk factors include ultraviolet radiation; presence of melanocytic or dysplastic naevi; personal and family history of melanoma; high socioeconomic status; special phenotypic characteristics, such as fair hair, eye and skin colours or the tendency to freckle ${ }^{37}$. Malignant melanoma is one of the most aggressive cancers, it has the potential to disseminate from a relatively small primary tumour (less than $2 \mathrm{~mm}$ thick) and metastasize to multiple sites, including the lung, liver, brain, bone and lymph nodes ${ }^{38}$. This high metastatic potential can be explained by unique features of melanoma. For instance, melanoma cells are mesenchymal in nature ensuring that larger percentage of cells can act as stem cells with selfrenewal capacity. They share many antigens with vascular endothelial cells (vasculogenic mimicry) allowing not only their survival in the circulation, but increased migration and invasion capacity as well. 
The unique composition of exosomes released from melanoma cells may also contribute to their high invasiveness ${ }^{38}$. Exosomal miR-222 has been shown to play a tumorigenic role in melanoma, by its ability to induce the PI3K/AKT (phosphatidylinositol 3-kinase/RAC-alpha serine/threonine-protein kinase) pathway ${ }^{39}$. Transferring pro-angiogenic factors, such as interleukin-8 (IL-8), vascular endothelial growth factor (VEGF) and MMP-2, melanomaderived exosomes promote neo-angiogenesis ${ }^{40}$. Exosomal miR-9 further support this process, by inducing the JAK-STAT (Janus kinase/signal transducer and activator of transcription) pathway in the endothelial cells ${ }^{41}$. Exosomal MMP-14 and ADAM (a disintegrin and metalloproteinase) proteases promote the remodelling and degradation of the $\mathrm{ECM}^{42,43}$. Exosomes may also re-educate BMDCs through horizontal transfer of the oncoprotein receptor tyrosine kinase MET (MNNG HOS transforming gene), which enhances the mobilisation of BMDCs and thus facilitates their recruitment to metastatic sites ${ }^{44}$, where BMDCs establish a suitable microenvironment for trapping circulating melanoma cells ${ }^{45}$. Beside other immunomodulatory effects, melanoma-derived exosomes has been shown to carry programmed cell death-ligand1 (PD-L1) on their surface, which suppresses the function of CD8+ T cells and facilitates tumour growth ${ }^{43,46}$. Exosomal PD-L1 has recently been suggested as a predictor for the clinical outcomes of anti-programmed cell death-1 (PD-1) therapy ${ }^{46}$.

\subsection{Role of intrinsic microenvironmental factors in the exosome-mediated communication of tumour cells}

Most of the solid tumours develop a hostile tumour microenvironment associated with the expansion of hypoxic and necrotic areas as the existing vasculature cannot fulfill the increasing oxygen demand of rapidly expanding tumours ${ }^{47}$. These areas have a special chemical microenvironment with low oxygen, low $\mathrm{pH}$ and low nutrient concentration. Intrinsic stress conditions, such as hypoxia and acidosis increase exosome release from malignant cells and lead to TME alteration and expansion, which subsequently results in tumour progression ${ }^{48,49}$. Thus, content analysis of the exosomes may reveal their function in TME progression in malignancies, and this may further lead to developing more efficient sEV-based strategies for cancer prognosis and therapy ${ }^{50}$. 


\subsection{Effects of different therapeutic conditions on the exosome-mediated communication of tumour cells}

Chemotherapy provides long-term clinical benefits to patients, but it may induce tumourpromoting host responses as well ${ }^{51}$. Tumour cells may adapt to the chemotherapy-induced stress conditions, which is reflected in their exosomal communication. Transmission of extracellular vesicles in the TME promotes development of chemoresistance in tumour cells and transfer of drug resistance traits ${ }^{52}$. Furthermore, Keklikoglou et al. have shown that paclitaxel and doxorubicin elicit the production of pro-metastatic breast cancer-derived EVs ${ }^{53}$; König et al. investigated circulating EV levels of breast cancer patients and an increased EV concentration before neoadjuvant chemotherapy was associated with therapy failure, and an elevated EV concentration after neoadjuvant chemotherapy was associated with a reduced three-year progression-free and overall survival ${ }^{54}$.

Hyperthermia treatment involves increasing the target site temperature to induce thermic stress which results in cancer cell cytotoxicity and immune response stimulation via immune cell activation. Therefore, hyperthermia may enhance the therapeutic efficacy in combined therapies $^{55,56}$, but its impact on tumour exosome-mediated intercellular communication has not been described yet.

In case of oxidative stress-based therapies, such as radiotherapy or photodynamic therapy, Mutschelknaus et al. have linked radiation-induced changes of exosomes to their ability to promote migration of recipient head and neck squamous cell carcinoma (HNSCC) cells ${ }^{57}$. Exosomes released from irradiated cells have been also shown to mediate the radiation-induced bystander effect, which means that non-irradiated cells in the close vicinity of oxidative stressdamaged cells are induced to exhibit a similar phenotype ${ }^{58,59}$. Oxidative stress can be also induced by photocatalyst nanoparticles, such as $\mathrm{Ag}-\mathrm{TiO}_{2}{ }^{60,61}$. During the process of photocatalysis under appropriate (exciting) wavelength, reactive hydroxyl radicals $(\mathrm{OH} \cdot)$ are produced, which are primarily responsible for photo-oxidation of organic materials or inactivating bacteria ${ }^{62}$. $\mathrm{OH}$ - radicals are the most reactive oxygen species and cause irreversible DNA damage which can lead to DNA degradation in bacteria ${ }^{62}$. In our previous work, the amount of reactive $\mathrm{OH} \cdot$ radicals formed on $\mathrm{Ag}-\mathrm{TiO}_{2}$ particles was determined by the hydrogen peroxide $\left(\mathrm{H}_{2} \mathrm{O}_{2}\right)$-induced luminol-dependent chemiluminescence $(\mathrm{CL})$ reaction ${ }^{63}$. It was presented that concentration of the $\mathrm{Ag}-\mathrm{TiO}_{2}$-produced $\mathrm{OH} \cdot$ radicals was equivalent to 
$0.33 \mathrm{mM} \mathrm{H}_{2} \mathrm{O}_{2}$ after 20 min visible light illumination. $\mathrm{Ag}-\mathrm{TiO}_{2}$ nanoparticles represent a high potential for therapeutic applications through antibacterial ${ }^{60,63}$, antifungal ${ }^{64}$ and anticancer ${ }^{65}$ activities. However, the effects of $\mathrm{Ag}-\mathrm{TiO}_{2}$-based therapies on the vesicular communication have not been investigated yet.

\section{Aims}

In this study, we aimed to compare the vesicular information transfer of tumour cells under different microenvironmental conditions using a unique approach. sEVs are complex information packages, and we consider them as message delivering units with specific molecular patterns, rather than putting emphasis on a few vesicular signal molecules.

In the first part of this project, we investigated the 5-8F human NPC cell line-derived vesicles under cytostatic and oxidative stress. In the second part, B16F1 mouse melanoma-derived sEVs were analysed after cytostatic, heat and oxidative stresses as well.

We aimed to perform a comprehensive quantitative and qualitative comparison of the tumourderived sEVs. Our specific aims were

1. to compare the sEV release under different microenvironmental conditions,

2. to describe the molecular pattern of SEV groups, which were released under various microenvironmental conditions,

3. to investigate potential doxorubicin and $\mathrm{Ag}-\mathrm{TiO}_{2}$ transfer by sEVs,

4. to establish an in silico model for the functional activity of sEVs in the recipient cells,

5. to compare functional properties of sEV groups, which were released under various microenvironmental conditions,

6. to compare the adaptivity of sEV-mediated communication of different tumour cells,

7. to describe the response patterns of sEV recipient cells, induced by the melanoma-derived sEV groups,

8. to compare the cellular migratory response to the melanoma-derived sEVs in different type of recipient cells,

9. to determine the utility of in silico analyses in the sEV research. 


\section{Materials and methods}

\subsection{Cell cultures}

5-8F human NPC cell line was kindly provided by Ji Ming Wang (National Cancer Institute, Frederick, MD, USA) and cultured in Dulbecco's modified Eagle's medium (DMEM) supplemented by $10 \%$ fetal bovine serum (FBS; Euroclone SpA), 1\% minimum essential medium (MEM) non-essential amino acids, 1\% MEM vitamin solution and 1\% PenicillinStreptomycin-Amphotericin B mixture (P/S/A; all from Lonza).

B16F1 (ECACC 92101203) mouse melanoma cell line was obtained from the European Collection of Authenticated Cell Cultures (ECACC) and cultured in DMEM supplemented by $10 \%$ FBS, 2 mM L-glutamine and 1\% P/S/A.

For sEV production of both cell lines, media were prepared using vesicle-depleted FBS.

Primary mouse mesenchymal stem cells (MSCs) were isolated from adipose tissue of 6-8 week old male C57BL/6N mice (Charles River Laboratories), maintained using the MesenCult Expansion Kit (Stemcell Technologies) in a humidified incubator at $37^{\circ} \mathrm{C}$ and $5 \% \mathrm{CO}_{2}$. Purity of MSC cultures were checked by flow cytometry using the Mouse Multipotent Mesenchymal Stromal Cell Marker Antibody Panel (R\&D Systems) and a BD FACSCalibur instrument coupled with BD CellQuest Pro 6.0 (BD Biosciences). Animal experiments were performed in accordance with the national and European animal ethics guidelines. The animal experimental protocols were approved by the Animal Experimentation and Ethics Committee of the Biological Research Centre and the Hungarian National Animal Experimentation and Ethics Board (clearance number: XVI./78/2018).

Mouse embryonic fibroblasts (MEFs; ATCC SCRC-1040) were obtained from the American Type Culture Collection (ATCC) and cultured in DMEM supplemented by 15\% FBS and $1 \% \mathrm{P} / \mathrm{S} / \mathrm{A}$.

BEnd.3 mouse endothelial cell line (ATCC CRL-2299) was obtained from the ATCC and cultured in DMEM supplemented by $10 \% \mathrm{FBS}$ and $1 \% \mathrm{P} / \mathrm{S} / \mathrm{A}$.

All cell cultures were maintained at $37^{\circ} \mathrm{C}$ in a humidified incubator with $5 \% \mathrm{CO}_{2}$. 


\section{2 $\mathrm{Ag}-\mathrm{TiO}_{2}$ photocatalyst particles}

For synthesis of the $\mathrm{Ag}-\mathrm{TiO}_{2}$ nanoparticles, commercially available $\mathrm{TiO}_{2}$ (Degussa $\mathrm{P} 25$ from Evonik) photocatalyst with a specific surface area of $\sim 50 \mathrm{~m}^{2} / \mathrm{g}$ was used as a standard photocatalyst without any treatment. Plasmonic Ag nanoparticles were prepared on the surface of $\mathrm{TiO}_{2}$ in order to enhance the photocatalytic efficiency of the prepared $\mathrm{Ag}-\mathrm{TiO}_{2}$ sample. The prepared $\mathrm{Ag}-\mathrm{TiO}_{2}$ photocatalyst contained 0.5 wt.\% surface silver nanoparticles ${ }^{60,61}$. Dispersion of particles was made in DPBS at a concentration of $10 \mathrm{mg} / \mathrm{ml}$ and sonicated for 30 min directly before use.

\subsection{Stress conditions}

$5-8 \mathrm{~F}$ cell cultures were treated at $70 \%$ confluency in three different ways: the medium was replaced with (i) fresh medium, (ii) fresh medium supplemented by $0.6 \mu \mathrm{M}$ doxorubicin,

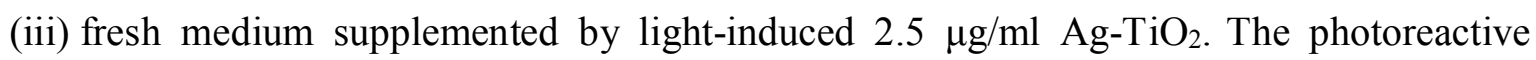
$\mathrm{Ag}-\mathrm{TiO}_{2}$ nanoparticles were induced by a low-pressure mercury lamp $(\lambda \geq 360 \mathrm{~nm}$, GCL303T5/4 type, LightTech) for $60 \mathrm{~min}$ from $3 \mathrm{~cm}$ distance in a $75 \%$ medium volume to avoid the light absorption and reflection of the medium layer. After the illumination, the medium was adjusted to the final volume.

In case of B16F1 cell cultures 2 additional culture conditions were applied. One group of cultures were exposed to heat stress by incubating the cells at $42^{\circ} \mathrm{C}$ for $2 \mathrm{~h}$ in every $24 \mathrm{~h}$ (a total of 3 times). The other group was a control of the $\mathrm{Ag}-\mathrm{TiO}_{2}$ treatment. To eliminate the effect of illumination itself, these cell cultures received medium illuminated in the same way as described above (Table 1).

Parameters of the cytostatic and oxidative stresses were based on previous optimisation by proliferation assay. Heat stress conditions were adapted from literature data ${ }^{66}$.

In each group, $72 \mathrm{~h}$ supernatants of 6 parallel cell cultures were harvested, pooled and subjected to sEV isolation.

\subsection{Scanning electron microscopy (SEM)}

B16F1 cells seeded to poly-L-lysine-coated $5 \mathrm{~mm}$ cover glasses were treated as described above. After 24 h, cells were washed with Dulbecco's phosphate-buffered saline (DPBS) and fixed for overnight in $2.5 \%$ glutaraldehyde and $0.05 \mathrm{M}$ cacodylate buffer. Then, cells were washed with DPBS, dehydrated with a graded ethanol series $(30 \%, 50 \%, 70 \%, 80 \%$, 
each for $1 \mathrm{~h}$ and $100 \%$, for $3 \times 1 \mathrm{~h}$ ) and dried with a critical point dryer (Quorum, K850). Cover glasses were mounted onto microscope stubs using carbon tape, followed by $15 \mathrm{~nm}$ gold coating (Quorum Q150) and observed under a field-emission scanning electron microscope (Jeol, JSM-7100F/LV). Images were taken in 1,500× and 20,000× magnification.

\subsection{SEV isolation and characterisation}

Vesicles were isolated by differential filtration and ultracentrifugation. Briefly, supernatants were centrifuged at $780 \mathrm{~g}$ for $5 \mathrm{~min}$, at $3,900 \mathrm{~g}$ for $15 \mathrm{~min}$ at $4{ }^{\circ} \mathrm{C}$, then filtered through a $0.22 \mu \mathrm{m}$ pore-size membrane to remove cells, debris and larger vesicles. Small EVs were pelleted by ultracentrifugation at $150,000 \mathrm{~g}$ for $60 \mathrm{~min}$ at $4{ }^{\circ} \mathrm{C}$ using a T-1270 fixed-angle rotor and a $\mathrm{WX}+$ ultracentrifuge (Sorvall). The pellet was washed twice and re-suspended in DPBS. Protein concentrations of B16F1 sEV isolates were measured by the Pierce BCA (bicinchoninic acid) Protein assay kit (Thermo Scientific) on a benchtop microplate reader (Multiskan RC, Thermo Scientific) coupled with the Ascent Software 2.6. Small EVs were characterised by atomic force microscopy (described below), dynamic light scattering using a Zetasizer Nano S instrument (Malvern Panalytical) and Western blot analysis (described below).

\subsubsection{Atomic force microscopy (AFM)}

AFM measurements were carried out with an Asylum MFP-3D head and controller (Asylum Research). The driver program MFP-3D Xop was written in IGOR Pro Software (Wavemetrics). For imaging, gold coated silicon nitride rectangular cantilevers were used with a typical spring constant of $0.03 \mathrm{~N} / \mathrm{m}$ in air (BL-RC150 VB, Olympus). The spring constant for each cantilever was determined by thermal calibration followed by Sader's method ${ }^{67-70}$. For the measurements, freshly cleaved $1 \times 1 \mathrm{~cm}$ mica (SPI-Chem Mica Sheets) surfaces were used as supports for sEVs. The negatively charged mica surfaces were incubated in $2 \%$ APTES ((3-aminopropyl)triethoxysilan, Sigma) dissolved in isopropanol for $90 \mathrm{~min}$ in shaking condition at room temperature (RT) to create free amine groups on their surface ${ }^{69,71,72}$. sEVs were attached to the modified surface with glutaraldehyde. Measurements were carried out in tapping (AC) mode in DPBS. Typically, $512 \times 512$ point scans were taken at $0.4 \mathrm{~Hz}$ scan rate. Both the trace and retrace images were recorded and compared. Experiments were repeated 3-5 times. 


\subsubsection{Western blot (WB) assay}

Vesicular pellets were re-suspended in NP40 lysis buffer (Invitrogen) and incubated on ice for $30 \mathrm{~min}$. Protein content of sEV isolates were measured as described above and $25 \mu \mathrm{g}$ protein of each sample was diluted in a mixture of $4 \times$ sample buffer (NuPAGE LDS Sample Buffer) and $10 \times$ reducing agent (NuPAGE Sample Reducing Agent), boiled at $96^{\circ} \mathrm{C}$ for $10 \mathrm{~min}$, immediately cooled on ice and separated beside a ProSieve color protein marker (Lonza) on 4-12\% Bis-Tris Protein Gel (NuPAGE Novex) using an XCell SureLock Mini-Cell (Invitrogen) at $200 \mathrm{~V}$ and $0.03 \mathrm{~A}$ for $40 \mathrm{~min}$ with an electrophoresis buffer (NuPAGE MOPS SDS Running Buffer). Then, proteins were transferred to an Immobilon-P polyvinylidene difluoride (PVDF) membrane (Millipore) using an XCell SureLock Mini-Cell (Invitrogen) at $30 \mathrm{~V}$ and 10-170 $\mathrm{mA}$ for $60 \mathrm{~min}$ with a transfer buffer (NuPAGE). Blocking was performed by $5 \%$ non-fat milk in Tris-buffered saline (TBS)-Tween solution (TBST) buffer ( $25 \mathrm{mM}$ Tris-HCl, $150 \mathrm{mM} \mathrm{NaCl}, 0.05 \%$ Tween-20, $\mathrm{pH} 7.2$ ) for $60 \mathrm{~min}$ at RT. For detection of the vesicular markers, we used rabbit anti-mouse CD9 (1:500, LifeSpan Biosciences), rabbit anti-mouse CD63 (1:500, Santa Cruz), mouse HSP70/HSP72 (70/72-kDa heat shock protein) monoclonal antibody (1:8,000, Enzo Life Sciences), rabbit anti-mouse Alix (1:500, SigmaAldrich), rabbit anti-mouse TSG101 (tumour susceptibility gene 101) (1:2,500, SigmaAldrich), rabbit anti-mouse Calnexin (1:1,000, Abcam) and rabbit MLANA (melan-A) polyclonal antibody $(1: 2,000$, ABclonal). Primary antibody labelling was performed in $1 \%$ non-fat milk in TBST buffer overnight at $4^{\circ} \mathrm{C}$. Unbound antibodies were removed by washing 3 times for $10 \mathrm{~min}$ in TBST buffer. As secondary antibodies, horseradish peroxidase (HRP)-conjugated anti-rabbit IgG and anti-mouse IgG (R\&D Systems) were used in 1\% non-fat milk-containing TBST buffer with 60 min incubation at RT. After washing, protein bands were visualised by the WesternBright ECL detection kit (Advansta) and an Odyssey Fc imaging system (LI-COR) coupled with Image Studio Lite 5.2.

\subsubsection{Quantitative comparison of $\mathrm{sEV}$ groups}

Released number of sEVs was determined by nanoparticle tracking analysis (NTA) using a NanoSight NS500 instrument (Malvern Panalytical) based on the manufacturer's protocol. 


\subsubsection{Fluorescence spectroscopy}

The emission and excitation spectra of doxorubicin were measured by a FluoroLog-3 fluorescence spectrometer (Horiba). The maximum wavelengths for excitation $\left(\lambda_{\mathrm{ex}}=492 \mathrm{~nm}\right)$ and emission $\left(\lambda_{\mathrm{em}}=592 \mathrm{~nm}\right)$ were then used for measuring the fluorescence intensity of Doxo sEVs and Ctrl sEVs as a background. A calibration curve of doxorubicin covering a concentration range of $0-1,000 \mathrm{nM}$ was applied to determine the encapsulated doxorubicin concentration of the sEV samples.

\subsubsection{Dynamic light scattering (DLS) measurements of $\mathrm{Ag}-\mathrm{TiO}_{2}$ particles}

The particle size values of the $\mathrm{Ag}-\mathrm{TiO}_{2}$ photocatalyst particles were determined by DLS with a Zetasizer Nano ZS ZEN 4003 apparatus (Malvern Panalytical) equipped with a He-Ne laser $(\lambda=633 \mathrm{~nm})$. The measurements were performed in B16F1 culture medium for a $72 \mathrm{~h}$ time interval. Size distribution measurements were carried out in triplicate, and mean \pm standard deviation (SD) values are reported.

\subsubsection{Chemiluminescence (CL) detection of $\mathrm{Ag}-\mathrm{TiO}_{2}$ nanoparticles}

Isolated $\mathrm{Ag} \mathrm{Ctrl}$ and $\mathrm{Ag}-\mathrm{TiO}_{2}$ sEVs were lysed by Tris-EDTA-NaCl-Triton X-100 (TENT) buffer and freeze-thaw cycle, then a 10-fold, 5-step $\mathrm{Ag}-\mathrm{TiO}_{2}$ nanoparticle dilution series $(0-2.5 \mu \mathrm{g} / \mathrm{ml})$ was prepared in Ag Ctrl sEV suspension for calibration. Protein concentration of sEV lysates was $200 \mu \mathrm{g} / \mathrm{ml}$. After 30 min illumination on 96 -well plates, $50 \mu 13.38 \mathrm{mM}$ luminol solution was added to $50 \mu \mathrm{l}$ of samples and light emission was immediately detected by a Luminoskan Ascent Microplate Luminometer (Thermo Scientific). Each sample was measured in triplicates.

\subsubsection{Transmission electron microscopy (TEM)}

The morphology of $\mathrm{Ag}-\mathrm{TiO}_{2} \mathrm{sEVs}$ and $\mathrm{Ag}-\mathrm{TiO}_{2} \mathrm{sEVs}$ mixed with $\mathrm{Ag}-\mathrm{TiO}_{2}$ nanoparticles was examined using a Tecnai G2 20 X-Twin type instrument (FEI), operating at an acceleration voltage of $200 \mathrm{kV}$. For TEM measurements the samples were dropped on a grid (carbon film with 200 Mesh coper grids; CF200-Cu, Electron Microscopy Sciences) and dried.

\subsection{Analysis of the cargo of $\mathrm{SEV}$ groups}

MiRNomes of sEVs were described for both cell types, and proteomes of sEVs were determined only for B16F1 groups. 


\subsection{1 miRNA analysis of SEVs}

Pellets of sEVs were subjected to miRNA isolation using the NucleoSpin miRNA isolation kit (Macherey-Nagel) according to the manufacturer's instructions. Sequencing was performed using sequencing by oligonucleotide ligation and detection (SOLiD) Total RNA-Seq lit for Small RNA Libraries (Applied Biosystems) based on the manufacturer's protocol. Purification was performed on 10\% Tris base/boric acid/EDTA (TBE)-Urea gels stained with Sybr Gold nucleic acid gel stain (both from Invitrogen). Final purification was performed using PureLink polymerase chain reaction (PCR) Micro Kit (Invitrogen). Final libraries were quality checked using High Sensitivity DNA kit on Bioanalyzer (Agilent Technologies). Concentration of each library was determined using the SOLiD Library TaqMan Quantitation Kit (Life Technologies). Each library was clonally amplified on SOLiD P1 DNA Beads by emulsion PCR (ePCR). Emulsions were broken with butanol, and ePCR beads enriched for templatepositive beads by hybridization with magnetic enrichment beads. Template-enriched beads were extended at the $3^{\prime}$ end in the presence of terminal transferase and 3 ' bead linker. Beads with the clonally amplified DNA were deposited onto SOLiD sequencing slide and sequenced on SOLiD 5500xl instrument using the 50-base sequencing chemistry.

Bioinformatics analysis of raw data, quality assessment, read trimming, read mapping and miRNA expression profiling was carried out in CLC Genomics Workbench 8.0.2 (Qiagen Bioinformatics) using annotated Mus musculus miRNA sequences according to the miRBase release 21 as a mapping reference. Only miRNAs with $\geq 10$ read counts were accepted.

In case of B16F1-derived sEVs, results of sequencing were validated by quantitative PCR (qPCR) on 3 selected miRNAs, mmu-miR-16-5p, mmu-miR-125b-5p, mmu-miR-29a-3p. Intact total RNA, including miRNA were prepared from sEV isolates by miRNA Miniprep System (Promega) according to the manufacturer's instructions. Then, $70 \mathrm{ng}$ of each samples were reverse transcribed using microRNA cDNA synthesis kit (Sigma-Aldrich). The qPCR reactions were performed on a PikoReal Real-Time PCR System (Thermo Scientific) using SYBR Green chemistry and commercially available miRNA specific primers (Sigma-Aldrich). $\mathrm{Cq}$ values of each miRNAs were normalized by U6 endogenous controls in all samples and expression levels were calculated using -ddCt method. 


\subsubsection{Protein analysis of B16F1-derived sEVs}

Detailed liquid chromatography-tandem mass spectrometry (LC-MS/MS) analysis of sEVs is described in the Suppl. Methods. Briefly, $25 \mu \mathrm{g}$ of vesicular proteins were separated by sodium dodecyl sulphate-polyacrylamide gel electrophoresis (SDS-PAGE) and stained with Coomassie blue. Then each lane was cut to 12 equal bands and subjected to an in-gel trypsinisation procedure. The extracted peptides were analysed on an LTQ-Orbitrap Elite (Thermo Scientific) mass spectrometer on-line coupled with a nano-high-performance liquid chromatography (nanoHPLC; nanoAcquity, Waters) system. Searchable peaklists were extracted using Proteome Discoverer 1.4 (Thermo Scientific) and subjected to database search on our in-house Protein Prospector 5.14.1 search engine against the Mus musculus and Bos taurus protein sequences of the Uniprot database. Proteins were accepted if they was identified with $\geq 3$ unique peptides, but peptides with identical bovine and mouse sequence were excluded. False discovery rate (FDR) values were less than $1 \%$ in all cases. Results were validated on the MLANA and HSP70 proteins by Western blot as described above.

\subsection{Functional comparison of the SEV groups}

Bioinformatics analyses were performed on the 5-8F miRNA data and the B16F1 miRNA and protein data. Results of the in silico analysis were verified by in vitro functional assays using the B16F1 sEV isolates.

\subsubsection{In silico analysis of $\mathrm{SEV}$ cargos}

Normalised miRNA and protein data derived from the SOLiD sequencing and LC-MS/MS were analysed by the Ingenuity Pathway Analysis (IPA, Qiagen Bioinformatics). First, we used the 'Core Analysis' feature to reveal functional differences between the sEV groups, where 'Top 5 canonical pathways' and 'Top 5 molecular and cellular functions' were obtained. Secondly, using the 'Comparison Analysis' feature, we created a heatmap containing 'Biofunctions', which had relevance in NPC or melanoma and $p \leq 0.00001$. Heatmap of melanoma sEVs was divided into four parts based on biological relevance; intracellular, cellular, systemic and immune processes were selected into separated panels. Thirdly, some 'Biofunctions' were chosen for further investigation to reveal the regulatory effects of sEVs on them. Using the 'Grow' tool, the upstream interacting vesicular molecules were identified for selected 'Biofunctions' for each sEV group. Then, using the 'Molecule Activity Predictor (MAP)' tool, we could reveal the activation or inhibitory effects of each sEV group for each 
'Biofunction'. Through these in silico analyses, we were able to model the effects of the different sEVs in the recipient cells in spite of their molecular complexity. Figures were edited in the IPA 'Path Designer' and completed with Excel diagrams. For all IPA analyses, the confidence level was set to 'Experimentally observed' enabling literature data-based analysis, but not unproven predictions.

\subsubsection{In vitro assays for functional comparison of the B16F1 sEV groups}

To avoid additional effects of changing conditions, cells were exposed to sEVs in their standard, complete media before each of the following functional assays. Briefly, cells were treated with $200 \mu \mathrm{g} / \mathrm{ml} \mathrm{sEV} \mathrm{suspensions,} \mathrm{or} \mathrm{DPBS} \mathrm{as} \mathrm{a} \mathrm{negative} \mathrm{control} \mathrm{for} 24 \mathrm{~h}, 48 \mathrm{~h}$ or $72 \mathrm{~h}$. For longer incubation times, treatments were repeated in every $24 \mathrm{~h}$. The functional effects of sEVs on the recipient cells were investigated in 5 different ways, described below.

\subsubsection{Ki-67 expression analysis of MSCs}

MSCs exposed to sEVs for $24 \mathrm{~h}$ and $72 \mathrm{~h}$ were fixed in 4\% paraformaldehyde (PFA) for $10 \mathrm{~min}$ at RT for immunocytochemistry. Then, cells were permeabilised with $0.1 \%$ Triton X-100 and non-specific antibody binding was blocked with $5 \%$ bovine serum albumin (BSA). We applied direct labelling using anti-mouse/rat Ki-67 monoclonal antibody conjugated to eFluor 615 dye (1:400, eBioScience) in $1.2 \%$ BSA overnight at $4{ }^{\circ} \mathrm{C}$. Nuclear counterstaining was performed with 4',6-diamidino-2-phenylindole (DAPI) for $15 \mathrm{~min}$ at RT. Cells were washed 3 times with DPBS for $5 \mathrm{~min}$ between each step. Finally, the cells were covered by Fluoromount-G (SouthernBiotech) and cover glasses. Fluorescent images were taken by an Operetta high content imaging system (PerkinElmer) and analysed by an image analysis and machine learning software (SCT Analyzer 1.0) developed by the Single-Cell Technologies $\operatorname{Ltd}^{73}$. Our pipeline was comprised of cell segmentation, feature extraction and machine learning modules. K-means algorithm was used for the nuclei segmentation based on the DAPI signal, then we extracted the eFluor 615 signal-related features, i.e. max intensity, min intensity, mean intensity, median intensity and SD intensity for the generated nuclei masks. We established a training set with two classes for the Ki-67 positive and the negative cells. This training set containing 100 objects in both classes was validated by the implemented k-fold crossvalidation. For machine learning, we used the Multi-Layer Perceptron (MLP) method. The Ki-67 expression analysis was repeated 4 times and the applied methods enabled to analyse a total of 159,596 cells. 


\subsubsection{Cell counting}

MSC cultures in 384-well plates were exposed to sEVs for $24 \mathrm{~h}$ and $72 \mathrm{~h}$, then fixed in $4 \%$ PFA for $10 \mathrm{~min}$ at RT and stained with $1 \mu \mathrm{g} / \mathrm{ml}$ DAPI for $15 \mathrm{~min}$ at RT. Images were acquired about the whole wells using a TCS SP8 microscope (Leica) in fluorescent mode, followed by an analysis using the SCT Analyzer 1.0 machine learning software. The experiment was repeated 3 times.

\subsubsection{Cell cycle analysis}

Changes in the cell cycle dynamics of SEV-exposed B16F1 cells were analysed using the Cell-Clock cell cycle assay (Biocolor) according to the assay protocol. This assay can be used to distinguish the four major phases of the mammalian cell cycle using a vital redox dye, which is yellow, green or dark blue in gap1 (G1), synthesis/gap2 (S/G2), and mitosis (M) phase cells, respectively. After staining, cells were photographed using an Axiovert S100 microscope (Zeiss) equipped by a Nikon D5000 camera. Images were analysed by the ImageJ software to determine the percentage of cells in each cell cycle phase. The experiment was performed with cells exposed to sEVs for $24 \mathrm{~h}, 48 \mathrm{~h}$ and $72 \mathrm{~h}$ with 4 repeats.

\subsubsection{Wound healing assay}

Alterations in the migration capabilities of sEV-exposed B16F1 cells and bEnd.3 cells were assessed by scratch assay. Nearly confluent monolayers of cells were scratch wounded using a sterile $200 \mu 1$ pipette tip, washed 3 times with culture media to remove cellular debris, then treated with $200 \mu \mathrm{g} / \mathrm{ml} \mathrm{sEV}$ suspensions or DPBS in fresh media. Wound closure was followed until the cell-free area decreased below $10 \%$ in at least 1 sample, when images were taken by an inverted microscope (Zeiss, Axiovert S100) equipped by a Nikon D5000 camera. Greyscaled images were analysed using the MRI Wound Healing Tool in the ImageJ software. The experiment was repeated 8 times for B16F1 cells and 4 times for bEnd. 3 cells.

\subsubsection{Analysis of microtissue generation}

Effects of different sEVs on cell-cell contact and cell-ECM interactions was examined on MSC-B16F1 and MEF-B16F1 co-cultures using a simplified 3D tumour matrix model to better represent the in vivo conditions, then 2D cultures. Equal number of MSC/MEF and B16F1 cells were seeded to 96-well GravityPLUS hanging drop plates (InSphero) in sEV- or DPBScontaining media $(5,000$ cell/ $40 \mu 1 /$ well $)$. Microtissue generation was followed for $72 \mathrm{~h}$ and 
images were acquired in every $24 \mathrm{~h}$ using an Axiovert S100 microscope (Zeiss) equipped by a Nikon D5000 camera. To quantify differences in size and shape between the microtissues, $72 \mathrm{~h}$ images were grey-scaled and analysed by the AnaSP software ${ }^{74}$. Measured parameters of microtissues were the equivalent diameter, major diameter through centroid, minor diameter through centroid, convexity, solidity, sphericity, area, perimeter and volume. The experiment was repeated 3 times.

\subsection{Statistical analysis}

Since the homogeneity of variances assumption of the ANOVA (analysis of variance) had not met with our data, statistical analyses were performed by the Welch's ANOVA test with Tukey's honestly significant difference (HSD) post-hoc test (Alpha=0.05) using a Microsoft Excel add-in, the Real Statistics Resource Pack software (Release 5.4) Copyright (2013-2018) Charles Zaiontz (www.real-statistics.com). Diagrams were prepared in GraphPad Prism 5.03. All average values represent mean $\pm \mathrm{SD}$ and number of asterisk denote minimum statistical significance, i.e. ${ }^{*} \mathrm{p}<0.05,{ }^{*} \mathrm{p}<0.01$ and $* * * \mathrm{p}<0.001$ on figures. Exact $\mathrm{p}$-values are indicated in the text, when it is necessary. 


\section{Results}

In order to study the adaptive responses in the sEV-mediated communication of tumour cells under different microenvironmental conditions, we have investigated stress-induced changes of the 5-8F human NPC- and the B16F1 mouse melanoma cell-derived sEVs.

\subsection{Investigation of the NPC-derived SEVs}

As a first step, untreated 5-8F cell-released sEVs were isolated by differential filtration and ultracentrifugation and characterised by AFM. Then, 5-8F cell cultures were exposed to cytostatic and oxidative stress as they were treated with $0.6 \mu \mathrm{M}$ doxorubicin (Doxo) or $2.5 \mu \mathrm{g} / \mathrm{ml}$ light-induced $\mathrm{Ag}-\mathrm{TiO}_{2}\left(\mathrm{Ag}-\mathrm{TiO}_{2}\right)$, respectively. Untreated cultures were used as a control (Ctrl). After $72 \mathrm{~h}$, sEVs were isolated from the cell culture supernatants, quantified by NTA and subjected to SOLiD sequencing to determine the miRNome of sEV groups. The obtained miRNA data were analysed in silico using the IPA software to reveal functional differences between sEV groups (Fig. 3).

\section{(1) sEV production \\ 2 sEV characterization}

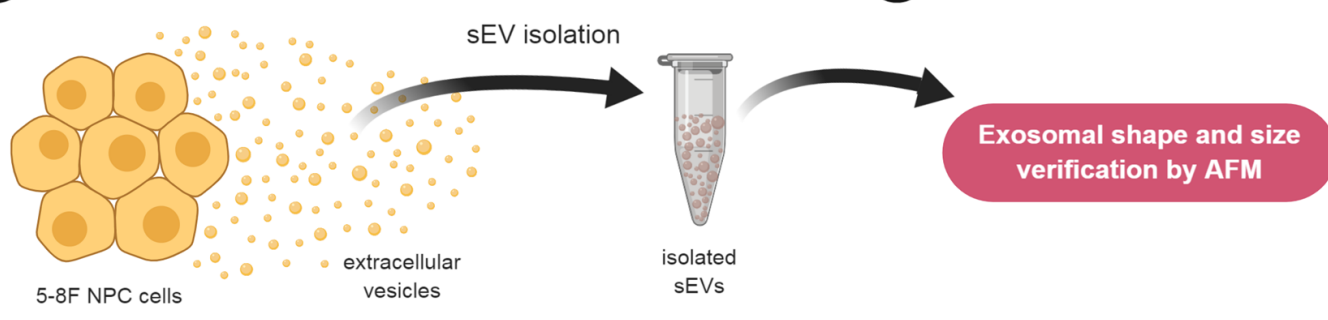

3 Stress exposures

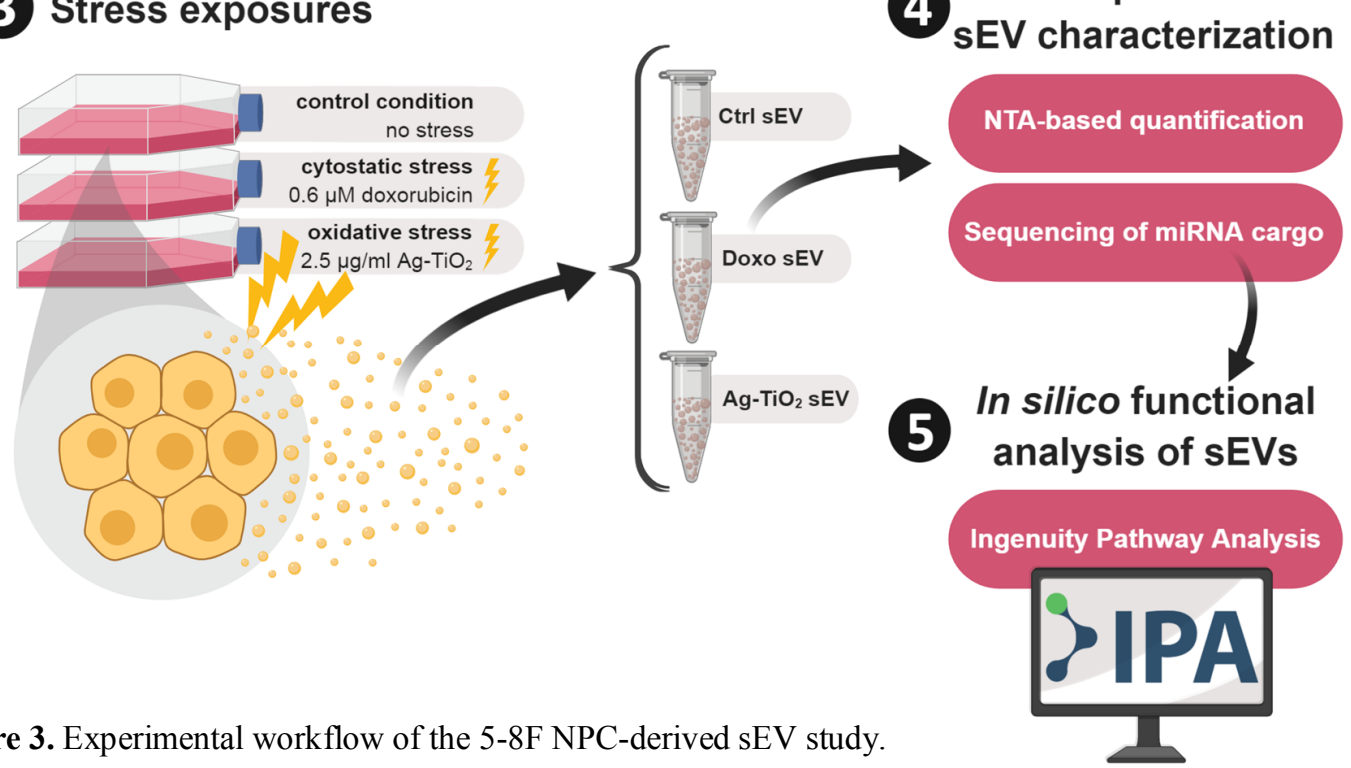

Figure 3. Experimental workflow of the 5-8F NPC-derived sEV study.

\section{Comparative EV characterization}

NTA-based quantification

Sequencing of miRNA cargo In silico functional analysis of sEVs Ingenuity Pathway Analysis IPA 


\subsubsection{NPC vesicle isolates contained exosome-sized sEVs}

Presence of vesicles in the isolates and their exosomal shape and size was verified by AFM. Acquired images showed intact vesicles with a 30-150 nm size range (Fig. 4a-c).

\subsubsection{Descriptive statistics of the NPC-derived SEVs released under different microenvironmental conditions}

\subsubsection{1 $\mathrm{sEV}$ release is elevated under stress conditions}

Using the NTA method by a NanoSight NS500 instrument, quantity of sEVs were determined in the isolates. The number of sEVs was significantly higher in the samples of the cytostatic $\left(2.74 \times 10^{12} \pm 2.78 \times 10^{11}, \mathrm{p}=0.0146\right)$ and oxidative $\left(3.28 \times 10^{12} \pm 5.16 \times 10^{11}, \mathrm{p}=0.0006\right)$ stressexposed cells compared to the Ctrl cultures $\left(1.96 \times 10^{12} \pm 6.94 \times 10^{10}\right)$, suggesting an elevated sEV production under stress conditions (Fig. 4d).

a

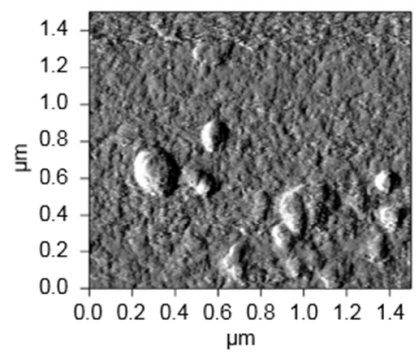

d

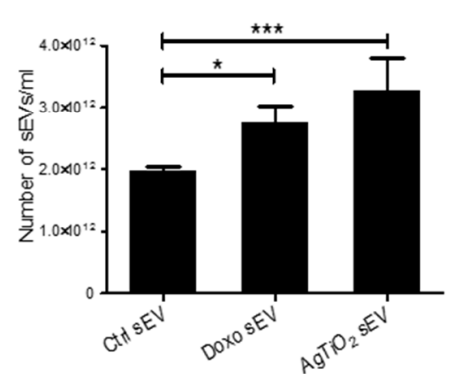

b

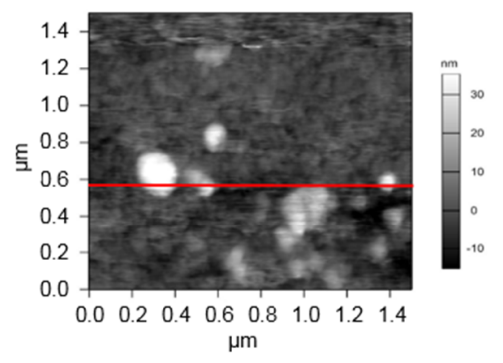

e

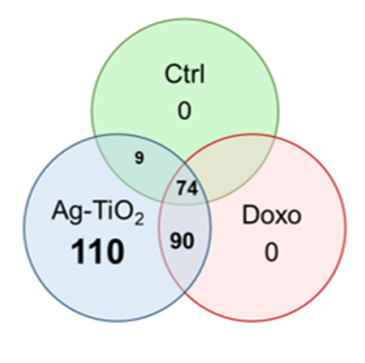

f
C
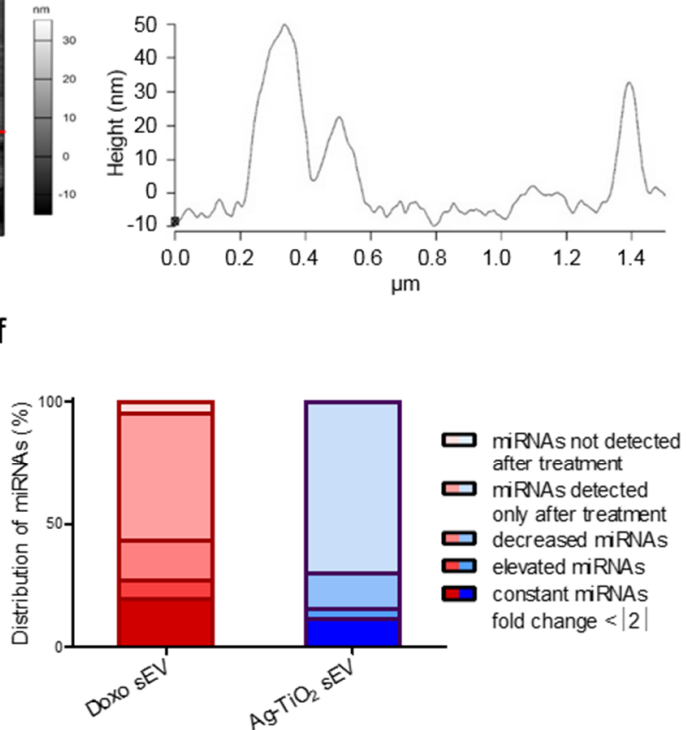

Figure 4. Characterisation of the 5-8F sEVs. (a-c) High-resolution AFM images of intact sEVs. Height (a) and amplitude (b) images $(1.5 \times 1.5 \mu \mathrm{m})$ are shown together with height profiles (c) corresponding to the horizontal line assigned on the amplitude image. (d) Number of vesicles in each sEV group quantified by NTA ( $\mathrm{n}=4$ ), each bar represents mean $+\mathrm{SD} ; * \mathrm{p}<0.05$ and $* * * \mathrm{p}<0.001$ indicate statistical significance. (e-f) Results of the miRNA sequencing. Venn diagram (e) shows the number of common and unique molecules in the sEV groups. Stacked bar graph (f) shows the distribution of sEV miRNAs based on their changes compared to the Ctrl sEV group.

\subsubsection{2 miRNome of $S E V s$ is altered under stress conditions}

In this study, we compared the miRNA content of the sEV groups to reveal the influence of the microenvironment on the molecular pattern and function of these information packages. We found a substantial increase in the miRNA diversity under stress conditions. A total of 283 
miRNAs were identified by SOLiD sequencing with more than ten read counts; $26.15 \%$ (74 of 283) of these miRNAs were detected in each sEV group, 31.80\% (90 of 283) were found in both stress sEV groups (but not in Ctrl sEVs), while 38.87\% (110 of 283) were exclusively detected in the $\mathrm{Ag}-\mathrm{TiO}_{2} \mathrm{sEVs}$ (Fig. 4e). Ctrl and Doxo sEV-specific miRNAs were not found. In other words, the number of delivered miRNA types increased by $2.22 \mathrm{x}$ and $3.82 \mathrm{x}$ in the NPC sEVs under cytostatic and oxidative stress, respectively. Comparing the miRNA levels in stress SEV groups to the miRNA levels in Ctrl sEVs, we found that the proportions of the overrepresented miRNAs (fold change $>2$ ) were $59.69 \%$ and $74.36 \%$ in the Doxo and the $\mathrm{Ag}-\mathrm{TiO}_{2} \mathrm{sEVs}$, respectively. Proportions of the underrepresented miRNAs (fold change $<-2$ ) were $20.92 \%$ and $14.42 \%$ in the Doxo and the $\mathrm{Ag}-\mathrm{TiO}_{2}$ sEVs, respectively (Fig. 4f).

\subsubsection{In silico analysis of functional differences between SEV groups}

Since the exosomal cargo is a complex information package containing a large number and wide variety of molecules, it may act on several biological processes in the recipient cells. Here, we aimed to identify these biological processes even for the normal ( $\mathrm{Ctrl}) 5-8 \mathrm{~F}$ sEVs and also for the stress-exposed cell-derived ones (Doxo, Ag-TiO 2 sEVs). We performed bioinformatics analyses to interpret the biological context of the obtained miRNA data applying the IPA. This software is based on computer algorithms that analyse the functional connectivity of the molecules using the 'Ingenuity Knowledge Base'. For these in silico analyses, we set the confidence level to 'Experimentally observed' that enables literature databased analysis, but not unproven predictions. Phrases between apostrophes are 'IPA-specific terms' throughout the dissertation.

\subsubsection{NPC-derived sEVs have cellular and systemic functions as well}

Using the 'Core analysis' feature in the IPA, we found the same 'Top 5 molecular and cellular functions' for all three sEV groups with different significance values. These functions are namely the 'Cellular development', 'Cellular growth and proliferation', 'Cellular movement', 'Cell cycle' and 'Cell death and survival' (Fig. 5a).

Performing 'Comparison analysis' in the IPA, we built a heatmap of the NPC-related 'Biofunctions', significantly influenced by any sEV group $(\mathrm{p} \leq 0.00001)$. This in silico analysis revealed, that the sEVs may play a role not only in intracellular and cellular, but in systemic and immunological processes as well (Fig. 5b). 
a

\begin{tabular}{|l|c|c|c|}
\hline \multirow{2}{*}{$\begin{array}{c}\text { Top } 5 \text { molecular and } \\
\text { cellular functions }\end{array}$} & \multicolumn{3}{|c|}{ P-range } \\
\cline { 2 - 4 } Cell sEV & $\begin{array}{c}\text { Doxo } \\
\text { sEV }\end{array}$ & $\begin{array}{c}\text { Ag-TiO } 2 \\
\text { sEV }\end{array}$ \\
\hline Cellar development & $\begin{array}{r}4.84 \mathrm{E}-02- \\
2.5 \mathrm{E}-09\end{array}$ & $\begin{array}{c}4.84 \mathrm{E}-02- \\
1.63 \mathrm{E}-13\end{array}$ & $\begin{array}{c}5.00 \mathrm{E}-02- \\
7.75 \mathrm{E}-10\end{array}$ \\
\hline $\begin{array}{l}\text { Cellular growth and } \\
\text { proliferation }\end{array}$ & $4.84 \mathrm{E}-02-$ & $4.84 \mathrm{E}-02-$ & $5.00 \mathrm{E}-02-$ \\
\hline Cellular movement & $2.5 \mathrm{E}-09$ & $1.63 \mathrm{E}-13$ & $7.75 \mathrm{E}-10$ \\
\hline Cell cycle & $4.51 \mathrm{E}-02-$ & $4.39 \mathrm{E}-02-$ & $3.68 \mathrm{E}-02-$ \\
& $3.70 \mathrm{E}-05$ & $2.17 \mathrm{E}-08$ & $1.18 \mathrm{E}-08$ \\
\hline Cell death and survival & $4.78 \mathrm{E}-02-$ & $4.61 \mathrm{E}-02-$ & $4.45 \mathrm{E}-02-$ \\
& $5.62 \mathrm{E}-05$ & $8.53 \mathrm{E}-05$ & $2.56 \mathrm{E}-04$ \\
\hline
\end{tabular}

Figure 5. Biological functions of the 5-8F-derived $\mathrm{sEV}$ miRNAs based on in silico analysis. (a) Top 5 molecular and cellular functions influenced by the sEV. (b) Heatmap of the significantly affected 'Biofunctions' by all of the three $\mathrm{sEV}$ groups $(\mathrm{p} \leq 0.00001)$. b

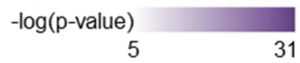

Hypopharyngeal squamous cell carcinoma

Inflammation of organ

Early stage solid tumor

Inflammation of absolute anatomical region

Invasive carcinoma

Pharyngeal carcinoma

Primary solid tumor

Inflammation of body cavity

Fibrosis

Primary tumor

Cell proliferation of tumor cell lines

Advanced malignant solid tumor

Metastatic solid tumor

Stage I-II cancer

Advanced malignant tumor

Secondary tumor

Invasion of tumor cell lines

Migration of tumor cell lines

Cell proliferation of carcinoma cell lines

G1/S phase transition of fibroblast cell lines

Arrest in cell cycle progression of tumor cell lines

Cancer of cells

Invasion of cells

Squamous-cell carcinoma

Cell movement of tumor cell lines

\subsubsection{5-8F sEVs lose their tumour promoting effects, if they are produced in an unfavourable microenvironment}

Using the obtained miRNA data, the 'Grow' tool of IPA enabled to identify the interacting vesicular miRNAs for selected 'Biofunctions'. Then, the 'MAP' feature of IPA predicted their overall regulatory effects, i.e. activation or inhibitory effects for each $\mathrm{sEV}$ group. In this way, we could identify many 'Biofunctions', which can be regulated differently by the sEVs, highlighting the role of the releasing conditions in the vesicular communication of NPC cells.

In general, Ctrl sEVs showed tumour promoting effects; IPA predicted activation of proliferation, viability, migration and EMT, while inhibition of senescence and apoptosis of tumour cells upon exposure to Ctrl sEVs (Fig. 6). However, effects of the stress sEV groups on these 'Biofunctions' decreased or turned into the opposite. For instance, activation of cell viability was predicted by less confidence for the Doxo $\mathrm{sEVs}$ and changed to inhibition for the $\mathrm{Ag}-\mathrm{TiO}_{2} \mathrm{sEV}$. The regulation of migration is predicted to switch to inhibitory upon exposure to both stress-exposed cell-derived sEVs (Fig. 6). These results suggest that miRNA cargo of NPC-derived sEVs loses its ability to promote tumour progression under stress conditions. 
a

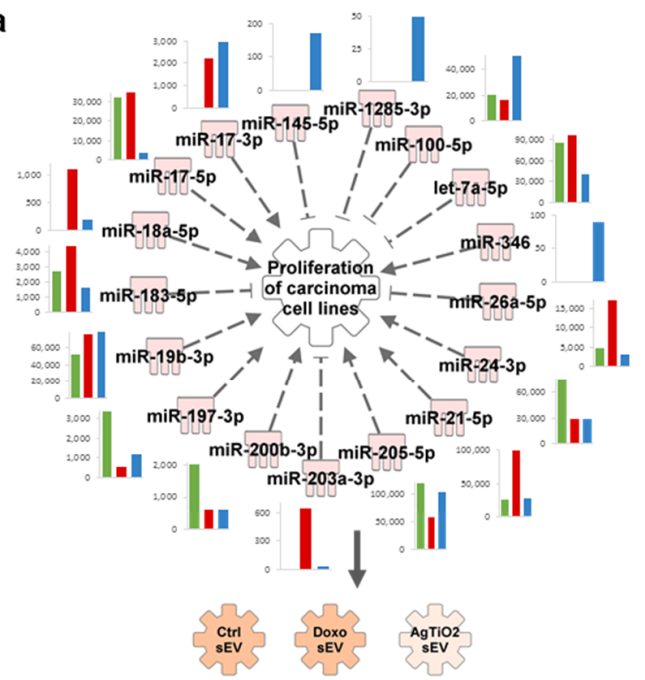

c
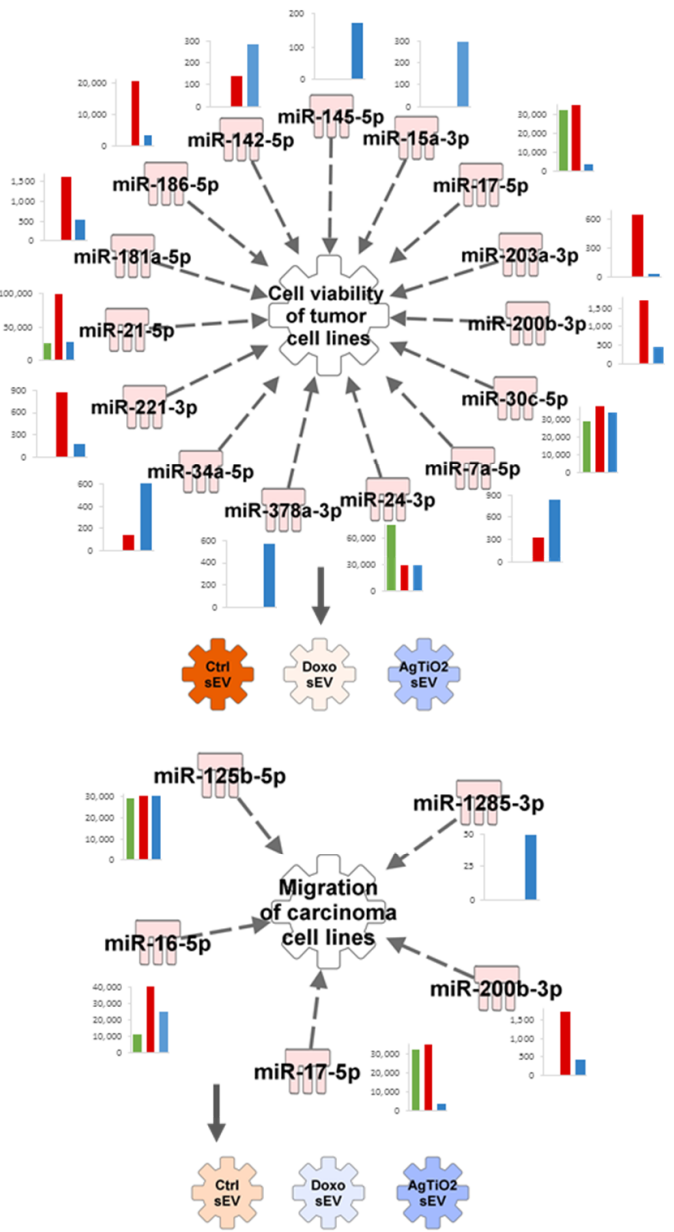
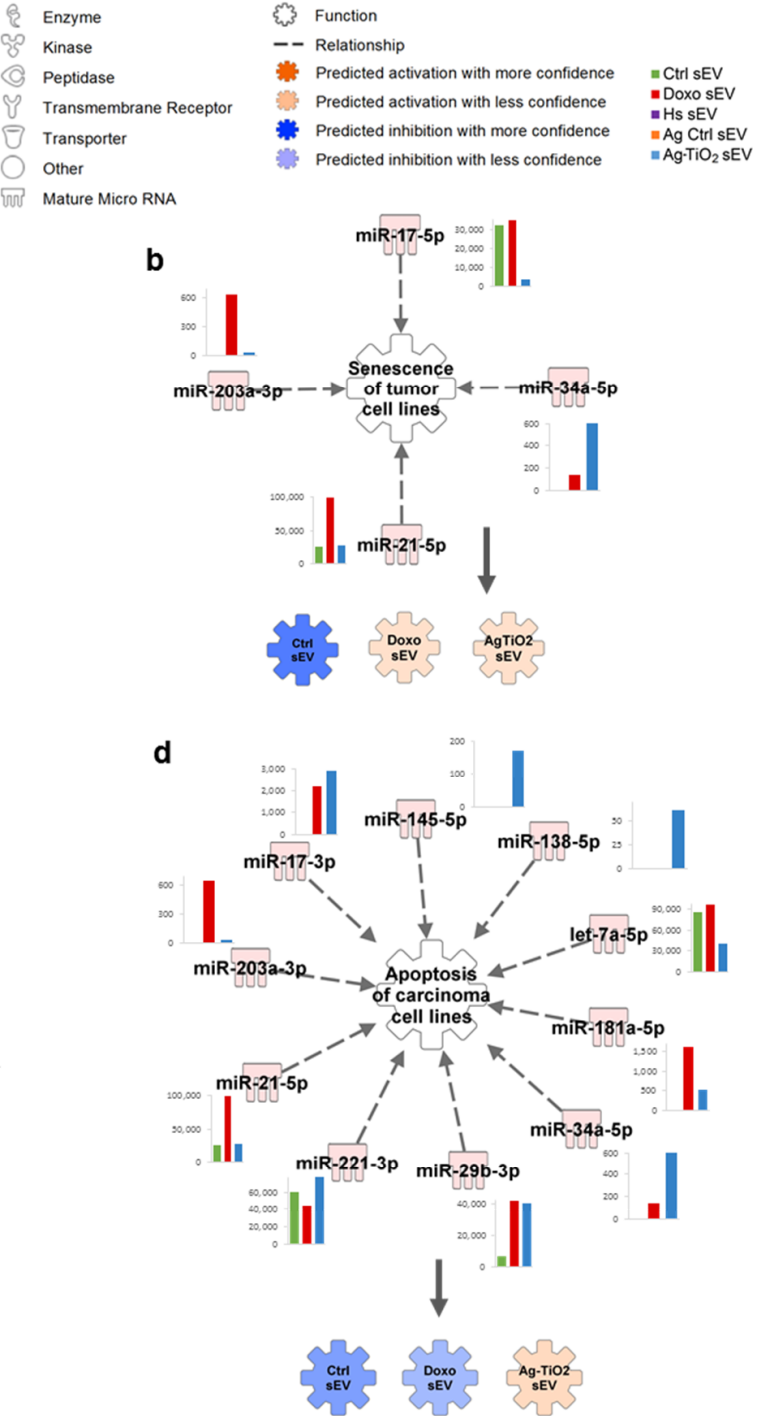

f

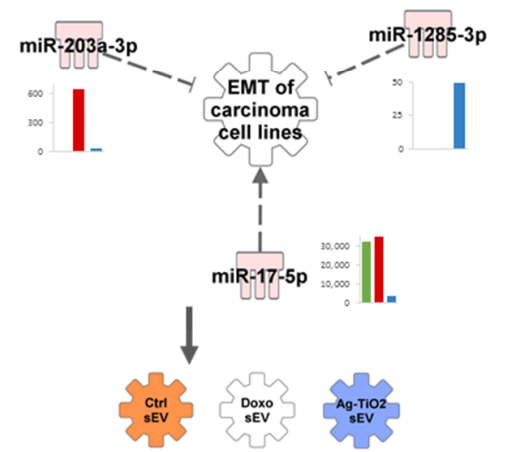

Figure 6. IPA predictions for the regulatory effects of NPC sEV-carried miRNAs on (a) 'Proliferation of carcinoma cell lines', (b) 'Senescence of tumour cell lines', (c) 'Cell viability of tumour cell lines', (d) 'Apoptosis of carcinoma cell lines', (e) 'Migration of carcinoma cell lines' and (f) 'EMT of carcinoma cell lines'. Networks show every upstream regulator miRNAs accompanied by a bar graph, which represents the normalised expression values of the miRNA for each sEV group. Coloured symbols named as the sEV groups display the expected regulation changes of the analysed 'Biofunction' upon exposure to the vesicles. 


\subsection{Investigation of the melanoma-derived $\mathrm{SEVs}$}

In the second part of this project, we aimed to investigate the sEV-mediated intercellular communication of stress-exposed B16F1 melanoma cells. After verification of the exosomal characteristics of the isolated B16F1 sEVs, we investigated their cytostatic, heat and oxidative stress-induced alterations. Melanoma cells were cultured under five different conditions; control cultures $(\mathrm{Ctrl})$ received only culture medium, cytostatic stressed cultures (Doxo) were treated with $0.6 \mu \mathrm{M}$ doxorubicin, heat stressed cultures $(\mathrm{Hs})$ were incubated at $42^{\circ} \mathrm{C}$ for $3 \times 2 \mathrm{~h}$, oxidative stressed cultures $\left(\mathrm{Ag}-\mathrm{TiO}_{2}\right)$ were treated with $2.5 \mu \mathrm{g} / \mathrm{ml}$ light-induced $\mathrm{Ag}-\mathrm{TiO}_{2}$, and

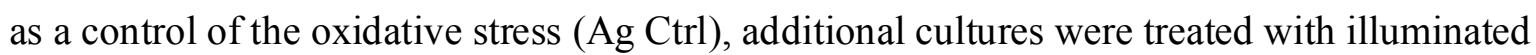
media (Table 1). Then, sEV isolates of the five cell culture groups were analysed by NTA, SOLiD sequencing and LC-MS/MS to determine their vesicle number, miRNome and proteome. Functional differences between sEV groups were predicted first, in silico using the IPA based on the obtained miRNA and protein data, and then verified by in vitro experiments targeting tumour-related cellular functions, such as Ki-67 expression, cell cycle dynamics, migration capacity and microtissue generation of the recipient cells (Fig. 7).

Table 1. Treatment schedule of melanoma cell cultures and the isolated sEV groups.

\begin{tabular}{|c|c|c|c|c|c|}
\hline Conditions & Control 1 & $\begin{array}{c}\text { Cytostatic } \\
\text { stress }\end{array}$ & Heat stress & Control 2 & $\begin{array}{c}\text { Oxidative } \\
\text { stress }\end{array}$ \\
\hline $\begin{array}{c}\text { sEV donor } \\
\text { cell cultures }\end{array}$ & $\mathrm{Ctrl}$ & Doxo & $\mathrm{Hs}$ & $\mathrm{Ag} \mathrm{Ctrl}$ & ${\mathrm{Ag}-\mathrm{TiO}_{2}}^{\text {Treatment }}$ \\
\hline $\begin{array}{c}\text { Released sEV } \\
\text { groups }\end{array}$ & $\mathrm{Ctrl} \mathrm{sEV}$ & Doxo sEV & $\mathrm{Hs} \mathrm{sEV}$ & $\mathrm{Ag} \mathrm{Ctrl} \mathrm{sEV}$ & $\mathrm{Ag}-\mathrm{TiO}_{2} \mathrm{sEV}$ \\
\hline
\end{tabular}




\section{(1) sEV production}

(2) sEV characterisation
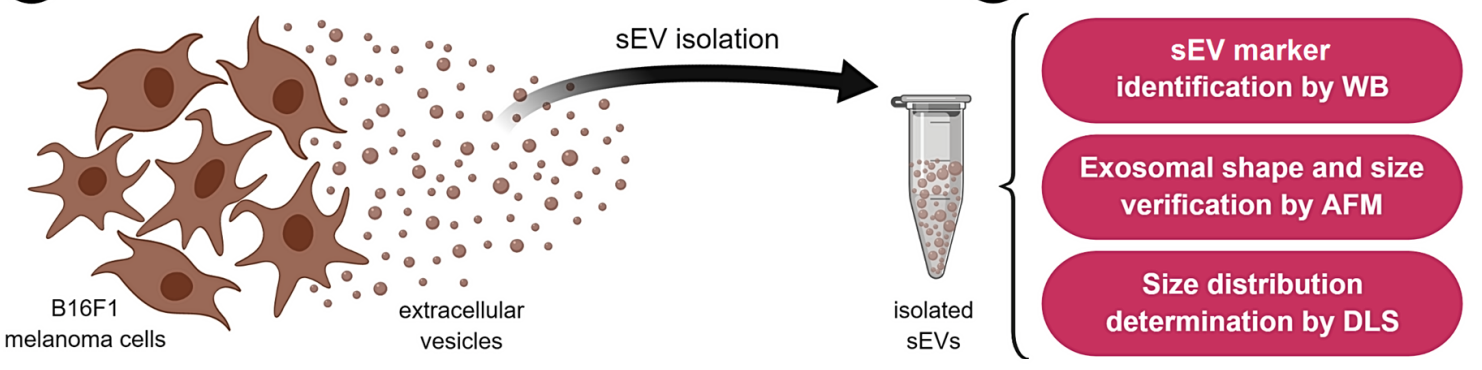

(3) Stress exposures

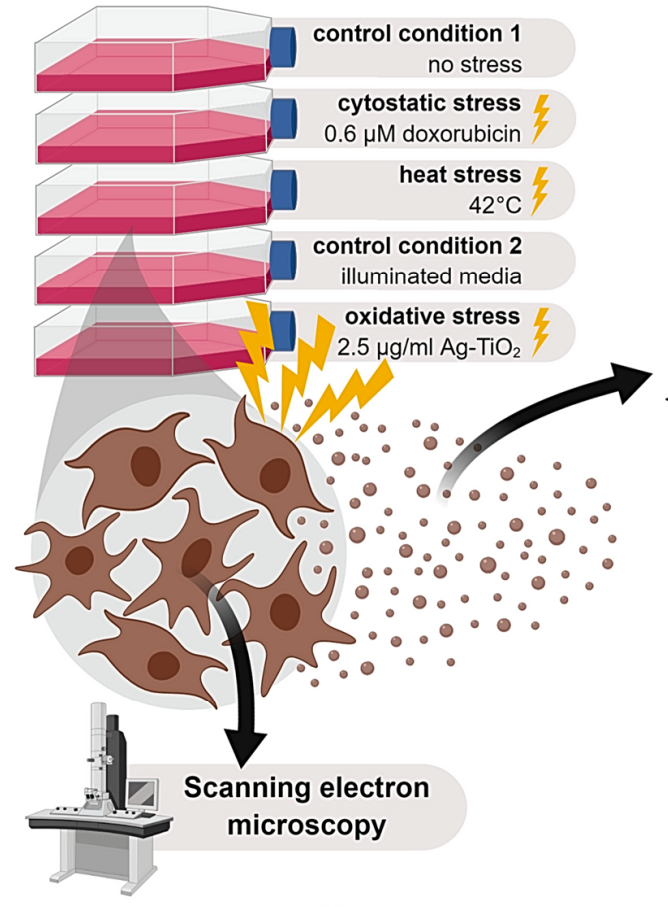

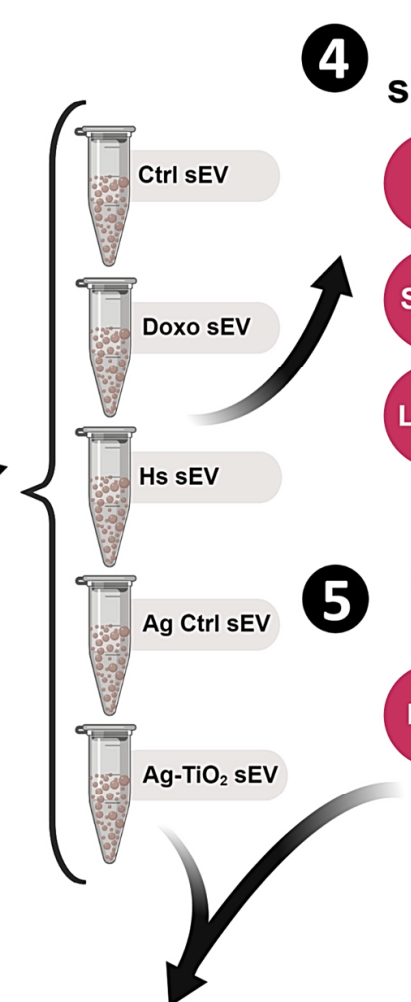

4 Comparative sEV characterisation

NTA-based quantification

Sequencing of miRNA cargo

LC-MS/MS of protein content

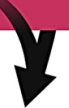

In silico functional analysis of sEVs

Ingenuity Pathway Analysis

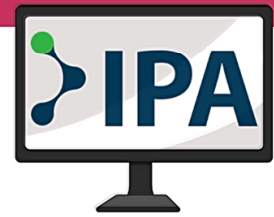

\section{In vitro functional studies of SEVs}

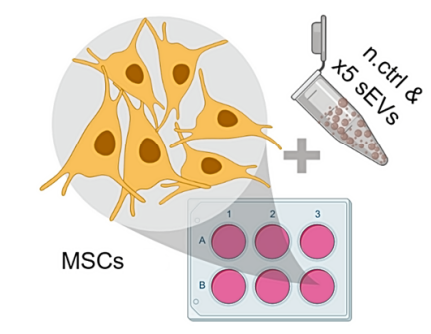

Ki-67 immunocytochemistry with machine learning

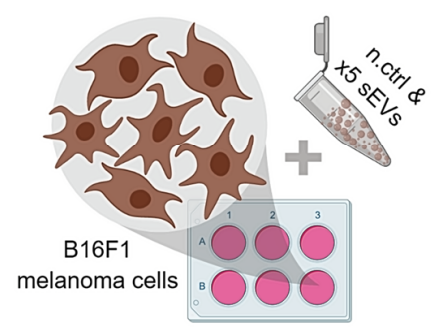

Cell-Clock cell cycle assay with ImageJ analysis

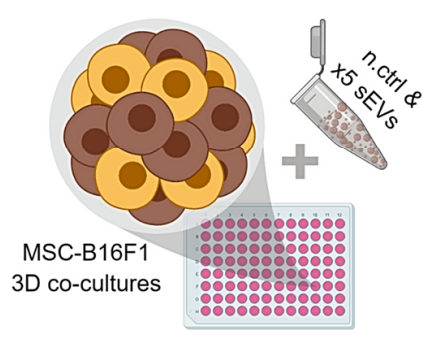

Microtissue generation with AnaSP analysis

Wound healing assay with Image $\mathrm{J}$ analysis

Figure 7. Schematic illustration of the experimental workflow investigating melanoma-derived sEVs. n.ctrl: negative control, $\mathrm{x} 5 \mathrm{sEVs}$ represents the treatments with the $5 \mathrm{sEV}$ groups. 


\subsubsection{Isolated melanoma vesicles fulfill the minimal experimental requirements for small extracellular vesicles (sEVs)}

First, to fulfill the minimal experimental requirements for extracellular vesicles suggested in the MISEV2018 ${ }^{6}$, we characterised the B16F1 cell-derived extracellular vesicles isolated from conditioned media by differential filtration and ultracentrifugation. Presence of the vesicles in the sEV isolates was verified by AFM (Fig. 8a) and size distribution of the isolated vesicle population was described by DLS with a Z-average of $78 \mathrm{~nm}$ (Fig. $8 \mathrm{~b}$ ). EV markers such as CD63, CD9 (transmembrane proteins), HSP70, Alix, TSG101 (cytosolic proteins), but not Calnexin (negative sEV marker) were detected in the vesicle isolates by Western blot (Fig. 8c).

a
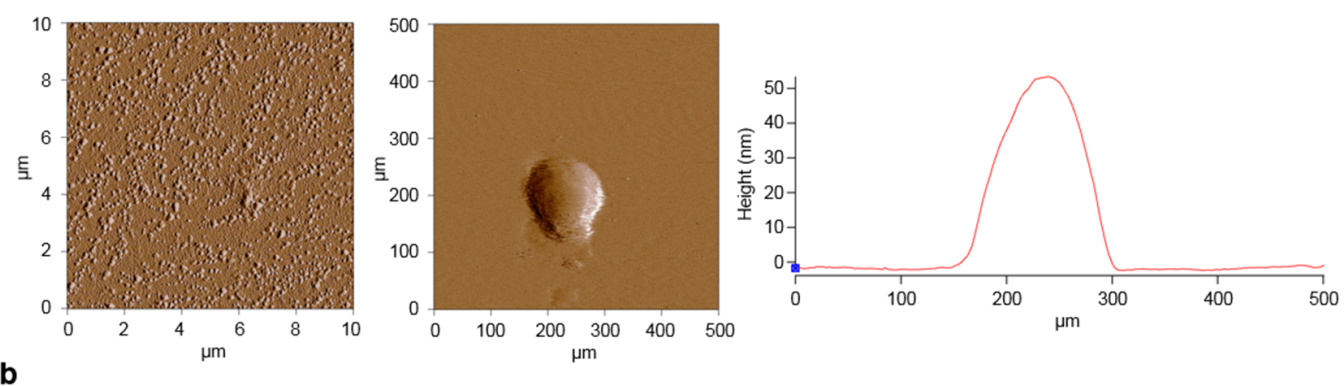

b

Size Distribution by Intensity

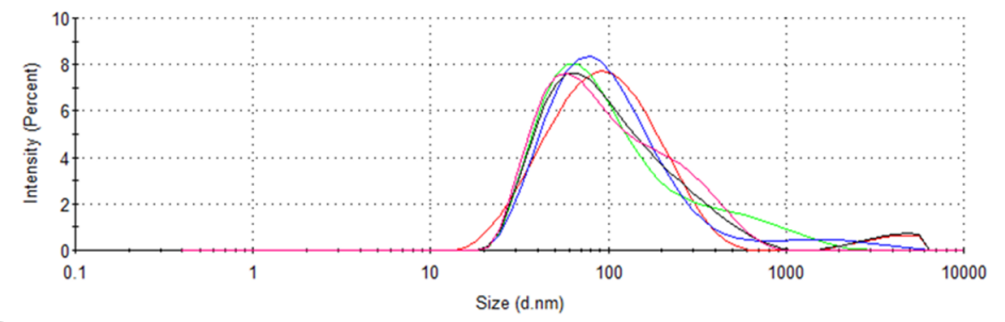

\begin{tabular}{|l|c|}
\hline & $\begin{array}{c}\text { Z-Average } \\
\text { (d.nm) }\end{array}$ \\
\hline Mean & 78.27 \\
\hline SD & 0.7796 \\
\hline
\end{tabular}

c

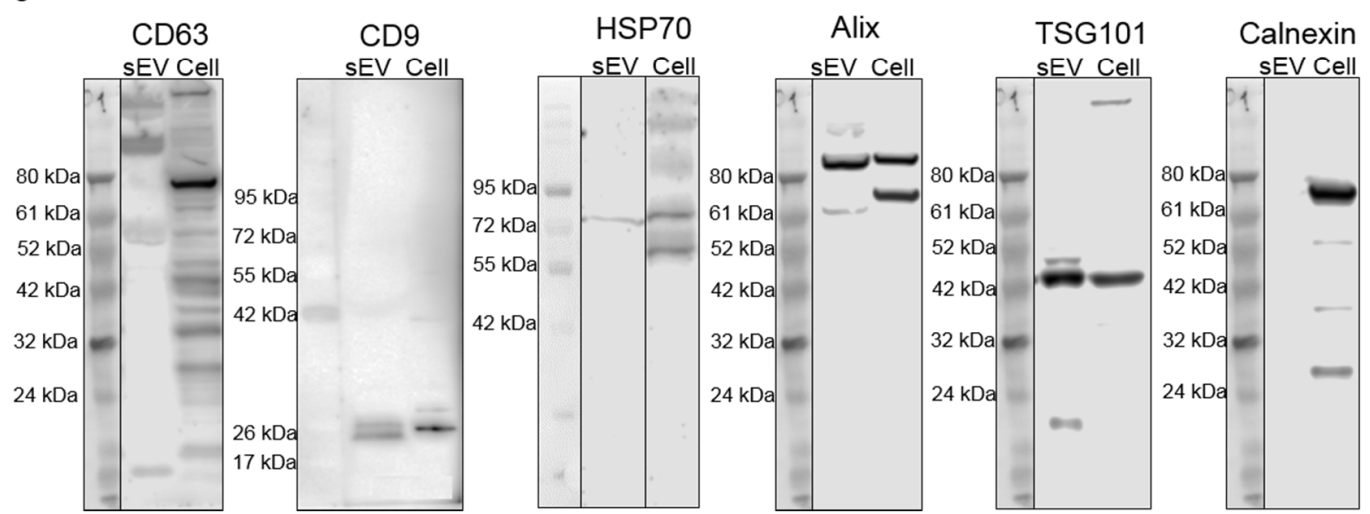

Figure 8. Isolated B16F1 cell-derived vesicles showed exosomal characteristics. (a) Spherical shape and small size of sEVs were verified by AFM. (b) Size distribution of the isolated sEV population was analysed by DLS using a Zetasizer Nano S instrument. (c) EV markers such as CD63 and CD9 (transmembrane proteins), HSP70, Alix and TSG101 (cytosolic proteins), Calnexin (negative sEV marker) were investigated in the vesicle isolates and the donor cell lysates by Western blot. sEV and Cell samples were investigated on the same gels/blots for each marker, however their lines were cropped, shown by black boxes. 


\subsubsection{Descriptive statistics of melanoma $\mathrm{SEVs}$ released under different microenvironmental conditions}

\subsubsection{Vesicle production of melanoma cells is elevated under suboptimal conditions}

SEM revealed spectacular morphological changes of the B16F1 cells in each stressed group (Doxo, $\mathrm{Hs}$ and $\mathrm{Ag}-\mathrm{TiO}_{2}$ ) $24 \mathrm{~h}$ after treatments (Fig. 9a top panels). Taking advantage of the high magnification capacity of SEM, we were able to observe the surface structures of the cells as well (Fig. 8a bottom panels). At a 20,000× magnification, we discovered spherical, exosome-sized vesicles, which were present in higher number on the stressed cells compared to the untreated Ctrl cells ( $\mathrm{p}_{\text {Doxo }}=0.00297, \mathrm{p}_{\mathrm{Hs}}=0.03928, \mathrm{p}_{\mathrm{Ag}_{-}-\mathrm{TiO}_{2}}=\mathrm{n} . \mathrm{s} ., \mathrm{n}=5 ;$ Fig. $9 \mathrm{~b}$ ).
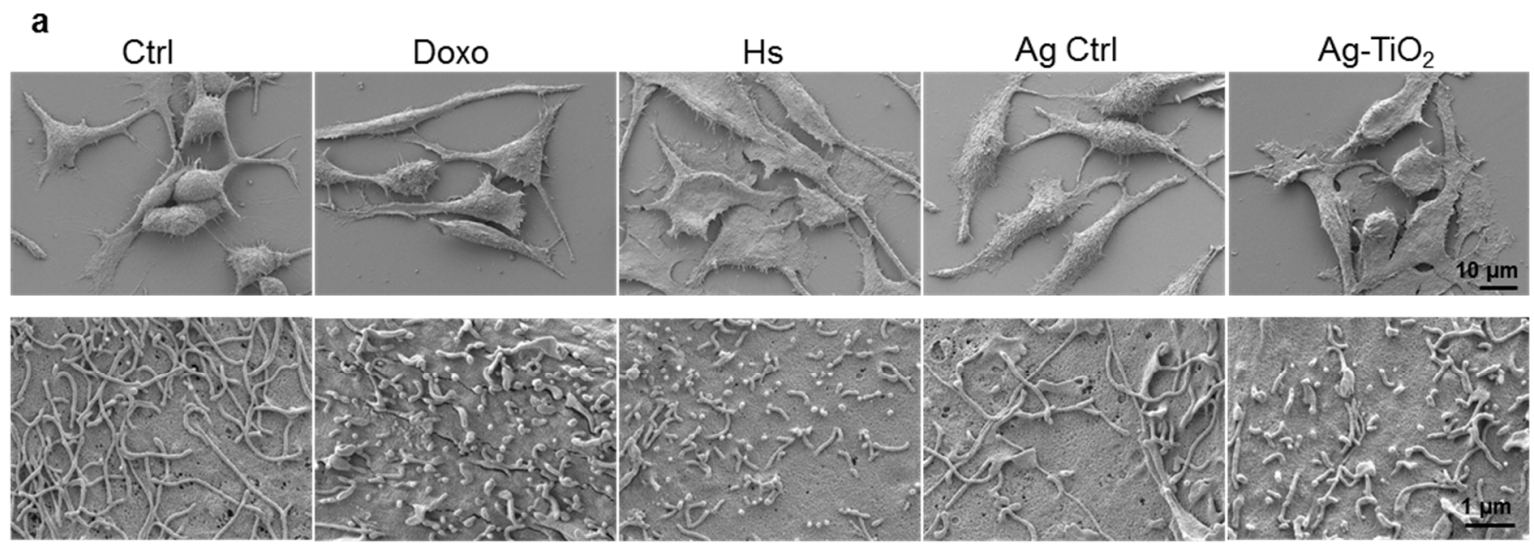

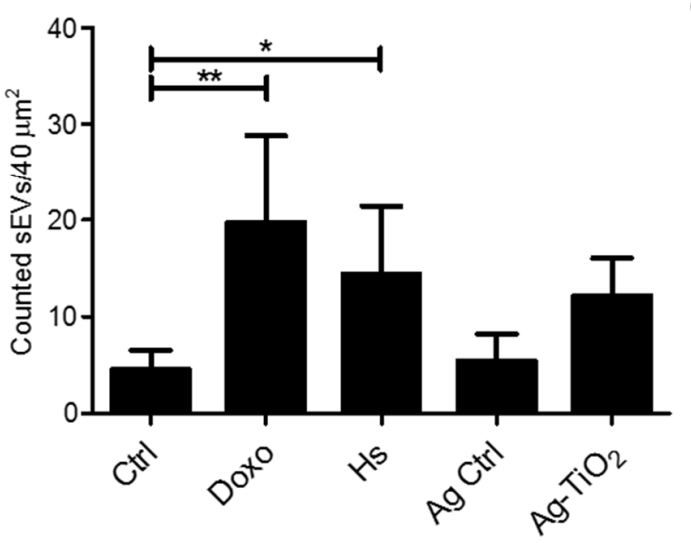

C

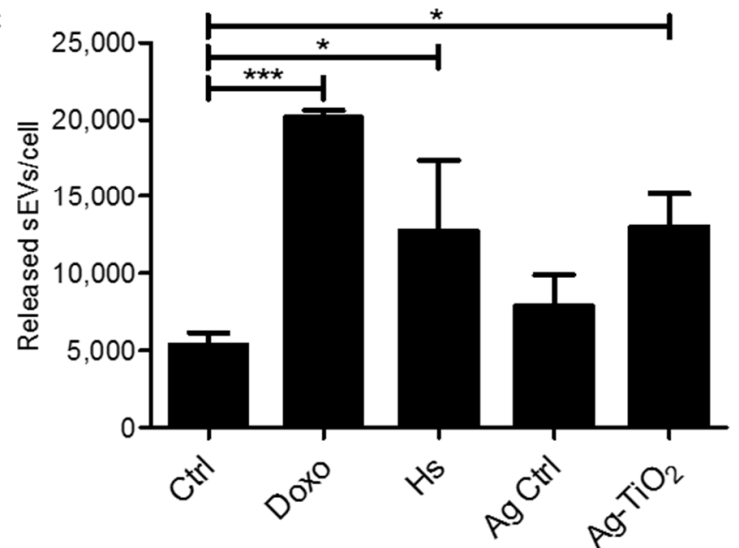

Figure 9. Microenvironmental stress factors resulted in morphological changes and elevated vesicle production of melanoma cells. (a) Scanning electron micrograph of the differently treated melanoma cells. Top row of pictures, taken in 1,500 $\times$ magnification shows the different cell morphology after $24 \mathrm{~h}$ treatments. Bottom row of pictures, taken in 20,000 $\times$ magnification shows the cell surface structures. (b) Counted exosome-sized vesicles on the surface of cells using ImageJ $(n=5)$. (c) Number of released vesicles/cell based on NanoSight measurements $(\mathrm{n}=3)$. Each bar represents mean $+\mathrm{SD} ;{ }^{*} \mathrm{p}<0.05, * * \mathrm{p}<0.01$ and $* * * \mathrm{p}<0.001$ indicate statistical significance.

Isolated sEVs were also quantified by the NTA-based NanoSight analysis. There was a significant increase in vesicle number per cell in the Doxo $\left(20.2 \pm 0.4 \times 10^{3} ; \mathrm{p}=0.00021\right)$ and the 
Hs $\left(12.7 \pm 3.8 \times 10^{3} ; \mathrm{p}=0.03006\right)$ groups compared to the $\mathrm{Ctrl}$ one $\left(5.35 \pm 0.7 \times 10^{3}\right)$. The $\mathrm{Ag}_{-} \mathrm{TiO}_{2-}$ treated cultures produced $13 \pm 1.8 \times 10^{3} \mathrm{sEVs} /$ cell, while production of the $\mathrm{Ag} \mathrm{Ctrl}$ group was only $7.9 \pm 1.6 \times 10^{3} \mathrm{sEVs} / \mathrm{cell}(\mathrm{n}=3$, Fig. $9 \mathrm{c})$. At the same time, stress conditions did not affect the size distribution of sEVs.

\subsubsection{Concentration of the encapsulated doxorubicin under cytostatic stress is less than $10 \%$ of median lethal dose}

To reveal, if doxorubicin - used for cytostatic stress in $0.6 \mu \mathrm{M}$ concentration - could be encapsulated into the vesicles, Doxo sEV isolates were analysed by fluorescence spectroscopy. Using the Ctrl sEVs as a background, the calculated doxorubicin concentration of the Doxo sEVs was $14.735 \mathrm{nM}$.

Based on the measurements, the Doxo sEV suspensions - used for treatment of recipient cells - contained only $8 \mathrm{ng} / \mathrm{ml}$ doxorubicin. Therefore, it cannot be excluded that sEVs may transfer doxorubicin to the recipient cells, but the doxorubicin content of Doxo sEVs is less than $10 \%$ of the median lethal dose (LD50=100 $\mathrm{ng} / \mathrm{ml}$ ) for mouse melanoma cells ${ }^{75}$. So, we concluded, that the effect of transferred doxorubicin may be negligible in the recipient cells.

\subsubsection{Encapsulation of $\mathrm{Ag}^{-\mathrm{TiO}_{2}}$ nanoparticles into $\mathrm{sEVs}$ cannot be proven}

To investigate the possibility of $\mathrm{Ag}-\mathrm{TiO}_{2}$ encapsulation into $\mathrm{sEVs}$, we measured the size distribution of the nanoparticles by DLS. The mean particle size was around $255 \mathrm{~nm}$ during the whole studied time interval (Fig. 10a). This means that the initial $\mathrm{Ag}-\mathrm{TiO}_{2}$ photocatalyst particles form aggregates because the primer size of the $\mathrm{Ag}-\mathrm{TiO}_{2}$ particles is around $25 \mathrm{~nm}$ as seen on the TEM image in our previous paper ${ }^{76}$. Thus, DLS measurements did indicate particle aggregation in the culture medium; however, the size of these particle aggregates did not change during the experiments. These results suggest that the $\mathrm{Ag}-\mathrm{TiO}_{2}$ particles cannot be transferred into the recipient cells in this aggregated form and they cannot contaminate the sEV isolates, since particles over $220 \mathrm{~nm}$ were eliminated during the isolation process.

We also tried to detect disaggregated $\mathrm{Ag}-\mathrm{TiO}_{2}$ nanoparticles in the sEV isolates using CL method and TEM. After photoirradiation of the $\mathrm{Ag}-\mathrm{TiO}_{2}$, generated reactive oxygen species (ROS) can be detected by adding luminol, which emits light upon oxidation ${ }^{63}$. We compared the $\mathrm{CL}$ intensity of $\mathrm{Ag}-\mathrm{TiO}_{2} \mathrm{sEV}$ lysates to an $\mathrm{Ag}-\mathrm{TiO}_{2}$ calibration curve prepared in $\mathrm{Ag} \mathrm{Ctrl}$ sEV lysate, but it was under the detection limit $(0.25 \mu \mathrm{g} / \mathrm{ml}$; Fig. 10b). Moreover, on TEM 
images we could not observe any internal structures of the sEVs or contaminating electrondense nanoparticles in the $\mathrm{Ag}-\mathrm{TiO}_{2}$ sEV isolate (Fig. 10c). These results suggest, that melanoma-derived sEVs do not transfer $\mathrm{Ag}-\mathrm{TiO}_{2}$ nanoparticles to the recipient cells.

a

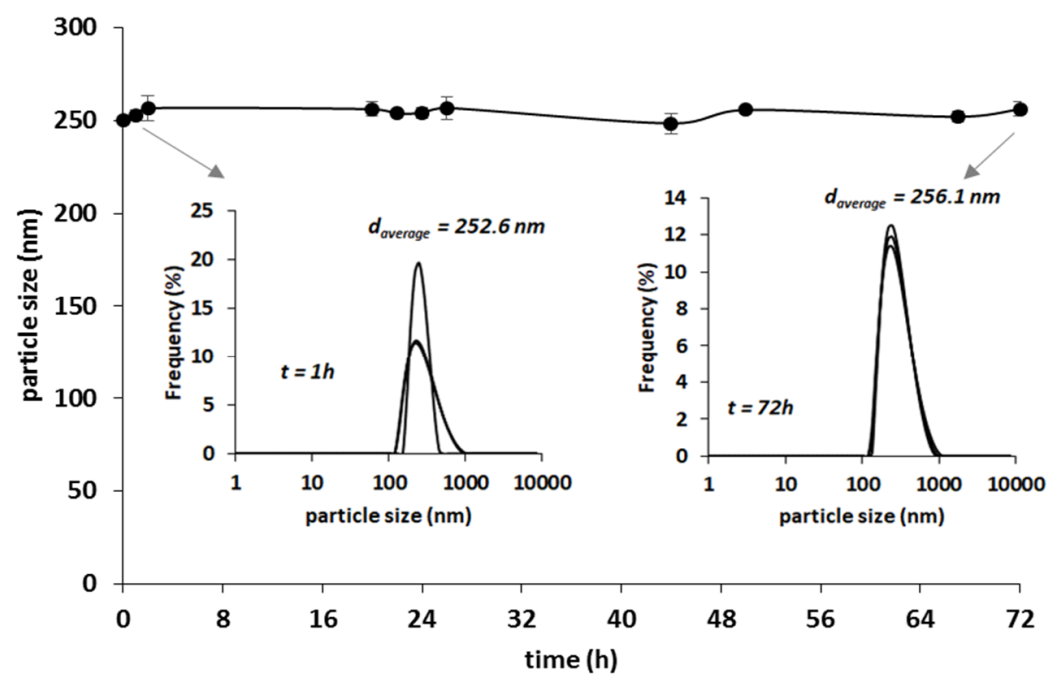

b

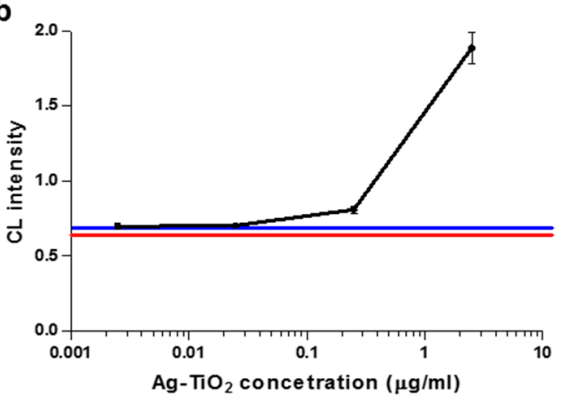

C

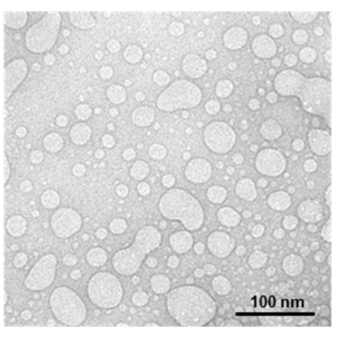

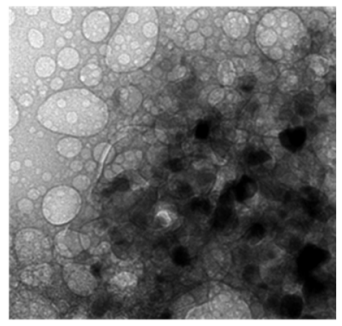

Figure 10. $\mathrm{Ag}-\mathrm{TiO}_{2}$ transfer by sEVs cannot be proven. (a) Results of the DLS measurements. Size distribution of the $\mathrm{Ag}-\mathrm{TiO}_{2}$ nanoparticles was around $255 \mathrm{~nm}$ during the whole time interval of stress exposures. Figure shows the measured average hydrodynamic particle diameter values of $\mathrm{Ag}-\mathrm{TiO}_{2}$ nanoparticles in culture medium, as a function of time. The inserted figures show the size distribution curves measured at $1 \mathrm{~h}$ and $72 \mathrm{~h}$, respectively. (b) Result of the $\mathrm{Ag}-\mathrm{TiO}_{2}$ detection using $\mathrm{CL}$ method. Black line is the $\mathrm{Ag}-\mathrm{TiO}_{2}$ calibration curve (each point represent mean $\pm \mathrm{SD}$ values). Blue and red lines show the mean CL intensities of the Ag Ctrl sEV (background) and $\mathrm{Ag}-\mathrm{TiO}_{2} \mathrm{sEV}$ lysates, respectively. (c) TEM images of $\mathrm{Ag}-\mathrm{TiO}_{2} \mathrm{sEVs}$. Left image shows the $\mathrm{Ag}-\mathrm{TiO}_{2} \mathrm{sEVs}$, which do not contain inner structures, including electrondense $\mathrm{Ag}-\mathrm{TiO}_{2}$ nanoparticles. Right image shows EVs mixed with $\mathrm{Ag}-\mathrm{TiO}_{2}$ nanoparticles as a reference.

\subsubsection{4 miRNome and proteome of the melanoma SEVs strongly depend on the microenvironmental conditions of the donor cells}

To assess the microenvironmental stress-induced alterations of the melanoma sEV cargo, we compared the miRNA and protein patterns of our sEV groups.

A total of 254 miRNAs were identified by SOLiD sequencing with more than ten read counts; $35.04 \%$ ( 89 of 254 ) of these miRNAs were detected in each sEV group, while $6.30 \%$ (16 of 
254), $1.18 \%$ (3 of 254 ) and $0.79 \%$ (2 of 254) were exclusively detected in the $\mathrm{Ag}-\mathrm{TiO}_{2}, \mathrm{Ctrl}$ and Doxo sEVs, respectively. Hs and Ag Ctrl sEV-specific miRNAs were not found (Fig. 11a, left panel). The proportion of the over- and underrepresented miRNAs (fold change $>|2|$ ) under stress conditions was the highest in the $\mathrm{Ag}-\mathrm{TiO}_{2} \mathrm{sEVs}$, where $53.41 \%$ of the detected miRNAs showed more than twofold increase or decrease compared to the miRNA levels in the Ag Ctrl sEVs (Fig. 11a, right panel).

Using LC-MS/MS, a total of 216 proteins were detected with three or more peptides; $59.72 \%$ (129 of 216) of these proteins were common to all sEV groups, and only a few unique proteins were found (Fig. 11b, left panel). Eight Hs sEV-specific proteins were identified, half of them had a chaperone function (Hsp70-binding protein 1 - HSBP1, Serpin family H member 1 SERPINH1, chaperonin containing TCP1 subunit 5 - CCT5, clusterin - CLU). The major impact of heat stress on the protein composition of sEVs was also evidenced by the high proportion of the over- and underrepresented proteins (52.21\%) compared to the Ctrl sEV group. The cytostatic stress also resulted in dramatic changes in the protein content of vesicles. For instance, the proportion of proteins, which decreased below the detectable level was the highest (15.34\%) in the case of Doxo sEVs (Fig. 11b, right panel).

a

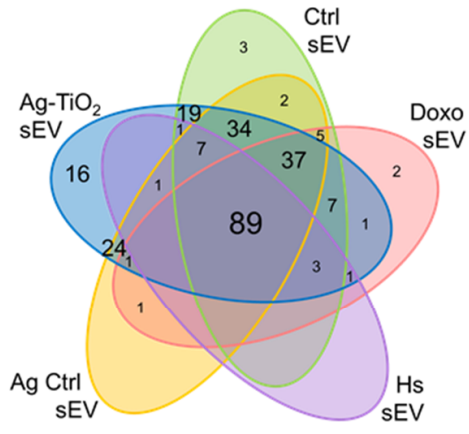

b

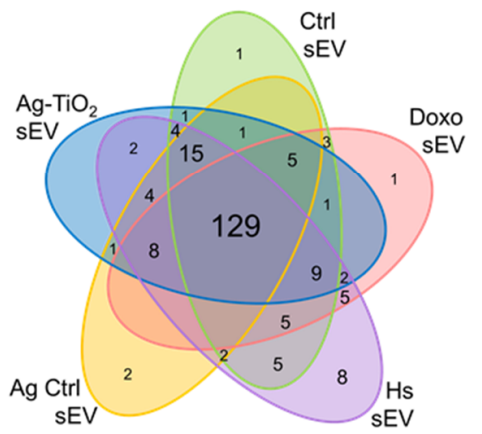

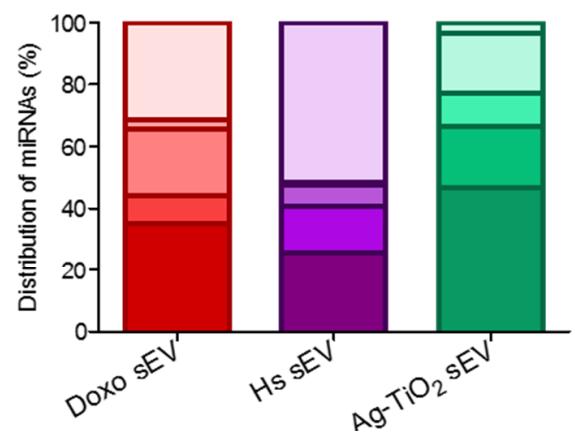

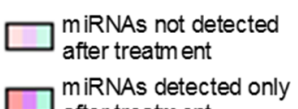

miRNAs detected 0

after treatment

$\square$ dec reased miRNAs

$\square$ elevated miRNAs

constant miRNAs fold change $>|2|$

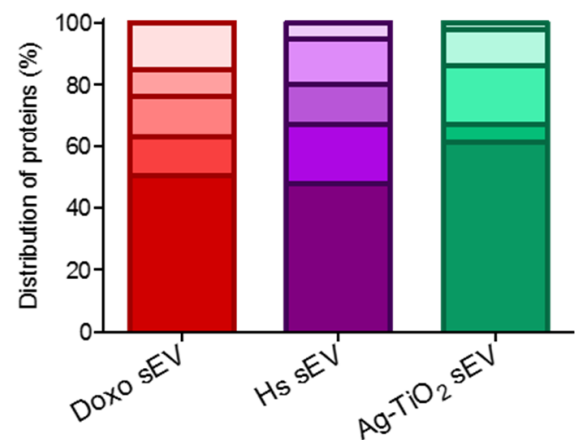

proteins not detected after treatment proteins detected only after treatment decreased proteins

elevated proteins constant proteins,
fold change $>|2|$

Figure 11. Stress factors caused unique molecular patterns of the melanoma-derived sEVs. (a,b) Results of the miRNA sequencing and whole proteome analysis. Venn diagrams show the number of common and unique molecules of the different sEV groups. Stacked bar graphs show the distribution of sEV molecules based on their changes compared to the appropriate control. 
Many of the commonly found proteins have a function in vesicular trafficking, membrane fusion or MVB biology which suggests, they have a role in the exosome biogenesis. There are also melanocyte-specific molecules, such as melanin biosynthesis elements (dopachrome tautomerase - DCT, melan-A (MLANA), premelanosome - PMEL, tyrosinase - TYR or tyrosinase-related protein 1 - TYRP1), and we found several metabolism-, cytoskeletal organisation-, ECM remodelling-related proteins, adhesion molecules, receptors and transporters, which may have a crucial role in tumour progression (Suppl. Fig. S1).

Results of the sequencing and LC-MS/MS were validated on a few sEV molecules using qPCR and Western blot, respectively (Suppl. Fig. S2, S3).

\subsubsection{Comprehensive in silico analysis of functional differences between melanoma sEV groups}

Bioinformatics analysis was performed again to analyse the obtained miRNA and protein data to identify biological processes, which can be influenced by the melanoma sEV groups.

\subsubsection{Melanoma-derived sEVs may affect intracellular, cellular, systemic and immune 'Biofunctions' as well}

Using the IPA 'Core analysis' feature, we found a huge overlap in the 'Top 5 canonical pathways' across the sEV groups. Namely, 'Gycolysis I', 'Gluconeogenesis I', 'Eumelanin Biosynthesis' and 'Phagosome maturation' was listed in all five cases, while 'Inhibition of matrix metalloproteinases' was only found in Ctrl sEVs, and 'EIF2 signalling' was listed in the four other sEV groups (Suppl. Fig. S4a). 'Top 5 molecular and cellular functions' also showed similarities across the sEV groups. 'Cellular development', 'Cellular growth and proliferation', 'Cell death and survival' and 'Cellular movement' were shared between each sEV group, 'Protein synthesis' was listed in Doxo and Hs sEVs and 'Cell-to-cell signalling and interaction' in Ctrl, $\mathrm{Ag} \mathrm{Ctrl} \mathrm{and} \mathrm{Ag}-\mathrm{TiO}_{2}$ ones (Suppl. Fig. S4b).

Based on results of the 'Comparison analysis' in the IPA, we constructed heatmaps of the melanoma-related 'Biofunctions', significantly influenced by any sEV group ( $\mathrm{p} \leq 0.00001)$. In this in silico analysis we were able to identify several intracellular processes, e.g. 'Arrest in G1 phase of tumour cell lines'; cellular processes, e.g. 'Cell movement of melanoma cell lines'; systemic processes, e.g. 'Metastasis of cells' and immunological processes, e.g. 'Activation of leukocytes' as well (Suppl. Fig. S4c-f). 


\subsubsection{In silico predictions-based in vitro analyses of the melanoma $\mathrm{SEV}$-induced cellular responses of tumour matrix cells}

Focusing on the activation and inhibitory effects, the 'Grow' and 'MAP' features of IPA enabled to identify many 'Biofunctions', which can be regulated differently by the sEVs. The in silico predicted $\mathrm{sEV}$-induced cell responses were analysed experimentally by in vitro methods. Proliferation, cell cycle dynamics, migration capacity and microtissue generation of the sEV recipient cells were investigated by Ki-67 immunocytochemistry, Cell-Clock cell cycle assay, wound healing assay and hanging drop technology, respectively.

\subsubsection{Ag-TiO 2 SEVs facilitate proliferation of MSCs}

The in silico analyses predicted activation of Ki-67 expression for the Ctrl, $\mathrm{Hs}$ and $\mathrm{Ag}-\mathrm{TiO}_{2}$ sEVs and activation of 'Proliferation of stem cells' for each sEV group. These predictions suggest that after internalisation by stem cells three of the investigated SEV groups may induce Ki-67 upregulation and each of them can promote cell divisions, if the delivered vesicular cargo is active in the recipient cells (Fig. 12). According to the IPA analyses the key B16F1 vesicular regulator of the Ki-67 expression may be the aldo-keto reductase family 1 member B1 (AKR1B1). It has recently been shown that inhibition of AKR1B1 prevented proliferation and expression of Ki-67 in the human umbilical vein endothelial cells ${ }^{77}$. 'Proliferation of stem cells' could be enhanced for instance by fibronectin (FN1), which was observed by Sharma et al. to increase the growth of embryonic stem cells ${ }^{78}$ (Fig. 12).

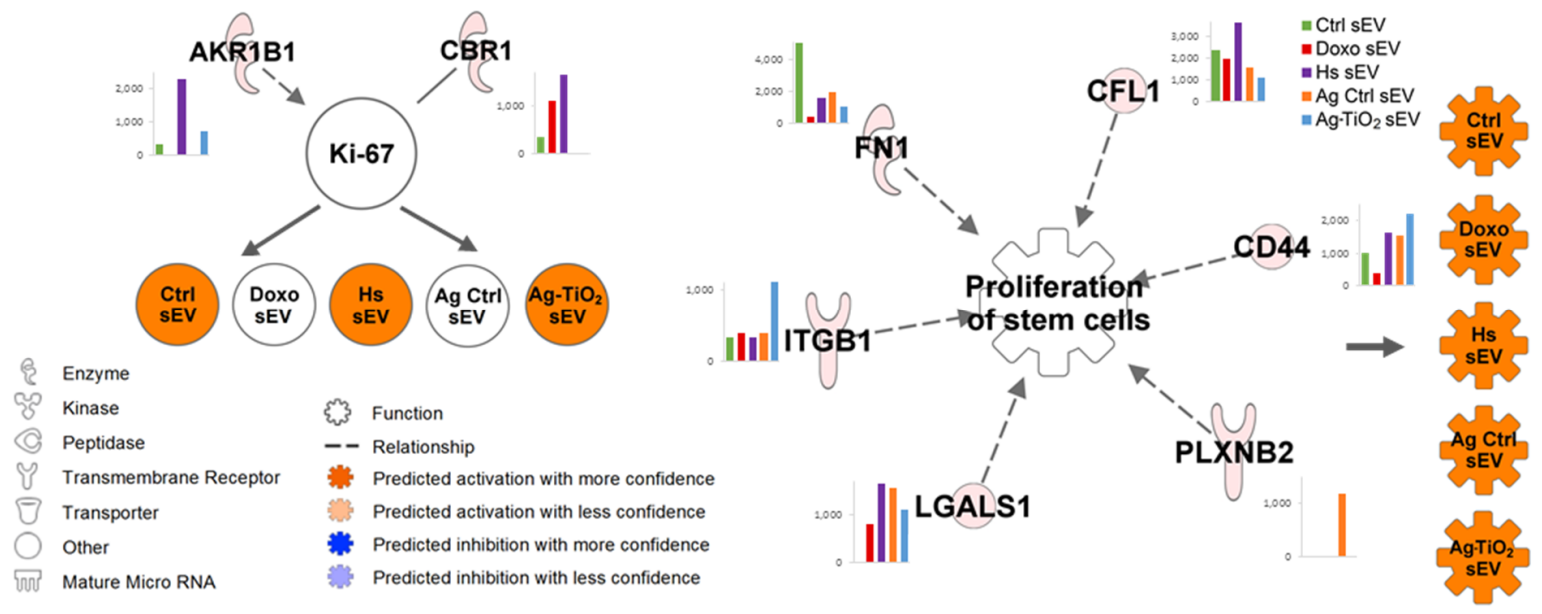

Figure 12. IPA predictions for the regulatory effects of sEV molecules on the Ki-67 expression and 'Proliferation of stem cells'. Networks show every upstream regulator proteins accompanied by a bar graph, which represents the normalised expression values of the molecule for each sEV group. Coloured and symbols named as the $\mathrm{sEV}$ groups display the expected regulation changes of the analysed 'Molecule' and 'Biofunction' upon exposure to the vesicles. 
To test the in silico predicted differences in the Ki-67 regulation across SEV groups, we treated MSC cultures with $200 \mu \mathrm{g} / \mathrm{ml} \mathrm{sEV}$ suspensions or fresh media as a negative control. After $24 \mathrm{~h}$ or $72 \mathrm{~h}$ of vesicle exposures, the Ki-67 expression was investigated by immunocytochemistry. For the quantitative evaluation of the experiment, the Operetta high-content imaging system and an image analysis and machine learning software (SCT Analyzer 1.0 $)^{73}$ was applied, which enabled to analyse almost $1.6 \times 10^{5}$ cells within a few hours. Our computer-assisted image analysis pipeline was comprised of cell segmentation, feature extraction and machine learning modules, where we had a training set with two classes for the Ki-67 positive and the negative cells. Compared to the negative control group, $\mathrm{Ag}-\mathrm{TiO}_{2} \mathrm{sEVs}$ significantly increased the proportion of the Ki-67 positive cells after $72 \mathrm{~h}$ ( $\mathrm{p}=0.03572$, n=4; Fig. 13a-b).

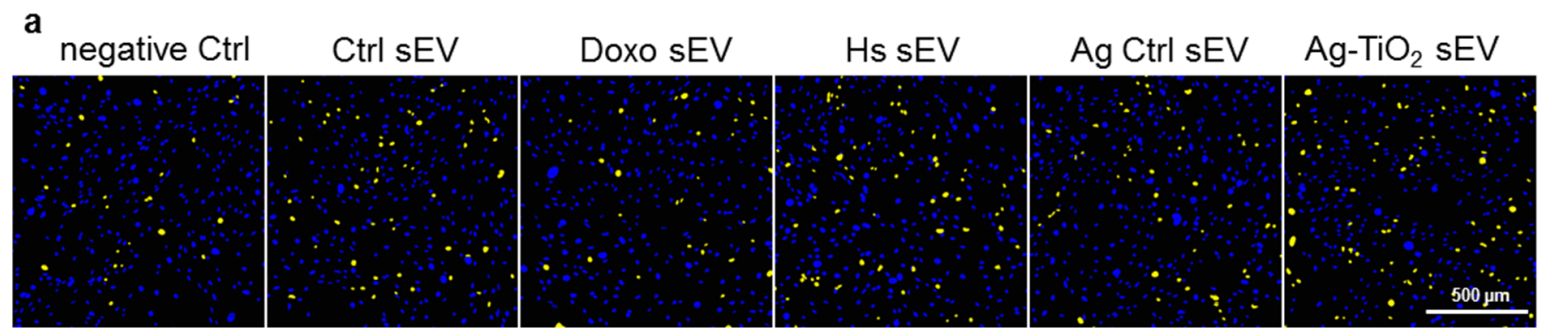

b
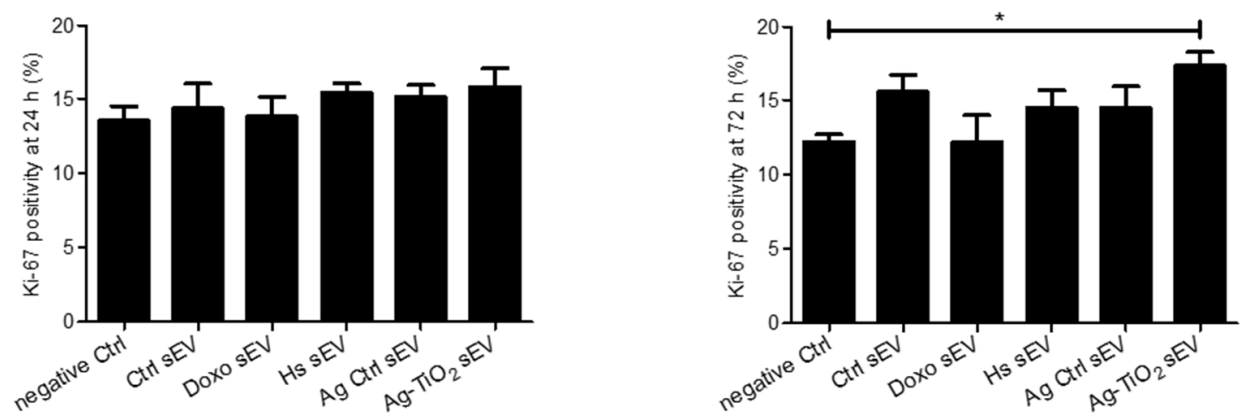

c
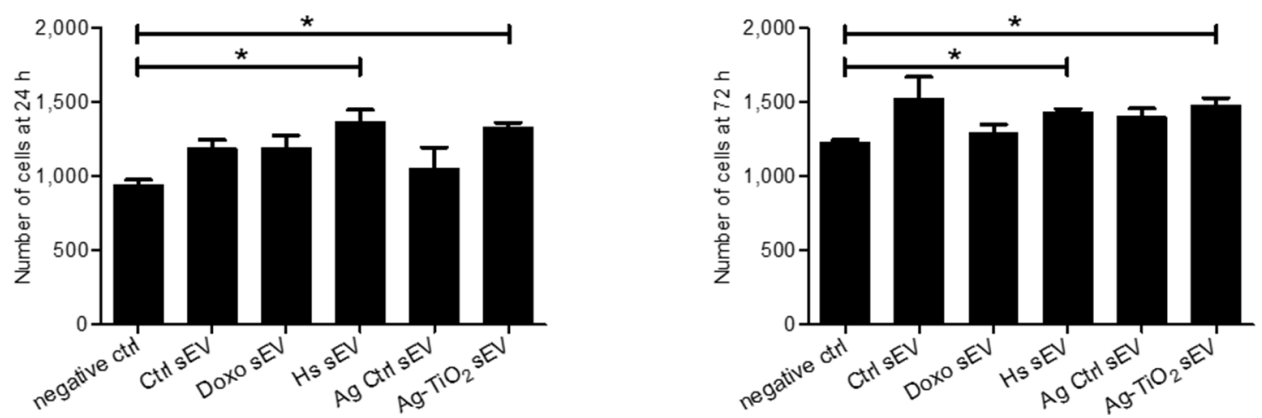

Figure 13 Cell division-related properties of the SEV-exposed MSCs. (a) Images are representatives of the classified ones resulted by the Ki-67 immunocytochemistry and machine learning. Yellow and blue dots show the Ki-67 positive and negative nuclei, respectively. (b) Bar graphs show percentages of the Ki-67 positive cells $24 \mathrm{~h}$ (left panel) and $72 \mathrm{~h}$ (right panel) after sEV exposures. (c) Cell numbers of the sEV-exposed cells after $24 \mathrm{~h}$ (left panel) and $72 \mathrm{~h}$ (right panel) incubation time. Bar graphs represent mean+SD values (n=3), ${ }^{*} \mathrm{p}<0.05$ indicates statistical significance. 
Proliferation of MSCs was also tested by direct cell counting, where all of the cells in the sEVexposed cell cultures were counted using DAPI staining, imaging and machine learning. Results showed increased proliferation of cells upon exposure to $\mathrm{Hs}$ and $\mathrm{Ag}-\mathrm{TiO}_{2} \mathrm{sEVs}$ as early as $24 \mathrm{~h}$, but different sEVs had distinct influence on this cell function (Fig. 13c).

Our in vitro results suggest that melanoma sEVs released under different microenvironmental conditions may have distinct effects on stem cell proliferation. However, beside the IPA predicted interactions, additional molecules and factors, such as the encapsulated doxorubicin, may also be involved in this process.

\subsubsection{Doxo and Ctrl sEVs affect the cell cycle of tumour cells}

IPA analyses predicted inhibition of ' $\mathrm{G} 1$ phase of tumour cell lines' and 'G1/S phase transition of tumour cell lines' upon exposure to Ctrl, Doxo and Hs sEVs (Fig. 14). In other words, the molecular content of these vesicles may arrest the recipient tumour cells in the G1 phase.
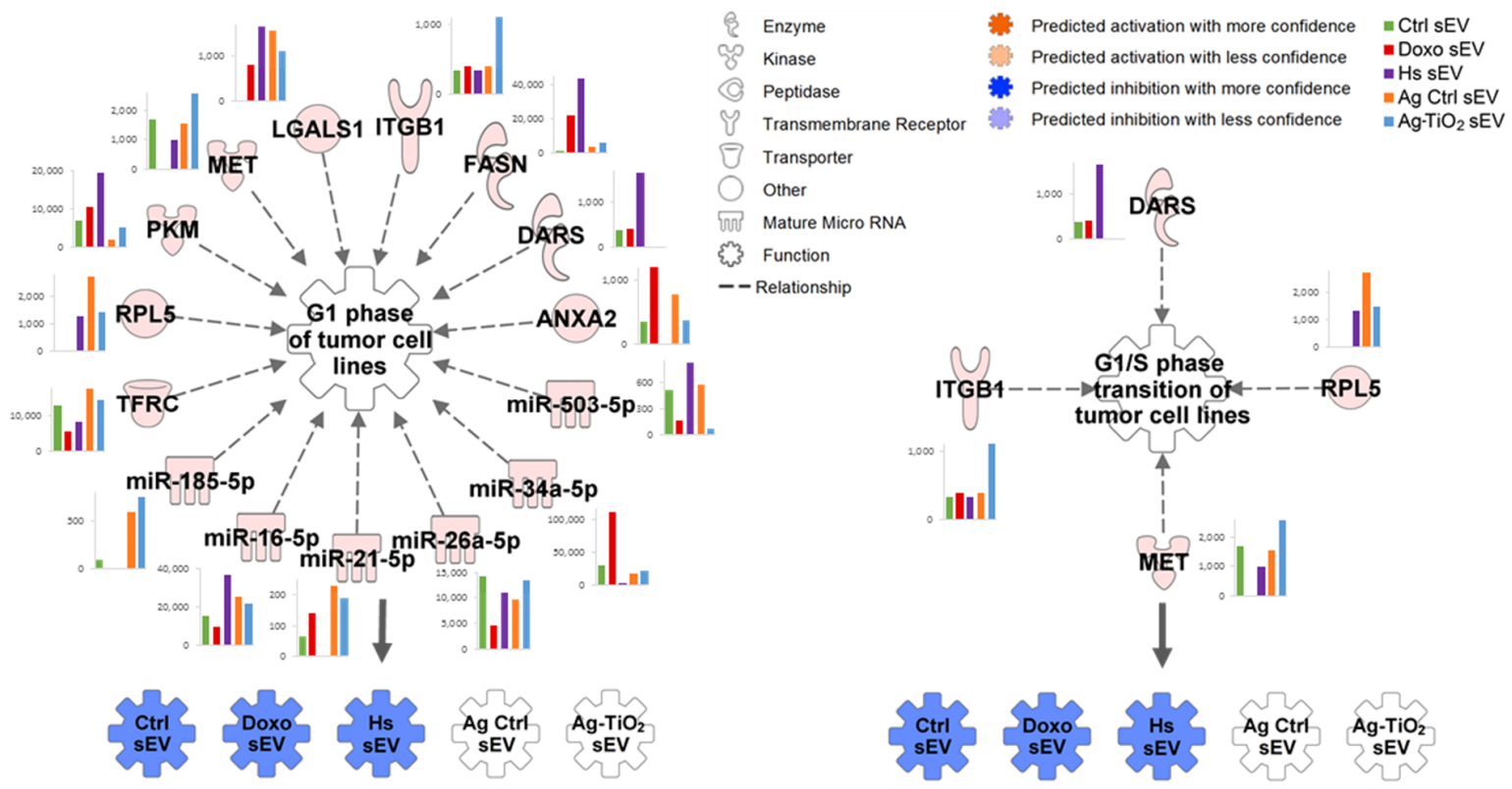

Figure 14. IPA predictions for the regulatory effects of sEV molecules on the 'G1 phase of tumour cell lines' and 'G1/S phase transition of tumour cell lines'. Networks show every upstream regulator proteins and miRNAs accompanied by a bar graph, which represents the normalised expression values of the molecule for each sEV group. Coloured 'Biofunctions' upon exposure to the vesicles.

As displayed on Figure 14, IPA found a total of $15 \mathrm{sEV}$ molecules in our B16F1 data, which may influence the G1 phase of tumour cell lines. The key player of their inhibitory effect may be the aspartyl-tRNA synthetase (DARS), since Yamashita et al. ${ }^{79}$ investigated T24 bladder 
carcinoma cells, and showed that DARS cause a retinoblastoma-independent downregulation of cyclin $\mathrm{A}$, which is required for $\mathrm{S}$ phase entry ${ }^{79}$. Another component of the $\mathrm{B} 16 \mathrm{~F} 1$ vesicles, the p53 inducible miR-34a may lead to apoptosis and cell cycle arrest in the G1 phase, thereby suppressing tumour cell proliferation ${ }^{80,81}$.

To test the in silico predicted effects of sEVs on tumour cell cycle, we performed Cell-Clock cell cycle assay on B16F1 melanoma cells exposed to sEVs for $24 \mathrm{~h}, 48 \mathrm{~h}$ and $72 \mathrm{~h}$ along with a DPBS-treated negative control group. This assay utilizes a vital redox dye that changes colour based on the cell cycle phase. It becomes yellow in G1, green in S/G2 and blue in M phase. In the negative Ctrl group, cells were present in the G1, S/G2, and $M$ phases in average proportions of $44.84 \pm 2.68 \%, 47.80 \pm 3.34 \%$ and $7.35 \pm 0.81 \%$, respectively $(n=12)$.

Ctrl and Doxo sEVs led to an increase in the proportion of the yellow, i.e. G1 phase, cells in a time dependent manner. After $72 \mathrm{~h}$, these cells represented $59.20 \pm 4.06 \%$ of the Ctrl sEVexposed cultures $(\mathrm{p}=0.00346, \mathrm{n}=4)$ and $70.32 \pm 7.24 \%$ of Doxo $\mathrm{sEV}$-exposed cultures $(\mathrm{p}=4.28 \mathrm{E}-06, \mathrm{n}=4)$ (Fig. 15). These results confirmed the IPA predicted arrest in the G1 phase by the Ctrl and Doxo, but not by the Hs sEVs, which means, that Ctrl and Doxo sEVs may inhibit the proliferation of tumour cells.

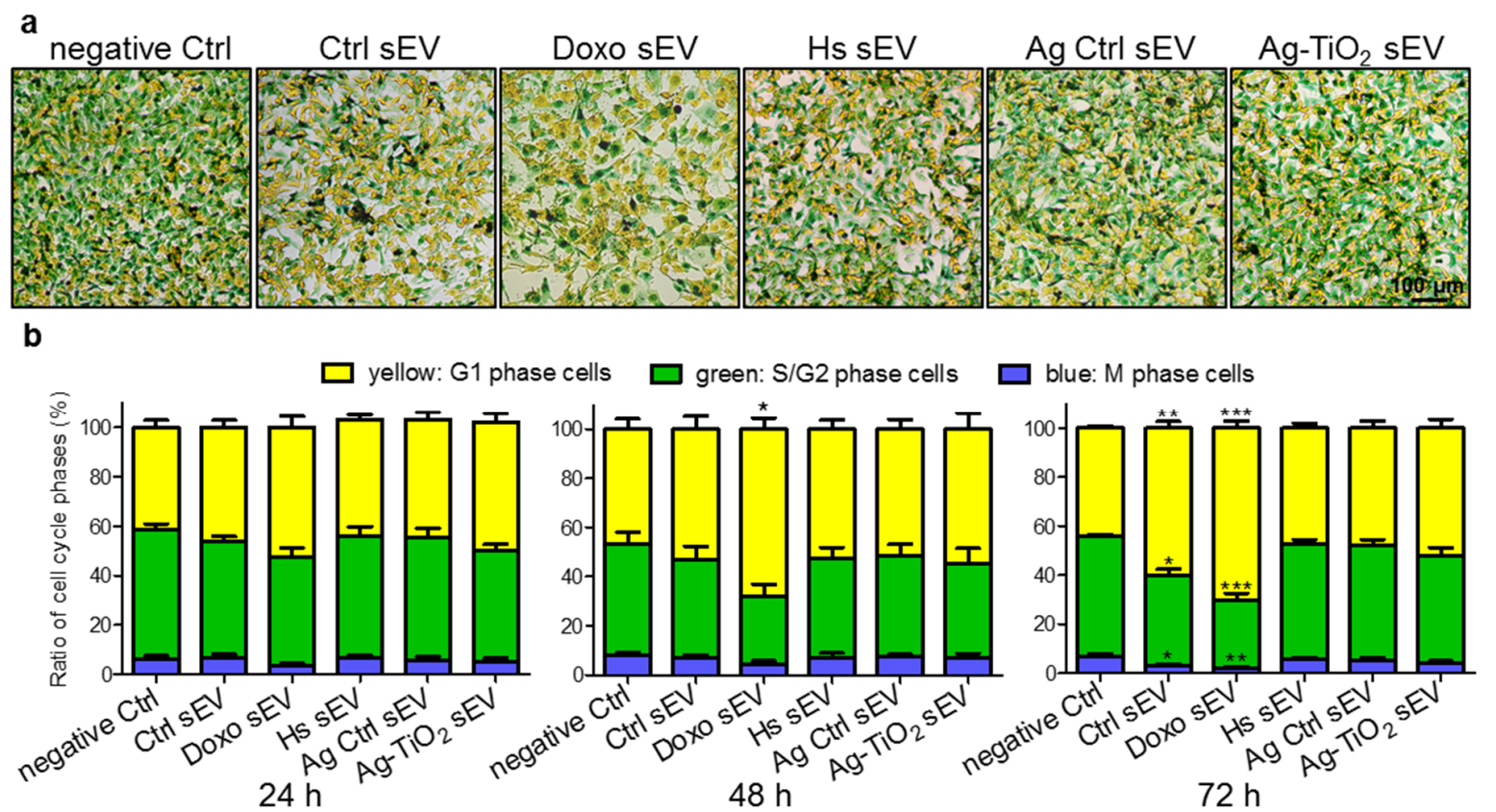

Figure 15. Cell-Clock cell cycle assay of the sEV-exposed B16F1 cell cultures. (a) Representative images of the cell clock dye-labelled cultures. (c) Distribution of the yellow, green and blue cells in the cell cultures, which labels the G1, S/G2 and M phase cells, respectively. Each bar represents mean $+\mathrm{SD}(\mathrm{n}=4),{ }^{*} \mathrm{p}<0.05,{ }^{* *} \mathrm{p}<0.01$ and $* * * \mathrm{p}<0.001$ indicate statistical significance. 


\subsubsection{Migration capacity of melanoma cells is differently altered by the sEVs}

From many cell movement-related 'Biofunctions', which were predicted to be targeted by sEVs (Suppl. Fig 4d), we chose the 'Migration of melanoma cell lines' for further IPA and in vitro investigations. The in silico analyses showed varying sEV effects. More specifically, Doxo and $\mathrm{Ag}-\mathrm{TiO}_{2} \mathrm{sEVs}$ are predicted to facilitate the melanoma cell migration, while the three other ones may inhibit this function (Fig. 16). According to the IPA analyses, negative B16F1 sEV regulators may include the laminin subunit alpha-5 (LAMA5) ${ }^{82}$, peroxiredoxin-2 $(\text { PRDX2) })^{83}$, tyrosinase (TYR) $)^{84}$, let-7a-5 $p^{85}$, miR-125b-5 $p^{86}$ and the miR-34a-5 ${ }^{87}$. Listed positive regulators include the CD44 antigen $(\mathrm{CD} 44)^{88-91}, \mathrm{CD} 81$ antigen $(\mathrm{CD} 81)^{92}$, basigin $(\mathrm{BSG})^{93}$, integrin $\beta-1$ (ITGB1) $)^{94,95}$ and galectin-3-binding protein (LGALS3BP) ${ }^{96}$ (Fig. 16).
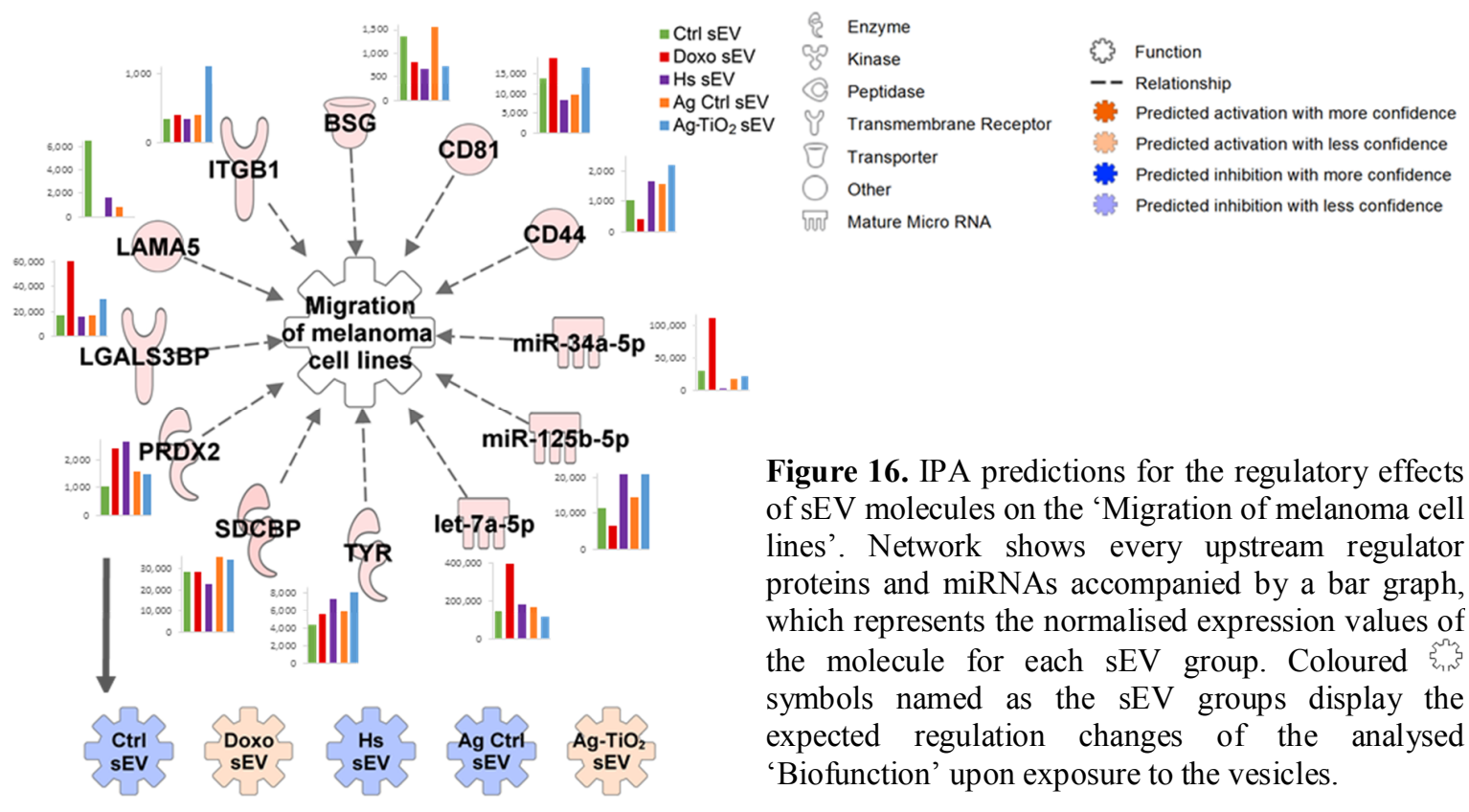

Figure 16. IPA predictions for the regulatory effects of sEV molecules on the 'Migration of melanoma cell lines'. Network shows every upstream regulator proteins and miRNAs accompanied by a bar graph, which represents the normalised expression values of the molecule for each sEV group. Coloured symbols named as the $\mathrm{sEV}$ groups display the expected regulation changes of the analysed 'Biofunction' upon exposure to the vesicles.

Using wound healing assay, we investigated the in silico predicted effects of sEVs on migration of B16F1 cells, which approximated to the IPA-predicted tendency. Migration of cells into the wounded area was slightly decreased in the presence of Ctrl and Ag Ctrl sEVs, compared to migration of the negative Ctrl cells. Acceleration of wound closure was observed in response to Doxo sEVs $(\mathrm{n}=8)$. However, $\mathrm{Hs}$ and $\mathrm{Ag}-\mathrm{TiO}_{2}$ sEVs had no effect on tumour cell migration (Fig. 17). This means that under cytostatic stress, sEVs may convey a message to the neighbouring tumour cells, which enhance their migration. 


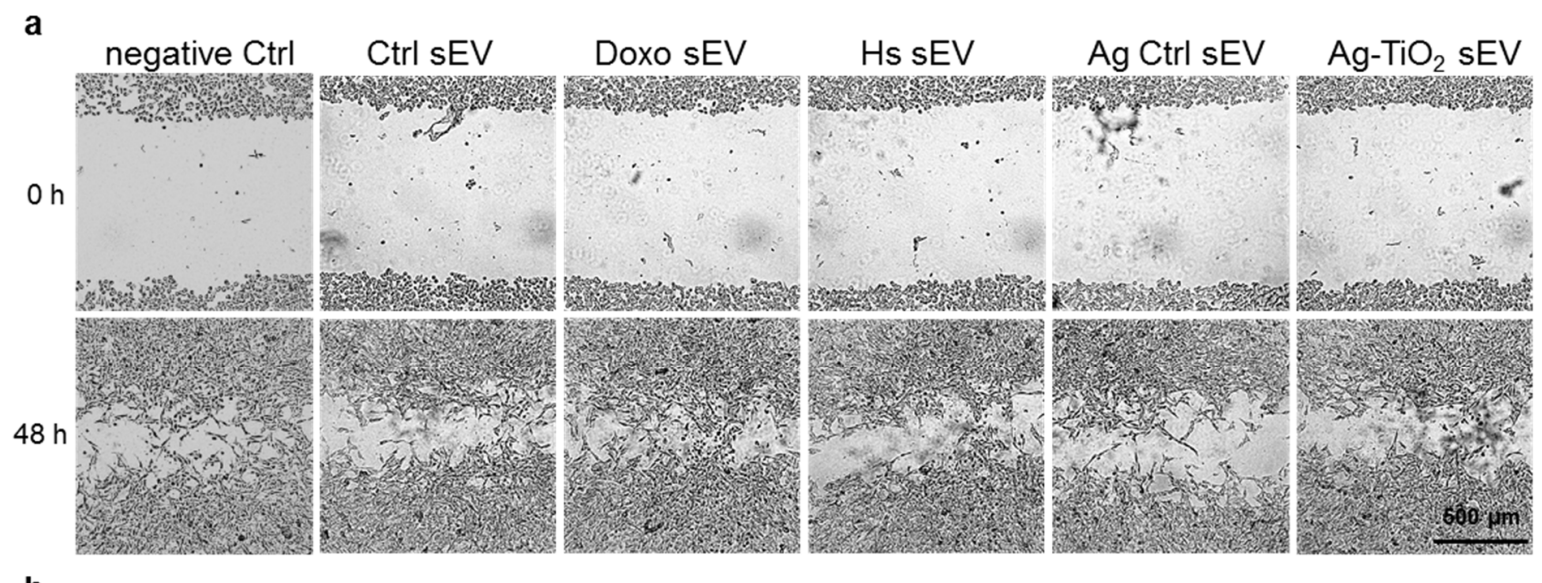

b

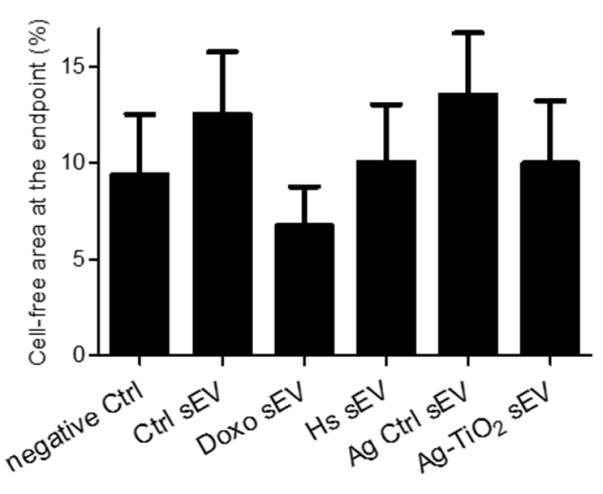

Figure 17. Wound healing assay of the sEV-exposed B16F1 cell cultures. (a) Representative images of the wounds after $48 \mathrm{~h}$ of sEV exposures. (b) Bar graph shows the result of the analysis of wound closures by the ImageJ wound healing tool. It represents mean $+\mathrm{SD}$ values $(\mathrm{n}=8)$.

\subsubsection{Each $\mathrm{sEV}$ group enhances the migration of endothelial cells}

IPA predicted the activation of 'Cell migration of endothelial cells' and 'Cell spreading of endothelial cells' upon exposure to each sEV groups, labelling a large number of potentially contributing sEV molecules (Suppl. Fig. S5). Therefore, we repeated the wound healing assays on bEnd.3 mouse endothelial cells, where we could verify the IPA predictions. However, Ctrl sEVs showed the highest migration enhancing effect (Suppl. Fig. S5) suggesting that released vesicles under normal conditions may promote angiogenesis, but some type of stress conditions may slightly decrease this promoting effects of sEVs.

\subsubsection{Microtissue generation is facilitated independently of the SEV groups}

IPA predicted that each of the five sEV groups could activate many 'Biofunctions' related to the formation of a 3D cell interaction matrix, e.g. 'Aggregation of cells', 'Formation of ECM' (Fig. 18), 'Cell-cell contact' or 'Interaction of tumour cell lines' (Suppl. Fig. S6). The intensity of these activations is variable between sEV groups, for example activation of the 'Aggregation of cells' is predicted to be the strongest upon Doxo and Hs sEV exposures. In silico analyses 
revealed a large number of contributing B16F1 sEV molecules. For instance, it is worth to mention the programmed cell death 6 interacting protein (PDCD6IP, Alix). It has an important role not only in the exosome biogenesis ${ }^{5}$, but Pan et al. demonstrated that an extracellular subpopulation of Alix regulates integrin-mediated cell adhesions and fibronectin matrix assembly ${ }^{97}$.

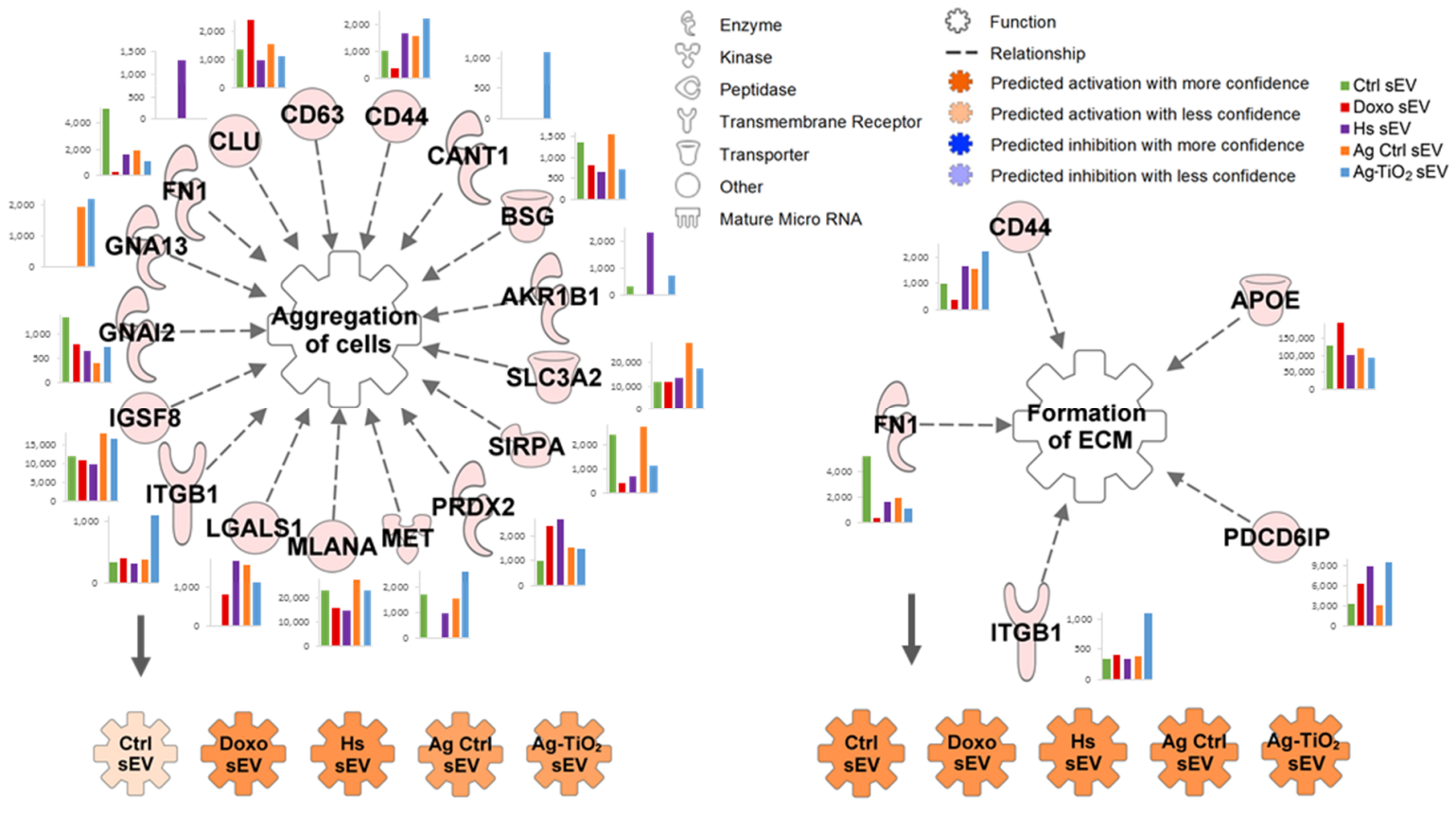

Figure 18. IPA predictions for the regulatory effects of sEV molecules on the 'Aggregation of cells' and the 'Formation of ECM'. Networks show every upstream regulator proteins accompanied by a bar graph, which represents the normalised expression values of the molecule for each $\mathrm{sEV}$ group. Coloured symbols named as the $\mathrm{sEV}$ groups display the expected regulation changes of the analysed 'Biofunctions' upon exposure to the vesicles.

These in silico predictions were also tested in vitro. In order to mimic in vivo conditions, we established a simplified 3D tumour matrix model co-culturing MSCs and B16F1 cells in hanging drop plates. By definition, multicellular cell aggregates, which produce their own ECM and are comprised of more than one cell types are termed as microtissues ${ }^{98}$. Applying an equal number of the two cell types (a total of 5,000 cells/well), the microtissue generation was followed under sEV exposures and DPBS treatment as a negative control for $72 \mathrm{~h}$. Images of the created microtissues were analysed by the AnaSP software developed for automatic image analysis of multicellular spheroids ${ }^{74}$. Each group of sEVs resulted in smaller and more compact microtissues, since the average values of the measured area, perimeter, diameter and volume was lower, compared to the negative Ctrl samples (Fig. 19). 
a

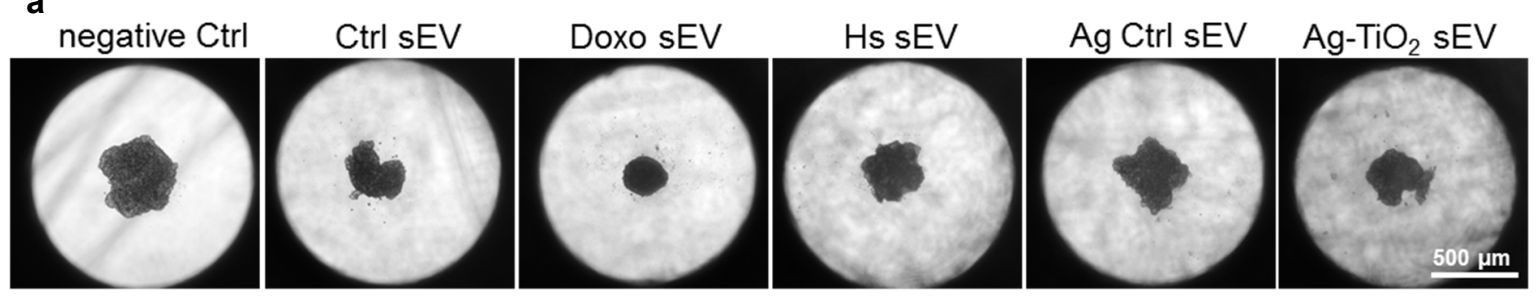

b

\begin{tabular}{|l|c|c|c|c|c|c|}
\hline Group name & negative Ctrl & Ctrl sEV & Doxo sEV & Hs sEV & Ag Ctrl sEV & Ag-TiO $_{2}$ sEV \\
\hline Eq. diameter $(\mu \mathrm{m})$ & $445 \pm 3$ & $315 \pm 7$ & $244 \pm 3$ & $351 \pm 11$ & $371 \pm 5$ & $338 \pm 7$ \\
\hline Major diameter $(\mu \mathrm{m})$ & $521 \pm 21$ & $370 \pm 7$ & $275 \pm 15$ & $430 \pm 31$ & $440 \pm 7$ & $393 \pm 3$ \\
\hline Minor diameter $(\mu \mathrm{m})$ & $307 \pm 56$ & $221 \pm 16$ & $204 \pm 14$ & $242 \pm 61$ & $287 \pm 20$ & $254 \pm 8$ \\
\hline Convexity & $0.801 \pm 0.033$ & $0.799 \pm 0.015$ & $0.911 \pm 0.024$ & $0.808 \pm 0.035$ & $0.779 \pm 0.013$ & $0.759 \pm 0.053$ \\
\hline Solidity & $0.885 \pm 0.022$ & $0.849 \pm 0.014$ & $0.942 \pm 0.019$ & $0.867 \pm 0.03$ & $0.859 \pm 0.019$ & $0.839 \pm 0.061$ \\
\hline Sphericity & $0.731 \pm 0.044$ & $0.701 \pm 0.011$ & $0.865 \pm 0.038$ & $0.727 \pm 0.052$ & $0.694 \pm 0.020$ & $0.667 \pm 0.061$ \\
\hline
\end{tabular}

c
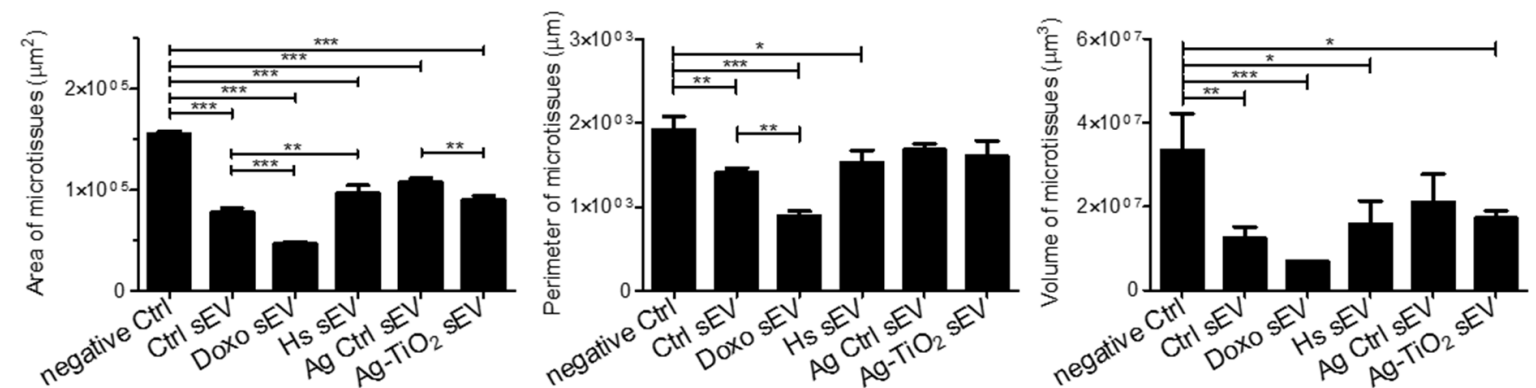

Figure 19. Microtissue generation of MSC-B16F1 co-cultures. (a) Representative images of the generated microtissues after $72 \mathrm{~h}$ of sEV exposures. (b) Descriptive statistics of the $72 \mathrm{~h} \mathrm{B16F1-MSC}$ microtissues resulted from image analysis using the AnaSP software. Eq. diameter means equivalent diameter, major and minor diameters are measured through centroid. Table contains mean $\pm \mathrm{SD}$ values. (c) Bar graphs show the area, perimeter and volume statistics of the generated microtissues (mean $+\mathrm{SD}, \mathrm{n}=3$ ), ${ }^{*} \mathrm{p}<0.05,{ }^{* *} \mathrm{p}<0.01$ and $* * * p<0.001$ indicate statistical significance.

These results were validated using mouse embryonic fibroblasts (MEFs) co-cultured with B16F1 cells, where each sEV group facilitated the microtissue generation, except the Hs sEVs (Suppl. Fig. S7). This means that surface to volume ratio was higher in the sEV-exposed microtissues, which may enhance the uptake of oxygen, growth factors and nutrients. At the same time, high compactness may reduce the penetration of drugs. 


\section{Discussion}

Several recent papers emphasize the mediating role of exosomes in tumorigenesis, tumour growth, metastasis, angiogenesis, and immune modulation in the tumour macro- and microenvironment ${ }^{13-15}$. However, effects of the therapy-induced stresses on the vesicular communication of tumour cells remain unclear.

In this study, we compared the sEV-mediated communication of NPC and melanoma cells under cytostatic and oxidative stress and cytostatic, heat and oxidative stress, respectively.

1. Using the NTA method, we demonstrated, that all type of stress conditions significantly increased the $\mathrm{sEV}$ production. However, this increase in the $\mathrm{sEV}$ release showed variety across the cell and stress types as well. For instance, the sEV production of melanoma cells was the highest under cytostatic stress, followed by the production under heat and oxidative stress.

Other papers also demonstrated that cells, including tumour cells release higher amount of exosomes in response to different types of stresses $^{99}$, such as hypoxia ${ }^{100}$, acidosis ${ }^{101}$, oxidative stress ${ }^{102}$, thermal stress ${ }^{102}$, radiation ${ }^{59,103}$ and cytotoxic drugs ${ }^{104,105}$. However, none of the previous studies have compared different types of stresses.

2. Sequencing of the small RNA content by the SOLiD technology showed substantial increase in the diversity of miRNA cargo of NPC-derived sEVs under both stress conditions. Sequencing and mass spectrometric analysis showed alterations in the molecular pattern of melanoma-derived sEVs upon exposure to different microenvironmental stresses. Several common molecules were also found in the sEVs, which were presented in the vesicles independently from the microenvironmental conditions.

These findings are in accordance with previous studies, which showed changes in the exosomal cargo upon exposure of the donor cells to external stimuli and stress conditions ${ }^{57,59,106}$. However, previous papers did not make a comparison of different stress conditions-induced sEV cargos. Furthermore, Peinado et al. ${ }^{44}$ and Lazar et al. ${ }^{107}$ analysing protein composition of different melanoma cell line-derived exosomes, also found melanocyte-specific proteins, transmembrane proteins, such as tetraspanins, transporters, and receptors as well as MVB and endosomal pathway-related proteins, for instance 
ESCRT-associated proteins, annexins, cytoskeletal and small GTP-binding proteins ${ }^{44,107}$. As these proteins were detected in each sEV group in this study as well, we were able to define a constant set of proteins of the melanoma-derived sEVs (Suppl. Fig. S1).

3. Using fluorescent spectroscopy, we presented that cytostatic stress-exposed melanoma cellderived sEVs might transfer doxorubicin to the recipient cells. However, the amount of transferred doxorubicin had not reached the $10 \%$ of median lethal dose (LD50=100 ng/ml $)^{75}$ for melanoma cells. At the same time, $\mathrm{Ag}-\mathrm{TiO}_{2}$ transfer was excluded by using DLS, CL $\mathrm{Ag}-\mathrm{TiO}_{2}$ detection method and TEM.

Theoretically, this low amount of transferred doxorubicin could not influence the function of Doxo sEVs in the recipient cells. However, a recent paper reported that doxorubicin encapsulated in exosomes had more potent cytotoxicity against the donor MCF-7 breast carcinoma cells than free drug ${ }^{108}$, suggesting that the transferred doxorubicin might influence the MSC proliferation and contribute to the tumour cell cycle arrest in our experiments.

$\mathrm{Ag}-\mathrm{TiO}_{2}$ nanoparticle transfer by sEVs has not been investigated in mammalian cells yet. However, Chen et al. showed, that macrophages accumulate $\mathrm{TiO}_{2}$ nanoparticles in $\mathrm{MVBs}^{109}$ (the endosomal compartments of exosome formation). At the same time, AnderssonWillman et al. could not detect $\mathrm{TiO}_{2}$ or $\mathrm{ZnO}$ particles in monocyte-derived dendritic cellreleased exosomes after nanoparticle exposures ${ }^{110}$. Schoelermann et al. observed that $\mathrm{TiO}_{2}$ transfer between normal rat kidney cells is a cellular contact-dependent phenomenon ${ }^{111}$.

4. We established a protocol for the bioinformatics analysis of $\mathrm{sEV}$ molecular patterns to predict their regulatory effects using the 'Grow' and 'MAP' tools of IPA. In this way, we developed an in silico model of sEV internalisation and activity in the recipient TME cells. In silico pathway analysis is often used in the EV research ${ }^{112-114}$, but here we developed a unique approach for the comprehensive and accurate prediction of the vesicular functions to compare the investigated sEV groups.

5. 'Core analysis' of IPA showed a huge overlap between the sEV groups in the 'Top 5 canonical pathways' and 'Top 5 molecular and cellular functions'. However, the subsequent 'Comparison analysis' revealed substantial differences between the sEV groups. Many 
intracellular, cellular, systemic and immune 'Biofunctions' were assigned to the sEV groups with different significance. Using our newly developed in silico model, we also found inverse regulatory effects between the sEVs released under different stress conditions.

There are only a few paper, which compare the effects of different microenvironmental conditions on the EV composition and function. Hedlund et al. demonstrated, that both thermal and oxidative stress - induced by $40^{\circ} \mathrm{C}$ and $\mathrm{H}_{2} \mathrm{O}_{2}-$ enhanced the release of immunosuppressive exosomes in human $\mathrm{T}$ cell leukemia Jurkat and $\mathrm{B}$ cell leukemia/lymphoma Raji cell lines ${ }^{102}$. De Jong et al. observed difference in the response of exosome-mediated communication of HMEC-1 endothelial cells to acute stress - such as hypoxia and tumour necrosis factor- $\alpha$ (TNF)- $\alpha$ - and short-term high sugar concentrations. Exposure of donor cells to hypoxia (model for ischemia) or TNF- $\alpha$ (model for inflammation and endothelial activation) modulated both protein and mRNA content of exosomes, whereas high glucose (model for hyperglycemia) or mannose (osmotic control for glucose) concentrations did not influence exosome molecular profiles ${ }^{115}$. Here, we performed a comprehensive analysis of the composition and function of sEVs based on the releasing cell types and microenvironmental conditions.

6. miRNA cargo of the NPC sEVs suggested, that NPC cells could not adapt to the microenvironmental stresses. In the in silico analyses, the sEV-transferred miRNAs under stress conditions were predicted for instance to inhibit the tumour cell migration instead of the original activation.

Melanoma-derived sEVs showed adaptive susceptibility to stresses as the sEV-delivered miRNAs and proteins were predicted, for instance to activate the tumour cell migration under cytostatic stress. These results suggest, that the microenvironmental stress response can be variable in the different tumour types. However, it is also possible that proteins play a greater role in the sEV-mediated communication, than miRNAs.

Adaptive properties of the sEV-mediated communication of tumour cells has not fully elucidated yet. However, there are some papers, which demonstrate tumour-promoting effects of stress-elicited EVs ${ }^{99}$. Ramteke et al. showed, that hypoxic condition-exposed prostate cancer cell lines (LNCaP and PC3) -released exosomes enhanced invasiveness, stemness and induced microenvironment changes; thereby, promoting tumour 
aggressiveness ${ }^{116}$. Angiogenesis-promoting of hypoxia-elicited exosomes was also demonstrated on hepatocellular carcinoma cells ${ }^{117}$. Keklikoglou et al. observed that two classes of cytotoxic drugs broadly employed in neoadjuvant breast cancer therapy (taxanes and anthracyclines) elicit tumour-derived EVs with enhanced pro-metastatic capacity. Preconditioning with chemotherapy-elicited 4T1 cell line-derived EVs, increased the number of metastatic lung nodules of $4 \mathrm{~T} 1$ cells in a lung colonisation assay performed in immunocompetent Balb/c mice ${ }^{53}$. Mutschelknaus et al. demonstrated that exosomes derived from irradiated HNSCC cells confer a migratory phenotype to recipient cancer cells. As underlying mechanism, they identified enhanced AKT-signalling by the radiation-regulated exosomal proteins ${ }^{57}$.

7. Trough in vitro experiments, we were able not only to predict the effects of melanomaderived sEV groups, but we also verified the functional differences between them. At this point, we investigated key functions of tumour progression, i.e. proliferation, cell cycle dynamics, migration and adhesion to describe the response patterns induced by the sEV groups.

Ctrl sEVs arrested the cell cycle and migration of tumour cells, but facilitated MSC proliferation, endothelial cell migration and microtissue generation of MSC/MEF-B16F1 co-cultures. In contrast, stress-elicited SEVs induced other effects on the recipient cells, but these effects were unique for each stress conditions. For instance, Doxo sEVs arrested melanoma cell cycle, but enhanced the tumour cell migration capacity, and showed the highest influence on microtissue generation, resulting the most compact structures. However, $\mathrm{Ag}-\mathrm{TiO}_{2}$ sEVs facilitated the melanoma cell migration, MSC proliferation and microtissue generation as well, without any kind of inhibitory effects. Our results suggest, that melanoma-derived sEVs induce diverse cellular functions in the recipient TME cells depending on the releasing conditions.

Previously, tumour exosomes derived from melanoma cells ${ }^{44}$, osteosarcoma cells ${ }^{118}$ or breast cancer cells ${ }^{119}$ have been shown to re-educate MSCs ensuring tumour-promoting properties for them. Tumour-derived EVs may target other cellular components of the TME, such as fibroblasts ${ }^{120-122}$ or endothelial cells ${ }^{123-125}$, thereby promoting tumour progression. 
There are also some papers reporting the alteration of the tumour-derived EV functions upon exposure to stress conditions, e.g. hypoxia, cytotoxic drugs or radiation, as we mentioned above. However, this study has no counterpart in the literature, since it compares different stress conditions and describes the $\mathrm{sEV}$-induced response patterns in different cell types of the TME. Our results highlight the functional complexity of tumour-derived sEVs, as we proved that releasing conditions can also increase the diversity of sEV effects.

8. The 'Biofunction' of 'Cell migration' was chosen to compare the response of different recipient cells to the melanoma-derived sEVs. In wound healing assays $\mathrm{Ctrl}$ and $\mathrm{Ag} \mathrm{Ctrl}$ sEVs showed inhibition on tumour cells, but activation on endothelial cell, as the in silico analyses predicted. Stress-exposed cell-derived exosomes reduced, abolished or inverted these effects, but these changes followed the IPA-predicted tendencies. In these experiments, we demonstrated for the first time, that cellular response to sEVs depends not only on the sEV cargo but on the recipient cell as well, which further increase the complexity and significance of the sEV-mediated communication in tumour progression. On the one hand, migration capacity of tumour cells has a great significance, since the process of tumour cell invasion and metastasis is conventionally understood as the migration of individual tumour cells that detach from the primary tumour, enter lymphatic vessels or the bloodstream and seed in distant organs ${ }^{126}$. On the other hand, endothelial cell migration as a key process of angiogenesis, is also an essential component of tumour $\operatorname{progression}^{127}$.

9. In this study, using our newly developed in silico model, we predicted sEV-induced cellular effects in the recipient cells, which were verified through in vitro assays, as we observed similar regulatory tendencies of the tumour-derived sEVs to the predictions. These results suggest that predictions resulted by the bioinformatics analyses may provide a good approximation of the therapy-induced host responses. 


\section{New findings}

1. Various types of stress conditions increase the sEV production of NPC and melanoma cells as well at different rates.

2. Number of miRNA types in the NPC sEVs increases by several fold under cytostatic and oxidative stress. Melanoma sEVs also deliver many condition-specific molecules, but miRNA and protein changes show unique patterns for each stress types. Here, we also defined the constant protein cargo of melanoma sEVs.

3. Melanoma cells, similarly to breast carcinoma cells, may transfer doxorubicin by sEVs, but cannot encapsulate $\mathrm{Ag}-\mathrm{TiO}_{2}$ nanoparticles.

4. Transfer and functional activity of the sEV cargo can be modelled in silico using the IPA approaches.

5. Different molecular patterns of tumour-derived sEVs, depending on the microenvironmental conditions, may show varying functional properties as well.

6. miRNA content of NPC sEVs promotes tumour progression, but it has inhibitory effects under stress conditions. At the same time, sEV-mediated communication of melanoma cells can adapt to the microenvironmental conditions.

7. Changes in the microenvironmental parameters is reflected in the sEVs-mediated communication of tumour cells through unique response patterns of the recipient cells.

8. The same melanoma sEV population, i.e. the same molecular cargo may induce opposite effects in different types of recipient cells.

9. Our newly developed in silico model may predict the sEV-induced effects with a good approximation, which can be useful not only in the research, but in a clinical setting as well.

\section{Conclusions}

In this study, we investigated effects of the microenvironmental changes on the sEV-mediated communication of tumour cells. We found that cytostatic, heat and oxidative stresses may alter the vesicular cargo, leading to different functional properties of the NPC- and melanomaderived SEVs in the recipient cells of the tumour matrix.

We showed, that sEVs, being complex information packages may participate in a wide range of signalling pathways. The fact, that a vesicular molecular pattern with a large number of molecules can influence the activation of the cellular homeostasis network at several points, 
suggests a huge diversity of sEV functions, which sometimes seem to be paradoxical in the in vitro experiments. In conclusion, hundreds of vesicular molecules may have thousands of functional effects in the recipient cells leading to an unconceivable outcome. Here, we successfully predicted the functional effects of the investigated sEV molecular patterns induced by five treatment conditions - by bioinformatics analyses using unique combinations of the IPA approaches. Based on our knowledge, our experimental setup was suitable to model the transfer and functional activity of the vesicular cargo in the recipient cells. Here, we demonstrated, that pathway analyses may provide a good approximation to the prediction of the therapy-induced stress responses, suggesting that in silico analyses may be useful tools not only in the field of research, but in a clinical setting as well.

This study provides data about the doxorubicin- and $\mathrm{Ag}$ - $\mathrm{TiO}_{2}$-elicited miRNA cargo of the NPC-derived sEVs and a detailed characterisation of the doxorubicin-, heat- and $\mathrm{Ag}-\mathrm{TiO}_{2}-$ induced molecular patterns of melanoma-derived sEVs as well. Furthermore, the melanoma sEV-induced response patterns were also characterised in the recipient cells. These results increase our knowledge about the molecular and functional complexity as well as conditiondependent variability of the NPC- and melanoma-derived sEVs. This study contributes to a better understanding of the pathogenesis and therapeutic responses of cancer diseases. It also highlights, that indirect effects of any therapy, such as a chemotherapy may have a great influence on the intercellular communication of the affected cells.

Based on this study, we concluded that the molecular pattern of these highly protected information packages is dictated by the microenvironmental conditions, including the therapeutic stress factors. The altered cargo of sEVs is able not only to enhance or suppress existing signalisation pathways, but even trigger de novo pathway activations, resulting a unique target cell-specific response pattern in the SEV recipient cells. Recent literature data $^{53,54,57,128}$ along with this study suggest that alteration of this complex sEV-mediated intercellular communication of tumour cells deserves special attention among the therapyinduced host responses, which may have a potential influence on the treatment efficacy. 


\section{References}

1. Doyle, L. M., \& Wang, M. Z. Overview of Extracellular Vesicles, Their Origin, Composition, Purpose, and Methods for Exosome Isolation and Analysis. Cells. 8, 727; https://doi.org/10.3390/cells8070727 (2019).

2. Zaborowski, M. P., Balaj, L., Breakefield, X. O., \& Lai, C. P. Extracellular Vesicles: Composition, Biological Relevance, and Methods of Study. Bioscience. 65, 783-797. DOI: https://doi.org/10.1093/biosci/biv084 (2015).

3. Yáñez-Mó, M. et al. Biological properties of extracellular vesicles and their physiological functions. J Extracell Vesicles. 4, 27066; https://doi.org/10.3402/jev.v4.27066 (2015).

4. Contreras-Naranjo, J. C., Wu, H. J., Ugaz, V. M. Microfluidics for exosome isolation and analysis: enabling liquid biopsy for personalized medicine. Lab Chip. 17, 3558-3577. DOI https://doi.org/10.1039/c7lc00592j (2017).

5. Hessvik, N. P. \& Llorente, A. Current knowledge on exosome biogenesis and release. Cell Mol Life Sci. 75, 193-208, DOI: https://doi.org/10.1007/s00018-017-2595-9 (2018).

6. Théry, C. et al. Minimal information for studies of extracellular vesicles 2018 (MISEV2018): a position statement of the International Society for Extracellular Vesicles and update of the MISEV2014 guidelines. $J$ Extracell Vesicles. 7, 1535750; https://doi.org/10.1080/20013078.2018.1535750 (2018).

7. Harding, C., Heuser, J. \& Stahl, P. Receptor-mediated endocytosis of transferrin and recycling of the transferrin receptor in rat reticulocytes. J. Cell Biol. 97, 329-339. DOI: https://doi.org/10.1083/jcb.97.2.329 (1983).

8. Pan, B. T. \& Johnstone, R. M. Fate of the transferrin receptor during maturation of sheep reticulocytes in vitro: selective externalization of the receptor. Cell. 33, 967-978. DOI: https://doi.org/10.1016/0092-8674(83)90040-5 (1983).

9. Whiteside, T. L. Tumor-Derived Exosomes and Their Role in Cancer Progression. Adv Clin Chem. 74, 103-141. DOI: https://doi.org/10.1016/bs.acc.2015.12.005 (2016).

10. Abels, E. R., Breakefield, X. O. Introduction to Extracellular Vesicles: Biogenesis, RNA Cargo Selection, Content, Release, and Uptake. Cell Mol Neurobiol. 36, 301-12. DOI: https://doi.org/10.1007/s10571-016-0366-z (2016). 
11. Maia, J., Caja, S., Strano Moraes, M. C., Couto, N. \& Costa-Silva, B. Exosome-Based CellCell Communication in the Tumor Microenvironment. Front Cell Dev Biol. 6, 18; https://doi.org/10.3389/fcell.2018.00018 (2018).

12. Kolenda, T. et al. Tumor microenvironment - Unknown niche with powerful therapeutic potential. Rep Pract Oncol Radiother. 23, 143-153, DOI: https://doi.org/10.1016/j.rpor.2018.01.004 (2018).

13. Meehan, K. \& Vella, L. J. The contribution of tumour-derived exosomes to the hallmarks of cancer. Crit Rev Clin Lab Sci. 53, 121-131, DOI: https://doi.org/10.3109/10408363.2015.1092496 (2016).

14. Javeed, N. \& Mukhopadhyay, D. Exosomes and their role in the micro-/macroenvironment: a comprehensive review. $J$ Biomed Res. 31, 386-394, DOI: https://doi.org/10.7555/JBR.30.20150162 (2017).

15. Tkach, M. \& Théry C. Communication by Extracellular Vesicles: Where We Are and Where We Need to Go. Cell. 164, 1226-1232, DOI: https://doi.org/10.1016/j.cell.2016.01.043 (2016).

16. Gulei, D. et al. Exosomes at a glance - common nominators for cancer hallmarks and novel diagnosis tools. Crit Rev Biochem Mol Biol. 53, 564-577. DOI: https://doi.org/10.1080/10409238.2018.1508276 (2018).

17. Li, K., Chen, Y., Li, A., Tan, C. \& Liu, X. Exosomes play roles in sequential processes of tumor metastasis. Int J Cancer. 144, 1486-1495. DOI: https://doi.org/10.1002/ijc.31774 (2019).

18. Sadovska, L., Santos, C. B., Kalnina, Z. \& Linē, A. Biodistribution, Uptake and Effects Caused by Cancer-Derived Extracellular Vesicles. J Circ Biomark. 4, 2; https://doi.org/10.5772/60522 (2015).

19. Kogure, T., Lin, W. L., Yan, I. K., Braconi, C., \& Patel, T. Intercellular nanovesiclemediated microRNA transfer: a mechanism of environmental modulation of hepatocellular cancer cell growth. Hepatology. 54, 1237-1248. DOI: https://doi.org/10.1002/hep.24504 (2011).

20. Corcoran, C. et al. Docetaxel-resistance in prostate cancer: evaluating associated phenotypic changes and potential for resistance transfer via exosomes. PloS One. 7, e50999; https://doi.org/10.1371/journal.pone.0050999 (2012). 
21. Taverna, S. et al. Role of exosomes released by chronic myelogenous leukemia cells in angiogenesis. Int $J$ Cancer. 130, 2033-2043. DOI: https://doi.org/10.1002/ijc.26217 (2012).

22. Hood, J. L., Pan, H., Lanza, G. M., Wickline, S. A., \& Consortium for Translational Research in Advanced Imaging and Nanomedicine (C-TRAIN). Paracrine induction of endothelium by tumor exosomes. Lab Invest. 89, 1317-1328. DOI: https://doi.org/10.1038/labinvest.2009.94 (2009).

23. Zhang, L. \& Yu, D. Exosomes in cancer development, metastasis, and immunity. Biochim Biophys Acta Rev Cancer. 1871, 455-468. DOI: https://doi.org/10.1016/j.bbcan.2019.04.004 (2019).

24. Lobb, R. J., Lima, L. G., Möller, A. Exosomes: Key mediators of metastasis and premetastatic niche formation. Semin Cell Dev Biol. 67, 3-10. DOI: https://doi.org/10.1016/j.semcdb.2017.01.004 (2017).

25. Ruivo, C. F., Adem, B., Silva, M. \& Melo, S. A. The Biology of Cancer Exosomes: Insights and New Perspectives. Cancer Res. 77, 6480-6488. DOI: https://doi.org/10.1158/00085472.CAN-17-0994 (2017).

26. Dos Anjos Pultz, B. et al. The multifaceted role of extracellular vesicles in metastasis: Priming the soil for seeding. Int $J$ Cancer. 140, 2397-2407. DOI: https://doi.org/10.1002/ijc.30595 (2017).

27. Brennan, B. Nasopharyngeal carcinoma. Orphanet $J$ Rare Dis. 1, 23. DOI: https://doi.org/10.1186/1750-1172-1-23 (2006).

28. Guo H, Huang S, Li S, Yu H, Wu S \& Zhou X. Prognostic significance of the long noncoding RNAs in nasopharyngeal carcinoma: a systematic review and meta-analysis. Cancer Manag Res. 27, 1763-1779. DOI: https://doi.org/10.2147/CMAR.S164695 (2018).

29. Zhou, Y. et al. Exosomes in Nasopharyngeal Carcinoma. J Cancer. 9, 767-777. DOI: https://doi.org/10.7150/jca.22505 (2018).

30. Klibi, J. et al. Blood diffusion and Th1-suppressive effects of galectin-9-containing exosomes released by Epstein-Barr virus-infected nasopharyngeal carcinoma cells. Blood. 113, 1957-1966. DOI: https://doi.org/10.1182/blood-2008-02-142596 (2009). 
31. Huang, S. C. M., Tsao, S. W. \& Tsang, C.M. Interplay of Viral Infection, Host Cell Factors and Tumor Microenvironment in the Pathogenesis of Nasopharyngeal Carcinoma. Cancers (Basel). 10, 106; https://doi.org/10.3390/cancers10040106 (2018).

32. Ye, S.B., et al. Tumor-derived exosomes promote tumor progression and T-cell dysfunction through the regulation of enriched exosomal microRNAs in human nasopharyngeal carcinoma. Oncotarget. 5, 5439-5452. DOI: https://doi.org/10.18632/oncotarget.2118 (2014).

33. Dukers, D. F. et al. Direct immunosuppressive effects of EBV-encoded latent membrane protein 1. J Immunol. 165, 663-70. DOI: https://doi.org/10.4049/jimmunol.165.2.663 (2000).

34. Aga, M. et al. Exosomal HIF1 $\alpha$ supports invasive potential of nasopharyngeal carcinomaassociated LMP1-positive exosomes. Oncogene. 33, 4613-4622. DOI: https://doi.org/10.1038/onc.2014.66 (2014).

35. You, Y. et al. Matrix metalloproteinase 13-containing exosomes promote nasopharyngeal carcinoma metastasis. Cancer Sci. 106, 1669-1677. DOI: https://doi.org/10.1111/cas.12818 (2015).

36. You, B. et al. Clinical and biological significance of hax-1 overexpression in nasopharyngeal carcinoma. Oncotarget. 7, 12505-12524. DOI: https://doi.org/10.18632/oncotarget.7274 (2016).

37. Schadendorf, D. et al. Melanoma. Lancet. 392, 971-984. DOI: https://doi.org/10.1016/S0140-6736(18)31559-9 (2018).

38. Braeuer, R. R. et al. Why is melanoma so metastatic? Pigment Cell Melanoma Res. 27, 1936, DOI: https://doi.org/10.1111/pcmr.12172 (2014).

39. Felicetti, F. et al. Exosome-mediated transfer of miR-222 is sufficient to increase tumor malignancy in melanoma. J Transl Med. 14, 56; https://doi.org/10.1186/s12967-016-0811$\underline{2}$ (2016).

40. Ekström, E.J. et al. WNT5A induces release of exosomes containing pro-angiogenic and immunosuppressive factors from malignant melanoma cells. Mol Cancer. 13, 88; https://doi.org/10.1186/1476-4598-13-88 (2014). 
41. Zhuang, G. et al. Tumour-secreted miR-9 promotes endothelial cell migration and angiogenesis by activating the JAK-STAT pathway. EMBO J. 31, 3513-3523. DOI: https://doi.org/10.1038/emboj.2012.183 (2012).

42. Hakulinen, J., Sankkila, L., Sugiyama, N., Lehti, K., Keski-Oja, J. Secretion of active membrane type 1 matrix metalloproteinase (MMP-14) into extracellular space in microvesicular exosomes. $J$ Cell Biochem. 105, 1211-1218. DOI: https://doi.org/10.1002/jcb.21923 (2008).

43. Tucci, M. et al. Exosomes in melanoma: a role in tumor progression, metastasis and impaired immune system activity. Oncotarget. 9, 20826-20837, DOI: https://doi.org/10.18632/oncotarget.24846 (2018).

44. Peinado, H. et al. Melanoma exosomes educate bone marrow progenitor cells toward a prometastatic phenotype through MET. Nat Med. 18, 883-891. DOI: https://doi.org/10.1038/nm.2753 (2012).

45. Peinado H. et al. Pre-metastatic niches: organ-specific homes for metastases. Nat Rev Cancer. 17, 302-317. DOI: https://doi.org/10.1038/nrc.2017.6 (2017).

46. Chen, G. et al. Exosomal PD-L1 contributes to immunosuppression and is associated with anti-PD-1 response. Nature. 560, 382-386. DOI: https://doi.org/10.1038/s41586-018$\underline{0392-8}$ (2018).

47. Shao, C. et al. Role of hypoxia-induced exosomes in tumor biology. Mol Cancer. 17, 120; https://doi.org/10.1186/s12943-018-0869-y (2018).

48. Meng, W, Hao, Y., He, C., Li, L. \& Zhu, G. Exosome-orchestrated hypoxic tumor microenvironment. Mol Cancer. 18，57; https://doi.org/10.1186/s12943-019-0982-6 (2019).

49. Parolini, I. et al. Microenvironmental $\mathrm{pH}$ is a key factor for exosome traffic in tumor cells. J Biol Chem. 284, 34211-22. DOI: https://doi.org/10.1074/jbc.M109.041152 (2009).

50. Mashouri, L. et al. Exosomes: composition, biogenesis, and mechanisms in cancer metastasis and drug resistance. Mol Cancer. 18, 75; https://doi.org/10.1186/s12943-0190991-5 (2019).

51. Shaked, Y. Balancing efficacy of and host immune responses to cancer therapy: the yin and yang effects. Nat Rev Clin Oncol. 13, 611-626, DOI: https://doi.org/10.1038/nrclinonc.2016.57 (2016). 
52. Milman, N., Ginini, L. \& Gil, Z. Exosomes and their role in tumorigenesis and anticancer drug resistance. Drug Resist Updat. 45, 1-12. DOI: https://doi.org/10.1016/j.drup.2019.07.003 (2019).

53. Keklikoglou, I. et al. Chemotherapy elicits pro-metastatic extracellular vesicles in breast cancer models. Nat Cell Biol. 21, 190-202, DOI: https://doi.org/10.1038/s41556-018-0256$\underline{3}(2019)$.

54. König, L. et al. Elevated levels of extracellular vesicles are associated with therapy failure and disease progression in breast cancer patients undergoing neoadjuvant chemotherapy. Oncoimmunology. 7, e1376153; https://doi.org/10.1080/2162402X.2017.1376153 (2017).

55. Lee, S. et al. Immunogenic Effect of Hyperthermia on Enhancing Radiotherapeutic Efficacy. Int J Mol Sci. 19, E2795; https://doi.org/10.3390/ijms19092795 (2018).

56. Mahmood, J. et al. Immunotherapy, Radiotherapy, and Hyperthermia: A Combined Therapeutic Approach in Pancreatic Cancer Treatment. Cancers (Basel). 10, E469; https://doi.org/10.3390/cancers10120469 (2018).

57. Mutschelknaus, L. et al. Radiation alters the cargo of exosomes released from squamous head and neck cancer cells to promote migration of recipient cells. Sci Rep. 7, 12423; https://doi.org/10.1038/s41598-017-12403-6 (2017).

58. Manda, G. et al. The redox biology network in cancer pathophysiology and therapeutics. Redox Biol. 5, 347-357, DOI: https://doi.org/10.1016/j.redox.2015.06.014 (2015).

59. Jelonek, K., Widlak, P. \& Pietrowska, M. The Influence of Ionizing Radiation on Exosome Composition, Secretion and Intercellular Communication. Protein Pept Lett. 23, 656-663, DOI: https://doi.org/10.2174/0929866523666160427105138 (2016).

60. Tallósy, S. P. et al. Investigation of the antibacterial effects of silver-modified $\mathrm{TiO}_{2}$ and $\mathrm{ZnO}$ plasmonic photocatalysts embedded in polymer thin films. Environ Sci Pollut Res Int. 21, 11155-11167, DOI: https://doi.org/10.1007/s11356-014-2568-6 (2014).

61. Veres, Á. et al. Silver and gold modified plasmonic $\mathrm{TiO}_{2}$ hybrid films for photocatalytic decomposition of ethanol under visible light. Catal Today. 181, 156-162, DOI: https://doi.org/10.1016/j.cattod.2011.05.028 (2012).

62. Hawley R. J., Kozlovac J. P. (2005) Decontamination. In: Lindler L. E., Lebeda F. J., Korch G. W. (eds) Biological Weapons Defense. Infectious Disease. Humana Press. https://doi.org/10.1385/1-59259-764-5:333. 
63. Tallósy, S. P. et al. Adhesion and inactivation of Gram-negative and Gram-positive bacteria on photoreactive $\mathrm{TiO}_{2} /$ polymer and $\mathrm{Ag}-\mathrm{TiO}_{2} /$ polymer nanohybrid films. Appl Surf Sci. 371, 139-150, DOI: https://doi.org/10.1016/j.apsusc.2016.02.202 (2016).

64. Szweda, P. et al. Essential Oils, Silver Nanoparticles and Propolis as Alternative Agents Against Fluconazole Resistant Candida albicans, Candida glabrata and Candida krusei Clinical Isolates. Indian J Microbiol. 55, 175-183, DOI: https://doi.org/10.1007/s12088014-0508-2 (2015).

65. Ahamed, M., Khan, M. A. M., Akhtar, M. J., Alhadlaq, H. A. \& Alshamsan, A. Ag-doping regulates the cytotoxicity of $\mathrm{TiO}_{2}$ nanoparticles via oxidative stress in human cancer cells. Sci Rep. 7, 17662; https://doi.org/10.1038/s41598-017-17559-9 (2017).

66. Tani, F. et al. Surface expression of a C-terminal alpha-helix region in heat shock protein 72 on murine LL/2 lung carcinoma can be recognized by innate immune sentinels. Mol Immunol. 46, 1326-1339, DOI: https://doi.org/10.1016/j.molimm.2008.11.020 (2009).

67. Hutter, J. L. \& Bechhoefer, J. Calibration of atomic-force microscope tips. Rev Sci Instrum. 64, 1868-1873. DOI: https://doi.org/10.1063/1.1143970 (1993).

68. Sader, J. E., Chon, J. W. M. \& Mulvaney, P. Calibration of rectangular atomic force microscope cantilevers. Rev Sci Instrum. 70, 3967-3969. DOI: https://doi.org/10.1063/1.1150021 (1999).

69. Higgins, M. J. et al. Noninvasive determination of optical lever sensitivity in atomic force microscopy. Rev Sci Instrum. 77, 013701; https://doi.org/10.1063/1.2162455 (2006).

70. Sader, J. E. et al. Spring constant calibration of atomic force microscope cantilevers of arbitrary shape. Rev Sci Instrum. 83, 103705; https://doi.org/10.1063/1.4757398 (2012).

71. Ebner, A., Hinterdorfer, P. \& Gruber, H. J. Comparison of different aminofunctionalization strategies for attachment of single antibodies to AFM cantilevers. Ultramicroscopy. 107, 922-927. DOI: https://doi.org/10.1016/j.ultramic.2007.02.035 (2007).

72. Aekbote, B. L. et al. Aminosilane-based functionalization of two-photon polymerized 3D SU-8 microstructures. Eur Polym J. 48, 1745-1754. DOI: https://doi.org/10.1016/j.eurpolymj.2012.06.011 (2012).

73. Toth, T. et al. Environmental properties of cells improve machine learning-based phenotype recognition accuracy. Sci Rep. 8, 10085; https://doi.org/10.1038/s41598-01828482-y (2018). 
74. Piccinini, F. AnaSP: a software suite for automatic image analysis of multicellular spheroids. Comput Methods Programs Biomed. 119, 43-52, DOI: https://doi.org/10.1016/j.cmpb.2015.02.006 (2015).

75. Zapata-Benavides, P. et al. WT1 silencing by RNAi synergizes with chemotherapeutic agents and induces chemosensitization to doxorubicin and cisplatin in B16F10 murine melanoma cells. Oncology letters. 3, 751-755, DOI: https://doi.org/10.3892/o1.2012.578 (2012).

76. Veres, Á. et al. Photocatalytic performance of silver-modified TiO2 embedded in poly(ethyl-acrylate-co-methyl metacrylate) matrix. Colloid Polym Sci. 292, 207-217, DOI: https://doi.org/10.1007/s00396-013-3063-1 (2014).

77. Tammali, R., Reddy, A. B., Srivastava, S. K. \& Ramana, K. V. Inhibition of aldose reductase prevents angiogenesis in vitro and in vivo. Angiogenesis. 14, 209-221, DOI: https://doi.org/10.1007/s10456-011-9206-4 (2011).

78. Sharma, M. et al. Developmental Competence of Buffalo (Bubalus bubalis) Pluripotent Embryonic Stem Cells Over Different Homologous Feeder Layers and the Comparative Evaluation with Various Extracellular Matrices. Int J Stem Cells. 6, 26-36, DOI: https://doi.org/10.15283/ijsc.2013.6.1.26 (2013).

79. Yamashita, A., Hakura, A. \& Inoue, H. Suppression of anchorage-independent growth of human cancer cell lines by the drs gene. Oncogene. 18, 4777-4787, DOI: https://doi.org/10.1038/sj.onc.1202852 (1999).

80. Tarasov, V. et al. Differential regulation of microRNAs by p53 revealed by massively parallel sequencing: miR-34a is a p53 target that induces apoptosis and G1-arrest. Cell Cycle. 6, 1586-1593, DOI: https://doi.org/10.4161/cc.6.13.4436 (2007).

81. Ji, Q. et al. MicroRNA miR-34 inhibits human pancreatic cancer tumor-initiating cells. PLoS One. 4, e6816; https://doi.org/10.1371/journal.pone.0006816 (2009).

82. Hibino, S. et al. Identification of an active site on the laminin alpha5 chain globular domain that binds to CD44 and inhibits malignancy. Cancer Res. 64, 4810-4816. DOI: https://doi.org/10.1158/0008-5472.CAN-04-0129 (2004).

83. Lee, D. J. et al. Peroxiredoxin-2 represses melanoma metastasis by increasing ECadherin/ $\beta$-Catenin complexes in adherens junctions. Cancer Res. 73, 4744-4757. doi: https://doi.org/10.1158/0008-5472.CAN-12-4226 (2013). 
84. Sekinem Y. et al. STAP-2 Protein Expression in B16F10 Melanoma Cells Positively Regulates Protein Levels of Tyrosinase, Which Determines Organs to Infiltrate in the Body. J Biol Chem. 290, 17462-17473. DOI: https://doi.org/10.1074/jbc.M115.658575 (2015).

85. Fu, T. Y. et al. Let-7b-mediated suppression of basigin expression and metastasis in mouse melanoma cells. Exp Cell Res. 317, 445-451. DOI: https://doi.org/10.1016/j.yexcr.2010.11.004 (2011).

86. Kappelmann, M., Kuphal, S., Meister, G., Vardimon, L. \& Bosserhoff, A. K. MicroRNA miR-125b controls melanoma progression by direct regulation of c-Jun protein expression. Oncogene. 32, 2984-2991. DOI: https://doi.org/10.1038/onc.2012.307 (2013).

87. Yan, D. et al. MicroRNA-34a inhibits uveal melanoma cell proliferation and migration through downregulation of c-Met. Invest Ophthalmol Vis Sci. 50, 1559-1565. DOI: https://doi.org/10.1167/iovs.08-2681 (2009).

88. Zhang, P. et al. CD82 suppresses CD44 alternative splicing-dependent melanoma metastasis by mediating U2AF2 ubiquitination and degradation. Oncogene. 35, 5056-5069. DOI: https://doi.org/10.1038/onc.2016.67 (2016).

89. Marzese, D. M. et al. Brain metastasis is predetermined in early stages of cutaneous melanoma by CD44v6 expression through epigenetic regulation of the spliceosome. Pigment Cell Melanoma Res. 28, 82-93. DOI: https://doi.org/10.1111/pcmr.12307 (2015).

90. Hernández, D. et al. V3 versican isoform alters the behavior of human melanoma cells by interfering with CD44/ErbB-dependent signaling. $J$ Biol Chem. 286, 1475-85. DOI: https://doi.org/10.1074/jbc.M110.127522 (2011).

91. Thomas, L., Byers, H. R., Vink, J. \& Stamenkovic, I. CD44H regulates tumor cell migration on hyaluronate-coated substrate. $J$ Cell Biol. 118, 971-977. DOI: https://doi.org/10.1083/jcb.118.4.971 (1992).

92. Hong, I. K. et al. The tetraspanin CD81 protein increases melanoma cell motility by upregulating metalloproteinase MT1-MMP expression through the pro-oncogenic Aktdependent Sp1 activation signaling pathways. J Biol Chem. 289, 15691-15704. DOI: https://doi.org/10.1074/jbc.M113.534206 (2014). 
93. Hibino, T. et al. S100A9 is a novel ligand of EMMPRIN that promotes melanoma metastasis. Cancer Res. 73, 172-183. doi: https://doi.org/10.1158/0008-5472.CAN-11$\underline{3843}$ (2013).

94. Hodgson, L., Henderson, A. J. \& Dong, C. Melanoma cell migration to type IV collagen requires activation of NF-kappaB. Oncogene. 22, 98-108. DOI: https://doi.org/10.1038/sj.onc.1206059 (2003).

95. Maaser, K. et al. Functional hierarchy of simultaneously expressed adhesion receptors: integrin alpha2beta1 but not CD44 mediates MV3 melanoma cell migration and matrix reorganization within three-dimensional hyaluronan-containing collagen matrices. $\mathrm{Mol}$ Biol Cell. 10, 3067-79. DOI: https://doi.org/10.1091/mbc.10.10.3067 (1999).

96. Stampolidis, P., Ullrich, A. \& Iacobelli, S. LGALS3BP, lectin galactoside-binding soluble 3 binding protein, promotes oncogenic cellular events impeded by antibody intervention. Oncogene. 34, 39-52, DOI: https://doi.org/10.1038/onc.2013.548 (2015).

97. Pan, S. et al. Extracellular Alix regulates integrin-mediated cell adhesions and extracellular matrix assembly. EMBO J. 27, 2077-2090, DOI: https://doi.org/10.1038/emboj.2008.134 (2008).

98. Piccinini, F., Santis, I. \& Bevilacqua, A. Advances in cancer modeling: fluidic systems for increasing representativeness of large 3D multicellular spheroids. Biotechniques. 65, 312 314. DOI: https://doi.org/10.2144/btn-2018-0153 (2018).

99. Kucharzewska, P. \& Belting, M. Emerging roles of extracellular vesicles in the adaptive response of tumour cells to microenvironmental stress. J Extracell Vesicles. 2, 10.3402/jev.v2i0.20304; https://doi.org/10.3402/jev.v2i0.20304 (2013).

100. King, H. W., Michael, M.Z. \& Gleadle, J.M. Hypoxic enhancement of exosome release by breast cancer cells. BMC Cancer. 12, 421; https://doi.org/10.1186/1471-2407-12-421 (2012).

101. Parolini, I. et al. Microenvironmental $\mathrm{pH}$ is a key factor for exosome traffic in tumor cells. J Biol Chem. 284, 34211-34222, DOI: https://doi.org/10.1074/jbc.M109.041152 (2009).

102. Hedlund, M., Nagaeva, O., Kargl D., Baranov. V. \& Mincheva-Nilsson L. Thermal- and oxidative stress causes enhanced release of NKG2D ligand-bearing immunosuppressive exosomes in leukemia/lymphoma $\mathrm{T}$ and $\mathrm{B}$ cells. PLoS One. 6, e16899; https://doi.org/10.1371/journal.pone.0016899 (2011). 
103. Jelonek, K. et al. Ionizing radiation affects protein composition of exosomes secreted in vitro from head and neck squamous cell carcinoma. Acta Biochim Pol. 62, 265-272. DOI: http://dx.doi.org/10.18388/abp.2015 970 (2015).

104. Lv, L. H. et al. Anticancer drugs cause release of exosomes with heat shock proteins from human hepatocellular carcinoma cells that elicit effective natural killer cell antitumor responses in vitro. $J$ Biol Chem. 287, 15874-15885, DOI: https://doi.org/10.1074/jbc.M112.340588 (2012).

105. Yang, Y., Chen, Y., Zhang, F., Zhao, Q. \& Zhong, H. Increased anti-tumour activity by exosomes derived from doxorubicin-treated tumour cells via heat stress. Int $J$ Hyperthermia. 31, 498-506, DOI: https://doi.org/10.3109/02656736.2015.1036384 (2015).

106. Wozniak, M., Peczek, L., Czernek, L. \& Düchler, M. Analysis of the miRNA Profiles of Melanoma Exosomes Derived Under Normoxic and Hypoxic Culture Conditions. Anticancer Res. 37, 6779-6789, DOI: https://doi.org/10.21873/anticanres.12138 (2017).

107. Lazar, I. et al. Proteome characterization of melanoma exosomes reveals a specific signature for metastatic cell lines. Pigment Cell Melanoma Res. 28, 464-475, DOI: https://doi.org/10.1111/pcmr.12380 (2015).

108. Yang, Y., Chen, Y., Zhang, F., Zhao, Q. \& Zhong, H. Increased anti-tumour activity by exosomes derived from doxorubicin-treated tumour cells via heat stress. Int $J$ Hyperthermia. 31, 498-506, DOI: https://doi.org/10.3109/02656736.2015.1036384 (2015).

109. Chen, Q. et al. $\mathrm{TiO}_{2}$ nanoparticles cause mitochondrial dysfunction, activate inflammatory responses, and attenuate phagocytosis in macrophages: A proteomic and metabolomic insight. Redox Biol. 15, 266-276. DOI: https://doi.org/10.1016/j.redox.2017.12.011 (2018).

110. Andersson-Willman, B. et al. Effects of subtoxic concentrations of $\mathrm{TiO} 2$ and $\mathrm{ZnO}$ nanoparticles on human lymphocytes, dendritic cells and exosome production. Toxicol Appl Pharmacol. 264, 94-103. DOI: https://doi.org/10.1016/j.taap.2012.07.021 (2012).

111. Schoelermann, J., Burtey, A., Allouni, Z. E., Gerdes, H. H. \& Cimpan, M. R. Contactdependent transfer of $\mathrm{TiO}_{2}$ nanoparticles between mammalian cells. Nanotoxicology. 10, 204-215. DOI: https://doi.org/10.3109/17435390.2015.1048322 (2016). 
112. Dozio, V. \& Sanchez, J. C. Characterisation of extracellular vesicle-subsets derived from brain endothelial cells and analysis of their protein cargo modulation after TNF exposure. J Extracell Vesicles. 6, 1302705; https://doi.org/10.1080/20013078.2017.1302705 (2017).

113. Vykoukal, J. et al. Plasma-derived extracellular vesicle proteins as a source of biomarkers for lung adenocarcinoma. Oncotarget. 8, 95466-95480; https://doi.org/10.18632/oncotarget.20748 (2017).

114. Chen, M. et al. Transcriptome and long noncoding RNA sequencing of three extracellular vesicle subtypes released from the human colon cancer LIM1863 cell line. Sci Rep. 6, 38397; https://doi.org/10.1038/srep38397 (2016).

115. de Jong, O. G. et al. Cellular stress conditions are reflected in the protein and RNA content of endothelial cell-derived exosomes. J Extracell Vesicles. 1, 10.3402/jev.v1i0.18396; https://doi.org/10.3402/jev.v1i0.18396 (2012).

116. Ramteke, A. et al. Exosomes secreted under hypoxia enhance invasiveness and stemness of prostate cancer cells by targeting adherens junction molecules. Mol Carcinog. 54, 554-565. DOI: https://doi.org/10.1002/mc.22124 (2015).

117. Matsuura, Y. et al. Exosomal miR-155 Derived from Hepatocellular Carcinoma Cells Under Hypoxia Promotes Angiogenesis in Endothelial Cells. Dig Dis Sci. 64, 792-802. DOI: https://doi.org/10.1007/s10620-018-5380-1 (2019).

118. Baglio, S. R. et al. Blocking Tumor-Educated MSC Paracrine Activity Halts Osteosarcoma Progression. Clin Cancer Res. 23, 3721-3733, DOI: https://doi.org/10.1158/1078-0432.CCR-16-2726 (2017).

119. Cho, J. A., Park, H., Lim, E. H. \& Lee, K. W. Exosomes from breast cancer cells can convert adipose tissue-derived mesenchymal stem cells into myofibroblast-like cells. Int $J$ Oncol. 40, 130-138, DOI: https://doi.org/10.3892/ijo.2011.1193 (2012).

120. Shu, S. L. et al. Metabolic reprogramming of stromal fibroblasts by melanoma exosome microRNA favours a pre-metastatic microenvironment. Sci Rep. 8, 12905; https://doi.org/10.1038/s41598-018-31323-7 (2018).

121.Zhou, X. et al. Melanoma cell-secreted exosomal miR-155-5p induce proangiogenic switch of cancer-associated fibroblasts via SOCS1/JAK2/STAT3 signaling pathway. $J$ Exp Clin Cancer Res. 37, 242; https://doi.org/10.1186/s13046-018-0911-3 (2018). 
122. Yoshii, S. et al. Exosomal microRNAs derived from colon cancer cells promote tumor progression by suppressing fibroblast TP53 expression. Cancer Sci. 110, 2396-2407. DOI: https://doi.org/10.1111/cas.14084 (2019).

123. Huaitong, X. et al. Microvesicles releasing by oral cancer cells enhance endothelial cell angiogenesis via Shh/RhoA signaling pathway. Cancer Biol Ther. 18, 783-791. DOI: https://doi.org/10.1080/15384047.2017.1373213 (2017).

124. Lawson, J. et al. Selective secretion of microRNAs from lung cancer cells via extracellular vesicles promotes CAMK1D-mediated tube formation in endothelial cells. Oncotarget. 8, 83913-83924. DOI: https://doi.org/10.18632/oncotarget.19996 (2017).

125. Bao, L. et al. Metastasis-associated miR-23a from nasopharyngeal carcinoma-derived exosomes mediates angiogenesis by repressing a novel target gene TSGA10. Oncogene. 37, 2873-2889. DOI: https://doi.org/10.1038/s41388-018-0183-6 (2018).

126. Friedl, P. \& Wolf, K. Tumour-cell invasion and migration: diversity and escape mechanisms. Nat Rev Cancer. 3, 362-374, DOI: https://doi.org/10.1038/nrc1075 (2003).

127. Lamalice, L., Le Boeuf, F. \& Huot, J. Endothelial cell migration during angiogenesis. Circ Res. 100, 782-794, DOI: https://doi.org/10.1161/01.RES.0000259593.07661.1e (2007).

128. Osti, D. et al. Clinical Significance of Extracellular Vesicles in Plasma from Glioblastoma Patients. Clin Cancer Res. 25, 266-276, DOI: https://doi.org/10.1158/1078-0432.CCR-181941 (2019). 


\section{Acknowledgements}

First of all, I would like to express my most sincere gratitude and appreciation to my supervisor, Krisztina Buzás, PhD for her guidance, patience and encouragement throughout my PhD studies. I also wish to thank her for introducing me to the world of scientific research and for teaching me to think like a real researcher.

I am grateful to the head of the Institute of Biochemistry and the Laboratory of Image Analysis and Machine Learning in the BRC, Péter Horváth, PhD for the scientific and infrastructural support of my project.

I wish to thank my colleagues, Gabriella Dobra, Edina Gyukity-Sebestyén, Lilla Pintér, Mária Kovács for the scientific, technical and personal support. Writing this dissertation would not have been possible without their continuous help. I also thank any support for the other members of the Laboratory of Image Analysis and Machine Learning.

I thank all colleagues in the Biological Research Centre, who supported me with constructive ideas or provided laboratory materials or equipment.

I wish to thank all of the co-authors of my publications for their contributions to the scientific research. I am grateful to Filippo Piccinini, PhD for introducing me to the world of 3D cell cultures.

I also thank the financial support as my research was supported by the GINOP-2.3.2-15-201600015; GINOP-2.2.1-15-2017-00052, NKFI-6-K-11493 and the University of Szeged Open Access Fund.

Finally, I would like to thank my dear, Zoltán Árpád Váradi for not only the IT support, but the continuous personal support. I am grateful to my family and relatives, who missed me a lot during the last years. 


\section{Supplementary Figures}

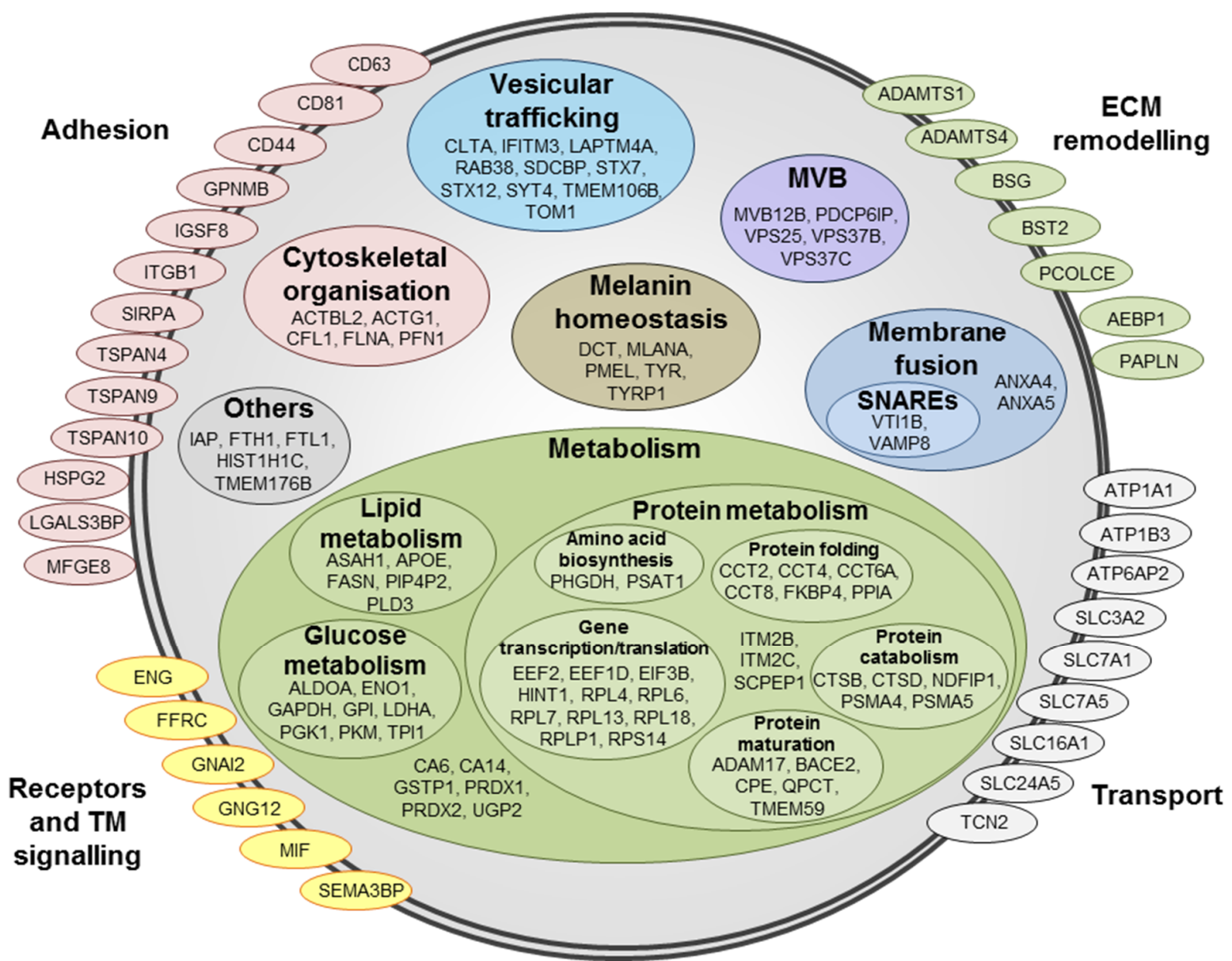

Supplementary Figure S1. Classification of the common proteins for each melanoma sEV group based on their function and localisation in vesicles. 


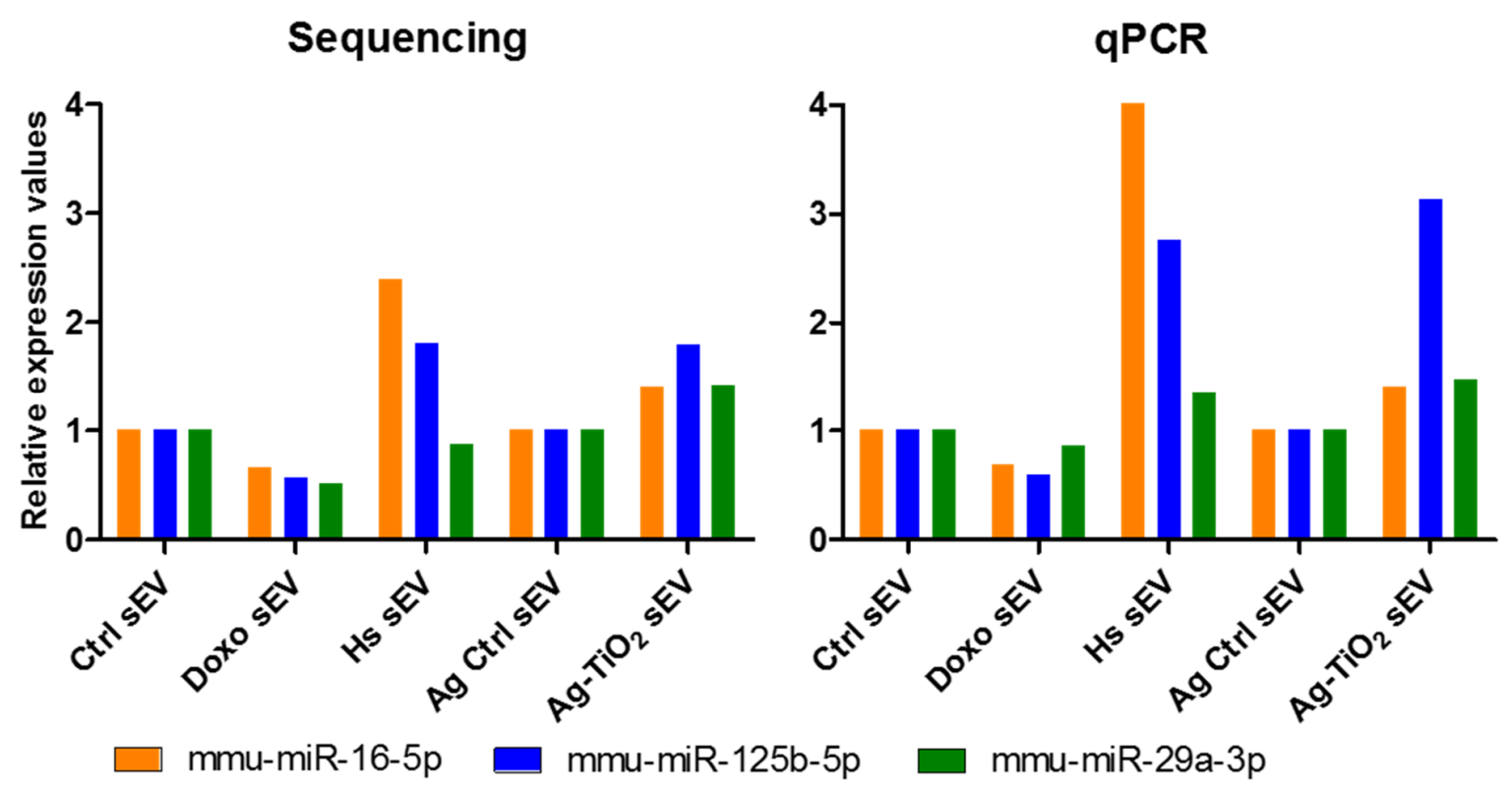

Supplementary Figure S2. Validation of the miRNA sequencing results by qPCR. Relative expression values of mmu-miR-16-5p, mmu-miR-125b-5p and mmu-miR-29a-3p showed similar patterns for both quantification methods. 

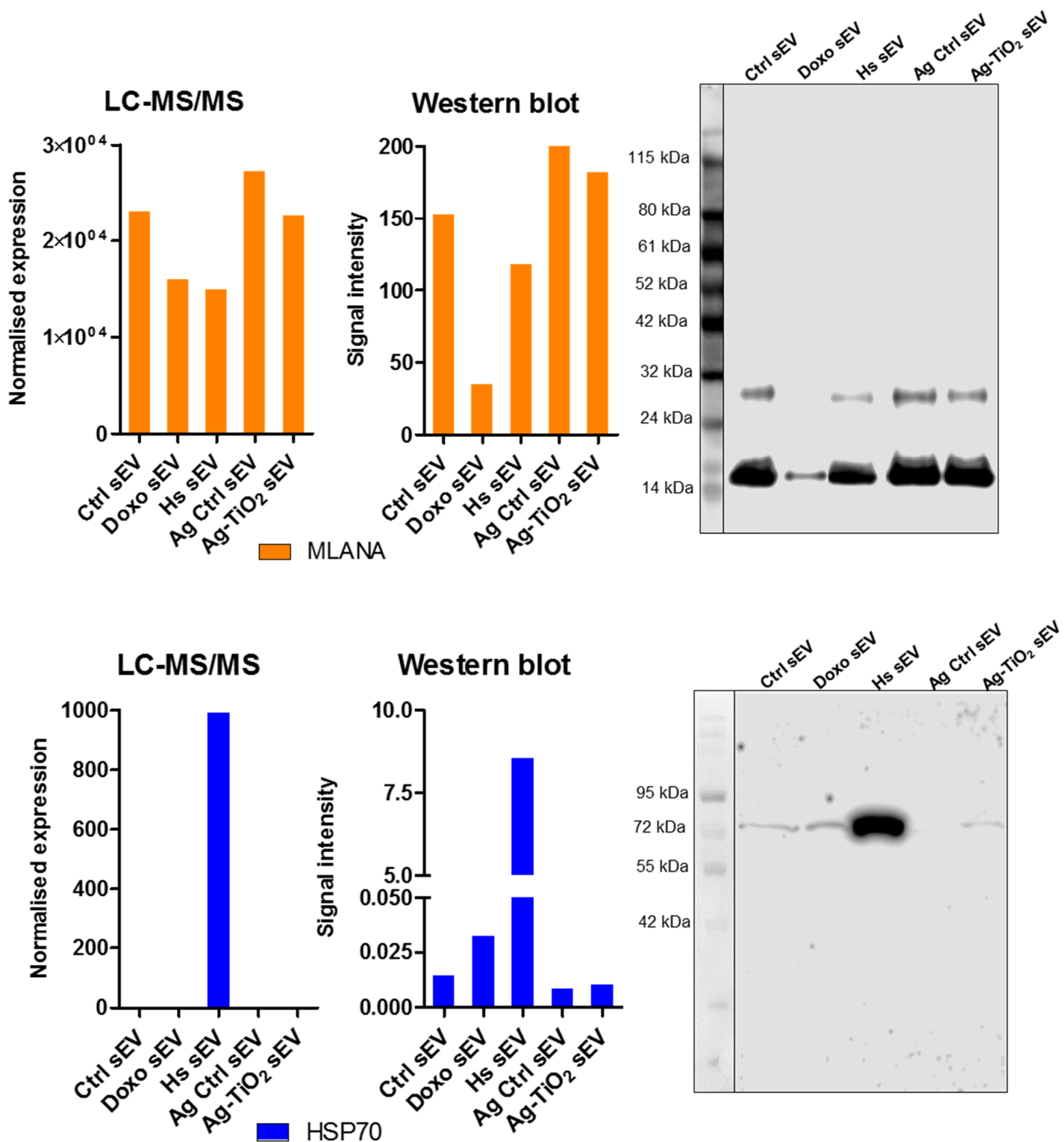

Supplementary Figure S3. Validation of the LC-MS/MS results by Western blot analyses. Signal intensities of HSP70 and MLANA were quantified by densitometry, which reflected the LC-MS/MS resulted differences between the sEV groups. However, using Western blot, which is a more sensitive method, we were able to detect HSP70 in each sEV isolates. sEV and Cell samples were investigated on the same gels/blots for each marker, however their lines were cropped, shown by black boxes. 


\begin{tabular}{|l|c|c|c|c|c|}
\hline \multirow{2}{*}{\multicolumn{1}{|c|}{ Top 5 canonical pathways }} & \multicolumn{5}{c|}{ p-value } \\
\cline { 2 - 6 } & Ctrl sEV & Doxo sEV & Hs sEV & $\begin{array}{c}\text { Ag Ctrl } \\
\text { sEV }\end{array}$ & $\begin{array}{c}\text { Ag-TiO } \\
\text { sEV }\end{array}$ \\
\hline Glycolysis I & $3.22 \mathrm{E}-08$ & $9.50 \mathrm{E}-09$ & $1.37 \mathrm{E}-08$ & $2.33 \mathrm{E}-08$ & $5.50 \mathrm{E}-08$ \\
\hline Gluconeogenesis I & $1.93 \mathrm{E}-05$ & $8.21 \mathrm{E}-06$ & $1.06 \mathrm{E}-05$ & $1.54 \mathrm{E}-05$ & $2.81 \mathrm{E}-05$ \\
\hline Eumelanin biosynthesis & $2.17 \mathrm{E}-05$ & $1.27 \mathrm{E}-05$ & $1.50 \mathrm{E}-05$ & $1.88 \mathrm{E}-05$ & $2.74 \mathrm{E}-05$ \\
\hline Phagosome maturation & $1.48 \mathrm{E}-04$ & $3.80 \mathrm{E}-05$ & $5.75 \mathrm{E}-05$ & $5.52 \mathrm{E}-04$ & $5.02 \mathrm{E}-05$ \\
\hline $\begin{array}{l}\text { Inhibition of matrix } \\
\text { metalloproteinases }\end{array}$ & $1.64 \mathrm{E}-03$ & $6.79 \mathrm{E}-02$ & $1.04 \mathrm{E}-03$ & $1.38 \mathrm{E}-03$ & $2.18 \mathrm{E}-03$ \\
\hline EIF2 signalling & $1.02 \mathrm{E}-02$ & $4.36 \mathrm{E}-05$ & $4.32 \mathrm{E}-07$ & $1.09 \mathrm{E}-06$ & $4.68 \mathrm{E}-06$ \\
\hline
\end{tabular}

b

\begin{tabular}{|l|c|c|c|c|c|}
\hline \multirow{2}{*}{$\begin{array}{c}\text { Top } 5 \text { molecular and cellular } \\
\text { functions }\end{array}$} & \multicolumn{5}{|c|}{ p-range } \\
\cline { 2 - 6 } & Ctrl sEV & Doxo sEV & Hs sEV & $\begin{array}{c}\text { Ag Ctrl } \\
\text { sEV }\end{array}$ & $\begin{array}{c}\text { Ag-TiO } \\
\text { sEV }\end{array}$ \\
\hline \multirow{2}{*}{ Cellular development } & $4.45 \mathrm{E}-04-$ & $3.08 \mathrm{E}-04-$ & $1.67 \mathrm{E}-04-$ & $4.09 \mathrm{E}-04-$ & $5.60 \mathrm{E}-04-$ \\
& $5.35 \mathrm{E}-20$ & $3.60 \mathrm{E}-19$ & $2.51 \mathrm{E}-19$ & $8.18 \mathrm{E}-20$ & $3.52 \mathrm{E}-20$ \\
\hline \multirow{2}{*}{ Cellular growth and proliferation } & $4.45 \mathrm{E}-04-$ & $3.08 \mathrm{E}-04-$ & $1.67 \mathrm{E}-04-$ & $4.09 \mathrm{E}-04-$ & $5.60 \mathrm{E}-04-$ \\
& $5.35 \mathrm{E}-20$ & $3.60 \mathrm{E}-19$ & $2.51 \mathrm{E}-19$ & $8.18 \mathrm{E}-20$ & $3.52 \mathrm{E}-20$ \\
\hline \multirow{2}{*}{ Cell death and survival } & $4.82 \mathrm{E}-04-$ & $2.47 \mathrm{E}-04-$ & $1.73 \mathrm{E}-04-$ & $3.89 \mathrm{E}-04-$ & $5.92 \mathrm{E}-04-$ \\
& $7.73 \mathrm{E}-20$ & $2.40 \mathrm{E}-21$ & $7.89 \mathrm{E}-25$ & $3.15 \mathrm{E}-19$ & $1.53 \mathrm{E}-18$ \\
\hline \multirow{2}{*}{ Cellular movement } & $4.45 \mathrm{E}-04-$ & $3.08 \mathrm{E}-04-$ & $1.81 \mathrm{E}-04-$ & $4.31 \mathrm{E}-04-$ & $5.88 \mathrm{E}-04-$ \\
& $3.58 \mathrm{E}-15$ & $5.11 \mathrm{E}-14$ & $5.03 \mathrm{E}-12$ & $5.97 \mathrm{E}-14$ & $3.58 \mathrm{E}-14-$ \\
\hline Cell-to-cell signalling and & $4.81 \mathrm{E}-04-$ & $3.05 \mathrm{E}-04-$ & $1.77 \mathrm{E}-04-$ & $4.02 \mathrm{E}-04-$ & $5.92 \mathrm{E}-04-$ \\
interaction & $2.71 \mathrm{E}-07$ & $1.12 \mathrm{E}-07$ & $2.40 \mathrm{E}-08$ & $3.77 \mathrm{E}-08$ & $3.99 \mathrm{E}-07$ \\
\hline \multirow{2}{*}{ Protein synthesis } & $1.53 \mathrm{E}-04-$ & $3.12 \mathrm{E}-04-$ & $1.96 \mathrm{E}-04-$ & $9.31 \mathrm{E}-05-$ & $9.89 \mathrm{E}-05-$ \\
& $4.69 \mathrm{E}-06$ & $4.21 \mathrm{E}-08$ & $1.55 \mathrm{E}-12$ & $5.51 \mathrm{E}-07$ & $1.00 \mathrm{E}-06$ \\
\hline
\end{tabular}

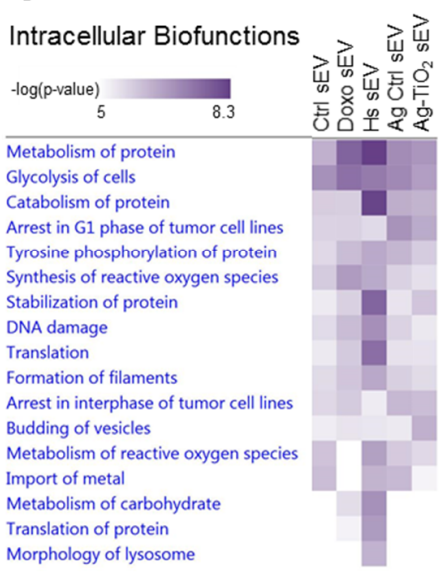

d

Cellular Biofunctions

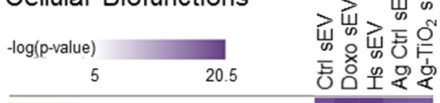

Necrosis

Cell proliferation of tumor cell lines Cell death

Apoptosis of tumor cell lines

Cell movement of tumor cell lines

Migration of tumor cell lines

Apoptosis

Cell death of cancer cells

Cell death of tumor cells

Cell movement

Cell viability of tumor cell lines

Cell movement of melanoma cell lines

Cell viability

Cell survival

Migration of cells

Migration of melanoma cell lines

Interaction of tumor cell lines

Apoptosis of tumor cells

Binding of tumor cell lines

Cell movement of endothelial cells

Migration of endothelial cells

Apoptosis of cancer cells

Detachment of cells

Size of cells

Cell spreading of endothelial cells

Colony formation of tumor cell lines

Formation of extracellular matrix

Quantity of cells

Proliferation of tumor cells

Endothelial cell development

Movement of vascular endothelial cells

Cell proliferation of fibroblasts

Binding of endothelial cells

Cell death of connective tissue cells

Cellular homeostasis

Supplementary Figure S4. IPA showed that sEVs can influence many biological pathways and functions with different significance via their miRNA and protein content. (a) 'Top 5 canonical pathways' for each sEV group. Red values label the significance of pathways, which were not included in the Top 5. (b) 'Top 5 molecular and cellular functions' for each sEV group. Red values label the significance ranges of functions, which were not included in the Top 5. (c-f) Heat maps from the 'Comparison analysis' of the molecular content of vesicles. Relevant 'Biofunctions' with $\mathrm{p} \leq 0.00001$ were organised into four groups, namely intracellular, cellular, systemic and immune processes. e

Systemic Biofunctions
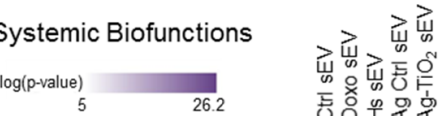

否各尔是是

Early stage tumor

Early stage solid tumor

Advanced malignant tumor

Primary melanom

Advanced malignant solid tumor

Metastatic solid tumor

Invasion of cells

Primary solid tumor

Stage I-II cancer

Metastatic melanom

Growth of tumor

Fibrosis

Metastasis of cells

Angiogenesis

Stage I cancer

Development of vasculature

Cancer of cells

Angiogenesis of tumor

Fibrogenesis

Progression of tumor

Malignant solid tur

Growth of malignant tumo

Solid tumor f

Immune Biofunctions

등음오웡

Transmigration of leukocytes

Activation of blood cells

Cell movement of monocytes

Transmigration of cells

Detachment of blood cells

Cell rolling of leukocytes

Cell movement of phagocytes

Detachment of granulocytes

Immune response of tu

Binding of leukocytes

Adhesion of blood cells

Migration of granulocytes

Adhesion of immune cells

Cell tethering or rolling of leukocytes

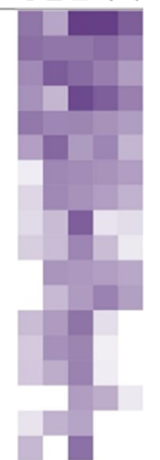

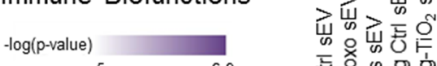

Activation of leukocytes 
a

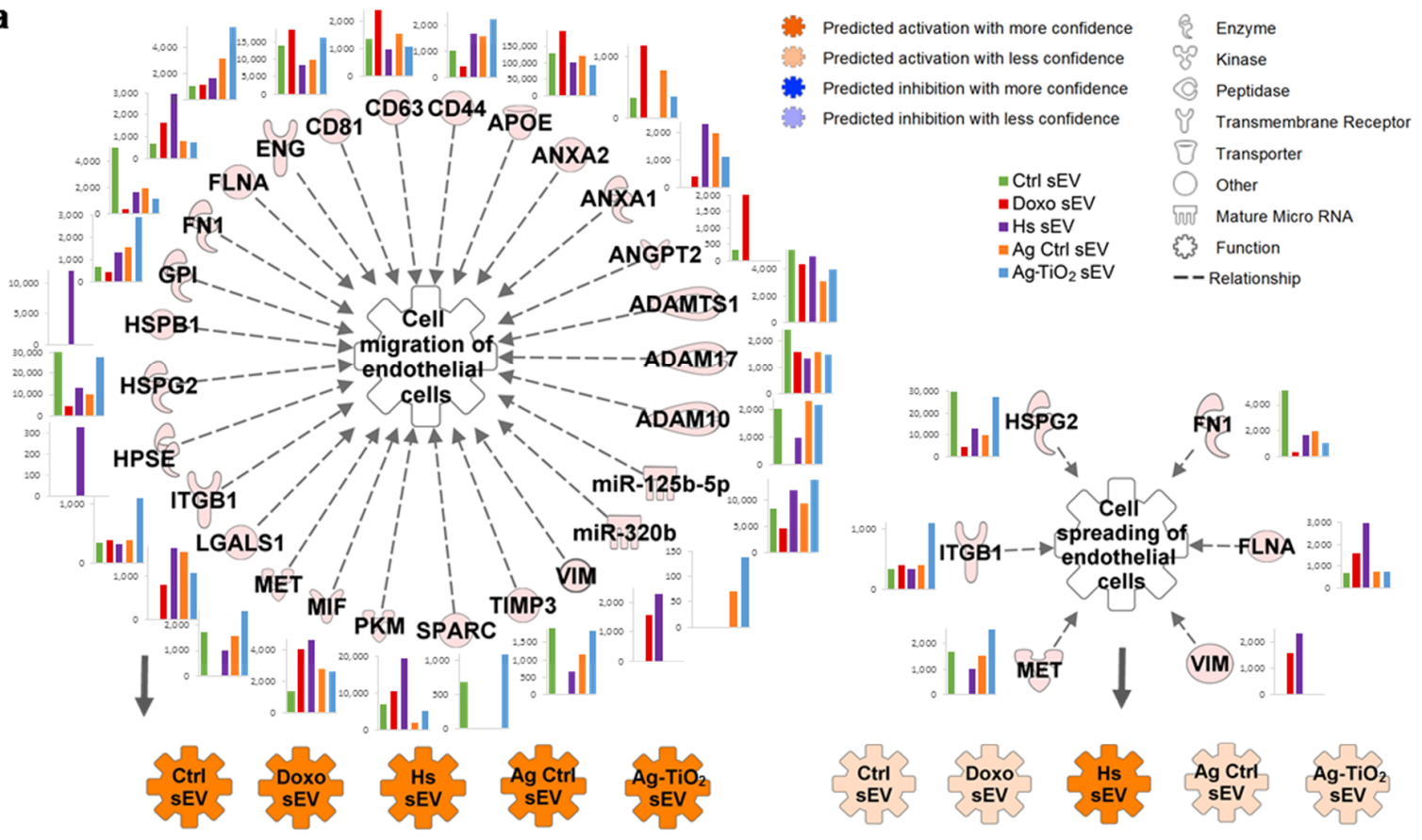

b
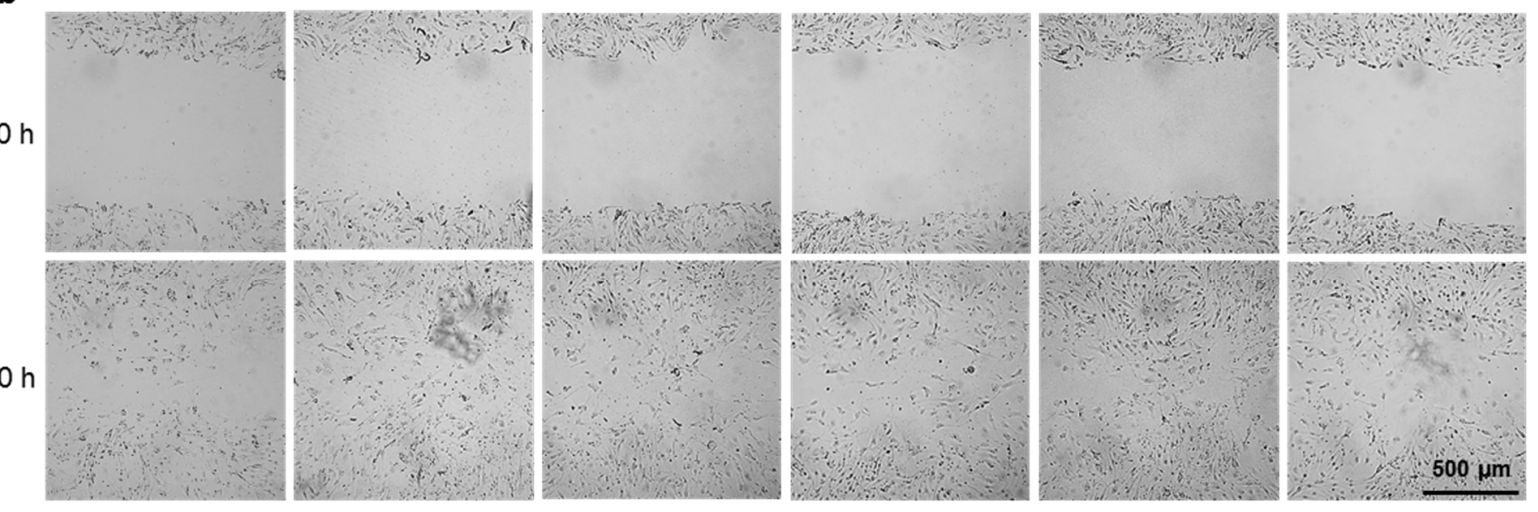

C

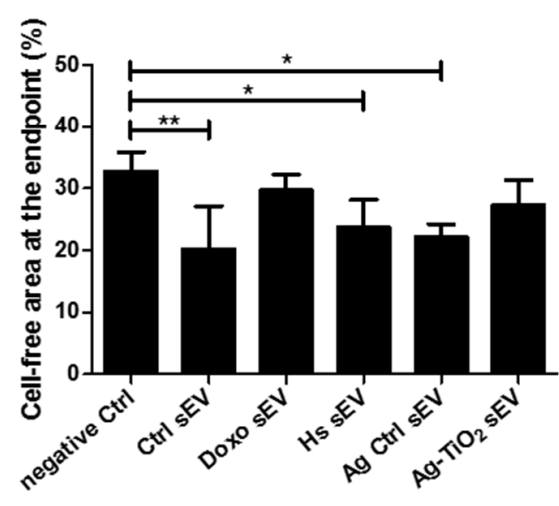

Supplementary Figure S5. Melanoma sEVs facilitate the endothelial cell migration. (a) Networks show the IPA predictions for the regulatory effects of $\mathrm{sEV}$ molecules on the 'Cell migration of endothelial cells' and ,Cell spreading of endothelial cells'. Network shows every upstream regulator proteins and miRNAs accompanied by a bar graph, which represents the normalised expression values of the molecule for each sEV group. Coloured symbols named as the $\mathrm{sEV}$ groups display the expected regulation changes of the analysed 'Biofunctions' upon exposure to the vesicles. (b) Images represent the wounds after $30 \mathrm{~h}$ of sEV exposures. (c) Bar graph shows the result of the analysis of wound closures by the ImageJ wound healing tool. It shows mean+SD values $(n=4)$. 


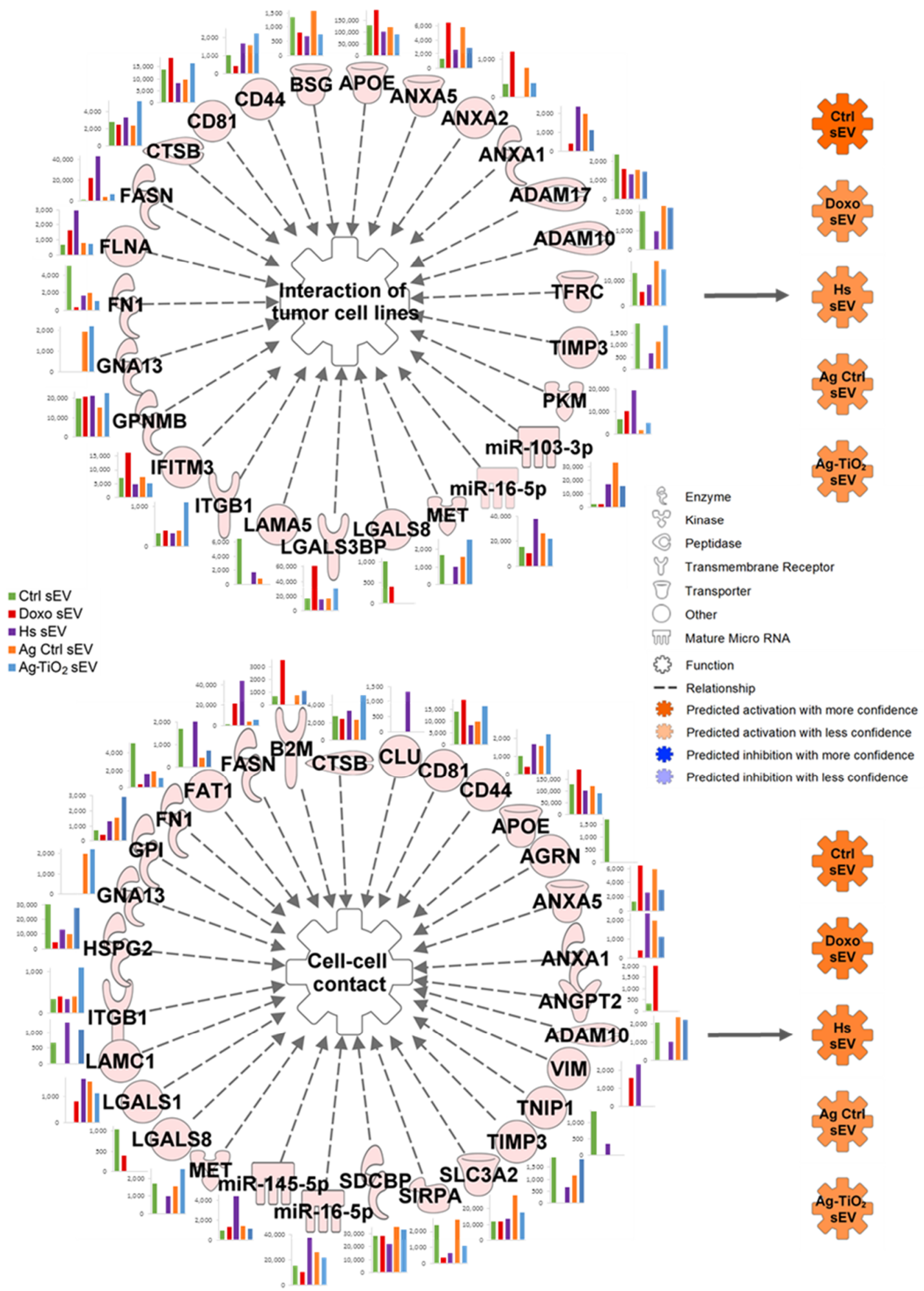

Supplementary Figure S6. IPA predictions for regulatory effect of sEV molecules on the 'Interaction of tumour cell lines' and the 'Cell-cell contact' showed activations. Networks show every upstream regulator proteins and miRNAs accompanied by a bar graph, which represents the normalised expression values of the molecule for each sEV group. Coloured symbols named as the sEV groups display the expected regulation changes of the analysed 'Biofunctions' upon exposure to the vesicles. 


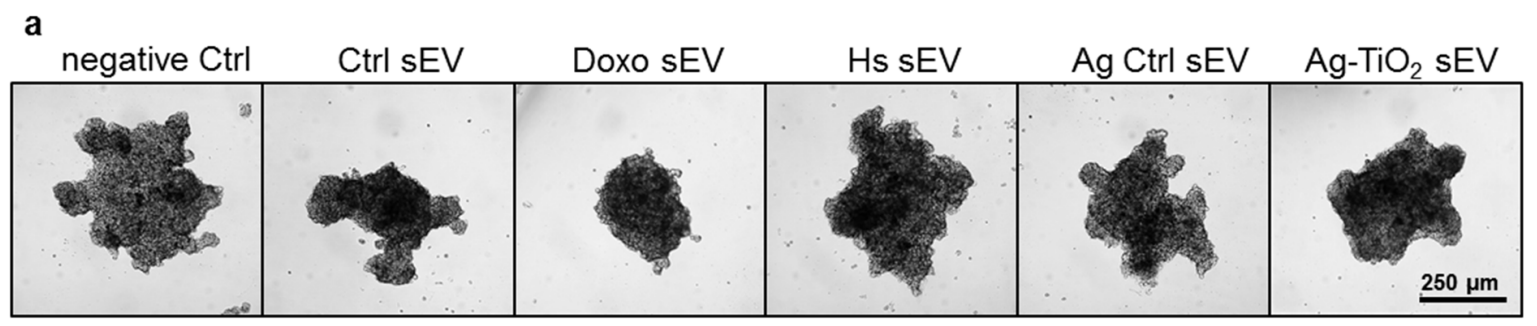

b

\begin{tabular}{|l|c|c|c|c|c|c|}
\hline Group name & negative Ctrl & Ctrl sEV & Doxo sEV & Hs sEV & Ag Ctrl sEV & Ag- TiO $_{2}$ sEV \\
\hline Eq. diameter $(\mu \mathrm{m})$ & $437 \pm 9$ & $351 \pm 15$ & $309 \pm 16$ & $423 \pm 5$ & $347 \pm 18$ & $348 \pm 17$ \\
\hline Major diameter $(\mu \mathrm{m})$ & $519 \pm 17$ & $489 \pm 20$ & $388 \pm 23$ & $530 \pm 20$ & $448 \pm 30$ & $432 \pm 32$ \\
\hline Minor diameter $(\mu \mathrm{m})$ & $323 \pm 12$ & $170 \pm 40$ & $242 \pm 17$ & $306 \pm 19$ & $212 \pm 20$ & $257 \pm 30$ \\
\hline Convexity & $0.662 \pm 0.037$ & $0.632 \pm 0.017$ & $0.730 \pm 0.064$ & $0.585 \pm 0.050$ & $0.690 \pm 0.165$ & $0.630 \pm 0.069$ \\
\hline Solidity & $0.774 \pm 0.021$ & $0.734 \pm 0.011$ & $0.838 \pm 0.009$ & $0.800 \pm 0.020$ & $0.825 \pm 0.080$ & $0.833 \pm 0.046$ \\
\hline Sphericity & $0.554 \pm 0.042$ & $0.501 \pm 0.013$ & $0.629 \pm 0.042$ & $0.491 \pm 0.034$ & $0.601 \pm 0.180$ & $0.547 \pm 0.066$ \\
\hline
\end{tabular}
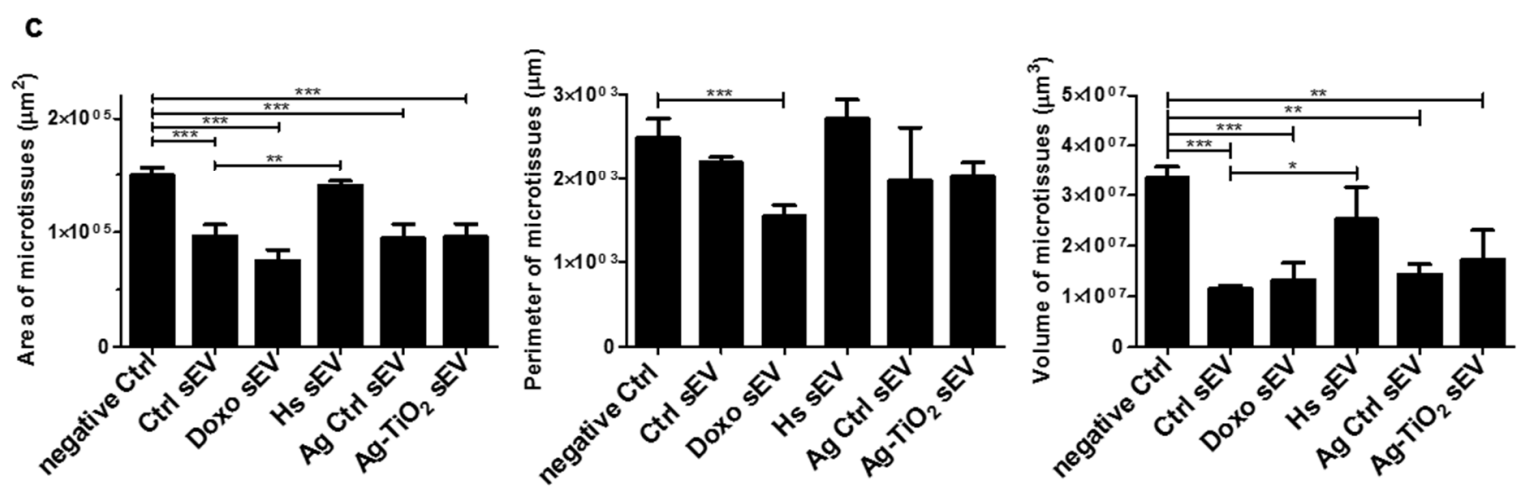

Supplementary Figure S7. Validation of the microtissue generation promoting effects of sEVs using MEFB16F1 3D co-cultures. (a) Images represent the generated microtissues after $72 \mathrm{~h}$ of sEV exposures. (b) Table shows the statistics about the calculated diameters and compactness using the AnaSP software. Eq. diameter means equivalent diameter, major and minor diameter is measured through centroid. Table contains mean $\pm \mathrm{SD}$ values. (c) Bar graphs show the area, perimeter and volume statistics of the generated microtissues (mean+SD, $\mathrm{n}=3$ ). Statistical evaluation was performed by Welch's ANOVA test with Tukey' HSD post-hoc test; *p $<0.05$, $* * \mathrm{p}<0.01$ and $* * * \mathrm{p}<0.001$ indicate statistical significance. 


\section{Supplementary Methods}

\section{Proteomic analysis by LC-MS/MS}

\section{Separation and in-gel digestion of $S E V$ proteins}

Vesicular pellets were resuspended in NP40 lysis buffer (Invitrogen) and incubated on ice for $30 \mathrm{~min}$. Protein content of sEV isolates were measured as described above and $25 \mu \mathrm{g}$ protein of each sample was diluted in a mixture of $4 \times$ sample buffer (NuPAGE LDS Sample Buffer) and $10 \times$ reducing agent (NuPAGE Sample Reducing Agent), boiled at $96^{\circ} \mathrm{C}$ for $10 \mathrm{~min}$, immediately cooled on ice and separated in 4-12\% Bis-Tris Protein Gel (NuPAGE Novex) using an XCell SureLock Mini-Cell (Invitrogen) at $200 \mathrm{~V}$ and $0.03 \mathrm{~A}$ for 40 min with an electrophoresis buffer (NuPAGE MOPS SDS Running Buffer). Then the gel was stained overnight with Coomassie blue (0.1\% Coomassie Brilliant Blue R-250 in 45\% ethanol, $45 \%$ MQ water and $10 \%$ acetic acid), destained with a mixture of $10 \%$ acetic acid, $30 \%$ ethanol and $60 \%$ MQ water and washed in MQ water. Each lane was cut to 12 equal bands and subjected to in-gel digestion. Gel bands were diced to smaller pieces, and the SDS and the dye were washed out with $3 \times 50 \mu 125 \mathrm{mM}$ ammonium-bicarbonate $(\mathrm{ABC}) / 50 \%$ Acetonitrile $(\mathrm{AcN})$. After reduction with DTT (1,4-dithiothreitol, Sigma; $20 \mu 1,10 \mathrm{mM}$ DTT in $25 \mathrm{mM} \mathrm{ABC}$ ) at $56^{\circ} \mathrm{C}$ for $30 \mathrm{~min}$, and alkylation with IAM (iodoacetamide, Sigma; $20 \mu 1,55 \mathrm{mM}$ IAM in $25 \mathrm{mM} \mathrm{ABC}$ ) at room temperature (RT) in dark for $30 \mathrm{~min}$, the gel samples were dried in a vacuum centrifuge and after that rehydrated in $20 \mu 1$ of trypsin (Sequencing Grade Modified Trypsin, Promega; $5 \mathrm{ng} / \mu 1$ in $25 \mathrm{mM} \mathrm{ABC}$ ) and incubated at $37^{\circ} \mathrm{C}$. The digestion was stopped after $4 \mathrm{~h}$ by lowering the $\mathrm{pH}$ of the buffer below 3, by adding $2 \mu 1$ of $10 \%$ formic acid (FA). Tryptic peptides were extracted from the gel with $3 \times 50 \mu 1$ of $2 \%$ FA in $50 \% \mathrm{AcN}$ and dried. Prior mass spectrometric analysis, all samples were redissolved in $50 \mu 1$ of $0.1 \%$ FA.

\section{Mass spectrometry analysis and protein identification}

Samples were analysed on an LTQ-Orbitrap Elite (Thermo Scientific) mass spectrometer online coupled with a nanoHPLC (nanoAcquity, Waters) system. Five $\mu l$ of the in-gel digests were loaded (for $3 \mathrm{~min}$ at $8 \mu \mathrm{l} / \mathrm{min}$ flow, using $0.1 \% \mathrm{FA}$ in 3\% AcN-97\% water) onto a reversed phase trap column (Waters, Symmetry C18, 0.18x20 mm) and separated on a C18 reversed phase (Waters, BEH300C18 $1.7 \mu \mathrm{m})$ nanocolumn $(0.075 \times 200 \mathrm{~mm})$. The flow rate was 
$330 \mathrm{nl} / \mathrm{min}$ and a linear gradient was used from $3 \%$ to $40 \%$ solvent B in $37 \mathrm{~min}$ (solvent A was $0.1 \% \mathrm{FA}$ in water and solvent $\mathrm{B}$ was $0.1 \% \mathrm{FA}$ in $\mathrm{AcN}$ ).

The high voltage $(1.2 \mathrm{kV})$ was applied through the liquid junction between the chromatographic column and the non-coated silica nanospray emitter (NewObjective, $10 \mu \mathrm{m}$ tip ID). The mass spectrometer operated in data-dependent mode: the survey mass spectra were detected in the Orbitrap with high resolution ( $\mathrm{R}=60 \mathrm{k} @ \mathrm{~m} / \mathrm{z}: 400$, mass range m/z: 380-1400) and the most abundant multiply charged 20 peaks were selected for ion-trap fragmentation (NCE: 35\%; activation q: 0.25 ; activation time: $10 \mathrm{~ms}$; minimum signal intensity: 5000 counts). The MS/MS spectra were detected in the ion trap. Dynamic exclusion was used, the precursors were excluded for $15 \mathrm{~s}$ after the first fragmentation event.

Data analysis: searchable peaklists (mgf format) were extracted using Proteome Discoverer 1.4 (Thermo Scientific) and subjected to database search on our in-house ProteinProspector 5.14.1 search engine using the following parameters: parent ion tolerant: $5 \mathrm{ppm}$; fragment ion tolerant: 0.6 Da; Cys carbamidomethylation was set as constant and Met oxidation, cyclisation of peptide N-terminal Glu to pyroglutamic acid, protein $\mathrm{N}$ terminal acetylation were set as variable modifications. Only fully tryptic peptides were considered with maximum of 2 missed cleavage sites. The Mus musculus and Bos taurus protein sequences of the Uniprot (UniProtKB.06.11.2014) database completed with human keratins and pig trypsin, altogether 106,330 protein sequences were searched. For the FDR estimation, the searches were performed on the database concatenated with the randomized sequences. Protein identification was accepted if the ProteinProspector expectation value was less than 0.01 and the protein was identified with at least 3 unique peptides (expectation value less than 0.05 and score higher than 15). Peptides with identical bovine and mouse sequence were excluded also. FDR values were less than $1 \%$ in all cases. 
I. 


\title{
SCIENTIFIC REPORTS
}

\section{Small extracellular vesicles convey the stress-induced adaptive responses of melanoma cells}

\author{
Maria Harmati ${ }^{1,2}$, Edina Gyukity-Sebestyen ${ }^{1,2}$, Gabriella Dobra ${ }^{1,2}$, Laszlo Janovak ${ }^{3}$, \\ Imre Dekany ${ }^{3}$, Okay Saydam ${ }^{4}$, Eva Hunyadi-Gulyas $\mathbb{1 0}^{5}$, Istvan Nagy ${ }^{6}{ }^{6}$, Attila Farkas ${ }^{7}$, \\ Tibor Pankotai ${ }^{8,9}$, Zsuzsanna Ujfaludi ${ }^{8,9}$, Peter Horvath ${ }^{1,10}$, Filippo Piccinini ${ }^{11}$, Maria Kovacs ${ }^{1}$, \\ Tamas Biro ${ }^{12,13}$ \& Krisztina Buzas ${ }^{1,9 *}$
}

Exosomes are small extracellular vesicles (sEVs), playing a crucial role in the intercellular communication in physiological as well as pathological processes. Here, we aimed to study whether the melanoma-derived sEV-mediated communication could adapt to microenvironmental stresses. We compared B16F1 cell-derived sEVs released under normal and stress conditions, including cytostatic, heat and oxidative stress. The miRNome and proteome showed substantial differences across the SEV groups and bioinformatics analysis of the obtained data by the Ingenuity Pathway Analysis also revealed significant functional differences. The in silico predicted functional alterations of $s E V s$ were validated by in vitro assays. For instance, melanoma-derived $\mathrm{sEVs}$ elicited by oxidative stress increased $\mathrm{Ki}-67$ expression of mesenchymal stem cells (MSCs); cytostatic stress-resulted sEVs facilitated melanoma cell migration; all SEV groups supported microtissue generation of MSC-B16F1 co-cultures in a 3D tumour matrix model. Based on this study, we concluded that (i) molecular patterns of tumourderived $\mathrm{sEVs}$, dictated by the microenvironmental conditions, resulted in specific response patterns in the recipient cells; (ii) in silico analyses could be useful tools to predict different stress responses; (iii) alteration of the $s E V$-mediated communication of tumour cells might be a therapy-induced host response, with a potential influence on treatment efficacy.

Exosomes are small $(30-200 \mathrm{~nm})^{1}$ endosome-derived vesicles that are actively secreted into the extracellular environment from most cell types. Initially, exosomes were proposed to eliminate cellular waste, but it has been proven that they also play a key role in the intercellular communication between adjacent as well as distal cells through the horizontal transfer of lipids, proteins and nucleic acids. Over the past three decades, exosomes have surged to the forefront of cell biology research, and recently an increasing body of evidence indicates that this exosomal communication is a deliberate and highly orchestrated process. Clinical relevance of exosomes is also considerable, since they are associated with numerous physiological and pathological conditions, including cancer diseases ${ }^{2}$.

\footnotetext{
${ }^{1}$ Laboratory of Microscopic Image Analysis and Machine Learning, Institute of Biochemistry, Biological Research Centre of the Hungarian Academy of Sciences, Szeged, Hungary. ${ }^{2}$ Doctoral School of Interdisciplinary Medicine, Faculty of Medicine, University of Szeged, Szeged, Hungary. ${ }^{3}$ Interdisciplinary Excellence Centre, Department of Physical Chemistry and Materials Science, University of Szeged, Szeged, Hungary. ${ }^{4}$ Department of Pediatrics, University of Minnesota, Minneapolis, USA. ${ }^{5}$ Laboratory of Proteomics Research, Institute of Biochemistry, Biological Research Centre of the Hungarian Academy of Sciences, Szeged, Hungary. ${ }^{6}$ Sequencing Platform, Institute of Biochemistry, Biological Research Centre of the Hungarian Academy of Sciences, Szeged, Hungary. ${ }^{7}$ Laboratory of Microbial Genomics, Institute of Plant Biology, Biological Research Centre of the Hungarian Academy of Sciences, Szeged, Hungary. ${ }^{8}$ Department of Biochemistry and Molecular Biology, Faculty of Science and Informatics, University of Szeged, Szeged, Hungary. ${ }^{9}$ Department of Oral Biology and Experimental Dental Research, Faculty of Dentistry, University of Szeged, Szeged, Hungary. ${ }^{10}$ Institute for Molecular Medicine Finland, University of Helsinki, Helsinki, Finland. ${ }^{11}$ Laboratorio di Bioscienze, Istituto Scientifico Romagnolo per lo Studio e la Cura dei Tumori (IRST) IRCCS, Meldola, Italy. ${ }^{12}$ Department of Immunology, Faculty of Medicine, University of Debrecen, Debrecen, Hungary. ${ }^{13}$ Hungarian Centre of Excellence for Molecular Medicine, Szeged, Hungary. *email: kr.buzas@gmail.com
} 


\begin{tabular}{|l|l|l|l|l|l|}
\hline Conditions & Control 1 & Cytostatic stress & Heat stress & Control 2 & Oxidative stress \\
\hline sEV donor cell cultures & Ctrl & Doxo & $\mathrm{Hs}$ & $\mathrm{Ag}$ Ctrl & $\mathrm{Ag}^{-\mathrm{TiO}_{2}}$ \\
\hline Treatment & - & $0.6 \mu \mathrm{M}$ doxorubicin & $3 \times 2 \mathrm{~h}$ at $42^{\circ} \mathrm{C}$ & illuminated medium & $\begin{array}{l}\text { light-induced } \\
2.5 \mu \mathrm{gg} / \mathrm{ml} \mathrm{Ag}-\mathrm{TiO} \\
2\end{array}$ \\
\hline Released sEV groups & Ctrl sEV & Doxo sEV & $\mathrm{Hs} \mathrm{sEV}$ & $\mathrm{Ag} \mathrm{Ctrl} \mathrm{sEV}$ & $\mathrm{Ag}-\mathrm{TiO}_{2} \mathrm{sEV}$ \\
\hline
\end{tabular}

Table 1. Treatment schedule of tumour cell cultures and the isolated sEV groups.

Tumours are not just insular masses of proliferating cancer cells; they are also complex tissues composed of cellular components, such as mesenchymal stem cells (MSCs), cancer-associated fibroblasts (CAFs), endothelial cells, immune cells as well as extracellular matrix (ECM) components, which establish the so-called tumour microenvironment (TME) surrounding the tumour cells. The TME does not only surround the tumour cells, it also actively contributes to tumour progression, which requires a continuous paracrine communication ${ }^{3,4}$. One of the possible candidates for intercellular communication might be exosomes, since several recent papers have emphasized the mediating role of exosomes in the tumour macro- and microenvironment ${ }^{4-6}$. Upon contact with recipient cells, tumour-derived exosomes alter their phenotypic and functional properties conveying molecular and genetic messages ${ }^{7}$.

Since malignant melanoma is one of the most aggressive cancers, the B16F1 mouse melanoma cell line was chosen as a tumour cell model for this study. Melanoma cells potentially disseminate from a relatively small primary tumour and form metastases in multiple sites, including the lung, liver, brain, bone and lymph nodes ${ }^{8}$. This high metastatic potential can be explained by the unique features of melanoma. For instance, melanoma cells are mesenchymal in nature ensuring that a larger percentage of cells can act as stem cells with self-renewal capacity. They share many antigens with vascular endothelial cells (vasculogenic mimicry) which enables them to survive in the circulation, and increases their migration and invasion capacity as well. Furthermore, melanoma-derived extracellular vesicles also have a crucial role in the rapid tumour progression ${ }^{8-12}$. They are capable to induce a tumour-favourable phenotype in the EV-recipient cells in the $\mathrm{TME}^{13}$ and the metastatic sites ${ }^{14,15}$. For instance, after re-education by exosomes, MSCs may promote tumour growth and metastasis ${ }^{16,17}$; fibroblast ${ }^{18}$ and endothelial cells ${ }^{19}$ can promote angiogenesis. There are also a few papers about intrinsic stresses, such as low $\mathrm{pH}^{20}$ - or hypoxia $^{21}$-induced alterations of melanoma exosomes, and their ability to transfer drug resistance ${ }^{22}$. However, different extrinsic stress-elicited changes have not been elucidated yet.

Using a unique approach, this study compared changes in the vesicular information transfer of melanoma cells under different inducible stress conditions. Exosomes are complex information packages, and we consider them as message delivering units with specific molecular patterns, rather than putting emphasis on a few exosomal signal molecules. Following the guideline recommended by ISEV (International Society for Extracellular Vesicles), called MISEV2018 (Minimal information for studies of extracellular vesicles 2018) ${ }^{23}$, we refer to the isolated vesicles based on their size by using the term 'small extracellular vesicles' (sEVs), even though their exosomal characteristics are demonstrated.

To gain insights into the plasticity and role of sEVs under suboptimal conditions, we investigated cytostatic, heat and oxidative stress-induced alterations of the B16F1 mouse melanoma cell-derived sEVs. Clinical relevance of this study is highlighted by previous papers, since chemo- or radiotherapy were shown to increase the amount of circulating tumour-derived $\mathrm{EV} \mathrm{s}^{24,25}$. Although chemotherapy provides long-term clinical benefits to patients, it may induce tumour-promoting host responses as well ${ }^{26}$. Furthermore, Keklikoglou et al. have shown that paclitaxel and doxorubicin elicit the production of pro-metastatic breast cancer-derived EVs ${ }^{27}$. Hyperthermia treatment involves increasing the target site temperature to induce thermic stress, which results in cancer cell cytotoxicity and immune response stimulation via immune cell activation. Therefore, hyperthermia may enhance the therapeutic efficacy in combined therapies ${ }^{28,29}$, but its impact on tumour exosome-mediated intercellular communication has not been described yet. To induce oxidative stress, we used $\mathrm{Ag}-\mathrm{TiO}_{2}$ photocatalyst particles, which represent a high potential for therapeutic applications through antibacterial ${ }^{30,31}$, antifungal ${ }^{32}$ and antican$\mathrm{cer}^{33}$ activities. However, the effects of $\mathrm{Ag}^{-\mathrm{TiO}_{2}}$-based therapies on the vesicular communication have not been investigated yet.

\section{Results and Discussion}

In order to study the adaptive sEV-mediated communication under microenvironmental stress, we investigated cytostatic, heat and oxidative stress-induced alterations of the B16F1 mouse melanoma cell-derived sEVs. After verification the exosomal characteristics of our $\mathrm{sEV}$ isolates, we optimized the cytostatic and oxidative stress treatment conditions by proliferation assay and established the protocol for heat stress based on literature data $^{34}$ to expose the melanoma cells to sublethal stress conditions. Then, for the sEV production, we cultured the B16F1 cells under five different conditions in EV-depleted FBS-containing media; control cultures (Ctrl) received culture medium, cytostatic stressed cultures (Doxo) were treated with $0.6 \mu \mathrm{M}$ doxorubicin, heat stressed cultures (Hs) were incubated at $42^{\circ} \mathrm{C}$ for $3 \times 2 \mathrm{~h}$, oxidative stressed cultures $\left(\mathrm{Ag}-\mathrm{TiO}_{2}\right)$ were treated with $2.5 \mu \mathrm{g} / \mathrm{ml}$ light-induced $\mathrm{Ag}-\mathrm{TiO}_{2}$, and as a control of the oxidative stress (Ag Ctrl), additional cultures were treated with illuminated media (Table 1). After a $72 \mathrm{~h}$ stress exposure period of B16F1 cultures, sEVs were isolated from their supernatants, quantified by nanoparticle tracking analysis (NTA) and analysed by SOLiD sequencing and LC-MS/MS to determine the miRNome and proteome of sEVs. Functional differences between sEV groups were predicted first in silico using the Ingenuity Pathway Analysis (IPA) based on the protein and miRNA data, and then verified by in vitro experiments targeting tumour-related cellular functions, such as Ki-67 expression, cell cycle dynamics, migration capacity and microtissue generation of the recipient cells (Fig. 1). 
Our oxidative stress model is based on the photocatalytic activity of the $\mathrm{Ag}-\mathrm{TiO}_{2}$ particles ${ }^{31,35}$. During the process of photocatalysis under appropriate (exciting) wavelength, reactive hydroxyl radicals $(\mathrm{OH} \cdot)$ are produced, which are primarily responsible for photooxidation of organic materials or inactivating bacteria ${ }^{36}$. Hydroxyl radicals are the most reactive oxygen species and cause irreversible DNA damages which could lead to DNA degradation in bacteria ${ }^{36}$. In our previous work, the amount of reactive hydroxyl radicals formed on $\mathrm{Ag}-\mathrm{TiO}_{2}$ particles was determined by the hydrogen peroxide-induced luminol-dependent chemiluminescence reaction ${ }^{30}$. It was presented that concentration of the $\mathrm{Ag}-\mathrm{TiO}_{2}$-produced $\mathrm{OH}$. radicals was equivalent to $0.33 \mathrm{mM} \mathrm{H}_{2} \mathrm{O}_{2}$ after 20 min visible light illumination.

Descriptive statistics of sEVs released under different microenvironmental conditions. Isolated EVs fulfil the minimal experimental requirements for small extracellular vesicles (sEVs). First, to fulfil the minimal experimental requirements for extracellular vesicles, suggested in the MISEV2018 ${ }^{23}$, we characterised the $\mathrm{B} 16 \mathrm{~F} 1$ cell-derived extracellular vesicles isolated from conditioned media by differential filtration and ultracentrifugation. Presence of the vesicles in the sEV isolates was verified by atomic force microscopy (AFM), and size distribution of the isolated vesicle population was described by dynamic light scattering (DLS) with a Z-average of $78 \mathrm{~nm}$. EV markers, such as CD63 and CD9 (transmembrane proteins), HSP70, Alix and TSG101 (cytosolic proteins), Calnexin (negative sEV marker) were investigated in the vesicle isolates and the donor cell lysates by Western blot (Supplementary Fig. S1).

Vesicle production of melanoma cells is elevated under stress conditions. Scanning electron microscopy (SEM) revealed spectacular morphological changes of the $\mathrm{B} 16 \mathrm{~F} 1$ cells in each stressed group (Doxo, $\mathrm{Hs}$ and $\mathrm{Ag}-\mathrm{TiO}_{2}$ ) $24 \mathrm{~h}$ after treatments (Fig. 2a, top panels). Taking advantage of the high magnification capacity of SEM, we were able to observe the surface structures of the cells as well (Fig. 2a, bottom panels). At a 20,000 $\times$ magnification, we discovered spherical, exosome-sized vesicles, which were present in higher numbers on the stressed cells compared to the untreated Ctrl cells ( $\mathrm{p}_{\text {Doxo }}=0.00297, \mathrm{p}_{\mathrm{Hs}}=0.03928, \mathrm{n}=5$; Fig. $2 \mathrm{~b}$ ).

Then, we isolated sEV from conditioned media of the five groups of cell cultures and quantified by the NTA-based NanoSight Analysis. There was a significant increase in vesicle number per cell in the Doxo $\left(20.2 \pm 0.4 \times 10^{3} ; \mathrm{p}=0.00021\right)$ and the Hs $\left(12.7 \pm 3.8 \times 10^{3} ; \mathrm{p}=0.03006\right)$ groups compared to the Ctrl one $\left(5.35 \pm 0.7 \times 10^{3}\right)$. The $\mathrm{Ag}-\mathrm{TiO}_{2}$-treated cultures produced $13 \pm 1.8 \times 10^{3} \mathrm{sEV}$ s per cell, while production of the Ag Ctrl group was only $7.9 \pm 1.6 \times 10^{3} \mathrm{sEVs}$ per cell $(\mathrm{n}=3$, Fig. $2 \mathrm{c})$. At the same time, stress conditions did not affect the size distribution of sEVs.

Other reports also demonstrated that cells, including tumour cells, release a higher amount of exosomes in response to different types of stresses ${ }^{37}$, such as hypoxia ${ }^{38}$, acidosis ${ }^{39}$, oxidative stress ${ }^{40}$, thermal stress ${ }^{40}$, radiation $^{41}$ and cytotoxic drugs ${ }^{42-44}$.

Concentration of the encapsulated doxorubicin under cytostatic stress is less than $10 \%$ of median lethal dose. To reveal if doxorubicin, used for cytostatic stress in $0.6 \mu \mathrm{M}$ concentration, could be encapsulated into the vesicles, Doxo sEV isolates were analysed by fluorescence spectroscopy. Using the Ctrl sEVs as a background, the calculated doxorubicin concentration of the Doxo sEVs was $14.735 \mathrm{nM}$.

Based on the measurements, the Doxo sEV suspensions - used for treatment of recipient cells - contained only $8 \mathrm{ng} / \mathrm{ml}$ doxorubicin. Therefore, it cannot be excluded that sEVs may transfer doxorubicin to the recipient cells, but the doxorubicin content of Doxo sEVs is less than $10 \%$ of the median lethal dose (LD50 $=100 \mathrm{ng} / \mathrm{ml})$ for mouse melanoma cells ${ }^{45}$.

Encapsulation of $\mathrm{Ag}-\mathrm{TiO}_{2}$ nanoparticles into sEVs cannot be proven. To investigate the possibility of $\mathrm{Ag}-\mathrm{TiO} \mathrm{O}_{2}$ encapsulation into sEVs, we measured the size distribution of the nanoparticles by DLS. The mean particle size was around $255 \mathrm{~nm}$ during the whole studied time interval (Supplementary Fig. S2). This means that the initial $\mathrm{Ag}-\mathrm{TiO}_{2}$ photocatalyst particles form aggregates because the primer size of the $\mathrm{Ag}-\mathrm{TiO}_{2}$ particles is around $25 \mathrm{~nm}$ as seen in the TEM image in our previous paper ${ }^{46}$. Thus, DLS measurements did indicate particle aggregation in the used medium; however, it also can be seen that the size of these particle aggregates did not change during the experiments. These results suggest that the $\mathrm{Ag}-\mathrm{TiO}_{2}$ particles cannot be transferred into the recipient cells in this aggregated form and they cannot contaminate the $\mathrm{sEV}$ isolates, since particles over $220 \mathrm{~nm}$ were eliminated during the isolation process.

We also tried to detect disaggregated $\mathrm{Ag}-\mathrm{TiO}_{2}$ nanoparticles in the $\mathrm{sEV}$ isolates using chemiluminescence (CL) method and transmission electron microscopy (TEM). After photoirradiation of the $\mathrm{Ag}_{-} \mathrm{TiO}_{2}$, generated ROS can be detected by adding luminol, which emit light upon oxidation ${ }^{30}$. We compared the CL intensity of $\mathrm{Ag}-\mathrm{TiO}_{2}$ $\mathrm{sEV}$ lysates to an $\mathrm{Ag}-\mathrm{TiO}_{2}$ calibration curve prepared in $\mathrm{Ag}$ Ctrl sEV lysate, but it was under the detection limit $(0.25 \mu \mathrm{g} / \mathrm{ml}$; Supplementary Fig. S2). Moreover, on TEM images we could not observe any internal structures of the sEVs or contaminating electron-dense nanoparticles in the $\mathrm{Ag}-\mathrm{TiO}_{2} \mathrm{sEV}$ isolate (Supplementary Fig. S2).

miRNome and proteome of the melanoma sEVs strongly depend on the microenvironmental conditions of the donor cells. Exosomes deliver a wide range of RNAs and proteins to convey messages to the recipient cells. Their molecular content correlates with the type and state of the donor cell ${ }^{2}$. In this study, we compared the miRNA and protein patterns of the $\mathrm{sEV}$ groups to show the influence of the microenvironment on these information packages.

A total of 254 miRNAs were identified by SOLiD sequencing with more than ten read counts; $35.04 \%$ of these miRNAs were detected in each sEV group, while $6.30 \%, 1.18 \%$ and $0.79 \%$ were exclusively detected in the Ag- $\mathrm{TiO}_{2}$, Ctrl and Doxo sEVs, respectively. Hs and Ag Ctrl sEV-specific miRNAs were not found (Fig. 3a, left panel). The proportion of the over- and underrepresented miRNAs under stress conditions was the highest in the $\mathrm{Ag}-\mathrm{TiO}_{2}$ sEVs, where $53.41 \%$ of the detected miRNAs showed more than twofold changes compared to the 
(1) sEV production

(2) sEV characterisation
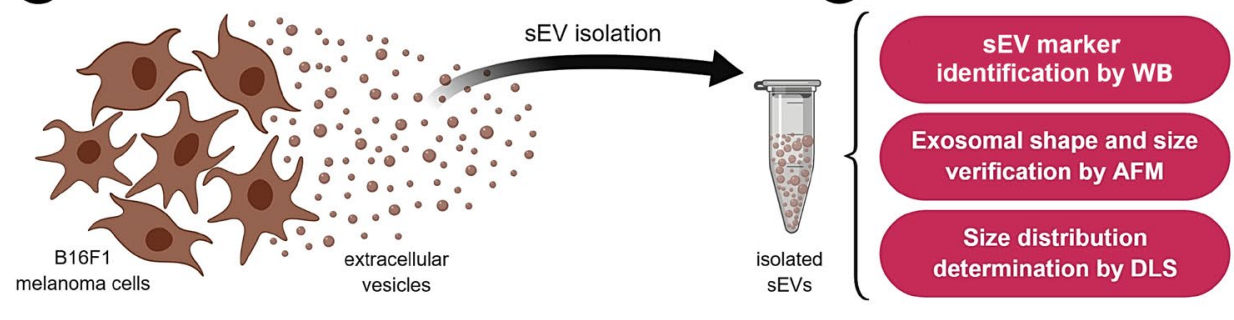

(3) Stress exposures

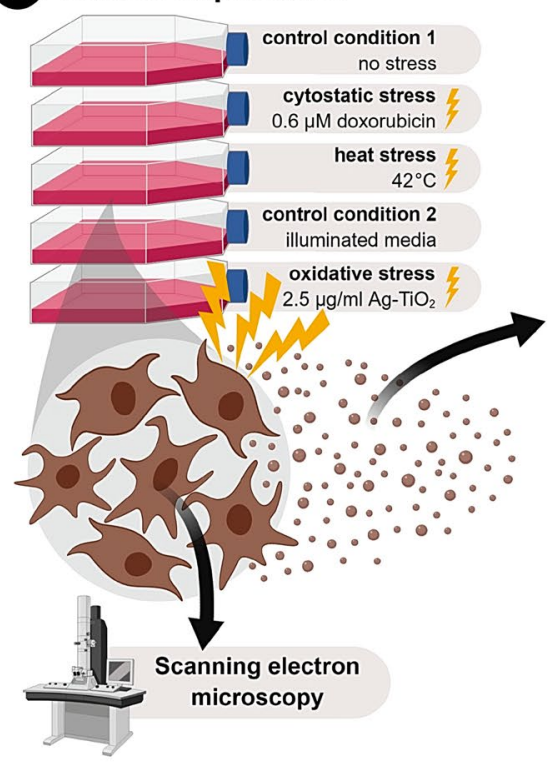

(4) Comparative sEV characterisation

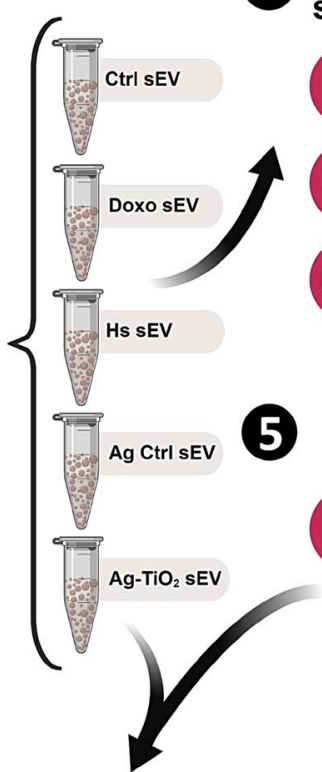

NTA-based quantification

Sequencing of miRNA cargo

LC-MS/MS of protein content

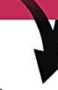

In silico functional analysis of sEVs
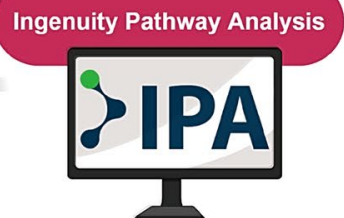

6 In vitro functional studies of sEVs
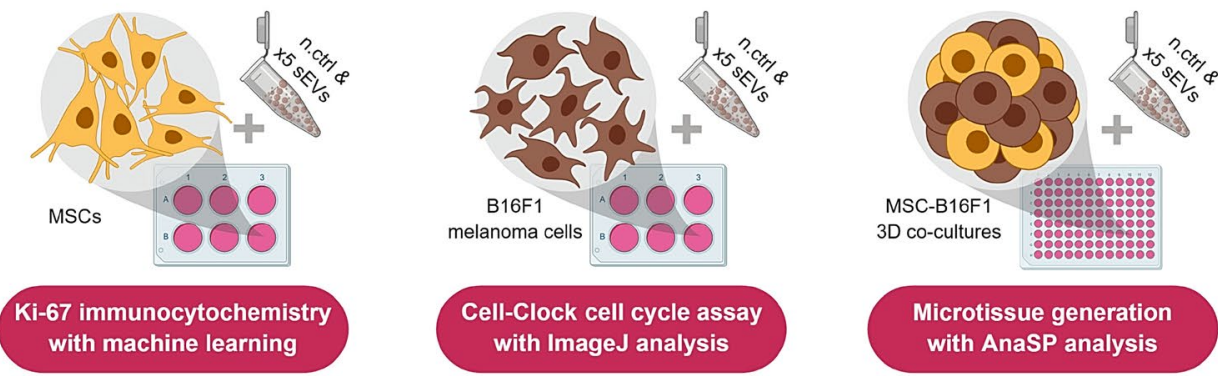

Microtissue generation with AnaSP analysis

Wound healing assay with Image $\mathrm{J}$ analysis

Figure 1. Schematic illustration of the experimental workflow in six steps. B16F1 mouse melanoma cellderived sEVs were isolated and characterised by Western Blot (WB), atomic force microscopy (AFM) and dynamic light scattering (DLS). Then B16F1 cultures were treated in five different ways, and $72 \mathrm{~h}$ supernatants were harvested for sEV isolation. Vesicle samples were then analysed by nanoparticle tracking analysis (NTA) to determine the number of released sEVs, sequencing and LC-MS/MS to describe their miRNome and proteome. Ingenuity Pathway Analysis (IPA) was used to analyse data and predict the functional differences between sEV groups. This in silico predictions were tested in vitro on mesenchymal stem cell (MSC) and melanoma cell cultures and MSC-B16F1 3D co-cultures as well using Ki-67-specific immunocytochemistry, Cell-Clock cell cycle assay, wound healing assay, and 3D hanging drop technology. Abbreviation: n.ctrl-negative control. Figure was created with BioRender.com.

corresponding control (Fig. 3a, right panel). Results of the sequencing data were validated by qPCR on three major miRNAs of this study, i.e. mmu-miR-16-5p, mmu-miR-125b-5p and mmu-miR-29a-3p (Supplementary Fig. S3).

Using LC-MS/MS, a total of 216 proteins were detected with three or more peptides; $59.72 \%$ of these proteins were common to all sEV groups, and only a few unique proteins were found (Fig. 3b, left panel). Eight Hs sEV-specific 
a
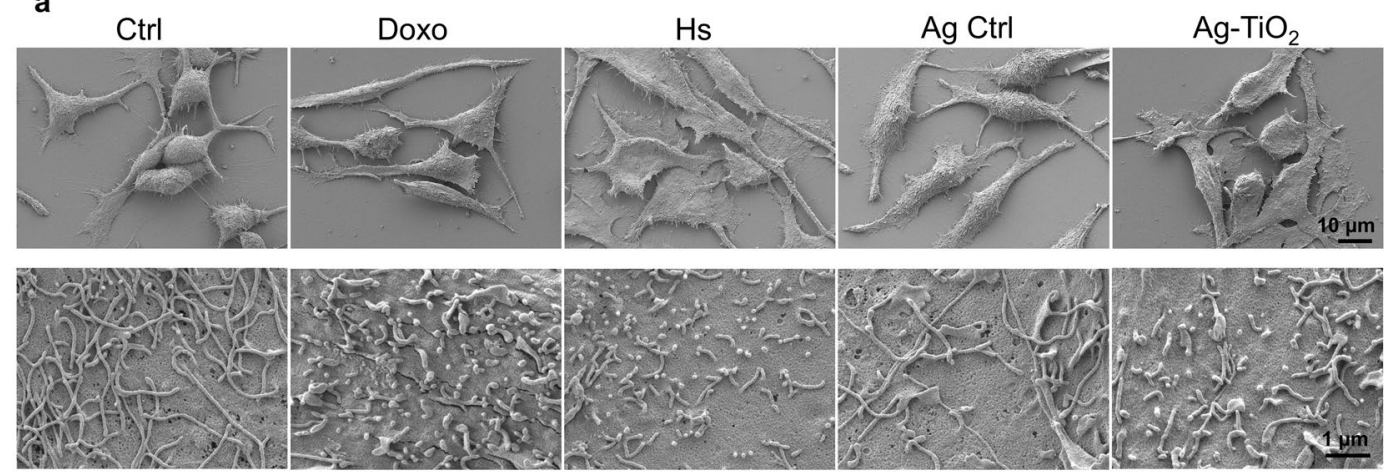

b

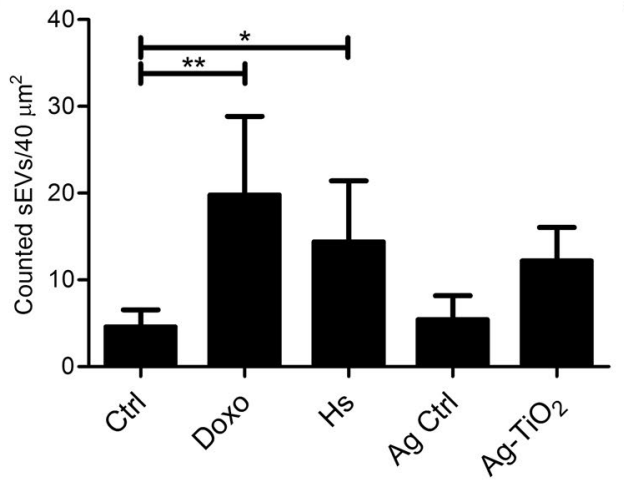

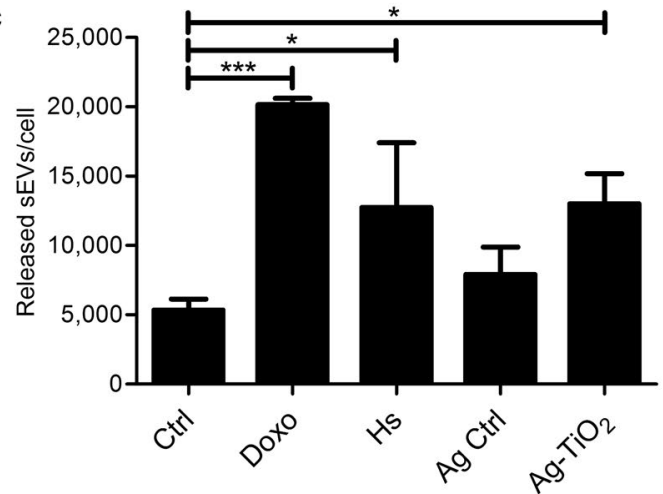

Figure 2. Microenvironmental stress factors resulted in morphological changes and elevated vesicle production of melanoma cells. (a) Scanning electron micrograph of the differently treated melanoma cells. The top row of pictures was taken in 1,500 $\times$ magnification showing the different cell morphology after $24 \mathrm{~h}$ treatments. The bottom row of pictures was taken in 20,000 $\times$ magnification showing the distinct cell surface structures. (b) The number of counted exosome-sized vesicles on the surface of cells using ImageJ $(n=5)$. (c) Number of released vesicles/cell based on NanoSight measurements $(n=3)$. Each bar represents mean $+S D ; * p<0.05, * * p<0.01$ and $* * * \mathrm{p}<0.001$ indicate statistical significance.

proteins were identified; half of these had a chaperone function (HSBP1, SERPINH1, CCT5, CLU). The major impact of heat stress on the protein composition of sEVs was also evidenced by the high proportion of the over- and underrepresented proteins (52.21\%) compared to the Ctrl sEV group. Cytostatic stress also resulted in dramatic and distinct changes in the protein content of vesicles. For instance, the proportion of proteins, which decreased below the detectable level was the highest (15.34\%) in Doxo sEVs (Fig. 3b, right panel). Many of the commonly found proteins have a function in vesicular trafficking, membrane fusion or MVB biology which suggests they have a role in exosome biogenesis. There are also melanocyte-specific molecules, such as some melanin biosynthesis elements (DCT, MLANA, PMEL, TYR or TYRP1), and we found a several metabolism-, cytoskeletal organisation-, extracellular matrix remodelling-related proteins, adhesion molecules, receptors and transporters, which may have a crucial role in tumour progression (Fig. 3c). Proteomics data were validated by Western blot, where a vesicular marker, the HSP70 and the MLANA showed similar signal intensity patterns to the LC-MS/MS data (Supplementary Fig. S4).

Our findings about the molecular cargo of sEVs are in accordance with previous studies, which also showed changes in the exosomal content upon exposure of the donor cells to external stimuli and stress conditions ${ }^{41,44,47,48}$. Furthermore, Peinado et al. ${ }^{17}$ and Lazar et al.$^{49}$, analysing protein composition of different melanoma cell line-derived exosomes, also found melanocyte-specific proteins, transmembrane proteins, such as tetraspanins, transporters and receptors as well as MVB and endosomal pathway-related proteins, for instance ESCRT-associated proteins, annexins, cytoskeletal and small GTP-binding proteins ${ }^{17,49}$.

Comprehensive in silico analysis of functional differences between sEV groups. Since the exosomal cargo is a complex information package containing a large number and wide variety of molecules, it may act on several biological processes in the recipient cells. In this study, we aimed to identify these biological processes even for normal (Ctrl) B16F1 sEVs and also for the stress-exposed cell-derived ones. We performed bioinformatics analyses to interpret the biological context of the obtained miRNA and protein data applying the IPA. This software is based on computer algorithms that analyse the functional connectivity of the molecules using the Ingenuity Knowledge Base. For these in silico analyses, we set the confidence level to 'Experimentally observed' that enables literature data-based analysis, but not unproven predictions. Phrases between apostrophes are 'IPA-specific terms' in this paper.

Using the 'Core analysis' feature, we found a huge overlap in the 'Top 5 canonical pathways' between sEV groups. Namely, 'Gycolysis I', ‘Gluconeogenesis I', 'Eumelanin Biosynthesis' and 'Phagosome maturation' was listed 

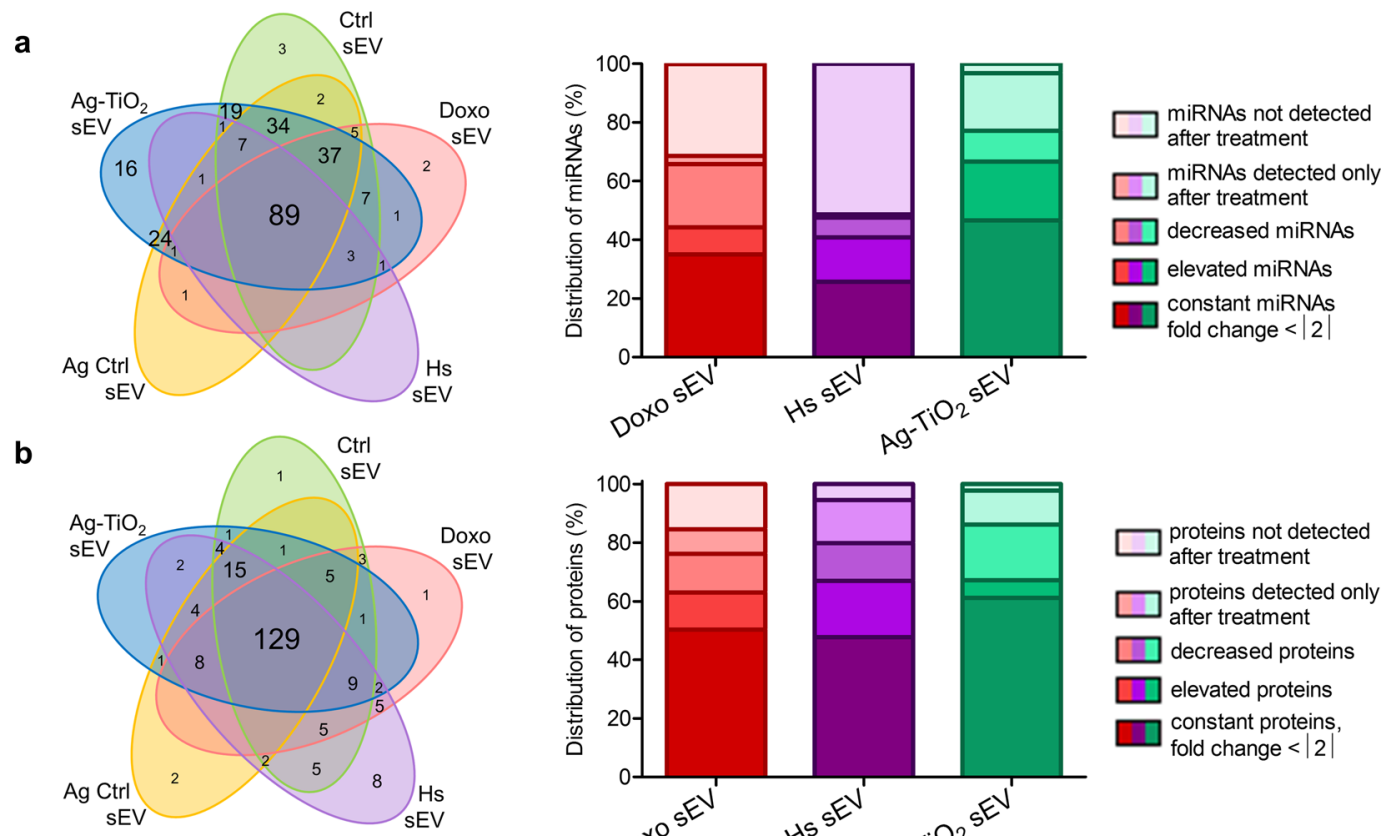

C

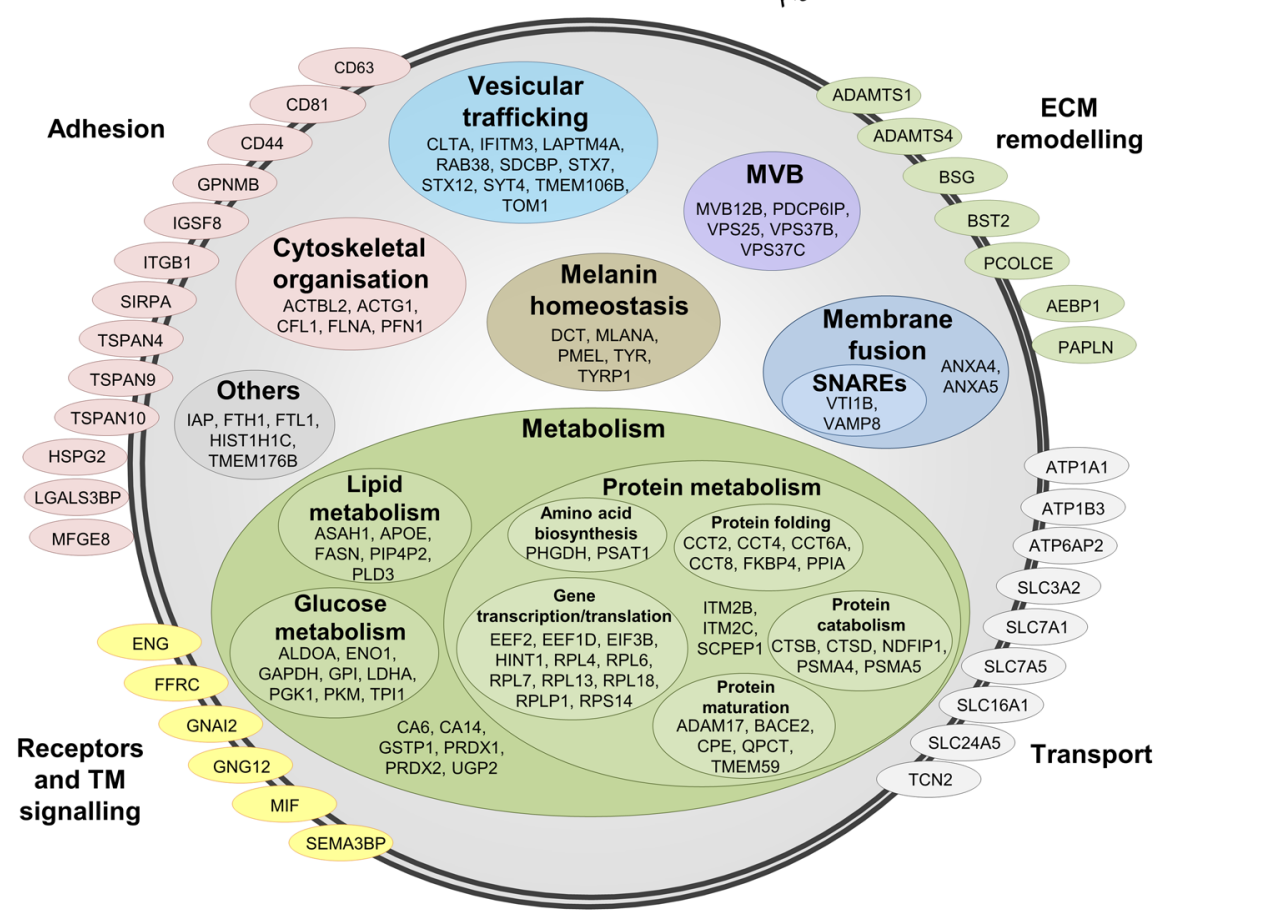

Figure 3. Stress factors caused unique molecular patterns of the melanoma-derived sEVs. (a,b) Results of the miRNA sequencing and whole proteome analysis by LC-MS/MS. Venn diagrams show the number of common and unique molecules of the different sEVs. Stacked bar graphs show the distribution of sEV molecules based on their changes compared to the appropriate control. (c) Classification of the common proteins for each sEV group based on their function and localisation in vesicles.

in all five cases, while 'Inhibition of matrix metalloproteinases' (MMPs) was only found in Ctrl sEVs, and 'EIF2 signalling' was listed in the four other sEV groups (Fig. 4a). As it was shown in the previous section, beside the remarkable differences, sEVs contain many donor cell-specific molecules, which helps to interpret these results. For instance, the Warburg effect, which means, that cancer cells may prefer metabolism via aerobic glycolysis rather than oxidative phosphorylation ${ }^{50,51}$ gives a possible explanation for the strong presence of glycolysis and glyconeogenesis-related molecules in sEVs. Since the investigated B16F1 is a melanin-producing cell line, the reason for the presence of the eumelanin biosynthesis-related molecules is obvious. Vesicular processes of exosome biogenesis $^{52}$ account for the high number of phagosome maturation-related molecules in the isolated vesicles. Presence of the MMP- and EIF2 signalling-related molecules in the sEVs can also be explained by the tumour cell origin ${ }^{53-55}$. 


\begin{tabular}{|l|c|c|c|c|c|}
\hline \multirow{2}{*}{ Top 5 biological pathways } & \multicolumn{5}{c|}{ p-value } \\
\cline { 2 - 7 } & Ctrl sEV & Doxo sEV & Hs sEV & $\begin{array}{c}\text { Ag Ctrl } \\
\text { SEV }\end{array}$ & $\begin{array}{c}\mathrm{Ag}^{-T i O}{ }_{2} \\
\text { SEV }\end{array}$ \\
\hline Glycolysis I & $3.22 \mathrm{E}-08$ & $9.50 \mathrm{E}-09$ & $1.37 \mathrm{E}-08$ & $2.33 \mathrm{E}-08$ & $5.50 \mathrm{E}-08$ \\
\hline Gluconeogenesis I & $1.93 \mathrm{E}-05$ & $8.21 \mathrm{E}-06$ & $1.06 \mathrm{E}-05$ & $1.54 \mathrm{E}-05$ & $2.81 \mathrm{E}-05$ \\
\hline Eumelanin biosynthesis & $2.17 \mathrm{E}-05$ & $1.27 \mathrm{E}-05$ & $1.50 \mathrm{E}-05$ & $1.88 \mathrm{E}-05$ & $2.74 \mathrm{E}-05$ \\
\hline Phagosome maturation & $1.48 \mathrm{E}-04$ & $3.80 \mathrm{E}-05$ & $5.75 \mathrm{E}-05$ & $5.52 \mathrm{E}-04$ & $5.02 \mathrm{E}-05$ \\
\hline $\begin{array}{l}\text { Inhibition of matrix } \\
\text { metalloproteinases }\end{array}$ & $1.64 \mathrm{E}-03$ & $6.79 \mathrm{E}-02$ & $1.04 \mathrm{E}-03$ & $1.38 \mathrm{E}-03$ & $2.18 \mathrm{E}-03$ \\
\hline EIF2 signalling & $1.02 \mathrm{E}-02$ & $4.36 \mathrm{E}-05$ & $4.32 \mathrm{E}-07$ & $1.09 \mathrm{E}-06$ & $4.68 \mathrm{E}-06$ \\
\hline
\end{tabular}

b

\begin{tabular}{|l|c|c|c|c|c|}
\hline \multirow{2}{*}{$\begin{array}{c}\text { Top } 5 \text { molecular and cellular } \\
\text { functions }\end{array}$} & \multicolumn{5}{|c|}{ p-range } \\
\cline { 2 - 6 } & Ctrl sEV & Doxo sEV & Hs sEV & $\begin{array}{c}\text { Ag Ctrl } \\
\text { SEV }\end{array}$ & $\begin{array}{c}\text { Ag-TiO } \\
\text { sEV }\end{array}$ \\
\hline \multirow{2}{*}{ Cellular development } & $4.45 \mathrm{E}-04-$ & $3.08 \mathrm{E}-04-$ & $1.67 \mathrm{E}-04-$ & $4.09 \mathrm{E}-04-$ & $5.60 \mathrm{E}-04-$ \\
& $5.35 \mathrm{E}-20$ & $3.60 \mathrm{E}-19$ & $2.51 \mathrm{E}-19$ & $8.18 \mathrm{E}-20$ & $3.52 \mathrm{E}-20$ \\
\hline Cellular growth and proliferation & $4.45 \mathrm{E}-04-$ & $3.08 \mathrm{E}-04-$ & $1.67 \mathrm{E}-04-$ & $4.09 \mathrm{E}-04-$ & $5.60 \mathrm{E}-04-$ \\
& $5.35 \mathrm{E}-20$ & $3.60 \mathrm{E}-19$ & $2.51 \mathrm{E}-19$ & $8.18 \mathrm{E}-20$ & $3.52 \mathrm{E}-20$ \\
\hline \multirow{2}{*}{ Cell death and survival } & $4.82 \mathrm{E}-04-$ & $2.47 \mathrm{E}-04-$ & $1.73 \mathrm{E}-04-$ & $3.89 \mathrm{E}-04-$ & $5.92 \mathrm{E}-04-$ \\
& $7.73 \mathrm{E}-20$ & $2.40 \mathrm{E}-21$ & $7.89 \mathrm{E}-25$ & $3.15 \mathrm{E}-19$ & $1.53 \mathrm{E}-18$ \\
\hline \multirow{2}{*}{ Cellular movement } & $4.45 \mathrm{E}-04-$ & $3.08 \mathrm{E}-04-$ & $1.81 \mathrm{E}-04-$ & $4.31 \mathrm{E}-04-$ & $5.88 \mathrm{E}-04-$ \\
& $3.58 \mathrm{E}-15$ & $5.11 \mathrm{E}-14$ & $5.03 \mathrm{E}-12$ & $5.97 \mathrm{E}-14$ & $3.58 \mathrm{E}-14-$ \\
\hline \multirow{2}{*}{ Cell-to-cell signalling and } & $4.81 \mathrm{E}-04-$ & $3.05 \mathrm{E}-04-$ & $1.77 \mathrm{E}-04-$ & $4.02 \mathrm{E}-04-$ & $5.92 \mathrm{E}-04-$ \\
interaction & $2.71 \mathrm{E}-07$ & $1.12 \mathrm{E}-07$ & $2.40 \mathrm{E}-08$ & $3.77 \mathrm{E}-08$ & $3.99 \mathrm{E}-07$ \\
\hline \multirow{2}{*}{ Protein synthesis } & $1.53 \mathrm{E}-04-$ & $3.12 \mathrm{E}-04-$ & $1.96 \mathrm{E}-04-$ & $9.31 \mathrm{E}-05-$ & $9.89 \mathrm{E}-05-$ \\
& $4.69 \mathrm{E}-06$ & $4.21 \mathrm{E}-08$ & $1.55 \mathrm{E}-12$ & $5.51 \mathrm{E}-07$ & $1.00 \mathrm{E}-06$ \\
\hline
\end{tabular}

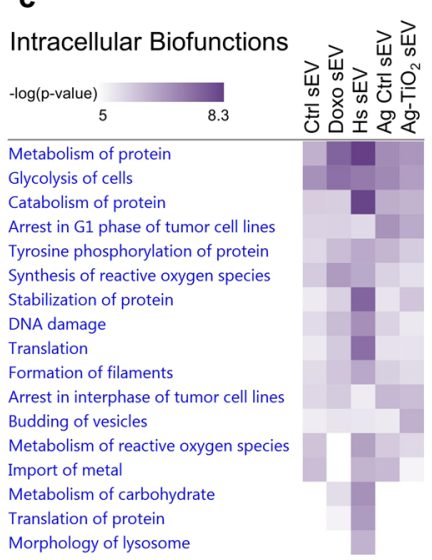

d

Cellular Biofunctions

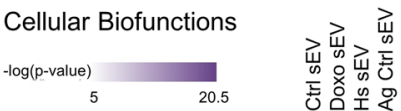

Necrosis

Cell proliferation of tumor cell lines

Cell death

Apoptosis of tumor cell lines

Cell movement of tumor cell lines

Migration of tumor cell lines

Apoptosis

Cell death of cancer cells

Cell death of tumor cells

Cell movement

Cell viability of tumor cell lines

Cell movement of melanoma cell lines

Cell viability

Cell survivi

Migration of cells

Migration of melanoma cell lines

Interaction of tumor cell lines

Apoptosis of tumor cells

Binding of tumor cell lines

Cell movement of endothelial cells

Migration of endothelial cells

Apoptosis of cancer cells

Detachment of cells

Size of cells

Cell spreading of endothelial cells

Colony formation of tumor cell lines

Formation of extracellular matrix

Quantity of cells

Proliferation of tumor cells

Endothelial cell development

Movement of vascular endothelial cells

Cell proliferation of fibroblasts

Binding of endothelial cells

Cell death of connective tissue cells

Cellular homeostasis

\section{e}

Systemic Biofunctions

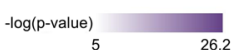

Early stage tumor

Early stage solid tumor

Advanced malignant tumor

Primary melanoma

Secondary tumor

Advanced malignant solid tumo

Metastatic solid tumor

Invasion of cells

Primary solid tumor

Invasion of tumor cell lines

Growth of lesion

Stage I-II cancer

Primary tumor

Metastatic melanoma

Growth of tumor

Fibrosis

Metastasis of cells

Angiogenesis

Stage I cancer

Vasculogenesis

Development of vasculature

Cancer of cells

Angiogenesis of tumor

Fibrogenesis

Cancer

Progression of tumor

Malignant solid tumor

Necrosis of tumor

Growth of malignant tumor

Solid tumor

\section{f}

Immune Biofunctions
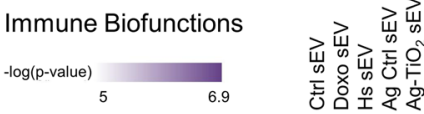

Transmigration of leukocytes

Activation of blood cells

Cell movement of monocytes

Transmigration of cells

Activation of leukocytes

Activation of cells

Detachment of blood cells

Cell rolling of leukocytes

Cellular infiltration

Cell movement of phagocytes

Detachment of granulocytes

Immune response of tumor cell lines

Binding of blood cells

Binding of leukocytes

Adhesion of blood cells

Migration of granulocytes

Adhesion of immune cells

Cell tethering or rolling of leukocytes

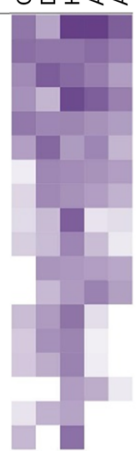

Figure 4. IPA showed that sEVs may influence many biological pathways and functions with different significance via their miRNA and protein content. (a) 'Top 5 canonical pathways' for each sEV group. Red values label the significance of pathways, which were not included in the Top 5. (b) 'Top 5 molecular and cellular functions' for each sEV group. Red values label the significance ranges of functions, which were not included in the Top 5. (c-f) Heatmaps from the 'Comparison analysis' of the molecular content of vesicles. Relevant 'Biofunctions' with $-\log (\mathrm{p}$-value $)>5$ were organised into four groups, namely intracellular, cellular, systemic and immune processes.

'Top 5 molecular and cellular functions' also showed similarities across the sEV groups. 'Cellular development,' 'Cellular growth and proliferation,' 'Cell death and survival' and 'Cellular movement' were shared between each sEV group; 'Protein synthesis' was listed in Doxo and Hs sEVs and 'Cell-to-cell signalling and interaction' 
in $\mathrm{Ctrl}, \mathrm{Ag} \mathrm{Ctrl}$ and $\mathrm{Ag}-\mathrm{TiO}_{2}$ ones (Fig. 4b). All of these cellular functions are involved in tumour progression, which highlights the role and diverse effects of vesicles in recipient cell reprogramming in the tumour matrix and the metastatic sites ${ }^{4-7,56-58}$.

Performing 'Comparison analysis' in the IPA, we built a heatmap of the melanoma-related 'Diseases and Biofunctions', significantly influenced by any sEV group (- $\log (\mathrm{p}$-value $)>5)$. This in silico analysis revealed that the sEVs may play a role not only in intracellular and cellular, but in systemic and immunological processes as well (Fig. 4c-f). Focusing on the activation and inhibitory effects, we identified many 'Biofunctions', which may be regulated differently by the sEVs, highlighting the role of the releasing conditions in the vesicular communication of melanoma cells. In order to prove the tumour-associated functional differences between sEV groups, some 'Biofunctions' related to stem cell proliferation, cell cycle, migration of tumour cells and aggregation of cells were selected for in vitro investigations.

In silico predictions-based in vitro analyses of $\mathrm{sEV}$-induced cellular responses in tumour matrix cells. In regulation-focused examinations using the LC-MS/MS and SOLiD sequencing data, the 'Grow tool' of IPA enabled to identify the interacting vesicular molecules for the investigated 'Biofunctions'. Then, the 'Molecule Activity Predictor' (MAP) feature of IPA predicted their overall regulatory effects for each sEV group. Following these in silico studies, predicted alterations of the $\mathrm{sEV}$-induced cell responses were analysed experimentally by in vitro methods. Proliferation, cell cycle dynamics, migration capacity and microtissue generation of the sEV recipient cells were investigated by Ki-67 immunocytochemistry, Cell-Clock cell cycle assay, wound healing assay and hanging drop technology, respectively.

$\mathrm{Ag}-\mathrm{TiO}_{2} \mathrm{sEVs}$ facilitate proliferation of mesenchymal stem cells. As an important element of the TME, the MSCs can be targeted by the tumour-derived extracellular vesicles. Therefore, we investigated the effects of the different sEVs on stem cells. First, the in silico analyses predicted activation of Ki-67 expression for the Ctrl, Hs and $\mathrm{Ag}-\mathrm{TiO}_{2} \mathrm{sEVs}$, and activation of 'Proliferation of stem cells' for each sEV group. These predictions suggest that after internalisation by stem cells, each of the investigated sEV groups may induce cell divisions and three of them could result in Ki-67 upregulation, if the delivered vesicular cargo is active in the recipient cells (Fig. 5a). According to the IPA analyses, the key $\mathrm{B} 16 \mathrm{~F} 1$ vesicular regulator of the Ki-67 expression may be the aldo-keto reductase family 1 member $\mathrm{B} 1$ (AKR1B1), which is known to be involved in glucose metabolism, osmoregulation, detoxication of lipid aldehydes ${ }^{59}$, oxidative stress signalling, activation of NF- $\kappa B$ and expression of adhesion molecules, such as ICAM or VCAM. It has recently been shown that inhibition of AKR1B1 prevented proliferation and expression of Ki-67 in the human umbilical vein endothelial cells (HUVEC) ${ }^{60}$. The IPA-predicted activation of 'Proliferation of stem cells' might be enhanced for instance by fibronectin (FN1) ${ }^{61}$, which was previously described by Sharma et al. to increase the growth of embryonic stem cells ${ }^{61}$ (Fig. 5a).

To test the differences in the Ki-67 regulation across sEV groups, we treated MSC cultures with $200 \mu \mathrm{g} / \mathrm{ml}$ sEV suspensions or PBS buffer as a negative control. After $24 \mathrm{~h}$ or $72 \mathrm{~h}$ of vesicle exposures, the Ki-67 expression was investigated by immunocytochemistry. For the quantitative evaluation of the experiment, the Operetta high-content imaging system and an image analysis and machine learning software (SCT Analyzer 1.0) ${ }^{62}$ was applied, which enabled to analyse almost $1.6 \times 10^{5}$ cells within a few hours. Our computer-assisted image analysis pipeline was comprised of cell segmentation, feature extraction and machine learning modules, where we had a training set with two classes for the Ki-67 positive and the negative cells. Compared to the negative control group, $\mathrm{Ag}-\mathrm{TiO}_{2}$ sEVs significantly increased the proportion of $\mathrm{Ki}-67$ positive cells after $72 \mathrm{~h}(\mathrm{p}=0.03572, \mathrm{n}=4$; Fig. 5b,c).

Proliferation of MSCs was also tested by direct cell counting, where all of the cells in the sEV-exposed cell cultures were counted using DAPI staining, imaging and machine learning. Results showed increased proliferation of cells upon exposure to $\mathrm{Hs}$ and $\mathrm{Ag}-\mathrm{TiO}_{2}$ sEVs as early as $24 \mathrm{~h}$, but different sEVs each had a distinct influence on this cell function (Fig. 5d).

Our in vitro results suggest that melanoma sEVs released under different microenvironmental conditions may have distinct effect on stem cell proliferation. However, beside the IPA predicted interactions, additional molecules and factors, such as the encapsulated doxorubicin, may also be involved in this process.

Previously, tumour exosomes derived from melanoma cells ${ }^{17}$, osteosarcoma cells ${ }^{63}$ or breast cancer cells ${ }^{64}$ have been shown to re-educate MSCs and provide them tumour-promoting properties. The tumour-educated stem cells may go through an oncogenic reprogramming resulting in increased proliferation capacity in vitro, and tumour growth- and metastasis progression-supporting effects in vivo. Here, we also observed an increase in the proliferation and Ki-67 expression upon the normal (Ctrl) sEV exposure. However, our results suggest that the stem cell re-education capacity of vesicles strongly depends on the microenvironmental conditions of the releasing tumour cells.

Doxo and Ctrl sEVs affect the cell cycle of melanoma cells. IPA analyses predicted inhibition of 'G1 phase of tumour cell lines' and 'G1/S phase transition of tumour cell lines' upon exposure to Ctrl, Doxo and Hs sEVs (Fig. 6a). In other words, the molecular content of these vesicles may cause an arrest in the G1 phase in recipient tumour cells. As displayed on Fig. 6a, IPA found a total of 15 sEV molecules in our B16F1 data, which may influence the G1 phase of tumour cell lines. Hypothetically, the key player of their inhibitory effect may be the aspartyl-tRNA synthetase (DARS). In 1999, Yamashita et al. ${ }^{65}$ investigated T24 bladder carcinoma cells and showed that DARS causes a retinoblastoma-independent downregulation of cyclin A, which is required for $\mathrm{S}$ phase entry ${ }^{65}$. Another component of the B16F1 vesicles, the p53 inducible miR-34a may lead to apoptosis and cell cycle arrest in the G1 phase, thereby suppressing tumour cell proliferation ${ }^{66}$. Ji et al ${ }^{67}$ showed that restoration of miR-34 expression in pancreatic cancer cells inhibited cell growth and invasion, induced apoptosis, arrested cell cycle in G1 and G2/M phases and sensitized the cells to chemotherapy and radiation ${ }^{67}$. 

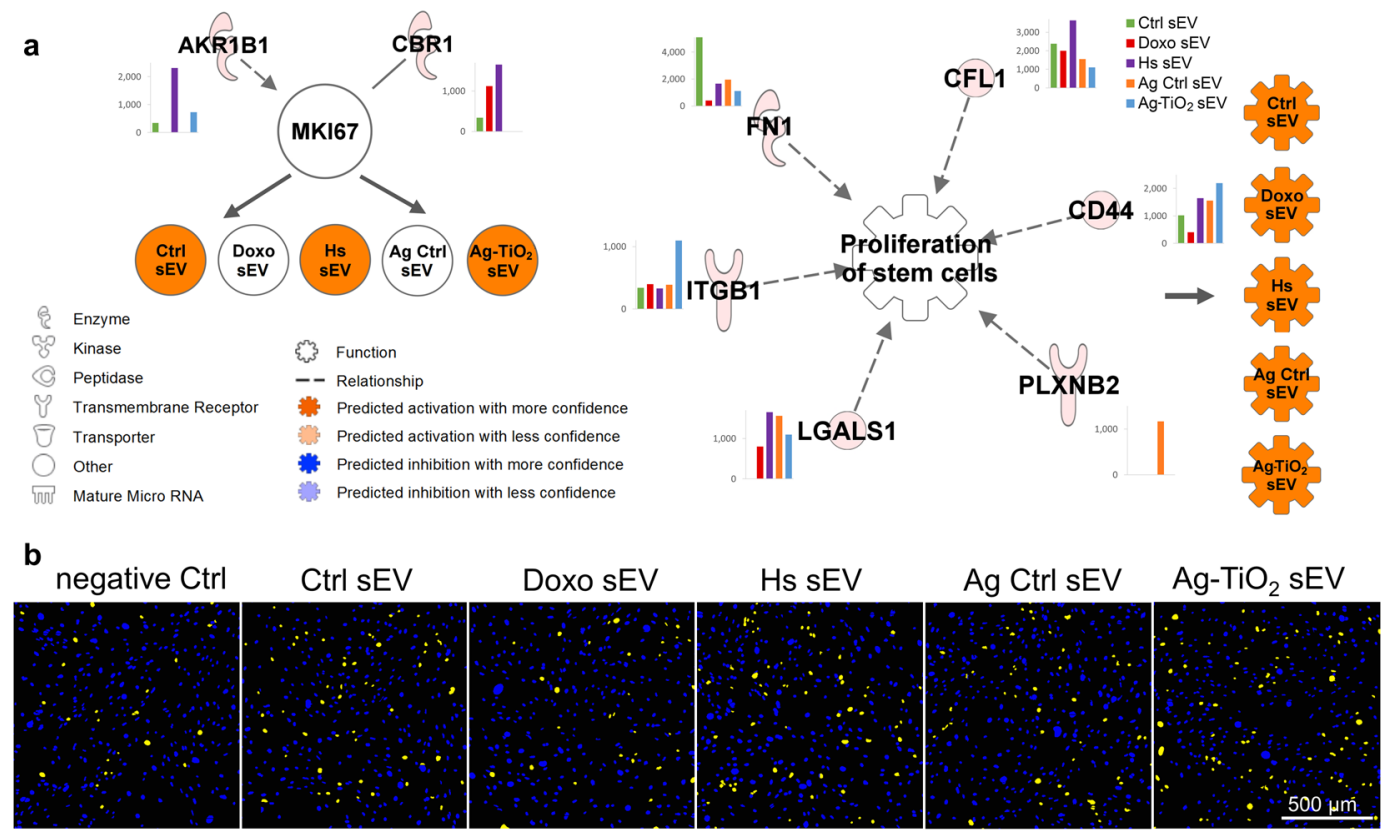

C
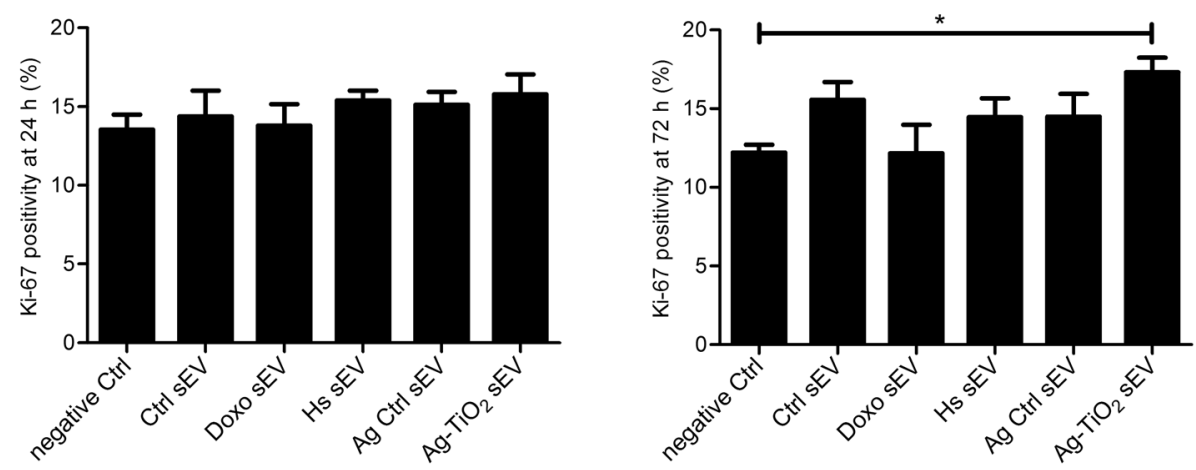

d
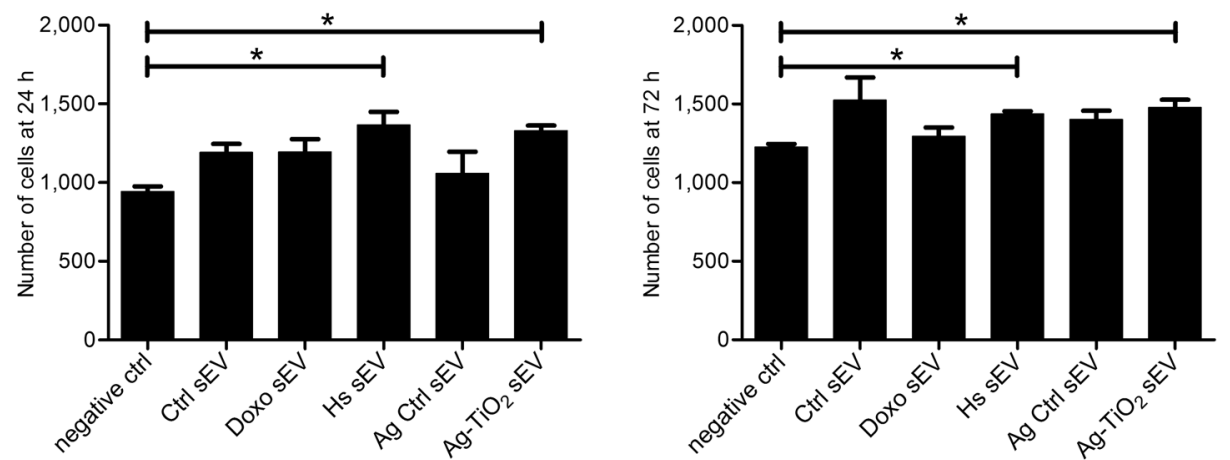

Figure 5. Stress-exposed melanoma cell-derived vesicles affected the proliferation of MSCs. (a) IPA predictions for the regulatory effects of sEV molecules on Ki-67 expression and 'Proliferation of stem cells'. Networks show every upstream regulator proteins accompanied by a bar graph, which represents the normalised expression values of the molecule for each sEV group. Coloured symbols, named as the sEV groups, display the expected regulation changes of the analysed 'Molecule' and 'Biofunction' upon exposure to the vesicles. (b,c) Evaluation of the Ki-67-specific immunocytochemistry using an image analysis and machine learning software. (b) Images are representatives of the classified ones. Yellow and blue dots show the Ki-67 positive and negative nuclei, respectively. (c) Bar graphs show percentages of the Ki-67 positive cells $24 \mathrm{~h}$ (left panel) and $72 \mathrm{~h}$ (right panel) after $\mathrm{sEV}$ exposures. Each bar represents mean $+\mathrm{SD}(\mathrm{n}=4)$. (d) Cell numbers of the sEV-exposed cells after $24 \mathrm{~h}$ and $72 \mathrm{~h}$ incubation time. Bar graphs represent mean $+\mathrm{SD}$ values $(\mathrm{n}=3),{ }^{*} \mathrm{p}<0.05$ indicates statistical significance.

To test the in silico predicted effects of sEVs on tumour cell cycle, we performed a Cell-Clock cell cycle assay on B16F1 melanoma cells exposed to sEVs for $24 \mathrm{~h}, 48 \mathrm{~h}$ and $72 \mathrm{~h}$ along with a PBS-treated negative control group. This assay utilizes a vital redox dye that changes colour based on the cell cycle phase. It becomes yellow in G1, 

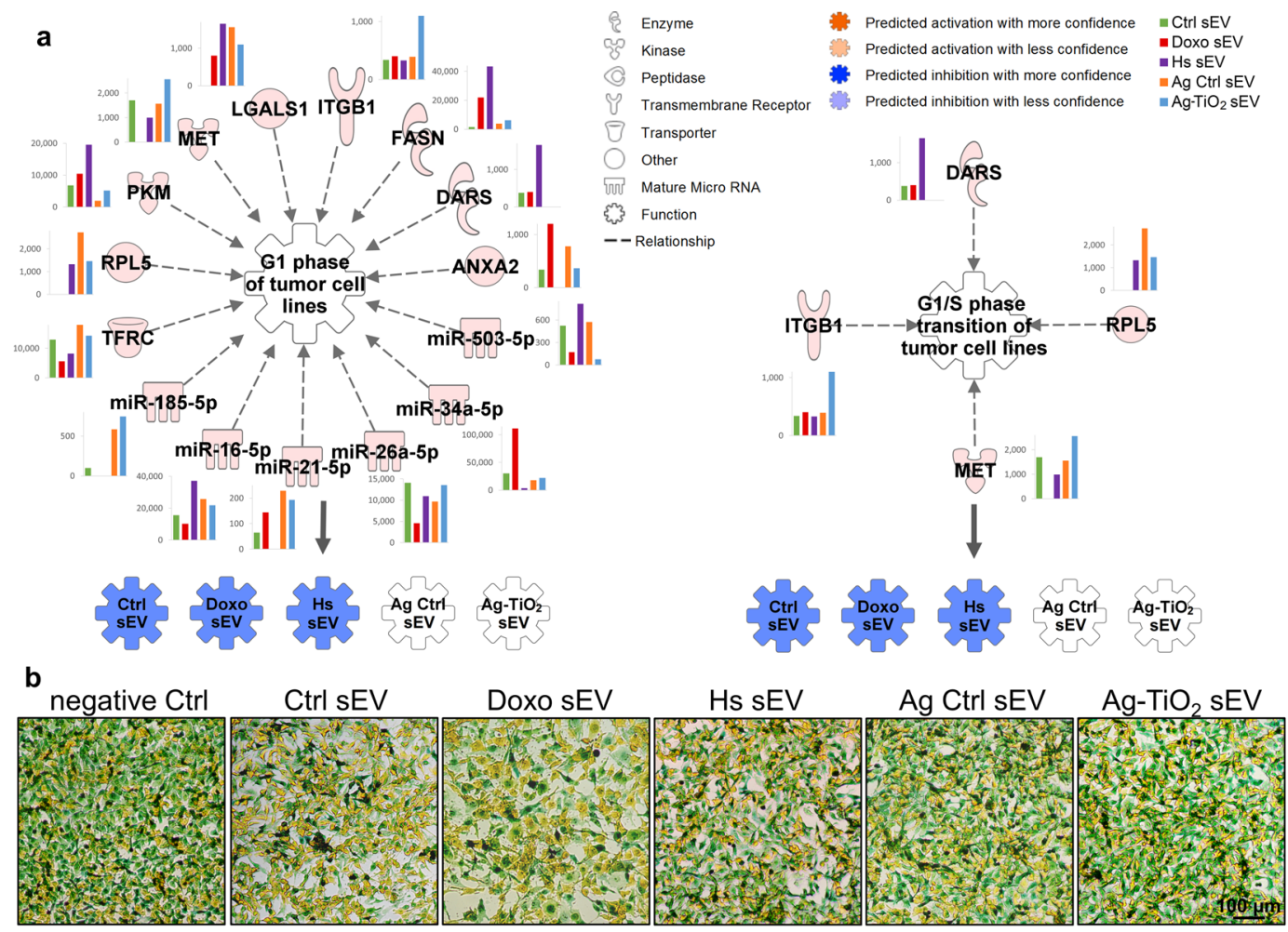

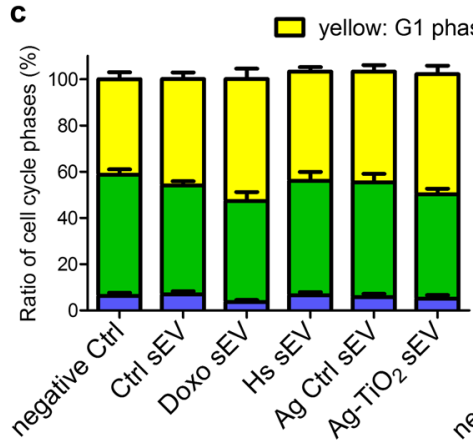

$24 \mathrm{~h}$

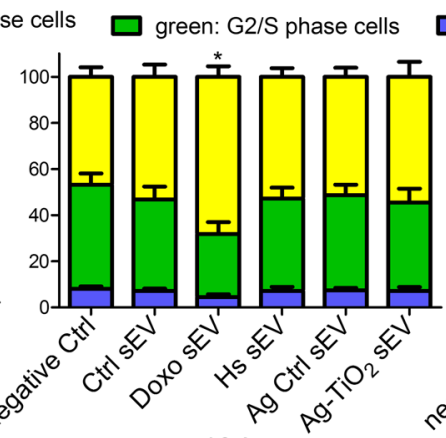

$48 \mathrm{~h}$ blue: M phase cells

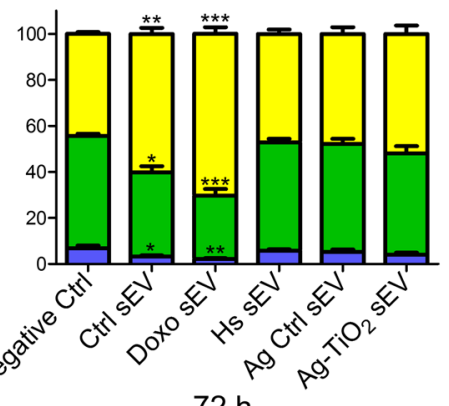

Figure 6. Ctrl and Doxo sEVs caused an arrest in G1 phase of melanoma cells. (a) IPA predictions for the regulatory effects of sEV molecules on the 'G1 phase of tumour cell lines' and 'G1/S phase transition of tumour cell lines'. Networks show every upstream regulator proteins and miRNAs accompanied by a bar graph, which represents the normalised expression values of the molecule for each sEV group. Coloured symbols, named as the sEV groups, display the expected regulation changes of the analysed 'Biofunctions' upon exposure to the vesicles. (b,c) Cell-Clock cell cycle assay of sEV-exposed B16F1 cell cultures. (b) Representative images of the cell clock dye-labelled cultures. (c) Distribution of the yellow, green and blue cells in the cell cultures, which labels the G1, G2/S and M phase cells, respectively. Each bar represents mean $+\mathrm{SD}(\mathrm{n}=4),{ }^{*} \mathrm{p}<0.05$, $* * \mathrm{p}<0.01$ and $* * * \mathrm{p}<0.001$ indicate statistical significance.

green in S/G2 and blue in M phase. In the negative Ctrl group, cells were present in the G1, S/G2, and M phases in average proportions of $44.84 \pm 2.68 \%, 47.80 \pm 3.34 \%$ and $7.35 \pm 0.81 \%$, respectively $(n=12)$. Ctrl and Doxo sEVs led to an increase in the proportion of the yellow, i.e. G1 phase, cells in a time dependent manner. After $72 \mathrm{~h}$, these cells represented $59.20 \pm 4.06 \%$ of the Ctrl sEV-exposed cultures $(\mathrm{p}=0.00346, \mathrm{n}=4)$ and $70.32 \pm 7.24 \%$ of Doxo sEV-exposed cultures $\left(\mathrm{p}=4.28 \times 10^{-6}, \mathrm{n}=4\right)($ Fig. $6 \mathrm{~b}, \mathrm{c})$. These results confirmed the IPA predicted arrest in G1 phase by the Ctrl and Doxo, but not by the Hs sEVs.

It has been previously demonstrated that tumour cells can efflux drugs through exosome secretion ${ }^{43,68}$. Yang et al. showed that doxorubicin-treated MCF-7 breast carcinoma cells produced drug-containing exosomes. Doxorubicin that was encapsulated in the MCF-7 exosomes had more potent cytotoxicity against the parental MCF-7 cells than the free drug ${ }^{43}$. Furthermore, doxorubicin arrest the cell cycle of tumour cells at G1/S and G2/M checkpoints ${ }^{69,70}$. Based on these literature data and our fluorescence spectroscopy measurements, we hypothesize that encapsulated doxorubicin might contribute to the enhanced cell cycle arrest effect of Doxo sEVs. 
a
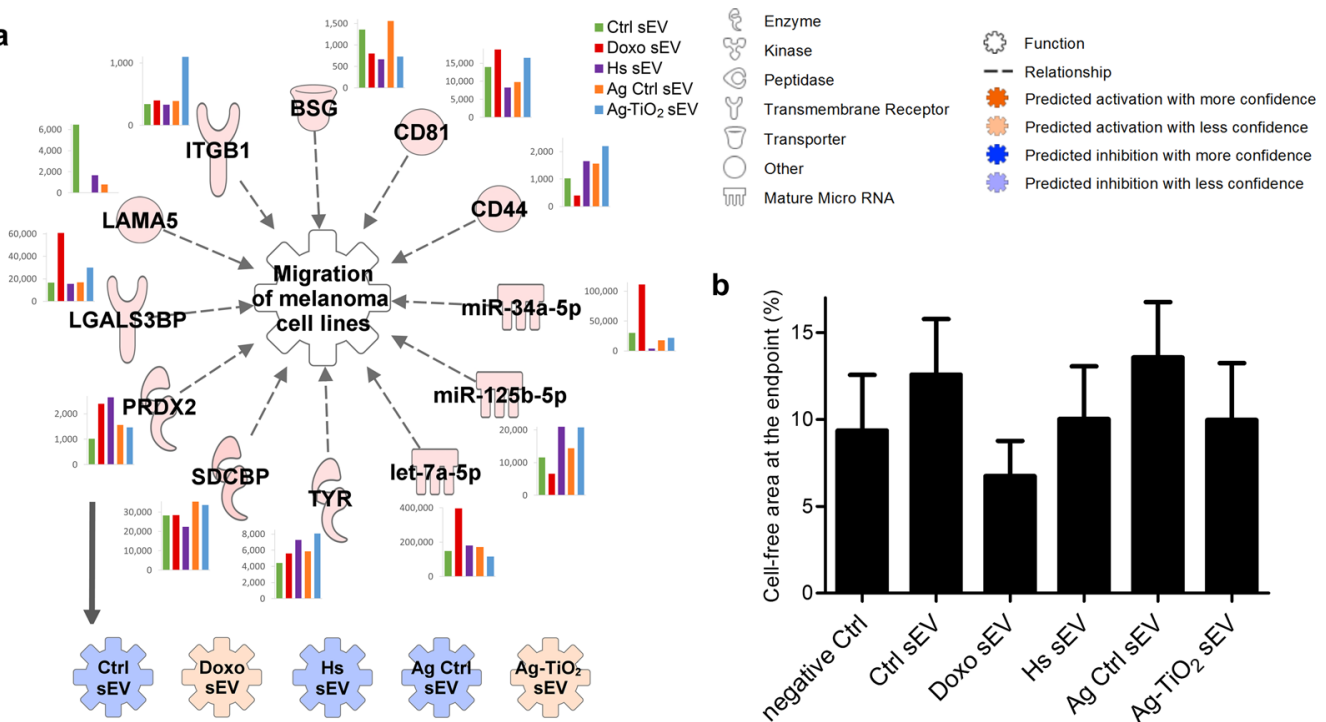

C

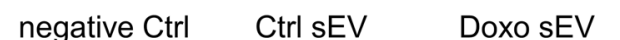

b
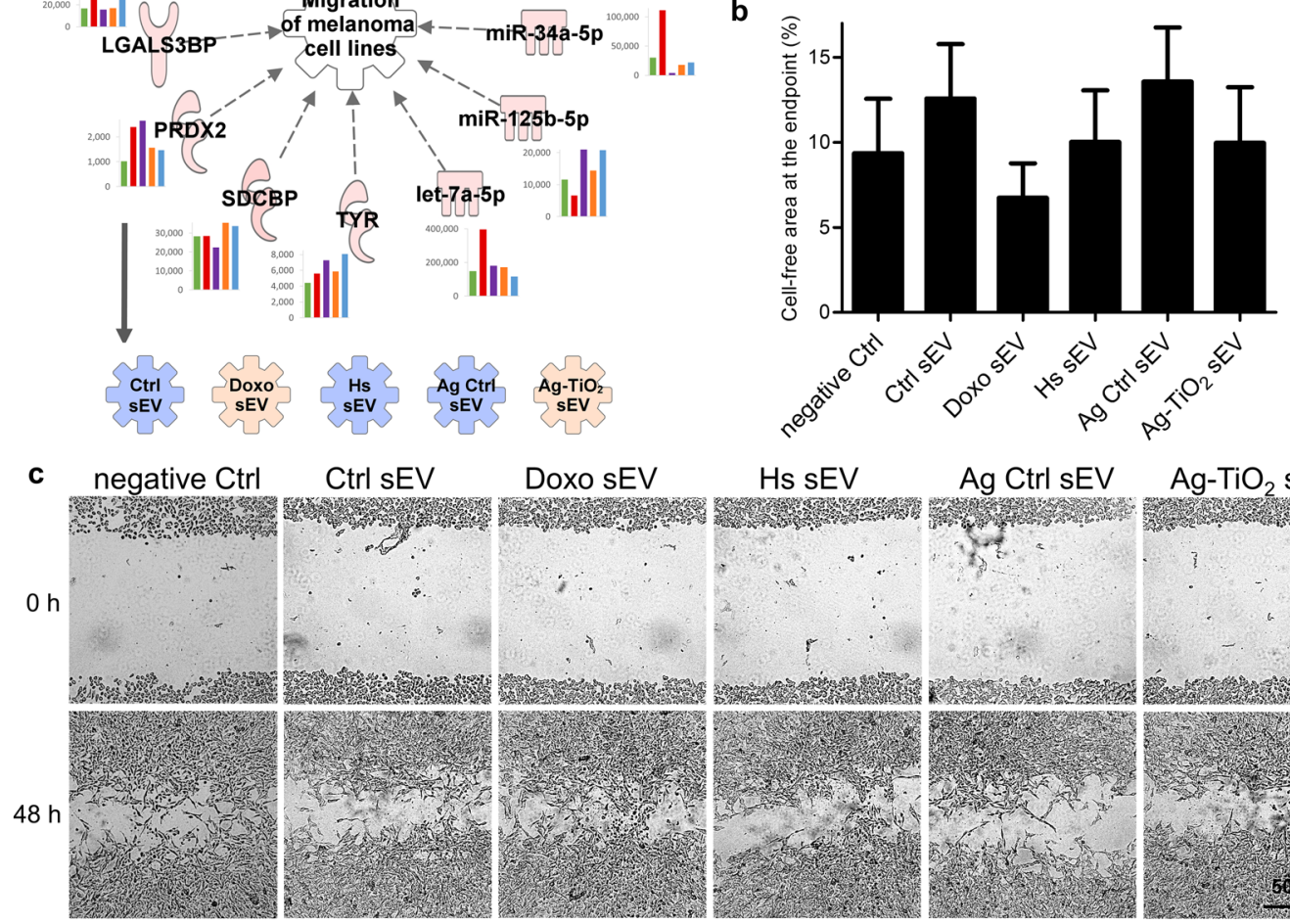

Ag Ctrl sEV

$\mathrm{Ag}-\mathrm{TiO}_{2} \mathrm{sEV}$

Figure 7. Doxo sEVs enhanced the migration of melanoma cells. (a) IPA predictions for the regulatory effects of sEV molecules on the 'Migration of melanoma cell lines'. Network shows every upstream regulator proteins and miRNAs accompanied by a bar graph, which represents the normalised expression values of the molecule for each sEV group. Coloured symbols, named as the sEV groups, display the expected regulation changes of the analysed 'Biofunction' upon exposure to the vesicles. (b,c) Wound healing assay of sEV-exposed B16F1 cell cultures. (b) The bar graph shows the result of the analysis of wound closures by the ImageJ wound healing tool. It represents mean $+\mathrm{SD}$ values $(\mathrm{n}=8)$. (c) Representative images of the wounds after $48 \mathrm{~h}$ of sEV exposures.

Migration capacity of melanoma cells is differently altered by the sEVs. From many cell movement-related 'Biofunctions', which were predicted to be targeted by sEVs (Fig. 4d-f), we chose the 'Migration of melanoma cell lines' for further IPA and in vitro investigations. The in silico analyses showed varying sEV effects. More specifically, Doxo and $\mathrm{Ag}-\mathrm{TiO}_{2} \mathrm{sEVs}$ are predicted to facilitate the melanoma cell migration, while the three other ones may inhibit this function (Fig. 7a). According to the IPA analyses, negative B16F1 sEV regulators may include the laminin subunit alpha-5 (LAMA5), peroxiredoxin-2 (PRDX2), tyrosinase (TYR), let-7a-5p, miR-125b-5p and the miR-34a-5p. Listed positive regulators include the CD44 antigen (CD44), CD81 antigen (CD81), basigin (BSG), integrin beta-1 (ITGB1) and the galectin-3-binding protein (LGALS3BP) (Fig. 7a). Using attachment and pulmonary metastases assays, Hibino et al. ${ }^{71}$ identified four peptides of LAMA5, which showed activity in vitro and also in vivo. These peptides reduced migration and invasion of B16F10 melanoma cells ${ }^{71}$. Lee et al. ${ }^{72}$ showed that the PRDX2 enzyme is a selective antioxidant suppressor for proliferation and migration of melanoma cell lines (SK-MEL-5, SK-MEL-28, A375, G361, B16F10) ${ }^{72}$. Stampolidis et al. demonstrated that LGALS3BP promotes cell viability and facilitates cell motility of the human C8161 melanoma cell line $\mathrm{e}^{73}$.

Using wound healing assay, we investigated the effect of sEVs on migration of B16F1 cells, which approximated to the IPA predicted tendency. Migration of cells into the wounded area was slightly decreased in the presence of Ctrl and Ag Ctrl sEVs, compared to migration of the negative Ctrl cells. Acceleration of wound closure was observed in response to Doxo sEVs $(n=8)$. However, Hs and $\mathrm{Ag}-\mathrm{TiO}_{2}$ sEVs had no effect on tumour cell migration (Fig. $7 \mathrm{~b}, \mathrm{c}$ ).

The importance of migration capacity in tumour progression is unquestionable, since the process of tumour cell invasion and metastasis is conventionally understood as the migration of individual cells, which detach from the primary tumour, enter lymphatic vessels or the bloodstream and seed in distant organs ${ }^{74}$. This cancer cell migration is typically regulated by integrins, matrix-degrading enzymes, cell-cell adhesion molecules and cell-cell communication $^{74}$. Direct and indirect effects of stress-elicited sEVs on tumour cell migration and metastasis 
have been demonstrated in some studies ${ }^{27,47,75}$. Here, we amended literature data by showing that the cytostatic stress-exposed cell-derived sEVs enhance the migration of the recipient melanoma cells. This can be interpreted as an adaptive escape mechanism: Doxo sEVs, delivering a warning message, induce the migration of the neighbouring melanoma cells. In a recent study ${ }^{27}$, breast cancer cells also showed an sEV-mediated escape mechanism under doxorubicin and paclitaxel exposures, by releasing pro-metastatic exosomes ${ }^{27}$.

Each sEV group enhances the migration of endothelial cells. IPA predicted the activation of 'Cell migration of endothelial cells' and 'Cell spreading of endothelial cells' upon exposure to each sEV group labelling a large number of potentially contributing sEV molecules (Supplementary Fig. S5). Therefore, we repeated the wound healing assays on bEnd. 3 mouse endothelial cells, where we could verify the IPA predictions. However, Ctrl sEVs showed the highest migration enhancing effect (Supplementary Fig. S5).

Endothelial cell migration is an essential component of angiogenesis, which is a key process of tumour progression $^{76}$. These results demonstrate that some type of stress conditions may slightly decrease the endothelial cell migration promoting effects of sEVs. At the same time, they highlight that sEVs may have target cell-specific functional effects in the recipients, which further increases the diversity of the sEV-mediated communication of melanoma cells.

Microtissue generation is facilitated independently of the sEV groups. IPA predicted that each of the five sEV groups may activate many 'Biofunctions' related to the formation of a 3D cell interaction matrix, e.g. 'Aggregation of cells', 'Formation of ECM' (Fig. 8a), 'Cell-cell contact' or 'Interaction of tumour cell lines' (Supplementary Fig. S6). The intensity of these activations is variable between sEV groups, for example activation of the 'Aggregation of cells' is predicted to be the strongest upon Doxo and Hs sEV exposures. In silico analyses revealed a large number of contributing B16F1 sEV molecules. For instance, it is worth to mention the programmed cell death 6-interacting protein (PDCD6IP, Alix). It has an important role not only in the exosome biogenesis ${ }^{52}$, but Pan et al. ${ }^{77}$, who investigated fibroblast morphology, demonstrated that a sub-population of Alix localises extracellularly and regulates integrin-mediated cell adhesions and fibronectin matrix assembly ${ }^{77}$.

In order to mimic in vivo conditions, we established a simplified 3D tumour matrix model co-culturing MSCs and B16F1 cells in hanging drop plates. By definition, multicellular cell aggregates, which produce their own ECM and are comprised of more than one cell types are termed as microtissues ${ }^{78}$. Applying an equal number of the two cell types (a total of 5,000 cells/well), microtissue generation was followed under sEV exposures and PBS treatment as a negative control for $72 \mathrm{~h}$. The created microtissues were photographed and analysed by the AnaSP software developed for automatic image analysis of multicellular spheroids ${ }^{79}$. Each group of sEVs resulted in smaller and more compact microtissues, since the average values of the measured area, perimeter, diameter and volume was lower, compared to the negative Ctrl samples (Fig. 8b-d). These results were validated using mouse embryonic fibroblasts (MEFs) co-cultured with B16F1 cells, where each sEV group facilitated the microtissue generation, except the Hs sEVs (Supplementary Fig. S7).

In videomicroscopy studies, Crawford et al ${ }^{80}$ observed that microvesicles attaching to the cell membranes promoted cell-to-cell interactions and spheroid formation of glioblastoma, breast carcinoma and osteosarcoma cells. They also showed that elevated extracellular $\mathrm{Ca}^{2+}$ levels promote microvesicle production and result in smaller and less dense spheroids, which might seem paradoxical. However, they interpreted their results as a pathophysiological adaptation, since increasing spheroid surface to volume ratio, thereby increasing the surface area enhances the uptake of oxygen, growth factors and nutrients by tumour cells that make up the spheroid mass $^{80}$. In our experimental setup, sEV exposures resulted in smaller microtissues from the same number of cells suggesting the generation of more compact structures, which may be explained by the role of vesicles in generation of cell-cell associations, and may also indicate an adaptation mechanism.

Tumours exist in a 3D microenvironment, in which morphological and functional properties, such as the ECM, cell-cell interactions, oxygen gradient and acid gradient create transport barriers for drug delivery ${ }^{81}$. In this study, Doxo sEV exposure resulted in the most compact structures, which may reduce penetration of drugs. Previously, tumour-derived extracellular vesicles have been shown to transfer drug resistance into other tumour cells $^{82,83}$ suggesting that the Doxo sEVs might convey protective messages resulting in more compact microtissues.

\section{Conclusions}

In this study, we compared different inducible cellular stress conditions, and we found that cytostatic, heat and oxidative stresses resulted in changes in the vesicular cargo composition, leading to distinct functional alterations of the melanoma-derived sEVs in the TME recipient cells.

We showed that sEVs, being complex information packages may participate in a wide range of signalling pathways. The fact that a vesicular molecular pattern with a large number of molecules can influence the activation of the cellular homeostasis network at several points, suggests a huge diversity of sEV functions, which sometimes seem to be paradoxical in the in vitro experiments. In conclusion, hundreds of vesicular molecules may have thousands of functional effects in the recipient cells leading to an unconceivable outcome. Here, we successfully predicted the functional effects of the investigated sEV molecular patterns - induced by five treatment conditions - by bioinformatics analyses using unique combinations of the IPA approaches. Based on our knowledge, our experimental setup was suitable to model the transfer and functional activity of the vesicular cargo in the recipient cells. Here, we demonstrated, that pathway analyses may provide a good approximation to the prediction of different inducible stress-elicited responses, suggesting that in silico analyses may be useful tools not only in the field of research, but in a therapeutic approach as well.

Our paper not only provides a detailed characterisation of the doxorubicin-, heat- and $\mathrm{Ag}-\mathrm{TiO}_{2}$-induced molecular patterns of melanoma-derived sEVs and the resulted response patterns in the recipient cells, it also increases our knowledge about the molecular and functional complexity as well as condition-dependent variability 
a

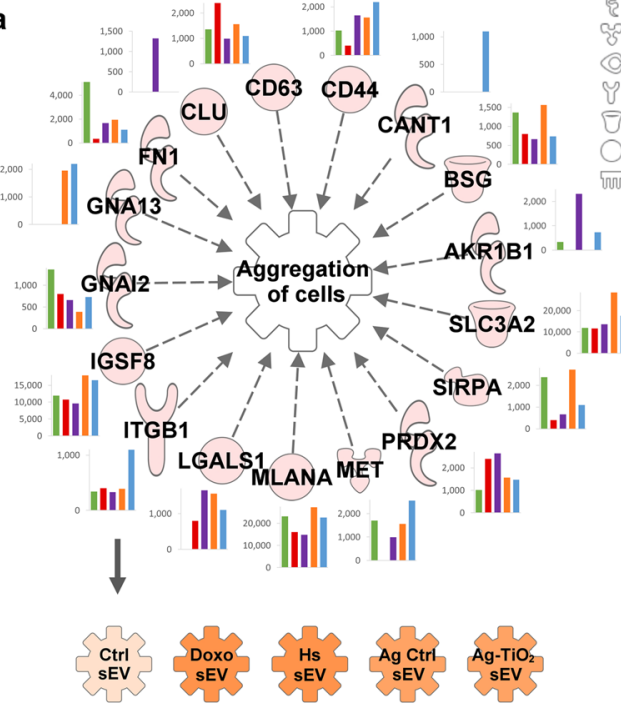

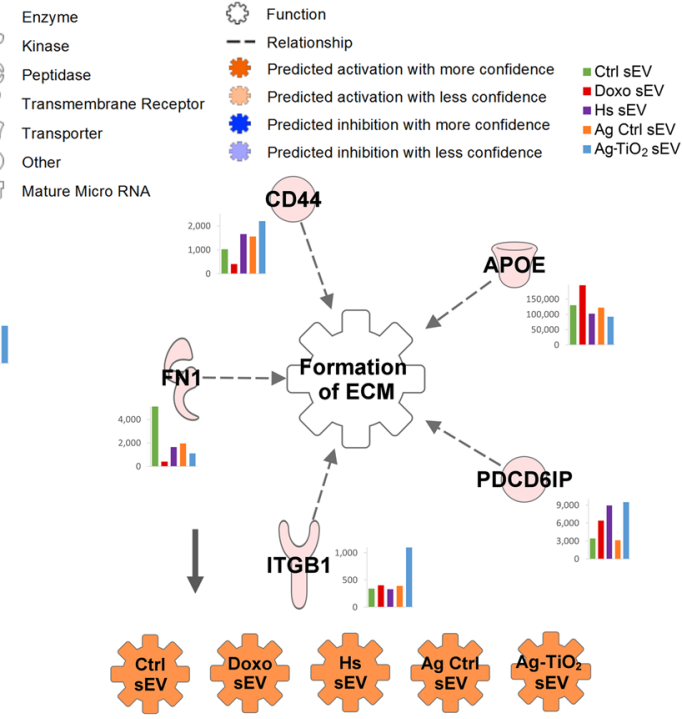

b

\begin{tabular}{|l|c|c|c|c|c|c|}
\hline Group name & negative Ctrl & Ctrl sEV & Doxo sEV & Hs sEV & Ag Ctrl sEV & Ag-TiO $_{2}$ sEV \\
\hline Eq. diameter $(\mu \mathrm{m})$ & $445 \pm 3$ & $315 \pm 7$ & $244 \pm 3$ & $351 \pm 11$ & $371 \pm 5$ & $338 \pm 7$ \\
\hline Major diameter $(\mu \mathrm{m})$ & $521 \pm 21$ & $370 \pm 7$ & $275 \pm 15$ & $430 \pm 31$ & $440 \pm 7$ & $393 \pm 3$ \\
\hline Minor diameter $(\mu \mathrm{m})$ & $307 \pm 56$ & $221 \pm 16$ & $204 \pm 14$ & $242 \pm 61$ & $287 \pm 20$ & $254 \pm 8$ \\
\hline Convexity & $0.801 \pm 0.033$ & $0.799 \pm 0.015$ & $0.911 \pm 0.024$ & $0.808 \pm 0.035$ & $0.779 \pm 0.013$ & $0.759 \pm 0.053$ \\
\hline Solidity & $0.885 \pm 0.022$ & $0.849 \pm 0.014$ & $0.942 \pm 0.019$ & $0.867 \pm 0.03$ & $0.859 \pm 0.019$ & $0.839 \pm 0.061$ \\
\hline Sphericity & $0.731 \pm 0.044$ & $0.701 \pm 0.011$ & $0.865 \pm 0.038$ & $0.727 \pm 0.052$ & $0.694 \pm 0.020$ & $0.667 \pm 0.061$ \\
\hline
\end{tabular}
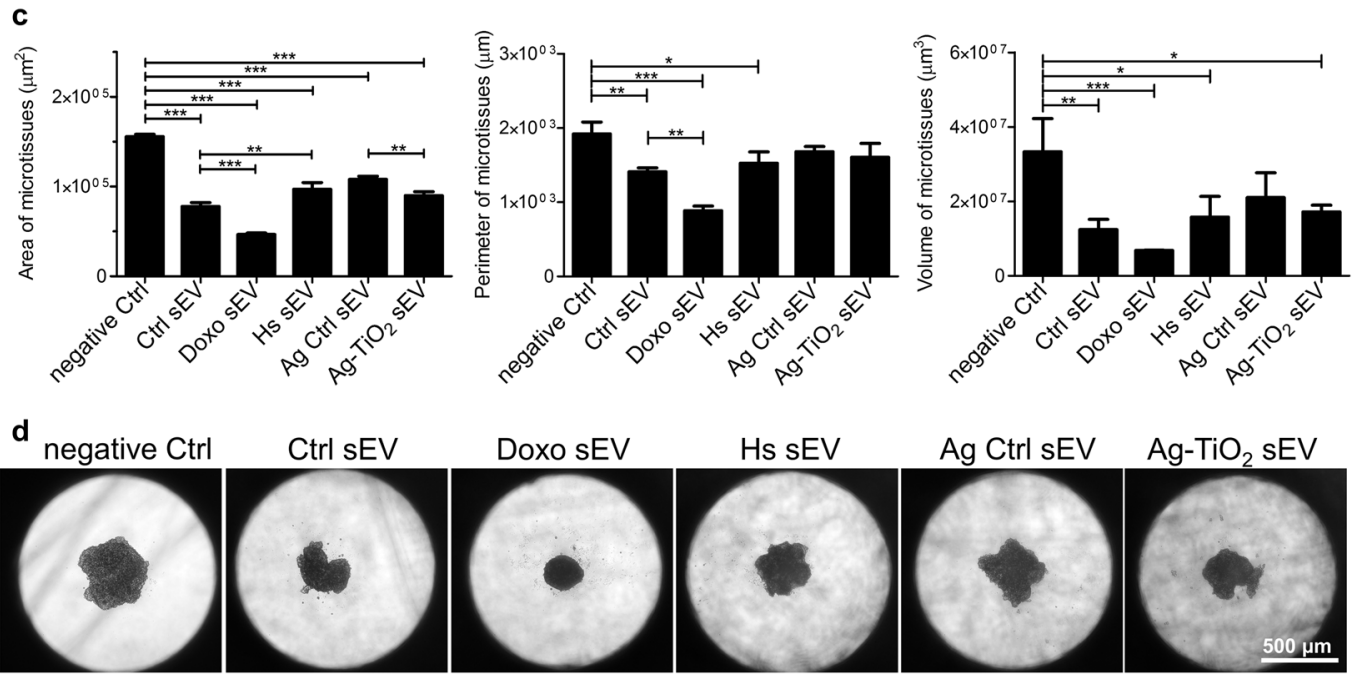

Figure 8. All sEV groups facilitated microtissue generation. (a) IPA predictions for the regulatory effects of sEV molecules on the 'Aggregation of cells' and the 'Formation of extracellular matrix'. Networks show every upstream regulator proteins and miRNAs accompanied by a bar graph, which represents the normalised expression values of the molecule for each sEV group. Coloured symbols, named as the sEV groups, display the expected regulation changes of the analysed 'Biofunctions' upon exposure to the vesicles. (b,c) Descriptive statistics of the $72 \mathrm{~h} \mathrm{B16F1-MSC}$ microtissues resulted from image analysis using the AnaSP software. Eq. diameter means equivalent diameter, major and minor diameters are measured through centroid. Table contains mean \pm SD values. Bar graphs show the area, perimeter and volume statistics of the generated microtissues $($ mean $+\mathrm{SD}, \mathrm{n}=3)$. Statistical evaluation was performed by Welch's ANOVA test with Tukey's HSD post-hoc test; $* \mathrm{p}<0.05, * * \mathrm{p}<0.01$ and $* * * \mathrm{p}<0.001$ indicate statistical significance. (d) Representative images of the generated microtissues after $72 \mathrm{~h}$ of sEV exposures. 
of the melanoma-derived sEVs. This study contributes to a better understanding of the pathogenesis and therapeutic responses of melanoma. It also highlights that indirect effects of any therapy, such as a chemotherapy, may have a great influence on the intercellular communication of the affected cells.

Based on this study, we conclude that the molecular pattern of these highly protected information packages is dictated by the microenvironmental conditions, including the therapeutic stress factors. The altered cargo of sEVs is able to not only enhance or suppress existing signalisation pathways, but even trigger de novo pathway activations, resulting a unique target cell-specific response pattern in the sEV recipient cells. Recent literature data $^{24,25,27,47}$ along with this study suggest that alteration of this complex sEV-mediated intercellular communication of tumour cells deserves special attention among the therapy-induced host responses, which may have a potential influence on the treatment efficacy.

\section{Methods}

Cell cultures. B16F1 (ECACC 92101203) mouse melanoma cell line was obtained from ECACC and cultured in DMEM supplemented by 10\% FBS (EuroClone), $2 \mathrm{mM} \mathrm{L-glutamine} \mathrm{and} \mathrm{1 \%} \mathrm{Penicillin-Streptomycin-}$ Amphotericin B mixture (P/S/A; all from Lonza); mouse embryonic fibroblasts (MEFs; ATCC SCRC-1040) were obtained from ATCC and cultured in DMEM supplemented by 15\% FBS and 1\% P/S/A; bEnd.3 mouse endothelial cell line (ATCC CRL-2299) was obtained from ATCC and cultured in DMEM supplemented by $10 \%$ FBS and $1 \% \mathrm{P} / \mathrm{S} / \mathrm{A}$. Primary mouse mesenchymal stem cells (MSCs) were isolated from adipose tissue of 6-8 week old male C57BL/6N mice (Charles River Laboratories) and cultured using the MesenCult Expansion Kit (STEMCELL Technologies). All cell cultures were maintained in a humidified incubator at $37^{\circ} \mathrm{C}$ and $5 \% \mathrm{CO}_{2}$. Purity of MSC cultures was checked by flow cytometry using the Mouse Multipotent Mesenchymal Stromal Cell Marker Antibody Panel (R\&D Systems) according to the manufacturer's instructions and a FACSCalibur instrument coupled with CellQuest Pro 6.0 (BD Biosciences). Animal experiments were performed in accordance with the national and European animal ethics guidelines. The animal experimental protocols were approved by the Animal Experimentation and Ethics Committee of the Biological Research Centre of the Hungarian Academy of Sciences and the Hungarian National Animal Experimentation and Ethics Board (clearance number: XVI./78/2018).

Ag- $-\mathrm{TiO}_{2}$ photocatalyst particles. Synthesis of $\mathrm{Ag}-\mathrm{TiO}_{2}$ nanoparticles was performed as published earlier ${ }^{31,35}$. Briefly, the silver particles were strongly attached on the surface of metal oxide by photodeposition. A commercially available $\mathrm{TiO}_{2}$ (Degussa P25, Evonik Gmbh) with a specific surface area of $\sim 50 \mathrm{~m}^{2} / \mathrm{g}$ was used for this purpose. The prepared $\mathrm{Ag}-\mathrm{TiO}_{2}$ photocatalyst contained $0.5 \mathrm{wt} \%$ surface silver nanoparticles. Dispersion of particles was made in PBS at a concentration of $10 \mathrm{mg} / \mathrm{ml}$ and sonicated for $30 \mathrm{~min}$ directly before use.

Stress conditions. B16F1 cell cultures were treated at 70\% confluency in five different ways in EV-depleted FBS-containing media for $72 \mathrm{~h}$ (Table 1 ). The Control 1 group received only fresh medium. Cytostatic stressed cells were treated with $0.6 \mu \mathrm{M}$ doxorubicin, heat stressed cells were incubated at $42^{\circ} \mathrm{C}$ for $2 \mathrm{~h}$ in every $24 \mathrm{~h}$ (a total

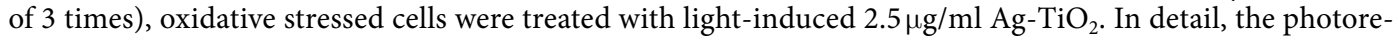
active $\mathrm{Ag}-\mathrm{TiO}_{2}$ nanoparticles were induced by a low-pressure mercury lamp ( $\lambda \geq 360 \mathrm{~nm}, \mathrm{GCL} 303 \mathrm{~T} 5 / 4$ type, LightTech) for $60 \mathrm{~min}$ from $3 \mathrm{~cm}$ distance in a $75 \%$ medium volume to avoid the light absorption and reflection of the medium layer. After the illumination, the medium was adjusted to the final volume. To eliminate the effect of illumination itself, we established another control group, which received medium illuminated in the same way as described above. Parameters of the cytostatic and oxidative stresses were based on previous optimization by proliferation assay. Heat stress conditions were adapted from literature data ${ }^{34}$. In each group, $72 \mathrm{~h}$ supernatants of 6 parallel cell cultures were harvested, pooled and subjected to sEV isolation.

Scanning electron microscopy (SEM). B16F1 cells seeded to poly-L-lysine-coated $5 \mathrm{~mm}$ cover glasses were treated as described above. After $24 \mathrm{~h}$ incubation, cells were washed with PBS and fixed for overnight in $2.5 \%$ glutaraldehyde and $0.05 \mathrm{M}$ cacodylate buffer diluted in PBS ( $\mathrm{pH}$ 7.2). Then, cells were washed with PBS, dehydrated with a graded ethanol series $(30 \%, 50 \%, 70 \%, 80 \% \mathrm{EtOH}$, each for $1 \mathrm{~h}$ and $100 \% \mathrm{EtOH}$, for $3 \times 1 \mathrm{~h}$ ) and dried with a critical point dryer (Quorum Technologies Ltd, K850). Cover glasses were mounted onto microscope stubs using carbon tape, followed by $15 \mathrm{~nm}$ gold coating (Quorum Technologies Ltd, Q150) and observed under a field-emission scanning electron microscope (JEOL Ltd, JSM-7100F/LV). Images were taken in 1,500 $\times$ and 20,000 $\times$ magnification.

sEV isolation and characterisation. Vesicles were isolated by differential filtration and ultracentrifugation. Briefly, $72 \mathrm{~h}$ supernatants from the stress-exposed B16F1 cultures were centrifuged at $780 \mathrm{~g}$ for $5 \mathrm{~min}$, and at $3,900 \mathrm{~g}$ for $15 \mathrm{~min}$ at $4^{\circ} \mathrm{C}$, then filtered by a $0.2 \mu \mathrm{m}$ membrane to remove cells, debris and larger vesicles. Small EVs were pelleted by ultracentrifugation at $150,000 \mathrm{~g}$ for $60 \mathrm{~min}$ at $4^{\circ} \mathrm{C}$ using a T-1270 fixed-angle rotor and a $\mathrm{WX}+$ ultracentrifuge (Sorvall). The pellet was washed twice and resuspended in PBS. Protein concentrations of sEV isolates were measured by the Pierce BCA Protein assay kit (Thermo Scientific) on a benchtop microplate reader (Multiskan RC, Thermo Labsystems) coupled with the Ascent Software 2.6. Small EVs were characterised by atomic force microscopy as described previously ${ }^{44}$, dynamic light scattering using a Zetasizer Nano $\mathrm{S}$ instrument (Malvern Panalytical Ltd) and Western blot analysis (described in the Supplementary Methods). Quantification of sEVs was performed by nanoparticle tracking analysis using a NanoSight NS500 instrument (Malvern Panalytical Ltd).

Fluorescence spectroscopy. The emission and excitation spectra of doxorubicin were measured by a FluoroLog-3 spectrofluorometer (Horiba Ltd). The maximum wavelengths for excitation $\left(\lambda_{\mathrm{ex}}=492 \mathrm{~nm}\right)$ and emission $\left(\lambda_{\mathrm{em}}=592 \mathrm{~nm}\right.$ ) were then used for measuring the fluorescence intensity of Doxo sEVs and Ctrl sEVs as a background. A calibration curve of doxorubicin covering a concentration range of $0-1,000 \mathrm{nM}$ was applied to determine the encapsulated doxorubicin concentration of the sEV samples. 
Dynamic light scattering (DLS) measurements of $\mathrm{Ag}-\mathrm{TiO}_{2}$ particles. The particle size values of the $\mathrm{Ag}-\mathrm{TiO}_{2}$ photocatalyst particles were determined DLS with a Zetasizer Nano ZS ZEN 4003 apparatus (Malvern Panalytical Ltd) equipped with a He-Ne laser $(\lambda=633 \mathrm{~nm})$. The measurements were performed in B16F1 culture medium for a $72 \mathrm{~h}$ time interval. Size distribution measurements were carried out in triplicate, and mean $\pm S D$ values are reported.

Chemiluminescence detection of $\mathrm{Ag}-\mathrm{TiO}_{2}$ nanoparticles. Isolated $\mathrm{Ag} \mathrm{Ctrl} \mathrm{and} \mathrm{Ag}-\mathrm{TiO}_{2} \mathrm{sEVs}$ were lysed by TENT buffer and freeze-thaw cycle, then a 10 -fold, 5 -step Ag- $\mathrm{TiO}_{2}$ nanoparticle dilution series $(0-2.5 \mu \mathrm{g} /$ $\mathrm{ml}$ ) was prepared in $\mathrm{Ag}$ Ctrl sEV suspension for calibration. Protein concentration of sEV lysates was $200 \mu \mathrm{g} / \mathrm{ml}$. After 30 min illumination on 96-well plates, $50 \mu \mathrm{l} 3.38 \mathrm{mM}$ luminol solution was added to $50 \mu \mathrm{l}$ of samples and light emission was immediately detected by a Luminoskan Ascent Microplate Luminometer (Thermo Scientific). Each sample was measured in triplicates.

Transmission electron microscopy (TEM). The morphology of $\mathrm{Ag}-\mathrm{TiO}_{2} \mathrm{sEVs}$ and $\mathrm{Ag}-\mathrm{TiO}_{2} \mathrm{sEVs}$ mixed with $\mathrm{Ag}-\mathrm{TiO}_{2}$ nanoparticles was examined using a Tecnai G2 $20 \times$-Twin type instrument (FEI), operating at an acceleration voltage of $200 \mathrm{kV}$. For TEM measurements the samples were dropped on a grid (carbon film with 200 Mesh coper grids; CF200-Cu, Electron Microscopy Sciences) and dried.

miRNA analysis of $s E V s$. Pellets of sEVs were subjected to miRNA isolation using the NucleoSpin miRNA isolation kit (Macherey-Nagel) according to the manufacturer's instructions. Sequencing was performed using SOLiD Total RNA-Seq lit for Small RNA Libraries (Applied Biosystems) based on the manufacturer's protocol. Purification was performed on 10\% TBE-Urea gels stained with Sybr Gold nucleic acid gel stain (both from Invitrogen). Final purification was performed using PureLink PCR Micro Kit (Invitrogen). Final libraries were quality checked using High Sensitivity DNA kit on Bioanalyzer (Agilent Technologies). Concentration of the libraries was determined by the SOLiD Library TaqMan Quantitation Kit (Life Technologies). Each library was clonally amplified on SOLiD P1 DNA Beads by emulsion PCR (ePCR). Emulsions were broken using butanol, and ePCR beads were enriched for template-positive beads by hybridization with magnetic enrichment beads. Template-enriched beads were extended at the $3^{\prime}$ end in the presence of terminal transferase and $3^{\prime}$ bead linker. Beads with the clonally amplified DNA were deposited onto SOLiD sequencing slide and sequenced on SOLiD 5500xl instrument using the 50-base sequencing chemistry.

Bioinformatics analysis of raw data, quality assessment, read trimming, read mapping and miRNA expression profiling was carried out in CLC Genomics Workbench 8.0.2 (Qiagen Bioinformatics) using annotated Mus musculus miRNA sequences according to the miRBase release 21 as a mapping reference. Only miRNAs with $\geq 10$ read counts were accepted.

Results of sequencing were validated by qPCR on 3 selected miRNAs, mmu-miR-16-5p, mmu-miR-125b-5p, mmu-miR-29a-3p. Intact total RNA - including miRNA - were prepared from sEV isolates by miRNA Miniprep System (Promega) according to the manufacturer's instructions. Then, $70 \mathrm{ng}$ of each sample were reverse transcribed using microRNA cDNA synthesis kit (Sigma-Aldrich). The qPCR reactions were performed on PikoReal Real-Time PCR System (Thermo Scientific) using SYBR Green chemistry and commercially available miRNA specific primers (Sigma-Aldrich). Cq values of each miRNA were normalized by U6 endogenous controls in all samples and expression levels were calculated using -ddCt method.

Proteome analysis of sEVs. Detailed LC-MS/MS analysis of sEVs is described in the Supplementary Methods. Briefly, $25 \mu \mathrm{g}$ of vesicular proteins were separated by SDS-PAGE and stained with Coomassie blue. Then, each lane was cut to 12 equal bands and subjected to an in-gel trypsinisation procedure. The extracted peptides were analysed on an LTQ-Orbitrap Elite (Thermo Scientific) mass spectrometer on-line coupled with a nanoHPLC (nanoAcquity, Waters) system. Searchable peaklists were extracted using Proteome Discoverer 1.4 (Thermo Scientific) and subjected to database search on our in-house Protein Prospector 5.14.1 search engine against the Mus musculus and Bos taurus protein sequences of the Uniprot (UniProtKB.06.11.2014) database completed with human keratins and pig trypsin, altogether 106,330 protein sequences were searched. Protein identification was accepted if the protein was identified with $\geq 3$ unique peptides, but peptides with identical bovine and mouse sequence were excluded. FDR values were less than $1 \%$ in all cases. Results were validated by Western blot (described in the Supplementary Methods).

Bioinformatics analysis. Normalised miRNA and protein data derived from the LC-MS/MS and SOLiD sequencing were analysed by the Ingenuity Pathway Analysis (IPA, Qiagen Bioinformatics). First, we used the 'Core Analysis' feature to reveal functional differences between the five sEV groups, where 'Top 5 canonical pathways' and 'Top 5 molecular and cellular functions' (Fig. 4a,b) were obtained. Secondly, using the 'Comparison Analysis' feature, we created a heatmap containing 'Biofunctions', which had relevance in melanoma and $>5$ - $\log (\mathrm{p}$-value). This heatmap was divided into four parts based on biological relevance; intracellular, cellular, systemic and immune processes are displayed in separated panels (Fig. 4c-f). Thirdly, some 'Biofunctions' were chosen for further investigation to reveal the regulatory effects of sEVs on them. Using the 'Grow tool', the upstream interacting vesicular molecules were identified for the selected 'Biofunctions' for each sEV group. Then, using the 'Molecule Activity Predictor' (MAP) tool, we could reveal the activation or inhibitory effects of each sEV group for each 'Biofunction' (Figs. 5-8a). Through these in silico analysis, we could model the effects of the different sEVs in the recipient cells in spite of their molecular complexity. Figures were edited in the IPA 'Path Designer' and completed with Excel diagrams. For all IPA analyses, the confidence level was set to 'Experimentally observed' enabling literature data-based analysis, but not unproven predictions. 
Exposures of MSCs and B16F1 cells to the sEVs. To avoid additional effects of changing conditions, cells were exposed to sEVs in their standard, complete media before each of the following functional assays. Briefly, cells were treated with $200 \mu \mathrm{g} / \mathrm{ml} \mathrm{sEV}$ suspensions, or PBS as a negative control for $24 \mathrm{~h}, 48 \mathrm{~h}$ or $72 \mathrm{~h}$. For longer incubation times, treatments were repeated in every $24 \mathrm{~h}$.

Ki-67 expression analysis of MSCs. MSCs exposed to sEVs for $24 \mathrm{~h}$ and $72 \mathrm{~h}$ were fixed in $4 \%$ paraformaldehyde for $10 \mathrm{~min}$ at room temperature (RT) for immunocytochemistry. Then, cells were permeabilised with $0.1 \%$ Triton X-100 and non-specific antibody binding was blocked with 5\% BSA. We applied direct labelling using anti-mouse/rat Ki-67 monoclonal antibody conjugated to eFluor 615 dye (1:400, eBioScience) in 1.2\% BSA overnight at $4^{\circ} \mathrm{C}$. Nuclear counterstaining was performed with DAPI for $15 \mathrm{~min}$ at RT. Cells were washed 3 times with PBS for $5 \mathrm{~min}$ between each step. Finally, the cells were covered by Fluoromount-G (SouthernBiotech) and cover glasses. Fluorescent images were taken by the Operetta high content screening system (PerkinElmer) and analysed by an image analysis and machine learning software (SCT Analyzer 1.0) developed by the Single-Cell Technologies $\operatorname{Ltd}^{62}$. Our pipeline was comprised of cell segmentation, feature extraction and machine learning modules. K-means algorithm was used for the nuclei segmentation based on the DAPI signal, then we extracted the eFluor 615 signal-related features, i.e. max intensity, min intensity, mean intensity, median intensity, SD intensity for the generated nuclei masks. We established a training set with two classes for the Ki-67 positive and the negative cells. This training set, containing 100 objects in both classes was validated by the implemented k-fold cross-validation. For machine learning, we used the Multi-Layer Perceptron (MLP) method. The Ki-67 expression analysis was repeated 4 times and the applied methods enabled to analyse a total of 159,596 cells.

Cell counting. MSC cultures in 384-well plates were exposed to sEVs for $24 \mathrm{~h}$ and $72 \mathrm{~h}$, then fixed in $4 \%$ paraformaldehyde for $10 \mathrm{~min}$ at RT and stained with $1 \mu \mathrm{g} / \mathrm{ml}$ DAPI for $15 \mathrm{~min}$ at RT. Images were acquired from whole wells using a TCS SP8 microscope (Leica Microsystems) in fluorescent mode, followed by an analysis using the SCT Analyzer 1.0 machine learning software. The experiment was repeated 3 times.

Cell cycle analysis. Changes in the cell cycle dynamics of sEV-exposed B16F1 cells were analysed using the Cell-Clock cell cycle assay (Biocolor Ltd) according to the assay protocol. This assay can be used to distinguish the four major phases of the mammalian cell cycle using a vital redox dye, which is yellow, green or dark blue in G1, S/G2, and M phase cells, respectively. After staining, cells were photographed using an Axiovert S100 microscope (Zeiss) equipped by a Nikon D5000 camera. Images were analysed by the ImageJ software to determine the percentage of cells in each cell cycle phase. The experiment was performed with 4 repeats.

Wound healing assay. Alterations of the migration capabilities of sEV-exposed B16F1 and bEnd. 3 cells were assessed by scratch assay. Nearly confluent monolayers of cells were scratch wounded using a sterile $200 \mu \mathrm{l}$ pipette tip, washed 3 times with culture media to remove cellular debris, then treated with $200 \mu \mathrm{g} / \mathrm{ml} \mathrm{sEV}$ suspensions or PBS in fresh complete media. Wound closure was followed until the cell-free area decreased below $10 \%$ in at least 1 sample, when images were taken by an inverted microscope (Zeiss, Axiovert S100) equipped by a Nikon D5000 camera. Grey-scaled images were analysed using the MRI Wound Healing Tool in the ImageJ software. The experiment was repeated 8 times for B16F1 cells, and 4 times for bEnd. 3 cells.

Analysis of microtissue generation. Effects of different sEVs on cell-cell contact and cell-ECM interactions was examined on MSC-B16F1 and MEF-B16F1 co-cultures using a simplified 3D tumour matrix model to better represent the in vivo conditions, than 2D cultures. Equal number of MSCs or MEFs and B16F1 cells were seeded to 96-well GravityPLUS hanging drop plates (InSphero AG) in sEV- or PBS-containing media $(5,000$ cell $/ 40 \mu \mathrm{l} /$ well). Microtissue generation was followed for $72 \mathrm{~h}$ and images were acquired in every $24 \mathrm{~h}$ using an Axiovert S100 microscope (Zeiss) equipped by a Nikon D5000 camera. To quantify differences in size and shape between the microtissues, $72 \mathrm{~h}$ images were grey scaled and analysed by the AnaSP software ${ }^{79}$. Measured parameters of microtissues were the equivalent diameter, major diameter through centroid, minor diameter through centroid, convexity, solidity, sphericity, area, perimeter and volume. The experiments were repeated 3 times.

Statistical analysis. Since the homogeneity of variances assumption of the ANOVA had not met with our data, statistical analyses were performed by the Welch's ANOVA test with Tukey's HSD post-hoc test (Alpha $=0.05)$ using a Microsoft Excel add-in, the Real Statistics Resource Pack software (Release 5.4). Copyright (2013-2018) Charles Zaiontz (www.real-statistics.com). Diagrams were prepared in GraphPad Prism 5.03. All average values represent mean $\pm S D$ and number of asterisk denote minimum statistical significance, i.e. ${ }^{*} \mathrm{p}<0.05$, $* * \mathrm{p}<0.01$ and $* * * \mathrm{p}<0.001$ on figures. Exact p-values are indicated in the text, when it is necessary. Figure 1 was created with BioRender.com.

\section{Data availability}

All datasets generated during the current study are available from the corresponding author upon reasonable request.

Received: 26 March 2019; Accepted: 23 September 2019;

Published online: 25 October 2019

\section{References}

1. Kotrbová, A. et al. TEM Exosome Analyzer: a computer-assisted software tool for quantitative evaluation of extracellular vesicles in transmission electron microscopy images. J Extracell Vesicles. 8, 1560808, https://doi.org/10.1080/20013078.2018.1560808 (2019).

2. Yáñez-Mó, M. et al. Biological properties of extracellular vesicles and their physiological functions. J Extracell Vesicles. 4, 27066, https://doi.org/10.3402/jev.v4.27066 (2015). 
3. Kolenda, T. et al. Tumor microenvironment - Unknown niche with powerful therapeutic potential. Rep Pract Oncol Radiother. 23, 143-153, https://doi.org/10.1016/j.rpor.2018.01.004 (2018).

4. Meehan, K. \& Vella, L. J. The contribution of tumour-derived exosomes to the hallmarks of cancer. Crit Rev Clin Lab Sci. 53, 121-131, https://doi.org/10.3109/10408363.2015.1092496 (2016)

5. Javeed, N. \& Mukhopadhyay, D. Exosomes and their role in the micro-/macro-environment: a comprehensive review. J Biomed Res. 31, 386-394, https://doi.org/10.7555/JBR.30.20150162 (2017).

6. Tkach, M. \& Théry, C. Communication by Extracellular Vesicles: Where We Are and Where We Need to Go. Cell. 164, 1226-1232, https://doi.org/10.1016/j.cell.2016.01.043 (2016).

7. Whiteside, T. L. Tumor-Derived Exosomes and Their Role in Cancer Progression. Adv Clin Chem. 74, 103-141, https://doi. org/10.1016/bs.acc.2015.12.005 (2016).

8. Braeuer, R. R. et al. Why is melanoma so metastatic? Pigment Cell Melanoma Res. 27, 19-36, https://doi.org/10.1111/pcmr.12172 (2014).

9. Matsumoto, A. et al. Accelerated growth of B16BL6 tumor in mice through efficient uptake of their own exosomes by B16BL6 cells. Cancer Sci. 108, 1803-1810, https://doi.org/10.1111/cas.13310 (2017).

10. Guo, D. et al. RAB27A promotes melanoma cell invasion and metastasis via regulation of pro-invasive exosomes. Int J Cancer. 144, 3070-3085, https://doi.org/10.1002/ijc.32064 (2019).

11. Li, J. et al. Blockage of transferred exosome-shuttled miR-494 inhibits melanoma growth and metastasis. J Cell Physiol. 234, 15763-15774, https://doi.org/10.1002/jcp.28234 (2019).

12. Isola, A. L., Eddy, K., Zembrzuski, K., Goydos, J. S. \& Chen, S. Exosomes released by metabotropic glutamate receptor 1 (GRM1) expressing melanoma cells increase cell migration and invasiveness. Oncotarget. 9, 1187-1199, https://doi.org/10.18632/ oncotarget.23455 (2017).

13. Xiao, D. et al. Melanoma cell-derived exosomes promote epithelial-mesenchymal transition in primary melanocytes through paracrine/autocrine signaling in the tumor microenvironment. Cancer Lett. 376, 318-327, https://doi.org/10.1016/j. canlet.2016.03.050 (2016)

14. Hood, J. L., San, R. S. \& Wickline, S. A. Exosomes released by melanoma cells prepare sentinel lymph nodes for tumor metastasis. Cancer Res. 71, 3792-3801, https://doi.org/10.1158/0008-5472.CAN-10-4455 (2011).

15. Shu, S. L. et al. Metabolic reprogramming of stromal fibroblasts by melanoma exosome microRNA favours a pre-metastatic microenvironment. Sci Rep. 8, 12905, https://doi.org/10.1038/s41598-018-31323-7 (2018)

16. Lin, L. Y. et al. Tumour cell-derived exosomes endow mesenchymal stromal cells with tumour-promotion capabilities. Oncogene. 35, 6038-6042, https://doi.org/10.1038/onc.2016.131 (2016).

17. Peinado, H. et al. Melanoma exosomes educate bone marrow progenitor cells toward a pro-metastatic phenotype through MET. Nat Med. 18, 883-891, https://doi.org/10.1038/nm.2753 (2012).

18. Zhou, X. et al. Melanoma cell-secreted exosomal miR-155-5p induce proangiogenic switch of cancer-associated fibroblasts via SOCS1/JAK2/STAT3 signaling pathway. J Exp Clin Cancer Res. 37, 242, https://doi.org/10.1186/s13046-018-0911-3 (2018).

19. Hood, J. L. Melanoma exosome induction of endothelial cell GM-CSF in pre-metastatic lymph nodes may result in different M1 and M2 macrophage mediated angiogenic processes. Med Hypotheses. 94, 118-122, https://doi.org/10.1016/j.mehy.2016.07.009 (2016).

20. Boussadia, Z. et al. Acidic microenvironment plays a key role in human melanoma progression through a sustained exosome mediated transfer of clinically relevant metastatic molecules. J Exp Clin Cancer Res. 37, 245, https://doi.org/10.1186/s13046-0180915-z (2018).

21. Wozniak, M., Peczek, L., Czernek, L. \& Düchler, M. Analysis of the miRNA Profiles of Melanoma Exosomes Derived Under Normoxic and Hypoxic Culture Conditions. Anticancer Res. 37, 6779-6789, https://doi.org/10.21873/anticanres.12138 (2017).

22. Cesi, G. et al. A new ALK isoform transported by extracellular vesicles confers drug resistance to melanoma cells. Mol Cancer. 17, 145, https://doi.org/10.1186/s12943-018-0886-x (2018).

23. Théry, C. et al. Minimal information for studies of extracellular vesicles 2018 (MISEV2018): a position statement of the International Society for Extracellular Vesicles and update of the MISEV2014 guidelines. J Extracell Vesicles. 7, 1535750, https://doi.org/10.1080/ 20013078.2018.1535750 (2018).

24. König, L. et al. Elevated levels of extracellular vesicles are associated with therapy failure and disease progression in breast cancer patients undergoing neoadjuvant chemotherapy. Oncoimmunology. 7, e1376153, https://doi.org/10.1080/2162402X.2017.1376153 (2017).

25. Osti, D. et al. Clinical Significance of Extracellular Vesicles in Plasma from Glioblastoma Patients. Clin Cancer Res. 25, 266-276, https://doi.org/10.1158/1078-0432.CCR-18-1941 (2019).

26. Shaked, Y. Balancing efficacy of and host immune responses to cancer therapy: the yin and yang effects. Nat Rev Clin Oncol. 13, 611-626, https://doi.org/10.1038/nrclinonc.2016.57 (2016).

27. Keklikoglou, I. et al. Chemotherapy elicits pro-metastatic extracellular vesicles in breast cancer models. Nat Cell Biol. 21, 190-202, https://doi.org/10.1038/s41556-018-0256-3 (2019).

28. Lee, S. et al. Immunogenic Effect of Hyperthermia on Enhancing Radiotherapeutic Efficacy. Int J Mol Sci. 19, E2795, https://doi. org/10.3390/ijms19092795 (2018)

29. Mahmood, J. et al. Immunotherapy, Radiotherapy, and Hyperthermia: A Combined Therapeutic Approach in Pancreatic Cancer Treatment. Cancers (Basel). 10, E469, https://doi.org/10.3390/cancers10120469 (2018).

30. Tallósy, S. P. et al. Adhesion and inactivation of Gram-negative and Gram-positive bacteria on photoreactive $\mathrm{TiO}_{2} /$ polymer and $\mathrm{Ag}-\mathrm{TiO}_{2}$ /polymer nanohybrid films. Appl Surf Sci. 371, 139-150, https://doi.org/10.1016/j.apsusc.2016.02.202 (2016).

31. Tallósy, S. P. et al. Investigation of the antibacterial effects of silver-modified $\mathrm{TiO}_{2}$ and $\mathrm{ZnO}$ plasmonic photocatalysts embedded in polymer thin films. Environ Sci Pollut Res Int. 21, 11155-11167, https://doi.org/10.1007/s11356-014-2568-6 (2014).

32. Szweda, P. et al. Essential Oils, Silver Nanoparticles and Propolis as Alternative Agents Against Fluconazole Resistant Candida albicans, Candida glabrata and Candida krusei Clinical Isolates. Indian J Microbiol. 55, 175-183, https://doi.org/10.1007/s12088014-0508-2 (2015).

33. Ahamed, M., Khan, M. A. M., Akhtar, M. J., Alhadlaq, H. A. \& Alshamsan, A. Ag-doping regulates the cytotoxicity of $\mathrm{TiO}_{2}$ nanoparticles via oxidative stress in human cancer cells. Sci Rep. 7, 17662, https://doi.org/10.1038/s41598-017-17559-9 (2017).

34. Tani, F. et al. Surface expression of a C-terminal alpha-helix region in heat shock protein 72 on murine LL/2 lung carcinoma can be recognized by innate immune sentinels. Mol Immunol. 46, 1326-1339, https://doi.org/10.1016/j.molimm.2008.11.020 (2009).

35. Veres, Á. et al. Silver and gold modified plasmonic $\mathrm{TiO}_{2}$ hybrid films for photocatalytic decomposition of ethanol under visible light. Catal Today. 181, 156-162, https://doi.org/10.1016/j.cattod.2011.05.028 (2012).

36. Hawley R.J., Kozlovac J.P. Decontamination. In: Lindler L.E., Lebeda F. J., Korch G. W. (eds) Biological Weapons Defense. Infectious Disease. Humana Press, https://doi.org/10.1385/1-59259-764-5:333 (2005).

37. Kucharzewska, P. \& Belting, M. Emerging roles of extracellular vesicles in the adaptive response of tumour cells to microenvironmental stress. J Extracell Vesicles. 2, 10.3402/jev.v2i0.20304, https://doi.org/10.3402/jev.v2i0.20304, (2013).

38. King, H. W., Michael, M. Z. \& Gleadle, J. M. Hypoxic enhancement of exosome release by breast cancer cells. BMC Cancer. 12, 421, https://doi.org/10.1186/1471-2407-12-421 (2012).

39. Parolini, I. et al. Microenvironmental pH is a key factor for exosome traffic in tumor cells. J Biol Chem. 284, 34211-34222, https:// doi.org/10.1074/jbc.M109.041152 (2009). 
40. Hedlund, M., Nagaeva, O., Kargl, D., Baranov., V. \& Mincheva-Nilsson, L. Thermal- and oxidative stress causes enhanced release of NKG2D ligand-bearing immunosuppressive exosomes in leukemia/lymphoma T and B cells. PLoS One 6, e16899, https://doi. org/10.1371/journal.pone.0016899 (2011).

41. Jelonek, K., Widlak, P. \& Pietrowska, M. The Influence of Ionizing Radiation on Exosome Composition, Secretion and Intercellular Communication. Protein Pept Lett. 23, 656-663, https://doi.org/10.2174/0929866523666160427105138 (2016).

42. Lv, L. H. et al. Anticancer drugs cause release of exosomes with heat shock proteins from human hepatocellular carcinoma cells that elicit effective natural killer cell antitumor responses in vitro. J Biol Chem. 287, 15874-15885, https://doi.org/10.1074/jbc. M112.340588 (2012).

43. Yang, Y., Chen, Y., Zhang, F., Zhao, Q. \& Zhong, H. Increased anti-tumour activity by exosomes derived from doxorubicin-treated tumour cells via heat stress. Int J Hyperthermia. 31, 498-506, https://doi.org/10.3109/02656736.2015.1036384 (2015).

44. Harmati, M. et al. Stressors alter intercellular communication and exosome profile of nasopharyngeal carcinoma cells. J Oral Pathol Med. 46, 259-266, https://doi.org/10.1111/jop.12486 (2017).

45. Zapata-Benavides, P. et al. WT1 silencing by RNAi synergizes with chemotherapeutic agents and induces chemosensitization to doxorubicin and cisplatin in B16F10 murine melanoma cells. Oncology letters. 3, 751-755, https://doi.org/10.3892/ol.2012.578 (2012)

46. Veres, Á. et al. Photocatalytic performance of silver-modified $\mathrm{TiO}_{2}$ embedded in poly(ethyl-acrylate-co-methyl metacrylate) matrix. Colloid Polym Sci. 292, 207-217, https://doi.org/10.1007/s00396-013-3063-1 (2014).

47. Mutschelknaus, L. et al. Radiation alters the cargo of exosomes released from squamous head and neck cancer cells to promote migration of recipient cells. Sci Rep. 7, 12423, https://doi.org/10.1038/s41598-017-12403-6 (2017).

48. Wozniak, M., Peczek, L., Czernek, L. \& Düchler, M. Analysis of the miRNA Profiles of Melanoma Exosomes Derived Under Normoxic and Hypoxic Culture Conditions. Anticancer Res. 37, 6779-6789, https://doi.org/10.21873/anticanres.12138 (2017).

49. Lazar, I. et al. Proteome characterization of melanoma exosomes reveals a specific signature for metastatic cell lines. Pigment Cell Melanoma Res. 28, 464-475, https://doi.org/10.1111/pcmr.12380 (2015).

50. Warburg, O. On the origin of cancer cells. Science 123, 309-314, https://doi.org/10.1126/science.123.3191.309 (1956).

51. Vazquez, A., Liu, J., Zhou, Y. \& Oltvai, Z. N. Catabolic efficiency of aerobic glycolysis: the Warburg effect revisited. BMC Syst Biol. 4, 58, https://doi.org/10.1186/1752-0509-4-58 (2010).

52. Hessvik, N. P. \& Llorente, A. Current knowledge on exosome biogenesis and release. Cell Mol Life Sci. 75, 193-208, https://doi. org/10.1007/s00018-017-2595-9 (2018).

53. Alaseem, A. et al. Matrix Metalloproteinases: A challenging paradigm of cancer management. Semin Cancer Biol. 56, 100-115, https://doi.org/10.1016/j.semcancer.2017.11.008 (2019).

54. Jabłońska-Trypuć, A., Matejczyk, M. \& Rosochacki, S. Matrix metalloproteinases (MMPs), the main extracellular matrix (ECM) enzymes in collagen degradation, as a target for anticancer drugs. J Enzyme Inhib Med Chem. 31, 177-183, https://doi.org/10.3109/ 14756366.2016 .1161620 (2016).

55. Zheng, Q., Ye, J. \& Cao, J. Translational regulator eIF2 $\alpha$ in tumor. Tumour Biol. 35, 6255-6264, https://doi.org/10.1007/s13277-0141789-0 (2014).

56. Tai, Y. L., Chen, K. C., Hsieh, J. T. \& Shen, T. L. Exosomes in cancer development and clinical applications. Cancer Sci. 109, 2364-2374, https://doi.org/10.1111/cas.13697 (2018).

57. Saleem, S. N. \& Abdel-Mageed, A. B. Tumor-derived exosomes in oncogenic reprogramming and cancer progression. Cell Mol Life Sci. 72, 1-10, https://doi.org/10.1007/s00018-014-1710-4 (2014).

58. Becker, A. et al. Extracellular Vesicles in Cancer: Cell-to-Cell Mediators of Metastasis. Cancer Cell 30, 836-848, https://doi. org/10.1016/j.ccell.2016.10.009 (2016).

59. Penning, T. M. \& Drury, J. E. Human aldo-keto reductases: Function, gene regulation, and single nucleotide polymorphisms. Arch Biochem Biophys. 464, 241-250, https://doi.org/10.1016/j.abb.2007.04.024 (2007).

60. Tammali, R., Reddy, A. B., Srivastava, S. K. \& Ramana, K. V. Inhibition of aldose reductase prevents angiogenesis in vitro and in vivo. Angiogenesis. 14, 209-221, https://doi.org/10.1007/s10456-011-9206-4 (2011).

61. Sharma, M. et al. Developmental Competence of Buffalo (Bubalus bubalis) Pluripotent Embryonic Stem Cells Over Different Homologous Feeder Layers and the Comparative Evaluation with Various Extracellular Matrices. Int J Stem Cells. 6, 26-36, https:// doi.org/10.15283/ijsc.2013.6.1.26 (2013)

62. Toth, T. et al. Environmental properties of cells improve machine learning-based phenotype recognition accuracy. Sci Rep. 8, 10085, https://doi.org/10.1038/s41598-018-28482-y (2018).

63. Baglio, S. R. et al. Blocking Tumor-Educated MSC Paracrine Activity Halts Osteosarcoma Progression. Clin Cancer Res. 23, 3721-3733, https://doi.org/10.1158/1078-0432.CCR-16-2726 (2017).

64. Cho, J. A., Park, H., Lim, E. H. \& Lee, K. W. Exosomes from breast cancer cells can convert adipose tissue-derived mesenchymal stem cells into myofibroblast-like cells. Int J Oncol. 40, 130-138, https://doi.org/10.3892/ijo.2011.1193 (2012).

65. Yamashita, A., Hakura, A. \& Inoue, H. Suppression of anchorage-independent growth of human cancer cell lines by the drs gene. Oncogene 18, 4777-4787, https://doi.org/10.1038/sj.onc.1202852 (1999).

66. Tarasov, V. et al. Differential regulation of microRNAs by p 53 revealed by massively parallel sequencing: miR-34a is a p53 target that induces apoptosis and G1-arrest. Cell Cycle. 6, 1586-1593, https://doi.org/10.4161/cc.6.13.4436 (2007).

67. Ji, Q. et al. MicroRNA miR-34 inhibits human pancreatic cancer tumor-initiating cells. PLoS One. 4, e6816, https://doi.org/10.1371/ journal.pone.0006816 (2009)

68. Zhao, L., Liu, W., Xiao, J. \& Cao, B. The role of exosomes and "exosomal shuttle microRNA" in tumorigenesis and drug resistance. Cancer Lett. 356, 339-346, https://doi.org/10.1016/j.canlet.2014.10.027 (2015).

69. Bar-On, O., Shapira, M. \& Hershko, D. D. Differential effects of doxorubicin treatment on cell cycle arrest and Skp2 expression in breast cancer cells. Anticancer Drugs. 18, 1113-1121 https://doi.org/10.1097/CAD.0b013e3282ef4571 (2007).

70. Lüpertz, R., Wätjen, W., Kahl, R. \& Chovolou, Y. Dose- and time-dependent effects of doxorubicin on cytotoxicity, cell cycle and apoptotic cell death in human colon cancer cells. Toxicology. 271, 115-121, https://doi.org/10.1016/j.tox.2010.03.012 (2010).

71. Hibino, S. et al. Identification of an active site on the laminin alpha5 chain globular domain that binds to CD44 and inhibits malignancy. Cancer Res. 64, 4810-4816, https://doi.org/10.1158/0008-5472.CAN-04-0129 (2004).

72. Lee, D. J. et al. Peroxiredoxin- 2 represses melanoma metastasis by increasing E-Cadherin/ $\beta$-Catenin complexes in adherens junctions. Cancer Res. 73, 4744-4757, https://doi.org/10.1158/0008-5472.CAN-12-4226 (2013).

73. Stampolidis, P., Ullrich, A. \& Iacobelli, S. LGALS3BP, lectin galactoside-binding soluble 3 binding protein, promotes oncogenic cellular events impeded by antibody intervention. Oncogene. 34, 39-52, https://doi.org/10.1038/onc.2013.548 (2015)

74. Friedl, P. \& Wolf, K. Tumour-cell invasion and migration: diversity and escape mechanisms. Nat Rev Cancer. 3, 362-374, https://doi. org/10.1038/nrc1075 (2003).

75. Huang, Z., Yang, M., Li, Y., Yang, F. \& Feng, Y. Exosomes Derived from Hypoxic Colorectal Cancer Cells Transfer Wnt4 to Normoxic Cells to Elicit a Prometastatic Phenotype. Int J Biol Sci. 14, 2094-2102, https://doi.org/10.7150/ijbs.28288 (2018).

76. Lamalice, L., Le Boeuf, F. \& Huot, J. Endothelial cell migration during angiogenesis. Circ Res. 100, 782-794, https://doi. org/10.1161/01.RES.0000259593.07661.1e (2007).

77. Pan, S. et al. Extracellular Alix regulates integrin-mediated cell adhesions and extracellular matrix assembly. EMBO J. 27, 2077-2090, https://doi.org/10.1038/emboj.2008.134 (2008). 
78. Piccinini, F., Santis, I. \& Bevilacqua, A. Advances in cancer modeling: fluidic systems for increasing representativeness of large 3D multicellular spheroids. Biotechniques. 65, 312-314, https://doi.org/10.2144/btn-2018-0153 (2018).

79. Piccinini, F. AnaSP: a software suite for automatic image analysis of multicellular spheroids. Comput Methods Programs Biomed. 119, 43-52, https://doi.org/10.1016/j.cmpb.2015.02.006 (2015).

80. Crawford, S., Diamond, D., Brustolon, L. \& Penarreta, R. Effect of increased extracellular Ca ${ }^{++}$on microvesicle production and tumor spheroid formation. Cancer Microenviron. 4, 93-103, https://doi.org/10.1007/s12307-010-0049-0 (2010).

81. Huang, B. W. \& Gao, J. Q. Application of 3D cultured multicellular spheroid tumor models in tumor-targeted drug delivery system research. J Control Release. 270, 246-259, https://doi.org/10.1016/j.jconrel.2017.12.005 (2018).

82. Samuel, P. et al. Cisplatin induces the release of extracellular vesicles from ovarian cancer cells that can induce invasiveness and drug resistance in bystander cells. Philos Trans R Soc Lond B Biol Sci. 373, 20170065, https://doi.org/10.1098/rstb.2017.0065 (2018).

83. Santos, J. C. et al. Exosome-mediated breast cancer chemoresistance via miR-155 transfer. Sci Rep. 8, 829, https://doi.org/10.1038/ s41598-018-19339-5 (2018).

\section{Acknowledgements}

The authors thank for any help of other researchers in the Biological Research Centre of the Hungarian Academy of Sciences, namely Lilla Pinter, Ede Migh and Arpad Balind for their technical assistance, Ferenc Kovacs, Andras Kriston, Norbert Bara for developing the SCT Analyzer 1.0, Zsolt Szegletes for the AFM images, Andras Kincses for the DLS measurements of sEVs, Maria Deli for providing the bEnd. 3 cell line and members of the Laboratory of Tumor Immunology and Pharmacology for making available their tissue culture facility. We would like to thank Zoltan Arpad Varadi for the IT support. This work was supported by GINOP-2.3.2-15-201600015; GINOP-2.2.1-15-2017-00052 and NKFI-6-K funding scheme (11493 project). The Ministry of Human Capacities, Hungary grant 20391-3/2018/FEKUSTRAT and the University of Szeged Open Access Fund is also acknowledged. Krisztina Buzas and Tibor Pankotai are supported by János Bolyai Research Scholarship of the Hungarian Academy of Sciences, Krisztina Buzas is also supported by the ÚNKP-19-4 New National Excellence Program of the Ministry for Innovation and Technology.

\section{Author contributions}

K.B. supervised the research, conceived and designed the experiments and contributed to the writing of the paper; K.B. and P.H. provided resources to this study; M.H. contributed to the experimental design; performed most of the experiments; analysed data and wrote the manuscript. E.Gy.-S. contributed to the experimental work, including the Western blot analyses and miRNA isolation, G.D. contributed to the experimental work, including the Cell-Clock cell cycle assay, fluorescence spectroscopy and to the data analysis, L.J. and I.D. synthetized the Ag$\mathrm{TiO}_{2}$ photocatalyst particles and performed their DLS measurements and TEM, O.S. performed the NTA, E.H.-G. performed the proteomic analysis, I.N performed the miRNA analysis, A.F. performed the SEM, Zs.U. performed the qPCR analyses of miRNAs, M.K. performed the high-content screening and contributed to the data analysis, F.P. contributed to the developing of the 3D cell culture model and the data analysis, T.P. and T.B. provided critical feedback to the study; all authors revised the manuscript.

\section{Competing interests}

The authors declare no competing interests.

\section{Additional information}

Supplementary information is available for this paper at https://doi.org/10.1038/s41598-019-51778-6.

Correspondence and requests for materials should be addressed to K.B.

Reprints and permissions information is available at www.nature.com/reprints.

Publisher's note Springer Nature remains neutral with regard to jurisdictional claims in published maps and institutional affiliations.

Open Access This article is licensed under a Creative Commons Attribution 4.0 International License, which permits use, sharing, adaptation, distribution and reproduction in any medium or format, as long as you give appropriate credit to the original author(s) and the source, provide a link to the Creative Commons license, and indicate if changes were made. The images or other third party material in this article are included in the article's Creative Commons license, unless indicated otherwise in a credit line to the material. If material is not included in the article's Creative Commons license and your intended use is not permitted by statutory regulation or exceeds the permitted use, you will need to obtain permission directly from the copyright holder. To view a copy of this license, visit http://creativecommons.org/licenses/by/4.0/.

(C) The Author(s) 2019 


\section{SUPPLEMENTARY INFORMATION}

\section{Small extracellular vesicles convey the stress-induced adaptive responses of melanoma cells}

Maria Harmati ${ }^{1,2}$, Edina Gyukity-Sebestyen ${ }^{1,2}$, Gabriella Dobra ${ }^{1,2}$, Laszlo Janovak ${ }^{3}$, Imre Dekany ${ }^{3}$, Okay Saydam ${ }^{4}$, Eva Hunyadi-Gulyas ${ }^{5}$, Istvan Nagy ${ }^{6}$, Attila Farkas ${ }^{7}$, Tibor Pankotai ${ }^{8,9}$, Zsuzsanna Ujfaludi ${ }^{8,9}$, Peter Horvath $^{1,10}$, Filippo Piccinini ${ }^{11}$, Maria Kovacs ${ }^{1}$, Tamas Biro ${ }^{12,13}$, Krisztina Buzas ${ }^{1,9}$

${ }^{1}$ Laboratory of Microscopic Image Analysis and Machine Learning, Institute of Biochemistry, Biological Research Centre of the Hungarian Academy of Sciences, Szeged, Hungary

${ }^{2}$ Doctoral School of Interdisciplinary Medicine, Faculty of Medicine, University of Szeged, Szeged, Hungary

${ }^{3}$ Interdisciplinary Excellence Centre, Department of Physical Chemistry and Materials Science, University of Szeged, Szeged, Hungary

${ }^{4}$ Department of Pediatrics, University of Minnesota, Minneapolis, USA

${ }^{5}$ Laboratory of Proteomics Research, Institute of Biochemistry, Biological Research Centre of the Hungarian Academy of Sciences, Szeged, Hungary

${ }^{6}$ Sequencing Platform, Institute of Biochemistry, Biological Research Centre of the Hungarian Academy of Sciences, Szeged, Hungary

${ }^{7}$ Laboratory of Microbial Genomics, Institute of Plant Biology, Biological Research Centre of the Hungarian Academy of Sciences, Szeged, Hungary

${ }^{8}$ Department of Biochemistry and Molecular Biology, Faculty of Science and Informatics, University of Szeged, Szeged, Hungary

${ }^{9}$ Department of Oral Biology and Experimental Dental Research, Faculty of Dentistry, University of Szeged, Szeged, Hungary

${ }^{10}$ Institute for Molecular Medicine Finland, University of Helsinki, Helsinki, Finland

${ }^{11}$ Laboratorio di Bioscienze, Istituto Scientifico Romagnolo per lo Studio e la Cura dei Tumori (IRST) IRCCS, Meldola, Italy

${ }^{12}$ Department of Immunology, Faculty of Medicine, University of Debrecen, Debrecen, Hungary

${ }^{13}$ Hungarian Centre of Excellence for Molecular Medicine, Szeged, Hungary 


\section{SUPPLEMENTARY METHODS}

\section{Proteomic analysis by LC-MS/MS}

\section{Separation and in-gel digestion of sEV proteins}

Vesicular pellets were resuspended in NP40 lysis buffer (Invitrogen) and incubated on ice for $30 \mathrm{~min}$. Protein content of sEV isolates were measured as described in the Methods section and $25 \mu \mathrm{g}$ protein of each sample was diluted in a mixture of $4 \times$ sample buffer (NuPAGE LDS Sample Buffer) and 10x reducing agent (NuPAGE Sample Reducing Agent), boiled at $96^{\circ} \mathrm{C}$ for $10 \mathrm{~min}$, immediately cooled on ice and separated in 4-12\% Bis-Tris Protein Gel (NuPAGE Novex) using an XCell SureLock Mini-Cell (Invitrogen) at $200 \mathrm{~V}$ and $0.03 \mathrm{~A}$ for $40 \mathrm{~min}$ with an electrophoresis buffer (NuPAGE MOPS SDS Running Buffer). Then, the gel was stained overnight with Coomassie blue (0.1\% Coomassie Brilliant Blue R-250 in $45 \%$ ethanol, $45 \% \mathrm{MQ}$ water and $10 \%$ acetic acid), destained with a mixture of $10 \%$ acetic acid, $30 \%$ ethanol and 60\% MQ water and washed in MQ water. Each lane was cut to 12 equal bands and subjected to in-gel digestion. Gel bands were diced to smaller pieces, and the SDS and the dye were washed out with $3 \times 50 \mu \mathrm{l} 25 \mathrm{mM}$ ammonium-bicarbonate $(A B C) / 50 \%$ Acetonitrile $(A c N)$. After reduction with DTT (1,4-dithiothreitol, Sigma; $20 \mu \mathrm{l}, 10 \mathrm{mM}$ DTT in $25 \mathrm{mM} \mathrm{ABC}$ ) at $56^{\circ} \mathrm{C}$ for $30 \mathrm{~min}$, and alkylation with IAM (iodoacetamide, Sigma; $20 \mu \mathrm{l}, 55 \mathrm{mM}$ IAM in $25 \mathrm{mM} \mathrm{ABC}$ ) at room temperature (RT) in dark for $30 \mathrm{~min}$, the gel samples were dried in a vacuum centrifuge and after that rehydrated in $20 \mu \mathrm{l}$ of trypsin (Sequencing Grade Modified Trypsin, Promega; $5 \mathrm{ng} / \mu \mathrm{l}$ in $25 \mathrm{mM} \mathrm{ABC}$ ) and incubated at $37^{\circ} \mathrm{C}$. The digestion was stopped after $4 \mathrm{~h}$ by lowering the $\mathrm{pH}$ of the buffer below 3, by adding $2 \mu \mathrm{l}$ of $10 \%$ formic acid (FA). Tryptic peptides were extracted from the gel with $3 \times 50 \mu \mathrm{l}$ of $2 \%$ FA in $50 \%$ AcN and dried. Prior mass spectrometric analysis, all samples were redissolved in $50 \mu \mathrm{l}$ of $0.1 \%$ FA.

\section{Mass spectrometry analysis and protein identification}

Samples were analysed on an LTQ-Orbitrap Elite (Thermo Scientific) mass spectrometer on-line coupled with a nanoHPLC (nanoAcquity, Waters) system. Five $\mu$ of the in-gel digests were loaded (for $3 \mathrm{~min}$ at $8 \mu \mathrm{l} / \mathrm{min}$ flow, using $0.1 \% \mathrm{FA}$ in $3 \% \mathrm{AcN}-97 \%$ water) onto a reversed phase trap column (Waters, Symmetry C18, 0.18×20 mm) and separated on a C18 reversed phase (Waters, BEH300C18 $1.7 \mu \mathrm{m}$ ) nanocolumn $(0.075 \times 200 \mathrm{~mm})$. The flow rate was $330 \mathrm{nl} / \mathrm{min}$ and a linear gradient was used from $3 \%$ to $40 \%$ solvent $B$ in 37 min (solvent $A$ was $0.1 \%$ FA in water and solvent $B$ was $0.1 \%$ FA in $A c N$ ).

The high voltage $(1.2 \mathrm{kV})$ was applied through the liquid junction between the chromatographic column and the non-coated silica nanospray emitter (NewObjective, $10 \mu \mathrm{m}$ tip ID). The mass spectrometer operated in data-dependent mode: the survey mass spectra were detected in the Orbitrap with high resolution ( $R=60 \mathrm{k} @ \mathrm{~m} / \mathrm{z}$ : 400, mass range m/z: 380-1400) and the most abundant multiply charged 20 peaks were selected for ion-trap fragmentation (NCE: 35\%; activation q: 0.25; activation time: $10 \mathrm{~ms}$; minimum signal intensity: 5000 counts). The MS/MS spectra were detected in the ion trap. Dynamic exclusion was used, the precursors were excluded for $15 \mathrm{~s}$ after the first fragmentation event.

Data analysis: searchable peaklists (mgf format) were extracted using Proteome Discoverer 1.4 (Thermo Scientific) and subjected to database search on our in-house ProteinProspector 5.14.1 search engine using the following parameters: parent ion tolerant: $5 \mathrm{ppm}$; fragment ion tolerant: $0.6 \mathrm{Da}$; Cys carbamidomethylation was set as constant and Met oxidation, cyclisation of peptide N-terminal Glu to pyroglutamic acid, protein $\mathrm{N}$ terminal acetylation were set as variable modifications. Only fully tryptic peptides were considered with maximum of 2 missed cleavage sites. The Mus musculus and Bos taurus protein sequences of the Uniprot (UniProtKB.06.11.2014) database completed with human keratins and pig trypsin, altogether 106,330 protein sequences were searched. 
For the false discovery rate (FDR) estimation, the searches were performed on the database concatenated with the randomized sequences. Protein identification was accepted if the ProteinProspector expectation value was less than 0.01 and the protein was identified with at least 3 unique peptides (expectation value less than 0.05 and score higher than 15). Peptides with identical bovine and mouse sequence were excluded also. FDR values were less than $1 \%$ in all cases.

\section{Western blot}

Protein samples were prepared and separated beside a ProSieve color protein marker (Lonza), as described above. Then proteins were transferred to an Immobilon-P PVDF membrane (Millipore) using an XCell SureLock Mini-Cell (Invitrogen) at $30 \mathrm{~V}$ and 10-170 mA for $60 \mathrm{~min}$ with a transfer buffer (NuPAGE). Blocking was performed by $5 \%$ non-fat milk in TBST buffer ( $25 \mathrm{mM}$ Tris- $\mathrm{HCl}, 150 \mathrm{mM} \mathrm{NaCl}$, $0.05 \%$ Tween-20, $\mathrm{pH}$ 7.2) for $60 \mathrm{~min}$ at RT. For detection of the vesicular markers, we used rabbit anti-mouse CD9 (1:500, LifeSpan Biosciences), rabbit anti-mouse CD63 (1:500, Santa Cruz), mouse HSP70/HSP72 monoclonal antibody (1:8000, Enzo Life Sciences, Inc.), rabbit anti-mouse Alix (1:500, Sigma-Aldrich), rabbit anti-mouse TSG101 (1:2500, Sigma-Aldrich), rabbit anti-mouse Calnexin (1:1000, Abcam) and rabbit MLANA polyclonal antibody (1:2000, ABclonal). Primary antibody labelling was performed in $1 \%$ non-fat milk in TBST buffer overnight at $4^{\circ} \mathrm{C}$. Unbound antibodies were removed by washing 3 times for $10 \mathrm{~min}$ in TBST buffer. As secondary antibodies, HRP-conjugated anti-rabbit IgG and anti-mouse IgG (R\&D Systems) were used in 1\% non-fat milk-containing TBST buffer with 60 min incubation at RT. After washing, protein bands were visualised by the WesternBright ECL detection kit (Advansta) and an Odyssey Fc imaging system (LI-COR) coupled with the Image Studio Lite 5.2 software. 
AFM
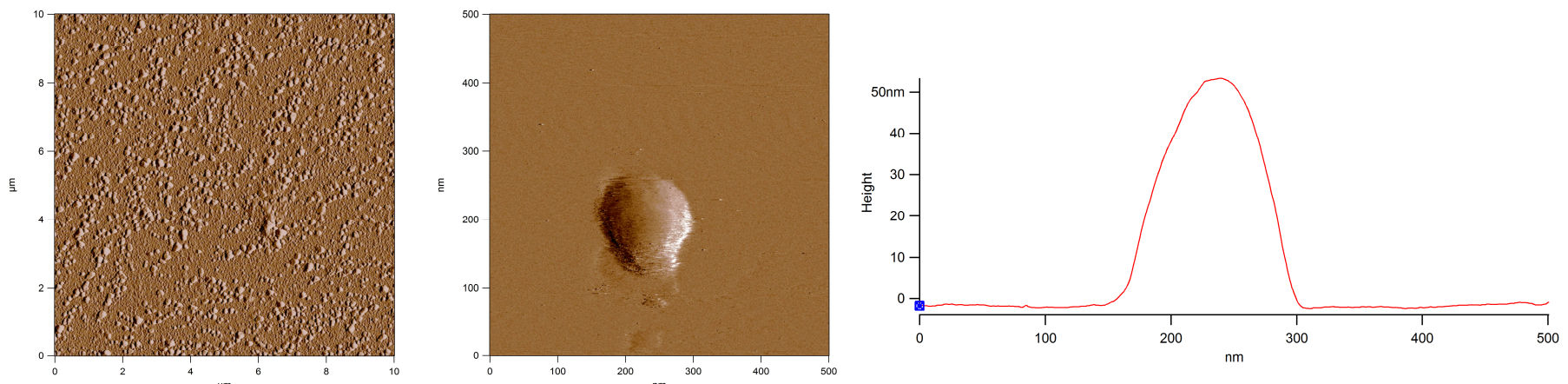

\section{DLS by Zetasizer Nano S}

Size Distribution by Intensity

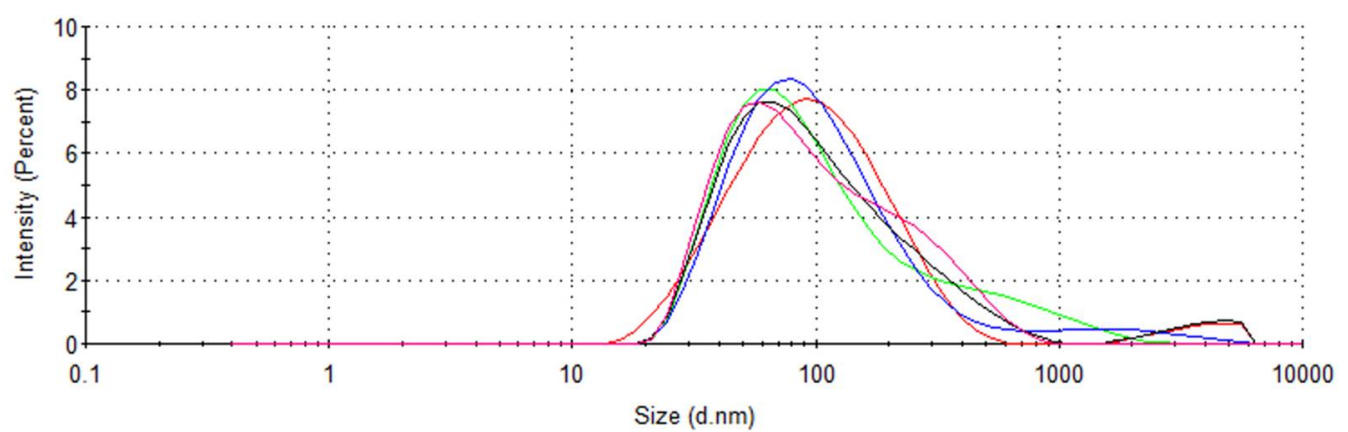

\begin{tabular}{|l|c|}
\hline & $\begin{array}{c}\text { Z-Average } \\
\text { (d.nm) }\end{array}$ \\
\hline Mean & 78.27 \\
\hline SD & 0.7796 \\
\hline
\end{tabular}

Western blot

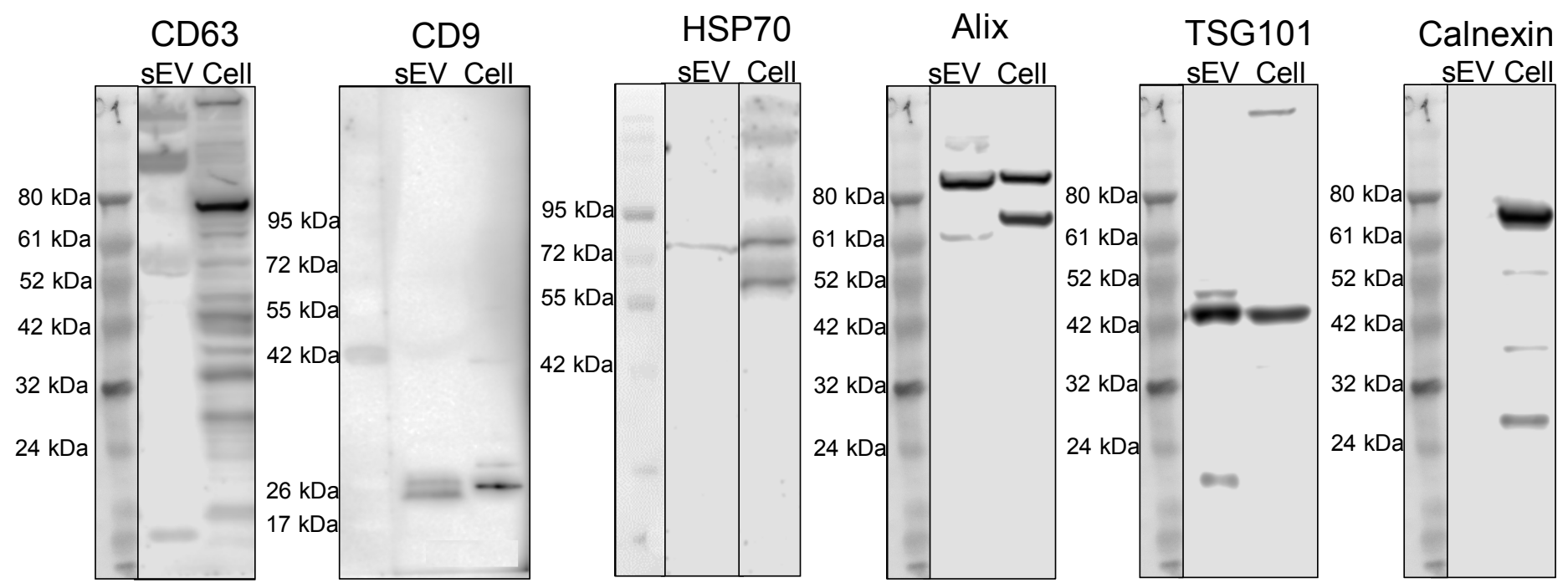

Supplementary Figure S1. Isolated B16F1 cell-derived vesicles showed exosomal characteristics. Spherical shape and small size of $s E V s$ were verified by AFM (top row). Size distribution of the isolated sEV population was analysed by DLS using a Zetasizer Nano S instrument (middle row). EV markers such as CD63 and CD9 (transmembrane proteins), HSP70, Alix and TSG101 (cytosolic proteins), Calnexin (negative sEV marker) were investigated in the vesicle isolates and the donor cell lysates by Western blot (bottom row). sEV and Cell samples were investigated on the same gels/blots for each marker, however their lines were cropped, shown by black boxes. 
DLS by Zetasizer Nano ZS ZEN 4003

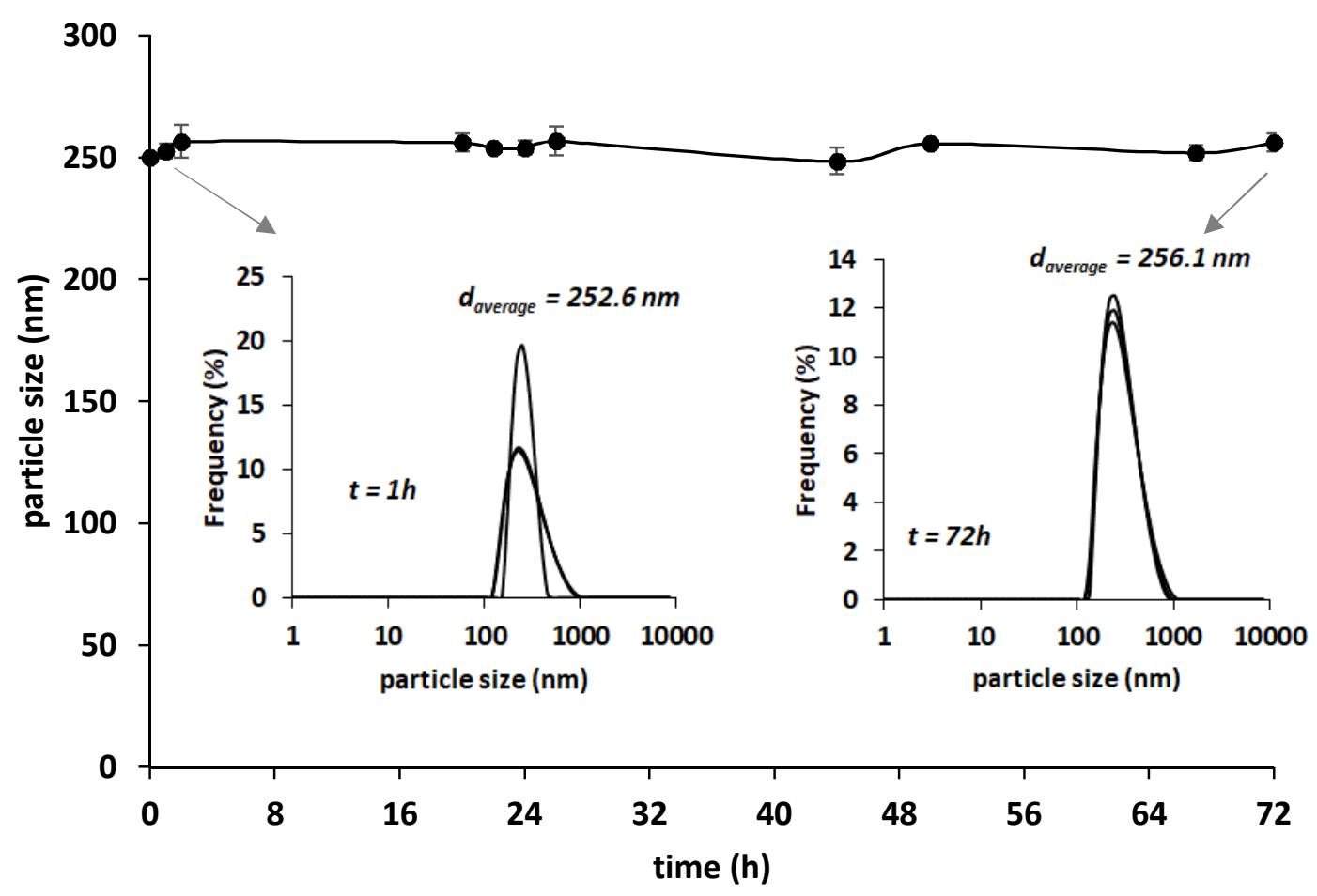

$\mathrm{CL}$ detection of $\mathrm{Ag}-\mathrm{TiO}_{2}$ in sEVs

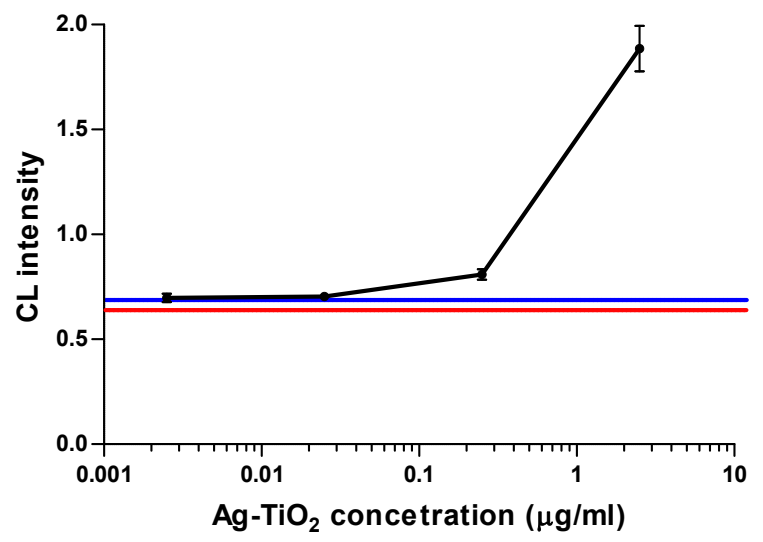

TEM of Ag-TiO ${ }_{2}$ sEVs

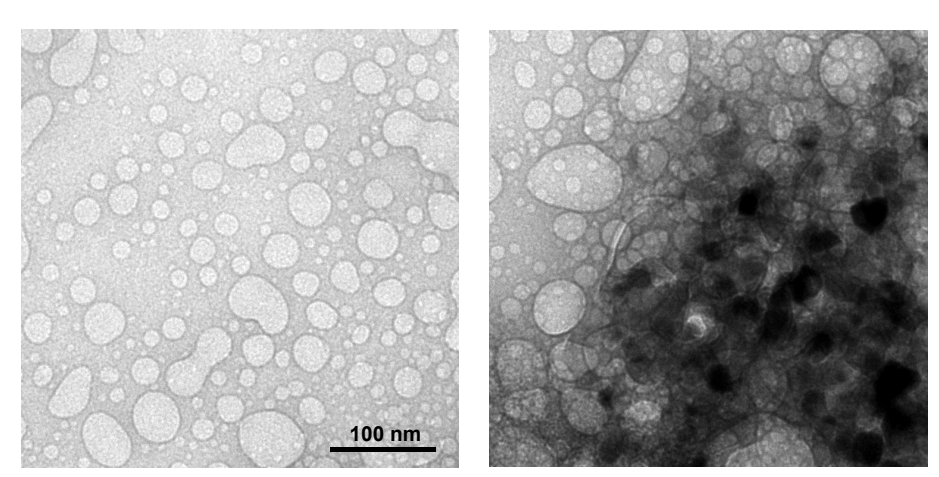

Supplementary Figure $\mathbf{S 2}$. Ag- $\mathrm{TiO}_{2}$ transfer by sEVs cannot be proven.

Upper diagram shows the size distribution of the $\mathrm{Ag}-\mathrm{TiO}_{2}$ photocatalyst particles, which was around $255 \mathrm{~nm}$ during the whole time interval of stress exposures. Measurements were performed by DLS, figure shows the measured average hydrodynamic particle diameter values of $\mathrm{Ag}-\mathrm{TiO}_{2}$ particles in B16F1 culture medium, as a function of time. The inserted figures show the size distribution curves measured at $1 \mathrm{~h}$ and $72 \mathrm{~h}$, respectively.

Lower diagram shows the result of the $\mathrm{Ag}-\mathrm{TiO}_{2}$ detection using chemiluminescence method. Black line is the $\mathrm{Ag}-\mathrm{TiO}_{2}$ calibration curve (each point represent mean $\pm \mathrm{SD}$ values). Blue and red lines show the mean $\mathrm{CL}$ intensities of the $\mathrm{Ag} \mathrm{Ctrl} \mathrm{sEV}$ (background) and $\mathrm{Ag}-\mathrm{TiO}_{2} \mathrm{sEV}$ lysates, respectively.

Left TEM image shows the $\mathrm{Ag}-\mathrm{TiO}_{2} \mathrm{sEVs}$, which do not contain inner structures, including electrondense $\mathrm{Ag}-\mathrm{TiO}_{2}$ nanoparticles. Right TEM image shows EVs mixed with $\mathrm{Ag}-\mathrm{TiO}_{2}$ nanoparticles as a reference. Cause of the high concentration of vesicles and nanoparticles, they are seen above each other. 


\section{Sequencing}

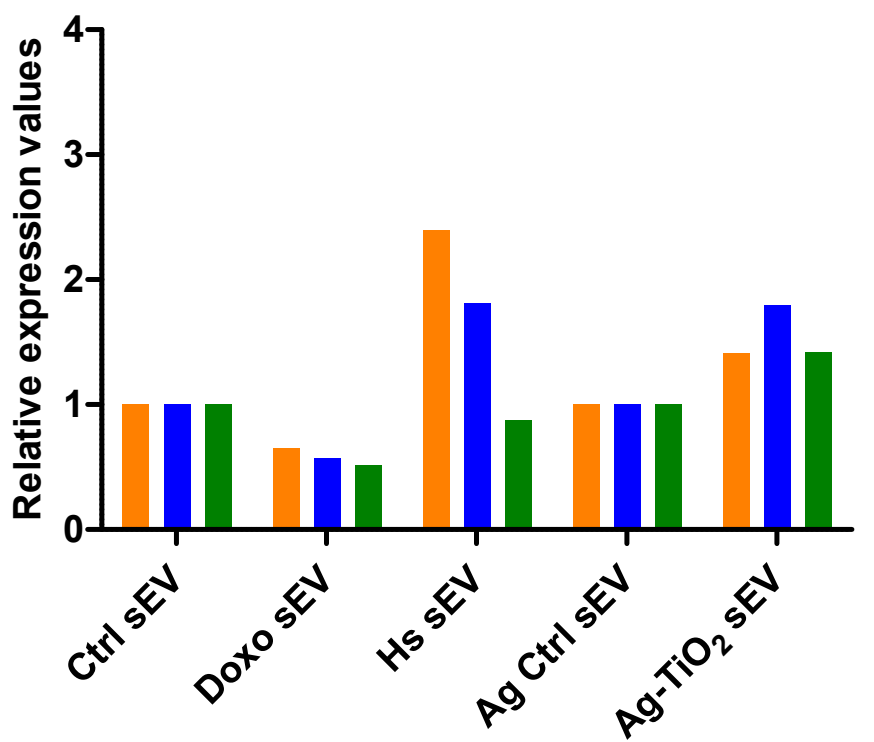

mmu-miR-16-5p

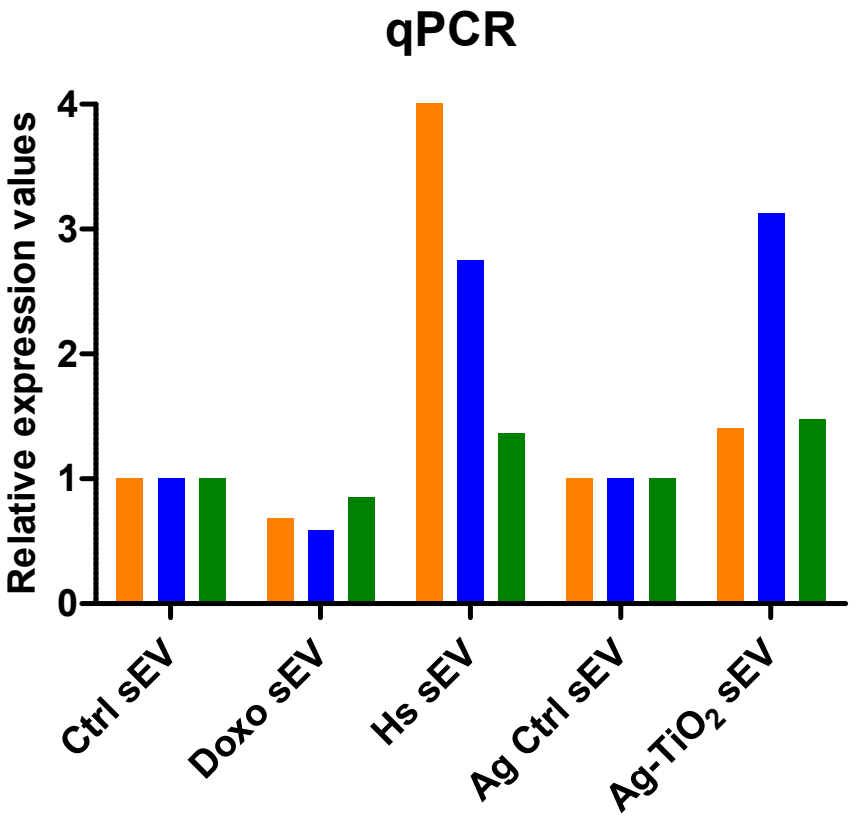

mmu-miR-29a-3p

Supplementary Figure S3. Validation of the miRNA sequencing results by qPCR. Relative expression values of mmu-miR-16-5p, mmu-miR-125b-5p and mmu-miR-29a-3p showed similar patterns for both quantification methods. 

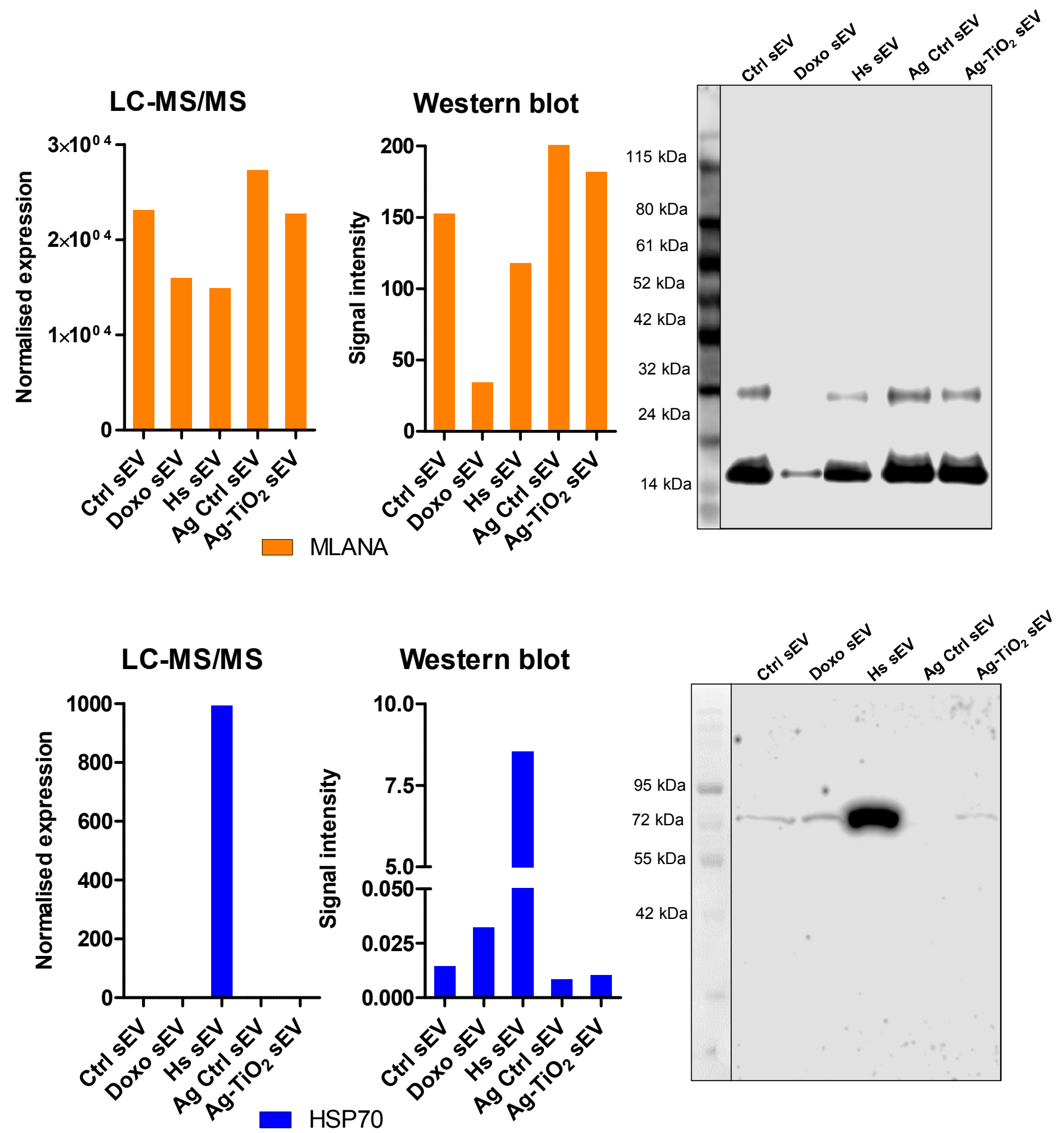

Supplementary Figure S4. Validation of the LC-MS/MS results by Western blot. Signal intensities of HSP70 and MLANA were quantified by densitometry, which reflected the LC-MS/MS resulted differences between the SEV groups. However, using Western blot, which is a more sensitive method, we were able to detect HSP70 in each SEV isolate. SEV and Cell samples were investigated on the same gels/blots for each marker, however their lines were cropped, shown by black boxes. 

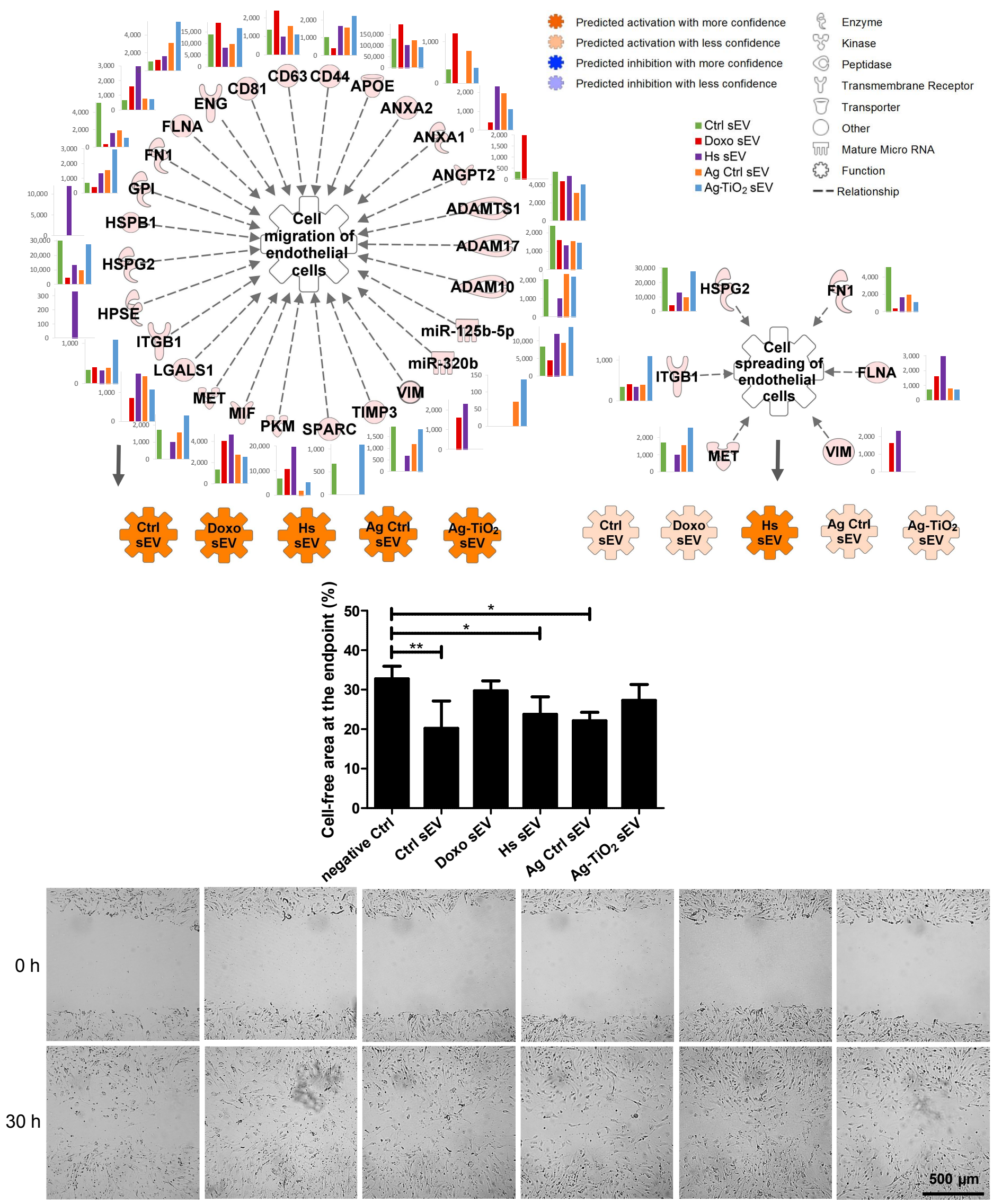

Supplementary Figure S5. Melanoma sEVs facilitate the endothelial cell migration. Networks show the IPA predictions for the regulatory effects of SEV molecules on the 'Cell migration of endothelial cells' and ,Cell spreading of endothelial cells'. Networks show every upstream regulator proteins and miRNAs accompanied by a bar graph, which represents the normalised expression values of the molecule for each $s E V$ group. Coloured symbols, named as the $s E V$ groups, display the expected regulation changes of the analysed 'Biofunctions' upon exposure to the vesicles. Bar graph shows the result of the analysis of wound closures by the ImageJ wound healing tool. It shows mean+SD values $(n=4)$. Images represent the wounds after $30 \mathrm{~h}$ of $\mathrm{sEV}$ exposures. 


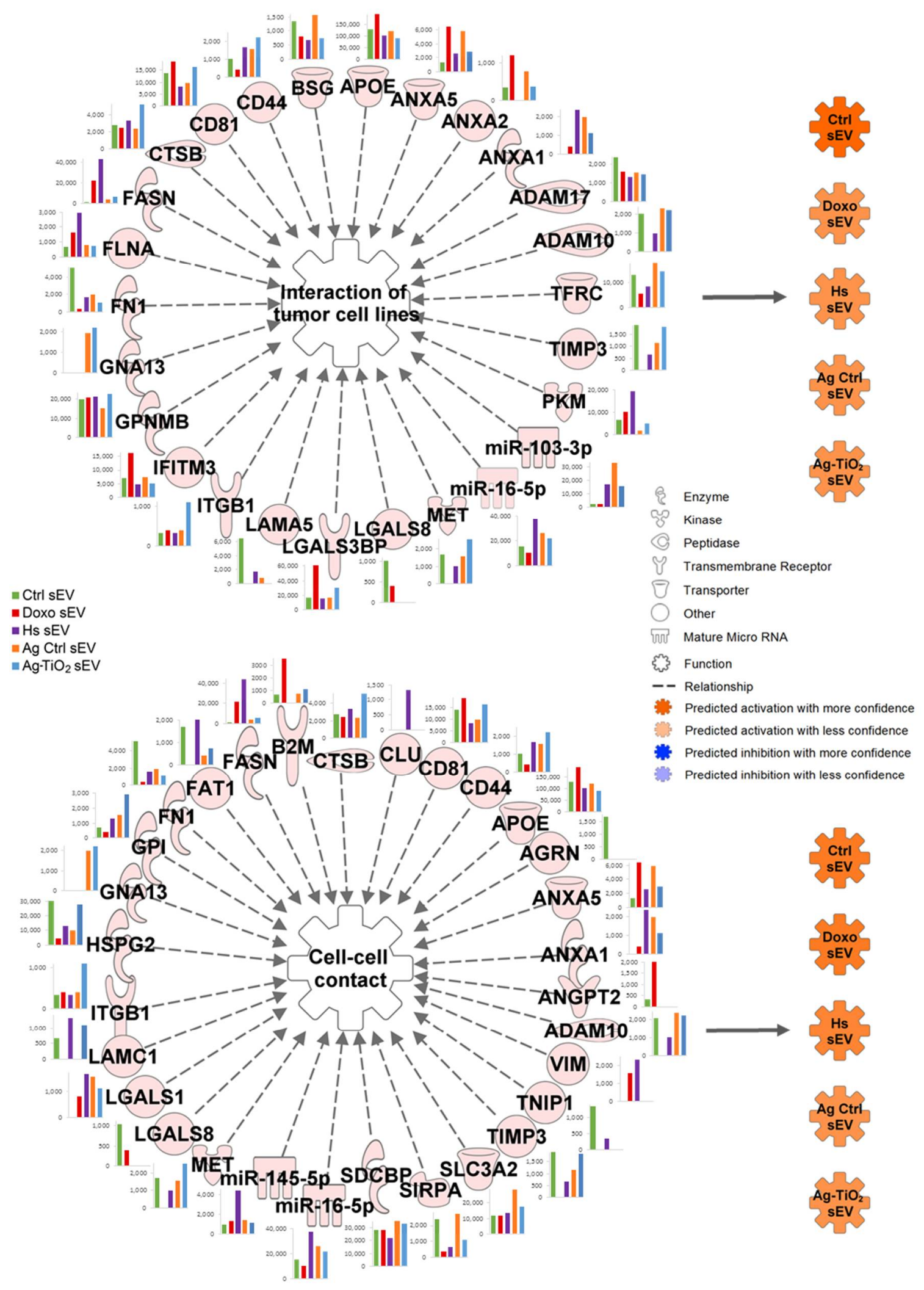

Supplementary Figure S6. IPA predictions for regulatory effects of sEV molecules on the 'Interaction of tumour cell lines' and the 'Cell-cell contact' showed activations. Networks show every upstream regulator proteins and miRNAs accompanied by a bar graph, which represents the normalised expression values of the molecule for each SEV group. Coloured symbols, named as the sEV groups, display the expected regulation changes of the analysed 'Biofunctions' upon exposure to the vesicles. 


\begin{tabular}{|l|c|c|c|c|c|c|}
\hline Group name & negative Ctrl & Ctrl sEV & Doxo sEV & Hs sEV & Ag Ctrl sEV & Ag-TiO $_{\mathbf{2}}$ sEV \\
\hline Eq. diameter $(\mu \mathrm{m})$ & $437 \pm 9$ & $351 \pm 15$ & $309 \pm 16$ & $423 \pm 5$ & $347 \pm 18$ & $348 \pm 17$ \\
\hline Major diameter $(\mu \mathrm{m})$ & $519 \pm 17$ & $489 \pm 20$ & $388 \pm 23$ & $530 \pm 20$ & $448 \pm 30$ & $432 \pm 32$ \\
\hline Minor diameter $(\mu \mathrm{m})$ & $323 \pm 12$ & $170 \pm 40$ & $242 \pm 17$ & $306 \pm 19$ & $212 \pm 20$ & $257 \pm 30$ \\
\hline Convexity & $0.662 \pm 0.037$ & $0.632 \pm 0.017$ & $0.730 \pm 0.064$ & $0.585 \pm 0.050$ & $0.690 \pm 0.165$ & $0.630 \pm 0.069$ \\
\hline Solidity & $0.774 \pm 0.021$ & $0.734 \pm 0.011$ & $0.838 \pm 0.009$ & $0.800 \pm 0.020$ & $0.825 \pm 0.080$ & $0.833 \pm 0.046$ \\
\hline Sphericity & $0.554 \pm 0.042$ & $0.501 \pm 0.013$ & $0.629 \pm 0.042$ & $0.491 \pm 0.034$ & $0.601 \pm 0.180$ & $0.547 \pm 0.066$ \\
\hline
\end{tabular}
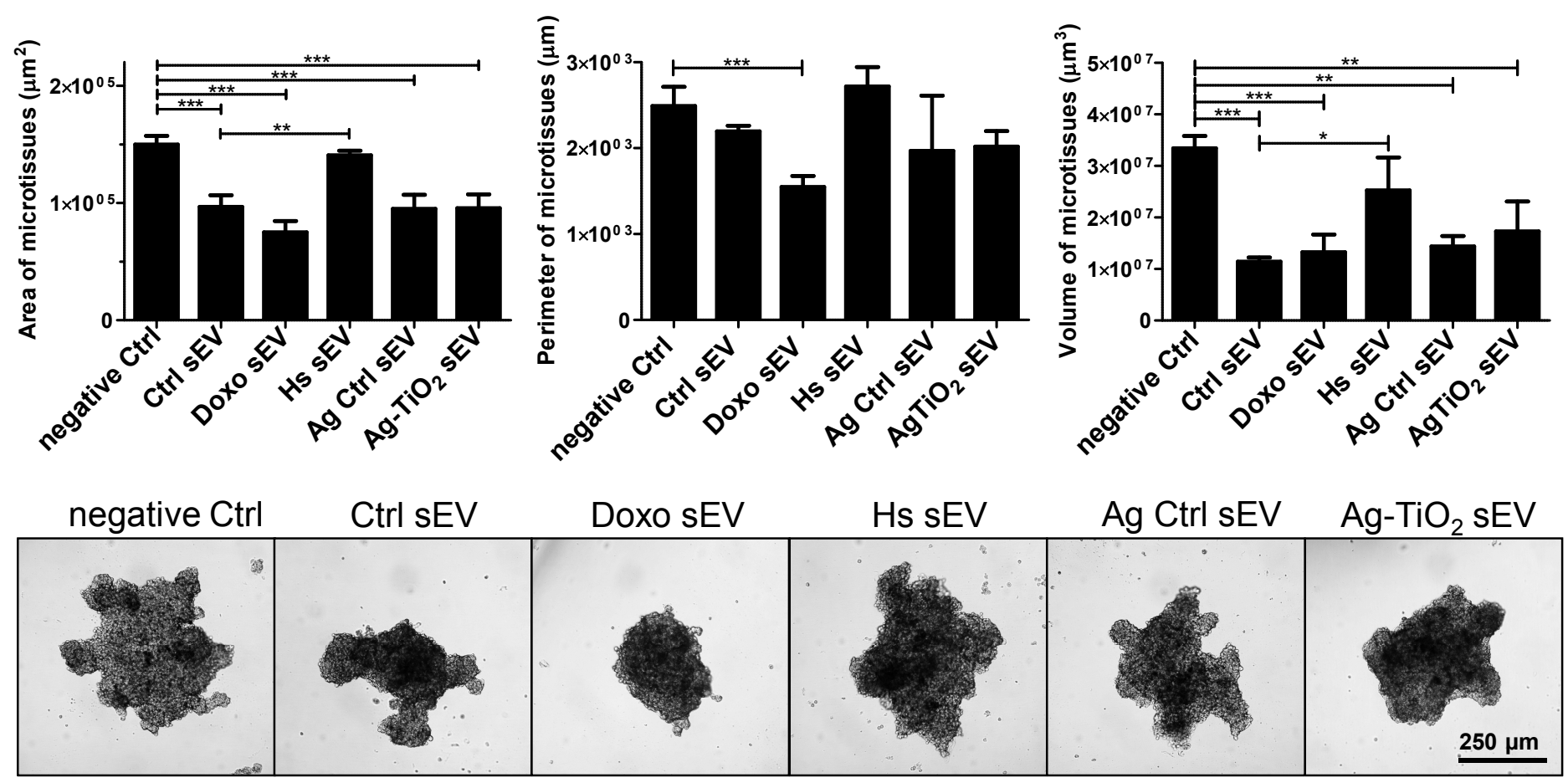

Supplementary Figure S7. Validation of the microtissue generation promoting effects of sEVs using MEFB16F1 3D co-cultures.

Table shows the statistics about the calculated diameters and compactness using the AnaSP software. Eq. diameter means equivalent diameter, major and minor diameters are measured through centroid. Table contains mean $\pm S D$ values.

Bar graphs show the area, perimeter and volume statistics of the generated microtissues (mean+SD, $n=3$ ). Statistical evaluation was performed by Welch's ANOVA test with Tukey's HSD post-hoc test; * $p<0.05$, ${ }^{* *} p<0.01$ and $* * * p<0.001$ indicate statistical significance.

Images represent the generated microtissues after $72 \mathrm{~h}$ of $s E V$ exposures. 
II. 


\title{
Stressors alter intercellular communication and exosome profile of nasopharyngeal carcinoma cells
}

\author{
Maria Harmati ${ }^{1}$, Zsofia Tarnai ${ }^{2}$, Gabor Decsi ${ }^{2}$, Sandor Kormondi ${ }^{3}$, Zsolt Szegletes ${ }^{4}$, Laszlo Janovak ${ }^{5}$, \\ Imre Dekany ${ }^{6}$, Okay Saydam ${ }^{7}$, Edina Gyukity-Sebestyen', Gabriella Dobra ${ }^{1}$, Istvan Nagy ${ }^{8}$, Katalin Nagy ${ }^{2}$, \\ Krisztina Buzas ${ }^{1,2}$ \\ ${ }^{1}$ Laboratory of Microscopic Image Analysis and Machine Learning, Institute of Biochemistry, Biological Research Centre of the \\ Hungarian Academy of Sciences, Szeged, Hungary; ${ }^{2}$ Department of Oral Biology and Experimental Dental Research, Faculty of Dentistry, \\ University of Szeged, Szeged, Hungary; ${ }^{3}$ Department of Traumatology, Faculty of Medicine, University of Szeged, Szeged, Hungary; \\ ${ }^{4}$ Biological Application of the Atomic Force Microscope Research Group, Institute of Biophysics, Biological Research Centre of the \\ Hungarian Academy of Sciences, Szeged, Hungary; ${ }^{5}$ Department of Physical Chemistry and Materials Sciences, University of Szeged, \\ Szeged, Hungary; ${ }^{6}$ MTA-SZTE Supramolecular and Nanostructured Materials Research Group, University of Szeged, Szeged, Hungary; \\ ${ }^{7}$ Molecular Neuro-Oncology Research Unit, Medical University of Vienna, Vienna, Austria; ${ }^{8}$ Sequencing Platform, Institute of \\ Biochemistry, Biological Research Centre of the Hungarian Academy of Sciences, Szeged, Hungary
}

BACKGROUND: Head and neck cancers comprise the sixth most common cancer type worldwide. One of the most remarkable malignancies of the head and neck is the cancer of the nasopharynx, with a strong metastatic tendency already in the early stage. Besides the conventional pathways of metastasis formation, the information content of exosomes produced by the cancer cells may play a key role in metastatic transformation. The aim of this study was to investigate how stressors alter the characteristic of tumor derived exosomes.

METHODS: In our experimental model, we compared the quantity and content of exosomes produced by a nasopharyngeal carcinoma cell line (5-8F) under conventional (chemotherapy) and alternative $\left(\mathrm{Ag}-\mathrm{TiO}_{2}-\right.$ catalyzed reactive oxygen species generation) cytostatic treatment. After isolation, exosomes were identified by atomic force microscopy and quantified with Nanosight NS500 device. MicroRNA content of them was analyzed using SOLiD 5500xl technology. The sequences were annotated in CLC Genomics Workbench version 5.5.I.

RESULTS: Beyond the classic chemotherapeutic agent (doxorubicin), $\mathbf{A g}-\mathrm{TiO}_{2}$ in a photo-catalytic process also showed cytostatic activity. Tumor cell damage induced by the cytostatic treatments significantly altered the number of released exosomes and led to the predominance of tumor suppressors in the exosomal miRNA profile.

CONCLUSIONS: Our results suggest that the intercellular communication between tumor cells and surrounding stroma cells can be altered by microenvironment

Correspondence: Krisztina Buzas, Hungarian Academy of Sciences, Biological Research Center, Temesvári krt. 62., Szeged H-6726, Hungary. Tel: +3662599600, Fax: +3662433506, E-mail: kr.buzas@gmail.com Accepted for publication July 19, 2016 which increased quantity of exosomes and diversity of miRNAs in this study. Imbalance of oncogenic and tumor suppressor miRNAs caused by cytostatic treatments may influence the antiproliferative and metastasis inhibitory effect of cytostatic agents.

J Oral Pathol Med (2016)

Keywords: cytostatic agents; exosomes; microRNAs; nanoparticles; nasopharyngeal carcinoma

\section{Introduction}

Exosomes and microvesicles (MVs) are nanometer-sized membrane vesicles secreted from various cell types (including tumor cells) into the extracellular milieu and body fluids. MVs and exosomes contain different kinds of biomolecules, such as proteins, lipids, and nucleic acids, whereby EVs are suggested to modulate the immune function, angiogenesis, cell proliferation, cell-to-cell communication, and tumor invasion $(1,2)$.

An increasing number of publications highlight the role of exosomes and microRNA carried by extracellular vesicles in tumor progression, metastatic activity, and also in tumor response under suboptimal conditions, for example, chemotherapy-induced cell damage $(3,4)$.

Head and neck cancers are a heterogeneous group of epithelial cancers (including the tumors of the larynx, hypopharynx, oropharynx, nasopharynx, the paranasal sinuses, and the oral and nasal cavity) that evolve from the oral and pharyngeal squamous epithelium.

Head and neck squamous cell carcinoma with an incidence of more than 600000 per year is the sixth most common cancer worldwide (5). The five-year survival rate 
for these patients is $50-60 \%$, which has not decreased in the last thirty years (6).

Nasopharyngeal carcinoma (NPC) is a distinct cancer type of the head and neck region, differing from other cancers in terms of its epidemiology, etiology, clinical behavior, and response to treatment.

The prognosis of NPC depends on the metastatic activity and loco-regional spread (7). Unfortunately, NPC has an early metastatic tendency (8). The metastatic potential is considerably high: $60-90 \%$ of the patients develop metastases in the regional lymphatic nodes (9).

In the background of this high metastatic activity may stand an additional mechanism of metastasis formation mediated by the exosomes produced by cancer cells (1).

NPC-derived exosomes contain a variety of bioactive molecules, such as proteins, lipids, and nucleic acids like microRNAs (miRNAs). The latter are small, non-coding post-transcriptional regulators which are considered to play a key role in biological processes and tumor development. MiRNAs have been found to regulate many kinds of genes including development, proliferation, differentiation, and stress response (10).

MicroRNAs might also be good biomarkers for cancer detection, as miRNAs are remarkably stable in blood and it seems that each malignant disease has its own, specific miRNA expression profile (11). Being able to offer a reliable prognosis of NPC by miRNA characteristics would be a significant improvement in the battle against the disease.

The purpose of this study was to investigate how exosomal miRNAs might assist metastasis formation in NPC and how cytostatic therapies as stressors influence the characteristics of the tumor-derived exosomes.

In our experimental model, we examined the exosomeproducing capacity of an NPC cell line under different cytostatic treatments. The miRNA content of the exosomes was also examined. We sought to answer whether cytostatic therapy could alter the quantity and contents of the tumorderived exosomes. 5-8F NPC cells were exposed to two different types of cytostatic treatment, namely the classical chemotherapy with doxorubicin and a new method for the inhibition of cell proliferation, in which we utilized the photocatalytic activity of $\mathrm{Ag}-\mathrm{TiO}_{2}$. The effects of these treatments were compared in terms of exosome output and miRNA profile.

Doxorubicin - the antracycline antibiotic widely used in the chemotherapy of several cancer types-exerts its cytostatic effect by intercalating the DNA.

$\mathrm{Ag}-\mathrm{TiO}_{2}$ photocatalyst particles irradiated with exciting wavelength light show photocatalytic activity. Photocatalysis is a photo-induced reaction, in which the photons are exiting the photocatalyst particles. During the photocatalytic process, the irradiated photocatalyst particles produce highly reactive oxygen species such as superoxide $\left(\mathrm{O}_{2}{ }^{-}\right)$, hydrogen peroxide $\left(\mathrm{H}_{2} \mathrm{O}_{2}\right)$, or hydroxyl radical ( $\left.\mathrm{HO}\right)(12)$. Due to these reactive radicals, the photocatalyst particles can degrade many organic compounds and inactivate microorganisms via destroying the cell wall and the DNA $(12,13)$. However, $\mathrm{TiO}_{2}$ needs high-energy (UV) photons to be activated, and has a low quantum efficiency due to the $\mathrm{TiO}_{2}$ wide band gap $\left(E_{g}=3.2 \mathrm{eV}\right.$ for anatase) and fast recombination rate of photo-generated electron and hole pairs.
Silver nanoparticles-functionalized $\mathrm{TiO}_{2}$ photocatalysts $\left(\mathrm{Ag}-\mathrm{TiO}_{2}\right)$ enhance the light absorbance of the catalyst in the visible range and significantly improve the photocatalytic activity of the metal oxide semiconductor under UVvisible light (14).

We found that tumor cell damage induced by the cytostatic treatments significantly increased the quantity of the exosomes and altered their miRNA content. This significantly increased quantity of the exosomes may potentiate the information transfer of tumor cells to the surrounding stroma cells and affect the mechanisms of metastasis formation.

\section{Materials and methods}

\section{Synthesis of $\mathrm{Ag}-\mathrm{TiO}_{2}$ photocatalyst particles}

Commercially available $\mathrm{TiO}_{2}$ (Degussa P25 from Evonik Gmbh) photocatalyst with a specific surface area of $\sim 50 \mathrm{~m}^{2} /$ $\mathrm{g}$ was used as a standard photocatalyst without any treatment. Plasmonic Ag nanoparticles were prepared on the surface of $\mathrm{TiO}_{2}$ to enhance the photocatalytic efficiency of the prepared $\mathrm{Ag}-\mathrm{TiO}_{2}$ sample. The prepared $\mathrm{Ag}-\mathrm{TiO} \mathrm{O}_{2}$ photocatalyst contained 0.5 wt. $\%$ surface silver nanoparticles. The detailed process of the $\mathrm{Ag}-\mathrm{TiO}_{2}$ nanoparticle synthesis was published in our earlier publications $(13,14)$.

\section{Cell line and culture conditions}

5-8F human NPC cell line was kindly provided by Ji Ming Wang (NCI-Frederick, MD, USA). Cell cultures were stored at $37^{\circ} \mathrm{C}$ in a humidified incubator with $5 \% \quad \mathrm{CO}_{2}$, in Dulbecco's modified Eagle's medium (DMEM) supplemented with $10 \%$ fetal bovine sera depleted of contaminating vesicles, $1 \%$ MEM non-essential amino acids, $1 \%$ MEM vitamin solution, and $100 \mathrm{U} / \mathrm{ml}$ penicillin-streptomycin (all from Lonza, Basel, Switzerland).

There was no ethical approval required for this study.

\section{Cell culture treatments and exosome isolation}

To investigate the changes of exosome production under suboptimal conditions, cell cultures were treated at $90 \%$ confluency in three different ways: The medium was replaced with (i) fresh media, (ii) fresh media supplemented by $0.6 \mu \mathrm{M}$ doxorubicin, and (iii) fresh media supplemented by lightinduced $2.5 \mu \mathrm{g} / \mathrm{ml} \mathrm{Ag}-\mathrm{TiO}_{2}$ aqueous nanodispersion (this concentration was chosen based on the in vitro tests as well as limitation of in vivo application). The photoreactive $\mathrm{Ag}-\mathrm{TiO}_{2}$ nanoparticles were induced by a low-pressure mercury lamp (GCL303T5/4 type, LightTech, Dunakeszi, Hungary) mostly emitting light wavelength $\lambda \geq 360 \mathrm{~nm}$, for 10 min from a 10 $\mathrm{cm}$ distance in one-third medium volume. After the light induction, the medium was supplemented to the total volume. This step was necessary because of the light absorption and reflection of the medium layer in the dishes which would otherwise prevent the induction of $\mathrm{Ag}-\mathrm{TiO}_{2}$. After $72 \mathrm{~h}$, supernatants were collected and the exosomes were isolated by differential filtration and ultracentrifugation as described previously (15).

\section{Cell viability assay}

To characterize the effects of suboptimal conditions regarding viability, the cells were treated with different 
concentrations of doxorubicin and light-induced photoreactive $\mathrm{Ag}-\mathrm{TiO}_{2}$ dispersion. After $72 \mathrm{~h}$, cell viability was determined by trypan blue exclusion.

\section{Identification of exosomes}

Atomic force microscopic (AFM) measurements were carried out with an Asylum MFP-3D head and controller (Asylum Research, Santa Barbara, CA, USA). The driver program MFP-3D Xop was written in IGOR Pro Software (Wavemetrics, Lake Oswego, OR, USA). For imaging, gold-coated silicon nitride rectangular cantilevers were used with a typical spring constant of $0.03 \mathrm{~N} / \mathrm{m}$ in air (BLRC150 VB, Olympus Optical Co. Ltd., Tokyo, Japan). The spring constant for each cantilever was determined by
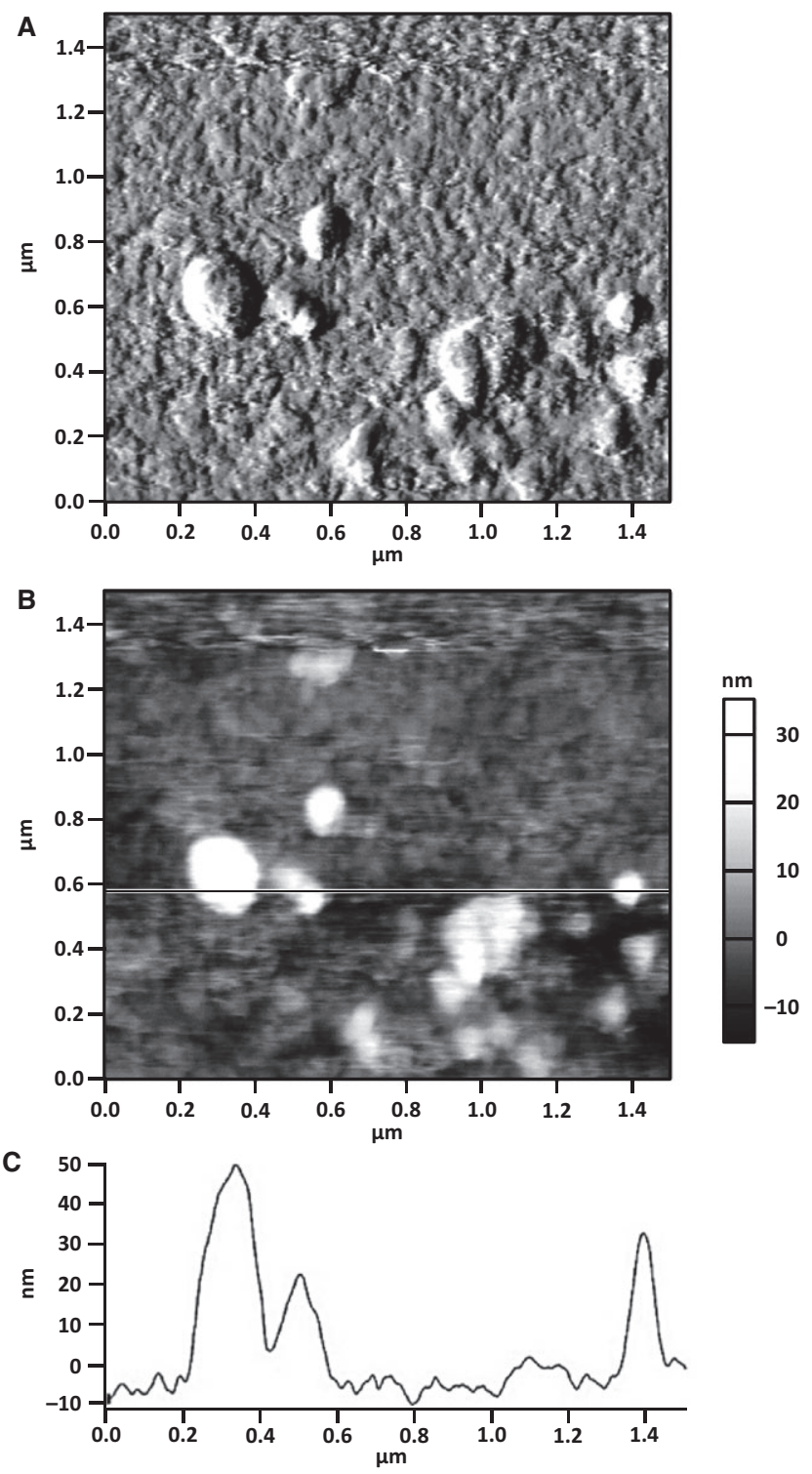

Figure 1 High-resolution AFM images of intact exosomes. Height (A) and amplitude $(\mathrm{B})$ images $\left(1.5 \times 1.5 \mathrm{~mm}^{2}\right)$ are shown together with height profiles (C) corresponding to the horizontal line assigned on the height images. Exosomes were attached to the APTES-covered mica surface with glutaraldehyde. thermal calibration followed by Sader's method (16-19). For the measurements, freshly cleaved $1 \times 1 \mathrm{~cm} \mathrm{mica}$ (SPI-Chem Mica Sheets) surfaces were used as supports for exosomes and microvesicles. The negatively charged mica surfaces were incubated in 2\% APTES ((3-aminopropyl) triethoxysilan, Sigma, St. Louis, MO, USA) dissolved in isopropanol for $90 \mathrm{~min}$ in shaking condition at RT to create free amine groups on their surface $(18,20,21)$. Exosomes and microvesicles were attached to the modified surface with glutaraldehyde. Measurements were carried out in tapping (AC) mode in PBS solution. Typically, $512 \times 512$ point scans were taken at $0.4 \mathrm{~Hz}$ scan rate. Both the trace and retrace images were recorded and compared. The measurements presented here are $1.5 \times 1.5 \mu \mathrm{m}^{2}$ flattened heights. Experiments were repeated three to five times.

\section{Measurement of the number distribution of exosomes}

Nanoparticle tracking analysis (NTA) was used to compare the number and size of the exosomes isolated from the three different supernatants. NTA is the commercial name of an optical particle tracking which is an accepted method for obtaining concentration and size distribution of EV populations, including exosomes (22). These measurements were performed by the NanoSight's NS500 device.
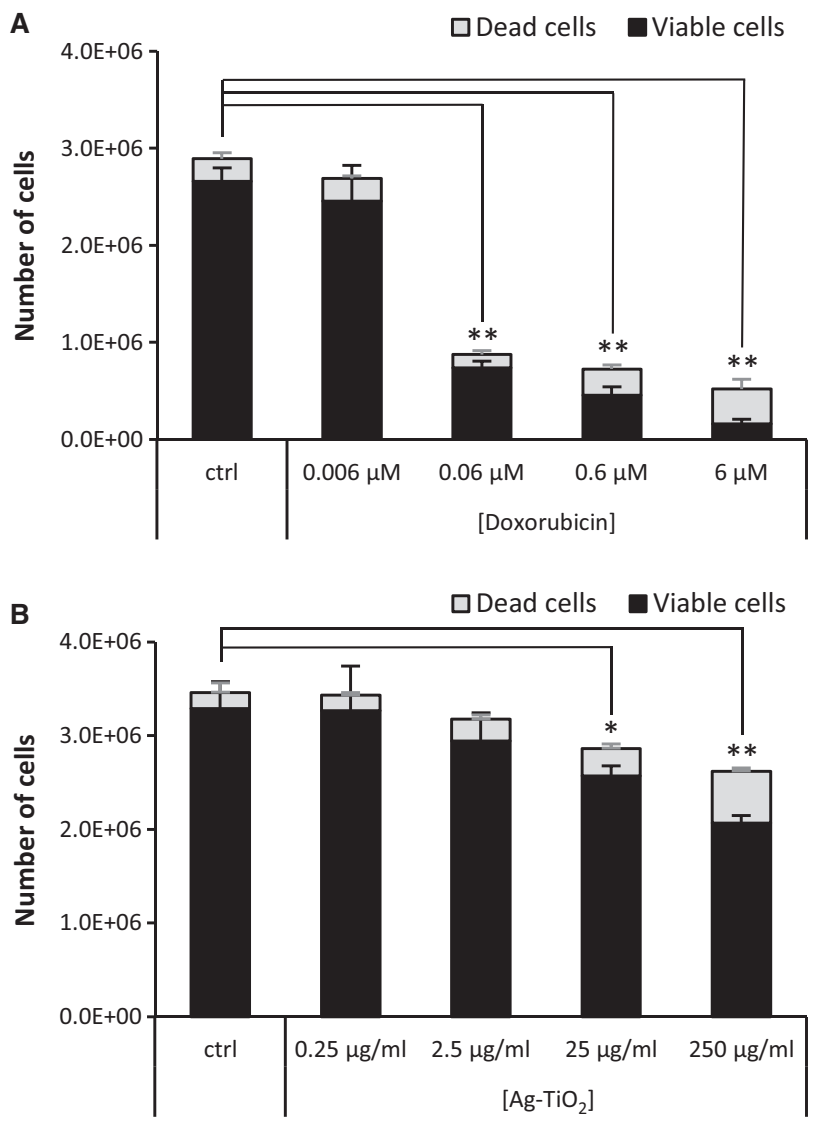

Figure 2 Cytostatic and antiproliferative effects of doxorubicin and $\mathrm{Ag}-\mathrm{TiO}_{2}$ treatments on 5-8F NPC cell cultures. Number of viable cells significantly decreased $72 \mathrm{~h}$ after (A) $0.06-6 \mu \mathrm{M}$ doxorubicin (**P<0.001) and (B) $25-250 \mu \mathrm{g} / \mathrm{ml} \mathrm{Ag-TiO}{ }_{2}$ treatments $(25 \mu \mathrm{g} / \mathrm{ml} \mathrm{Ag}-$ $\left.\mathrm{TiO}_{2}: * P=0.003 ; 250 \mu \mathrm{g} / \mathrm{ml} \mathrm{Ag}-\mathrm{TiO}_{2}: * * P<0.001\right)$. 


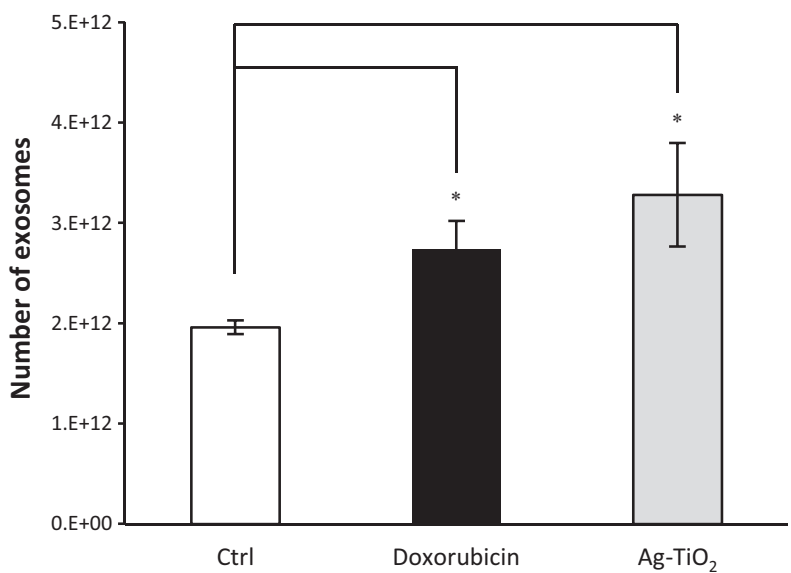

Figure $35-8 \mathrm{~F}$ human NPC cell-derived exosome production under suboptimal conditions. $0.6 \mu \mathrm{M}$ doxorubicin and $2.5 \mu \mathrm{g} / \mathrm{ml} \mathrm{Ag}-\mathrm{TiO}_{2}$ treatments significantly increased the exosome production in number $\left({ }^{*} P<0.001\right)$

\section{MiRNA analysis of exosomes}

In order to determine differences of the miRNA content of exosomes derived from the control, doxorubicin- or Ag$\mathrm{TiO}_{2}$-treated cell culture supernatants, six parallel but independent experiments were performed to collect supernatant and isolate exosomes. MiRNA was isolated from all samples by the NucleoSpin miRNA kit (Macherey-Nagel, Düren, Germany) according to the manufacturer's instructions and analyzed using the SOLiD 5500xl technology. The sequences were annotated in CLC Genomics Workbench version 5.5.1 as described previously (23).

\section{Statistical analysis}

Data analyses were performed using two-tailed Student's $t$ test by Microsoft Excel, and $P \leq 0.05$ was regarded as statistically significant.

\section{Results}

Exosome identification

In situ AFM measurements were used to characterize the size and shape distribution of exosomes. This method is suitable to define the size and mechanical properties of the particles; therefore, the exosomes can be distinguished from viruses or other contaminations. We have found the appropriate shape and size of exosomes in $30-70 \mathrm{~nm}$ (Fig. 1).

Cell viability changes under suboptimal conditions

To characterize the effects of suboptimal conditions for the viability of cells, we treated the cell cultures with doxorubicin or light-induced $\mathrm{Ag}-\mathrm{TiO}_{2}$. Our results verified the already established concentration-dependent cytotoxicity

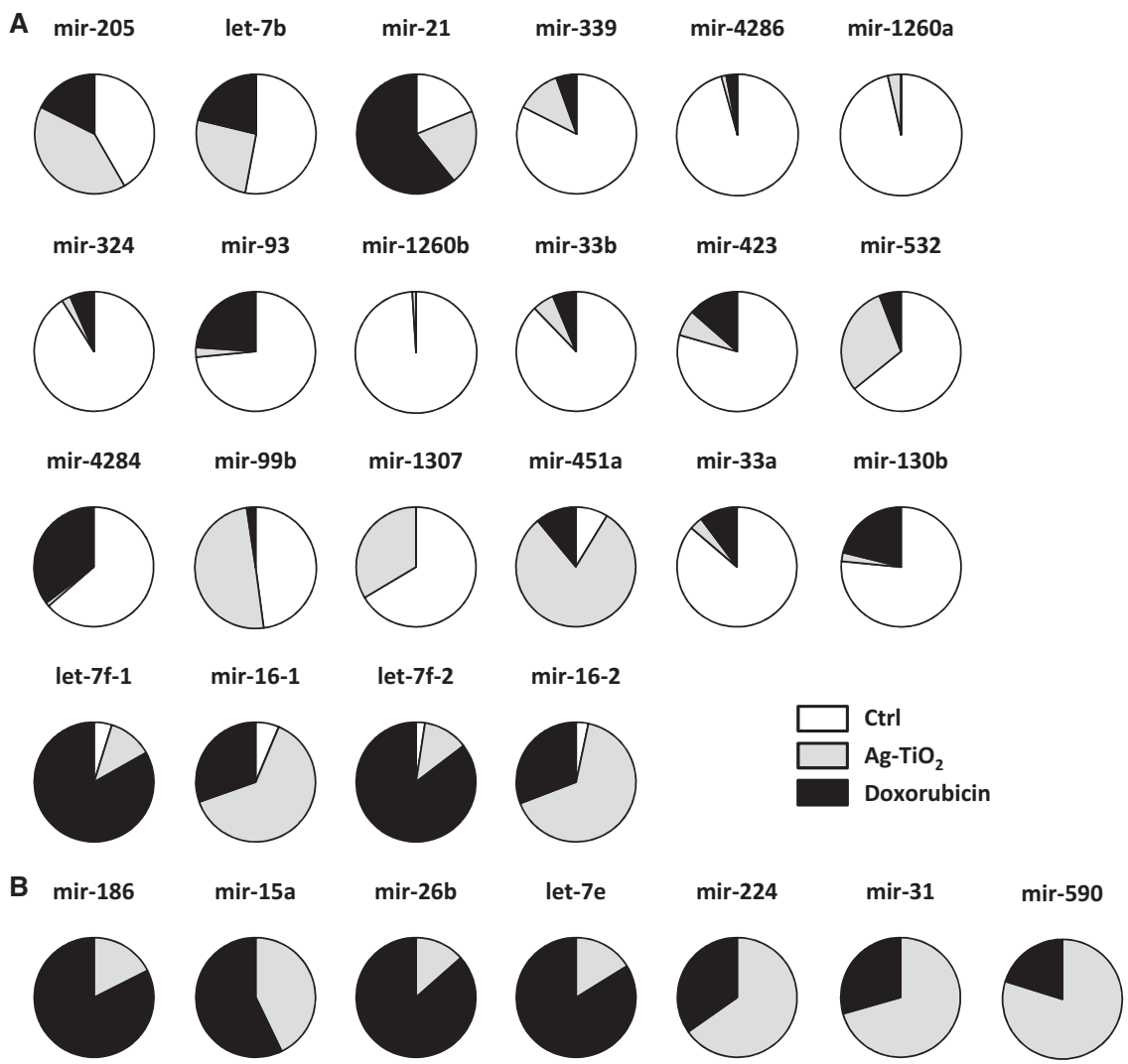

Figure 4 Ratio of miRNAs carried by the different exosomes. (A) miRNAs that markedly (tenfold increase or 0.1-fold decrease) changed during cell culturing under cytostatic treatment. (B) The most abundant miRNAs that were exclusively detected in one or both group of the treated 5-8F NPC cellderived exosomes. 


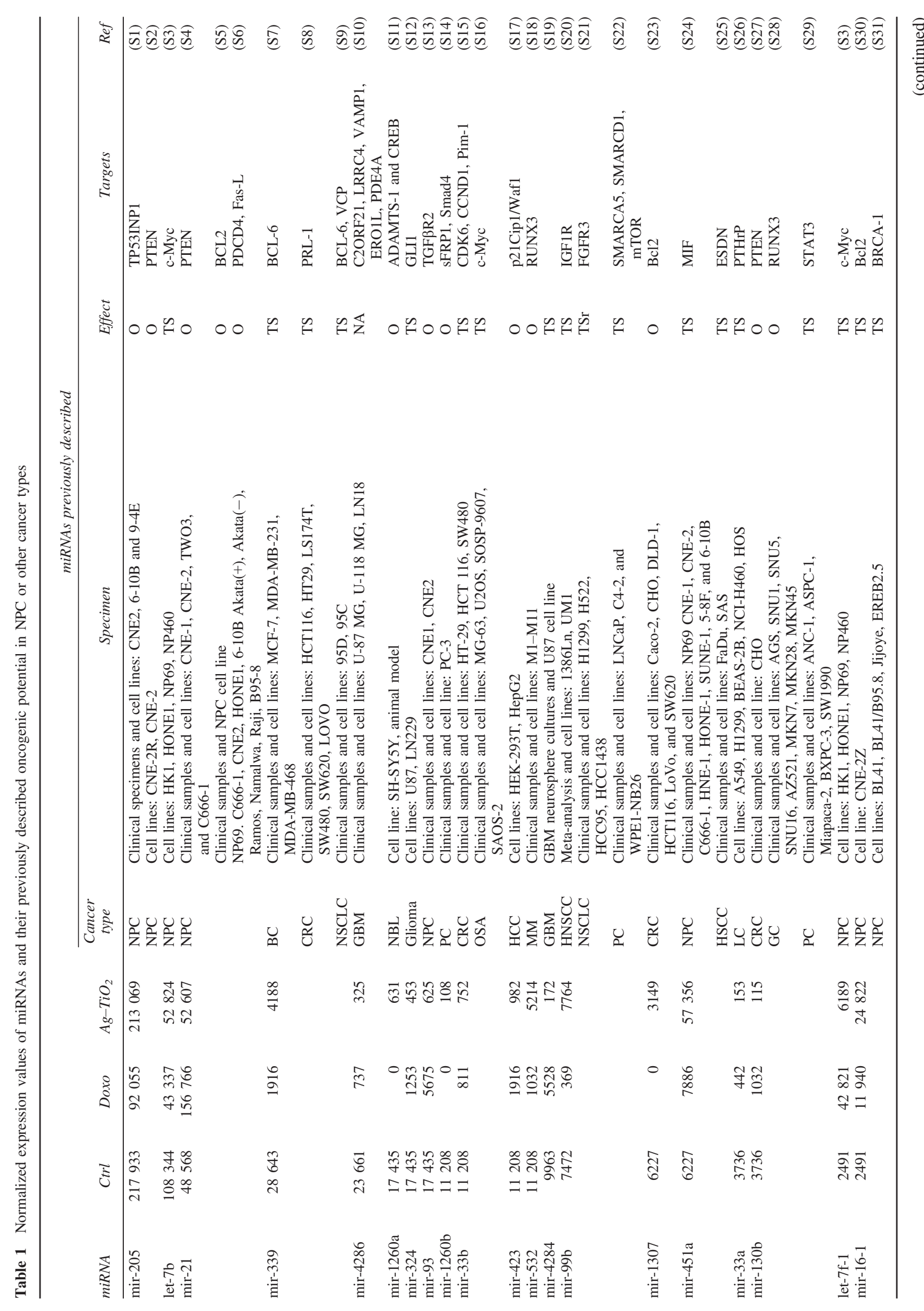


and antiproliferative effect of doxorubicin (Fig. 2). A new observation was that photogenerated reactive free radicals produced by the $\mathrm{Ag}-\mathrm{TiO}_{2}$ photocatalyst particles as a result of photocatalysis reduced cell proliferation. Number of viable cells significantly decreased after treatment with $25 \mu \mathrm{g} / \mathrm{ml}(P=0.003)$ and $250 \mu \mathrm{g} / \mathrm{ml}(P<0.001)$ $\mathrm{Ag}-\mathrm{TiO}_{2}$.

\section{Distribution of exosomes}

Changes of the size and number distribution of exosomes derived from the differently treated cell cultures were determined by nanoparticle tracking analysis (NTA). We found that the size distribution did not significantly change after either doxorubicin or $\mathrm{Ag}-\mathrm{TiO}_{2}$ treatments (data not shown). In contrast, the quantity of produced exosomes exhibited a significant change under suboptimal conditions. $\mathrm{Ag}-\mathrm{TiO}_{2}$ treatment resulted in a remarkably increased exosome production $(P=0.0023)$ after $72 \mathrm{~h}$ of treatment. Similarly, the DNA damage induced by doxorubicin resulted in significantly $(P=0.001)$ elevated exosome production (Fig. 3).

\section{Altered exosomal miRNA content}

The qualitative analysis of exosome content targeted the miRNAs carried by the NPC-derived exosomes. The SOLiD $5500 \mathrm{xl}$ technology and the bioinformatics supported the high-quality screening of miRNA content.

MicroRNA diversity impressively increased under suboptimal conditions. In the control samples, 71 different types of miRNA were identified. This increased to 121 types in the doxorubicin-treated samples and to 223 in the $\mathrm{Ag}-\mathrm{TiO}_{2}$-treated ones.

Fourteen types of exosomal miRNA showed more than tenfold decrease after either doxorubicin or $\mathrm{Ag}-\mathrm{TiO}_{2}$ treatment (Fig. 4). More than half of these have oncogenic potential in NPC or other cancer types (Table 1). Although some of them (mir-33a, the let-7 family) have tumor suppressor functions, their role in the chemoresistance is also proven (24).

Fifty-seven and 152 miRNAs were detected exclusively in the doxorubicin-treated cell-derived exosomes and in the $\mathrm{Ag}-\mathrm{TiO}_{2}$-treated cell-derived exosomes, respectively (Fig. 4). Let-7e, mir-15a, mir-186, mir-26b, mir-224, mir31 , and mir-590 were the most abundant miRNA types among them. In addition, five other miRNA types (let-7f-2, let-7f-1, mir-16-2, mir-16-1, and mir-451a) showed tenfold or higher increase in one or both types of stress induced exosomes (Fig. 4). Most of these were previously described as tumor suppressors in NPC (Table 1).

Based on data from the literature (summarized in Table 1) about potential functions of listed miRNAs, NPC cells release tumor suppressor miRNA types via exosomes and retain the oncogenic and resistance-enhancing ones under stress to maintain their tumorigenecity. In contrast, under normal conditions, cells produce and release miRNA types that promote progression and metastasis formation in vivo.

The various types of miRNA can have different targets and functions depending on the tumor type, clinical stage, therapy, or other specific regulatory factors and conditions. For example, mir-130b has oncogenic functions in 
colorectal and gastric cancer (S27, S28), but in pancreatic cancer, its tumor suppressor role was proven (S29).

\section{Discussion}

Exosomes are the smallest class of extracellular vesicles by size, secreted via an evolutionally conserved, endosomal pathway. The content and function of exosomes are variable, defined by the structure of the mother cells and the microenvironment (25). In the present study, we characterized the 5-8F NPC cell line-derived exosomes by size and by microRNA content as the potent information packages of intercellular communication. While the size and shape distribution of the nanovesicles turned out to be unchanged by treatment, we observed significant changes in the quantity and quality of exosomes under suboptimal conditions.

Doxorubicin is a well-known anthracycline chemotherapeutic agent. Its therapeutic effect can be traced back to its ability to intercalate adjacent DNA base pairs, the inhibition of topoisomerase II, and the production of hydroxyl free radicals (26). Doxorubicin can also induce histone eviction from open chromatin (27). These effects lead to the inhibition of cell proliferation and apoptosis initiation in the rapidly replicating cells. However, we could detect significantly increased exosome production parallel with reduced cell viability. The DNA stress changed not only the number of secreted exosomes but induced marked changes in the microRNA profile.

Reactive oxygen species (ROS) generation by $\mathrm{Ag}-\mathrm{TiO}_{2}$ photocatalysis is a known phenomenon (13), and ROSinduced cell damage is not a new phenomenon either (28). However, their effects on exosome production have not been examined so far. Similar to doxorubicin exposure, the cell damage caused by $\mathrm{Ag}-\mathrm{TiO}_{2}$ photocatalysis-related ROS generation resulted in significantly increased exosome production and markedly altered miRNA profile.

Increased exosome production of stress exposed tumor cells has been observed in several studies (29-32). Kucharzewska and Belting conceptualized that different stress factors cause cellular accumulation of ceramide, which triggers increased production and secretion of exosomes as an adaptation to microenvironmental stress (33).

As for the miRNA profiles, the quantitative change of a specific miRNA type was defined as marked when the expression level of that specific type exhibited a tenfold change compared to its original (control) level. Marked change of 30 different miRNA types was observed. The tumor suppressor-like to oncogenic-like ratio was 20:10. Based on our observations, both stress conditions, $\mathrm{Ag}-\mathrm{TiO}_{2}$ and doxorubicin, also elevated exosome responses.

Although alterations of exosomal characteristics had similar trends under $\mathrm{Ag}-\mathrm{TiO}_{2}$ and doxorubicin stress, there are several miRNAs, such as mir-1260, mir-93, or mir-16-1 that showed different intensity or opposite changes compared to control miRNA levels. A plausible explanation for this, that the tested cytostatic agents have distinct mode of action in the cells resulting in different miRNA regulatory effects. Our data suggest that these stressors, besides their antiproliferative effect, may influence the intercellular communication of tumor cells. Increases in exosome release and imbalance of oncogenic and tumor suppressor exosomal miRNAs could have an additional effect on tumor development. For instance, the process of metastasis formation that depends on the microenvironment of the tumor cells $(34,35)$ can be influenced by doxorubicin or $\mathrm{Ag}-\mathrm{TiO}_{2}$ treatments, as tumor-derived exosomes internalized by stromal cells have an altered information content.

\section{References}

1. Kahlert C, Kalluri R. Exosomes in tumor microenvironment influence cancer progression and metastasis. J Mol Med (Berl) 2013; 91: 431-7.

2. Muralidharan-Chari V, Clancy JW, Sedgwick A, D'Souzaschorley C. Microvesicles: mediators of extracellular communication during cancer progression. J Cell Sci 2010; 123: $1603-11$.

3. Salido-Guadarrama I, Romero-Cordoba S, Peralta-Zaragoza O, Hidlago-Miranda A, Rodrigez-Dorantes M. MicroRNAs transported by exosomes in body fluids as mediators of intercellular communication in cancer. Onco Targets Ther 2014; 7: 132738.

4. Hayes J, Peruzzi PP, Lawler S. MicroRNAs in cancer: biomarkers, functions and therapy. Trends Mol Med 2014; 20: $460-9$.

5. Leemans CR, Braakhuis BJM, Brakenhoff RH. The molecular biology of head and neck cancer. Nat Rev Cancer 2011; 11: 922.

6. Pulte D, Brenner H. Changes in survival in head and neck cancers in the late 20th and early 21st century: a period analysis. Oncologist 2010; 15: 994-1001.

7. Arango BA, Castrellon AB, Perez CA, et al. Nasopharyngeal carcinoma: alternative treatment options after disease progression. Expert Rev Anticancer Ther 2010; 10: 377-86.

8. Vokes EE, Liebowitz DN, Weichselbaum RR. Nasopharyngeal carcinoma. Lancet 1997; 350: 1087-91.

9. Wang X, Li L, Hu C, et al. Patterns of level II node metastasis in nasopharyngeal carcinoma. Radiother Oncol 2008; 89: 2832.

10. Bartel DP. MicroRNAs: genomics, biogenesis, mechanism, and function. Cell 2004; 116: 281-97.

11. Liu N, Chen NY, Ciu RX, et al. Prognostic value of a microRNA signature in nasopharyngeal carcinoma: a microRNA expression analysis. Lancet Oncol 2012; 13: 633-41.

12. Fujishima A, Honda K. Electrochemical photolysis of water at a semiconductor electrode. Nature 1972; 238: 37-8.

13. Tallósy SP, Janovák L, Ménesi J, et al. Investigation of the antibacterial effects of silver-modified $\mathrm{TiO}_{2}$ and $\mathrm{ZnO}$ plasmonic photocatalysts embedded in polymer thin films. Environ Sci Pollut Res Int 2014; 21: 11155-67.

14. Veres Á, Rica T, Janovák L, et al. Silver and gold modified plasmonic $\mathrm{TiO}_{2}$ hybrid films for photocatalytic decomposition of ethanol under visible light. Catal Today 2012; 181: 156-62.

15. Marton A, Vizler C, Kusz E, et al. Melanoma cell-derived exosomes alter macrophage and dendritic cell functions in vitro. Immunol Lett 2012; 148: 34-8.

16. Hutter JL, Bechhoefer J. Calibration of atomic-force microscope tips. Rev Sci Instrum 1993; 64: 1868-73.

17. Sader JE, Chon JWM, Mulvaney P. Calibration of rectangular atomic force microscope cantilevers. Rev Sci Instrum 1999; 70: $3967-9$.

18. Higgins MJ, Proksch R, Sader JE, et al. Noninvasive determination of optical lever sensitivity in atomic force microscopy. Rev Sci Instrum 2006; 77: 013701. 
19. Sader JE, Sanelli JA, Adamson BD, et al. Spring constant calibration of atomic force microscope cantilevers of arbitrary shape. Rev Sci Instrum 2012; 83: 103705.

20. Ebner A, Hinterdorfer P, Gruber HJ. Comparison of different aminofunctionalization strategies for attachment of single antibodies to AFM cantilevers. Ultramicroscopy 2007; 107: 922-7.

21. Aekbote BL, Jacak J, Schütz GJ, et al. Aminosilane-based functionalization of two-photon polymerized 3D SU-8 microstructures. Eur Polym J 2012; 48: 1745-54.

22. Witwer KW, Buzas EI, Bemis LT, et al. Standardization of sample collection, isolation and analysis methods in extracellular vesicle research. J Extracell Vesicles 2013; $27: 2$.

23. Wirth R, Kovács E, Maróti G, Bagi Z, Rákhely G, Kovács KL. Characterization of a biogas-producing microbial community by short-read next generation DNA sequencing. Biotechnol Biofuels 2012; 5: 41.

24. Zhou Y, Huang Z, Wu S, et al. miR-33a is up-regulated in chemoresistant osteosarcoma and promotes osteosarcoma cell resistance to cisplatin by down-regulating TWIST. J Exp Clin Cancer Res 2014; 33: 12.

25. Yáñez-Mó M, Siljander PR, Andreu Z, et al. Biological properties of extracellular vesicles and their physiological functions. J Extracell Vesicles 2015; 4: 27066.

26. Hortobagyi GN. Anthracyclines in the treatment of cancer. An overview. Drugs 1997; 54(Suppl 4): 1-7.

27. Pang B, Qiao X, Janssen L, et al. Drug-induced histone eviction from open chromatin contributes to the chemotherapeutic effects of doxorubicin. Nat Commun 2013; 4: 1908.

28. Filomeni G, De Zio D, Cecconi F. Oxidative stress and autophagy: the clash between damage and metabolic needs. Cell Death Differ 2015; 22: 377-88.

29. King HW, Michael MZ, Gleadle JM. Hypoxic enhancement of exosome release by breast cancer cells. BMC Cancer 2012; 12: 421.

30. Eldh M, Ekström K, Valadi H, et al. Exosomes communicate protective messages during oxidative stress; possible role of exosomal shuttle RNA. PLoS One 2010; 5: e15353.
31. Hedlund M, Nagaeva O, Kargl D, Baranov V, MinchevaNilsson L. Thermal- and oxidative stress causes enhanced release of NKG2D ligand-bearing immunosuppressive exosomes in leukemia/lymphoma T and B cells. PLoS One 2011; 6: e16899.

32. Lv LH, Wan YL, Lin Y, et al. Anticancer drugs cause release of exosomes with heat shock proteins from human hepatocellular carcinoma cells that elicit effective natural killer cell antitumor responses in vitro. J Biol Chem 2012; 287: $15874-85$.

33. Kucharzewska P, Belting M. Emerging roles of extracellular vesicles in the adaptive response of tumour cells to microenvironmental stress. J Extracell Vesicles 2013; 5: 2.

34. Spano D, Zollo M. Tumor microenvironment: a main actor in the metastasis process. Clin Exp Metastasis 2012; 29: 381-95.

35. Quail DF, Joyce JA. Microenvironmental regulation of tumor progression and metastasis. Nat Med 2013; 19: 1423-37.

\section{Acknowledgements}

This research was funded by OTKA PD 84064, OTKA K 112493, TAMOP4.2.2-A-11/1/KONV-2012-0025, and IPA HUSRB/1203/214/230. The authors would like to thank Gábor Braunitzer and János Minarovits for their helpful comments.

\section{Conflict of interest}

The authors declare no conflict of interest.

\section{Supporting Information}

Additional Supporting Information may be found in the online version of this article:

Data S1 References. 


\section{Supporting information}

\section{References of Table 1}

1. Nie G, Duan H, Li X, et al. MicroRNA-205 promotes the tumorigenesis of nasopharyngeal carcinoma through targeting tumor protein p53-inducible nuclear protein 1. Mol Med Rep 2015; 12(4): 5715-22.

2. Qu C, Liang Z, Huang J, et al. MiR-205 determines the radioresistance of human nasopharyngeal carcinoma by directlytargeting PTEN. Cell Cycle 2012; 11(4): 785-96.

3. Wong TS, Man OY, Tsang CM, et al. MicroRNA let-7 suppresses nasopharyngeal carcinoma cells proliferation throughdownregulating c-Myc expression. J Cancer Res Clin Oncol 2011; 137(3): 415-22.

4. Ou H, Li Y, Kang M. Activation of miR-21 by STAT3 induces proliferation and suppresses apoptosis in nasopharyngeal carcinoma by targeting PTEN gene. PLoS One 2014; 9(11): e109929.

5. Li Y, Yan L, Zhang W, et al. miR-21 inhibitor suppresses proliferation and migration of nasopharyngeal carcinoma cells through down-regulation of BCL2 expression. Int $\mathrm{J}$ Clin Exp Pathol 2014; 7(6): 3478-87.

6. Yang GD, Huang TJ, Peng LX, et al. Epstein-Barr Virus_Encoded LMP1 upregulates microRNA-21 to promote the resistance ofnasopharyngeal carcinoma cells to cisplatin-induced Apoptosis by suppressing PDCD4 and Fas-L. PLoS One 2013; 8(10): e 78355.

7. Wu Z-s, Wu Q, Wang C-q, et al. MiR-339-5p inhibits breast cancer cell migration and invasion in vitro and may be a potential biomarker for breast cancer prognosis. BMC Cancer 2010; 10: 542.

8. Zhou C, Liu G, Wang L, et al. MiR-339-5p regulates the growth, colony formation and metastasis of colorectal cancer cells by targeting PRL-1. PLoS One 2013; 8(5): e63142.

9. Li Y, Zhao W, Bao P, et al. miR-339-5p inhibits cell migration and invasion in vitro and may be associated with the tumor-node-metastasis staging and lymph node metastasis of non-small cell lung cancer. Oncol Lett 2014; 8(2): 719-725.

10. Süslüer SY, Biray Avci Ç, Şiğva ZÖD, et al. Detection of miRNA Expression Alteration in Diffuse and High Grade Glial Tumors. J Neurol Sci 2015; 32(1), 64-88.

11. Khan FH, Pandian V, Ramraj S, Aravindan S, Herman TS, Aravindan N. Reorganization of metastamiRs in the evolution of metastatic aggressive neuroblastoma cells. BMC Genomics 2015; 16(1): 501.

12. Xu HS, Zong HL, Shang M, et al. MiR-324-5p inhibits proliferation of glioma by target regulation of GLI1. Eur Rev Med Pharmacol Sci 2014; 18(6): 828-32. 
13. Lyu X, Fang W, Cai L, et al. TGF $\beta$ R2 is a major target of miR-93 in nasopharyngeal carcinoma aggressiveness. Mol Cancer 2014; 13: 51.

14. Hirata H, Hinoda Y, Shahryari V, et al. Genistein downregulates onco-miR-1260b and upregulates sFRP1 and Smad4 via demethylation and histone modification in prostate cancer cells. Br J Cancer 2014; 110(6): 1645-54.

15. Liao W, Gu C, Huang A, Yao J, Sun R. MicroRNA-33b inhibits tumor cell growth and is associated with prognosis in colorectal cancer patients. Clin Transl Oncol (in press).

16. $\mathrm{Xu} \mathrm{N}, \mathrm{Li} \mathrm{Z}, \mathrm{Yu} \mathrm{Z}$, et al. MicroRNA-33b suppresses migration and invasion by targeting c-Myc in osteosarcoma cells. PLoS One 2014; 9(12): e115300.

17. Lin J, Huang S, Wu S, et al. MicroRNA-423 promotes cell growth and regulates $\mathrm{G}(1) / \mathrm{S}$ transition by targeting p21Cip1/Waf1 in hepatocellular carcinoma. Carcinogenesis 2011; 32(11): 1641-7.

18. Kitago M, Martinez SR, Nakamura T, Sim MS, Hoon DS. Regulation of RUNX3 tumor suppressor gene expression in cutaneous melanoma. Clin Cancer Res 2009; 15(9): 2988-94.

19. Yang F, Nam S, Brown CE, et al. A novel berbamine derivative inhibits cell viability and induces apoptosis in cancer stem-like cells of human glioblastoma, via upregulation of miRNA-4284 and JNK/AP-1 signaling. PLoS One 2014; 9(4): e94443.

20. Chen Z, Jin Y, Yu D, et al. Down-regulation of the microRNA-99 family members in head and neck squamous cell carcinoma. Oral Oncol 2012; 48(8): 686-91.

21. Kang J, Lee S, Lee $S$, et al. microRNA-99b acts as a tumor suppressor in non-small cell lung cancer by directly targeting fibroblast growth factor receptor 3. Exp Ther Med 2012; 3(1): 149-153.

22. Sun D, Lee YS, Malhotra A, et al. miR-99 family of MicroRNAs suppresses the expression of prostate-specific antigen and prostate cancer cell proliferation. Cancer Res 2011; 71(4): 1313-24.

23. Tang R, Qi Q, Wu R, et al. The polymorphic terminal-loop of pre-miR-1307 binding with MBNL1 contributes to colorectal carcinogenesis via interference with Dicer1 recruitment. Carcinogenesis. 2015; 36(8): 867-75.

24. Liu N, Jiang N, Guo R, et al. MiR-451 inhibits cell growth and invasion by targeting MIF and is associated with survival in nasopharyngeal carcinoma. Mol Cancer 2013; 12(1): 123 .

25. Fukumoto I, Kinoshita T, Hanazawa T, et al. Identification of tumour suppressive microRNA-451a in hypopharyngeal squamous cell carcinoma based on microRNA expression signature. Br J Cancer 2014; 111(2): 386-94. 
26. Kuo PL, Liao SH, Hung JY, Huang MS, Hsu YL. MicroRNA-33a functions as a bone metastasis suppressor in lung cancer by targeting parathyroid hormone related protein. Biochim Biophys Acta 2013; 1830(6): 3756-66.

27. Zhu J, Chen L, Zou L et al. MiR-20b, -21, and -130b inhibit PTEN expression resulting in B7-H1 over-expression in advanced colorectal cancer. Hum Immunol 2014; 75(4): 348-53.

28. Lai KW, Koh KX, Loh M, et al. MicroRNA-130b regulates the tumour suppressor RUNX3 in gastric cancer. Eur J Cancer 2010; 46(8): 1456-63.

29. Zhao G, Zhang J-g, Shi Y, et al. MiR-130b Is a Prognostic Marker and Inhibits Cell Proliferation and Invasion in Pancreatic Cancer through Targeting STAT3. PLoS One 2013; 8(9): e73803.

30. Zhang C, Fang X, Li W, et al. Influence of recombinant lentiviral vector encoding miR-15a/16-1 in biological features of human nasopharyngeal carcinoma CNE-2Z cells. Cancer Biother Radiopharm 2014; 29(10): 422-7.

31. Zhu JY, Pfuhl T, Motsch N, et al. Identification of novel Epstein-Barr virus microRNA genes from nasopharyngeal carcinomas. J Virol 2009; 83(7): 3333-41.

32. Yao K, He L, Gan Y, Zeng Q, Dai Y, Tan J. MiR-186 suppresses the growth and metastasis of bladder cancer by targeting NSBP1. Diagn Pathol 2015; 10: 146.

33. Cui G, Cui M, Li Y, et al. MiR-186 targets ROCK1 to suppress the growth and metastasis of NSCLC cells. Tumour Biol 2014; 35(9): 8933-7.

34. Ji Y, He Y, Liu L, Chong X. MiRNA-26b regulates the expression of cyclooxygenase2 in desferrioxamine-treated CNE cells. FEBS Lett 2010; 584(5): 961-7.

35. Cui R, Kim T, Fassan M, et al. MicroRNA-224 is implicated in lung cancer pathogenesis through targeting caspase-3 and caspase-7. Oncotarget 2015; 6(26): 21802-15.

36. He X, Zhang Z, Li M, et al. Expression and role of oncogenic miRNA-224 in esophageal squamous cell carcinoma. BMC Cancer 2015; 15(1): 575.

37. Cheung CC, Chung GT, Lun SW, et al. miR-31 is consistently inactivated in EBVassociated nasopharyngeal carcinoma andcontributes to its tumorigenesis. Mol Cancer 2014; 13: 184.

38. Lou F, Ma HN, Xu L Chen M, Zhu YB. Two polymorphisms of CD44 3'UTR weaken the binding of miRNAs and associate with naso-pharyngeal carcinoma in a Chinese population. Eur Rev Med Pharmacol Sci 2014;18(17):2444-52. 